\title{
HYDROGEOLOGIC DATA FOR THE NORTHERN ROCKY MOUNTAINS INTERMONTANE BASINS, MONTANA
}

by DeAnn M. Dutton, Sean M. Lawlor, David W. Briar, and Ruth E. Tresch

\section{U.S. GEOLOGICAL SURVEY}

Open-File Report 95-143

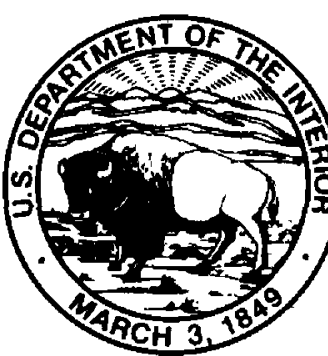




\title{
U.S. DEPARTMENT OF THE INTERIOR BRUCE BABBITT, Secretary
}

\author{
U.S. GEOLOGICAL SURVEY \\ Gordon P. Eaton, Director
}

For additional information write to:

Copies of this report may be purchased from:

District Chief

U.S. Geological Survey

428 Federal Building

Drawer 10076

301 South Park

Helena, MT 59626-0076
U.S. Geological Survey

Earth Science Information Center

Open-File Reports Section

Box 25286, MS 517

Denver Federal Center

Denver, CO 80225-0286 


\section{CONTENTS}

Abstract

Introduction

Well-numbering system

Reference

Data

\section{ILLUSTRATIONS}

Plate 1. Map showing location of selected wells, Northern Rocky Mountains intermontane basins, Montana In pocket

Figure 1. Diagram showing well-numbering system.

2. Hydrographs for selected wells, Northern Rocky Mountains intermontane basins, Montana

\section{TABLE}

Table 1. Records of selected wells by basin, Northern Rocky Mountains intermontane basins, Montana

\section{CONVERSION FACTORS AND VERTICAL DATUM}

\begin{tabular}{rll}
\hline Multiply inch-pound unit & By & To obtaln S1 unit \\
acre & 0.4047 & bectare \\
fool $(\mathrm{ft})$ & 0.3048 & meter \\
gallon per minute $(\mathrm{gal} / \mathrm{min})$ & 0.06309 & liter per second \\
inch $(\mathrm{in.})$ & 0.2070 & liter per second per meter \\
gallon per minute per fool [(gal/min)/ft] & 25.4 & millimeter \\
square mile $\left(\mathrm{mi}^{2}\right)$ & 2.59 & square kilometer \\
\hline
\end{tabular}

Temperature in degrees Celsius $\left({ }^{\circ} \mathrm{C}\right)$ can be converted to degrees Fahrenheit $\left({ }^{\circ} \mathrm{F}\right)$ by the equation:

$$
{ }^{\circ} \mathrm{F}=9 / 5\left({ }^{\circ} \mathrm{C}\right)+32
$$

Sea level: In this report "sea level" refers to the National Geodetic Vertical Datum of 1929 ( NGVD of 1929)--a geodetic datum derived from a general adjustment of the first-order level nets of both the United States and Canada, formerly called Sea Level Datum of 1929. 


\title{
HYDROGEOLOGIC DATA FOR THE NORTHERN ROCKY MOUNTAINS INTERMONTANE BASINS, MONTANA
}

\author{
by DeAnn M. Dutton, Sean M. Lawlor, David W. Briar, and Ruth E. Tresch
}

\section{Abstract}

The U.S. Geological Survey began a Regional Aquifer-System Analysis of the Northern Rocky Mountains intermontane basins of western Montana and central and northern Idaho in 1990 to establish a regional framework of information for aquifers in 54 intermontane basins in an area of about 77,500 square miles. Selected hydrogeologic data have been used as part of this analysis to define the hydrologic systems. Records of 1,376 wells completed in 31 of the 34 intermontane basins in the Montana part of the study area are tabulated in this report. Data were not collected in the North Fork Flathead River Valley, Silver Bow Creek Valley, or upper Madison River Valley. Data consist of location (well number), altitude of land surface, date well constructed, geologic unit, depth of well, diameter of casing, type of finish, top of open interval, primary use of water, water level, date water level measured, discharge, specific capacity, source of discharge data, type of $\log$ available, date water-quality parameters measured, specific conductance, $\mathrm{pH}$, and temperature. Hydrographs for selected wells also are included. Locations of wells and basins are shown on the accompanying plate.

\section{INTRODUCTION}

Numerous intermontane basins in western Montana contain consolidated to unconsolidated basin-fill deposits of Tertiary and Quaternary age that commonly are saturated with water. Ground-water flow in each basin is controlled primarily by the distribution and nature of deposits that compose the basin-fill aquifers. These deposits were derived from the rocks surrounding the basins; the geologic processes that created the basins controlled deposition of the fill material. In many areas, these deposits are the only source of water of adequate quantity and suitable quality for publicsupply, domestic, stock, irrigation, commercial, and industrial use. Regional and local concerns about ground-water availability and quality are numerous (Clark and Kendy, 1992). Population growth and continued development in western Montana will increase the need for water, particularly ground water. However, little information is available about this valuable resource on either a regional or local scale.

The U.S. Geological Survey began an investigation in 1990 of the ground-water resources of the Northern Rocky Mountains intermontane basins in western Montana and central and northern Idaho as part of the Regional Aquifer-System Analysis (RASA) program. The purpose of the investigation is to determine the aquifer boundaries, the quantity and quality of the water within the aquifers, and recharge characteristics of the aquifer systems. The analysis establishes a regional framework of information for aquifers in 54 intermontane basins in an area of about 77,500 square miles.

The purpose of this report is to summarize the hydrogeologic data that were collected and compiled for the Montana part of the study. Specifically, this report presents records of 1,376 wells completed in 31 of 34 intermontane basins in the Montana part of the study area. Data were not collected in the North Fork Flathead River Valley, Silver Bow Creek Valley, or upper Madison River Valley. Some of the wells inventoried were constructed as early as the late 1800 's. The majority of the wells are completed in unconsolidated deposits of Tertiary and Quaternary age. Some of these data have been published in previous reports but are included here to provide all the data in a single report. The majority of the data were collected in 1991. Wells are listed in table 1 . Well and basin locations are shown on plate 1 . Hydrographs for 32 wells for which 7 or more years of water-level data are available are shown in figure 2. Dashed lines between data points do not infer actual water-level trends.

\section{WELL-NUMBERING SYSTEM}

In this report, wells are assigned a number according to geographic position within the rectangular grid system used for the subdivision of public lands 
(fig. 1). The well number consists of as many as 14 characters. The first three characters specify the township and its position north (N) or south (S) of the Montana Base Line. The next three characters specify the range and its position west $(W)$ or east $(E)$ of the Montana Principal Meridian. The next two characters are the section number. The next one to four characters designate the quarter section (160-acre tract), quarterquarter section (40-acre tract), quarter-quarter-quarter section (10-acre tract), and quarter-quarter-quarterquarter section (2 1/2-acre tract), respectively, in which the well is located. The subdivisions of the section are designated $A, B, C$, and $D$ in a counterclockwise direc- tion beginning in the northeast quadrant. The last two characters are a sequence number indicating the order in which the well was inventoried within the described tract. For example, as shown on figure 1, well 11N03W21DDAD01 is the first well inventoried in the SE 1/4 NE 1/4 SE 1/4 SE1/4 sec. 21, T. 11 N., R. 3 W.

\section{REFERENCE}

Clark, D.W., and Kendy, Eloise, 1992, Plan of study for the Regional Aquifer-System Analysis of the Northern Rocky Mountains Intermontane Basins, Montana and Idaho: U.S. Geological Survey Water-Resources Investigations Report 92-4116, 16 p.

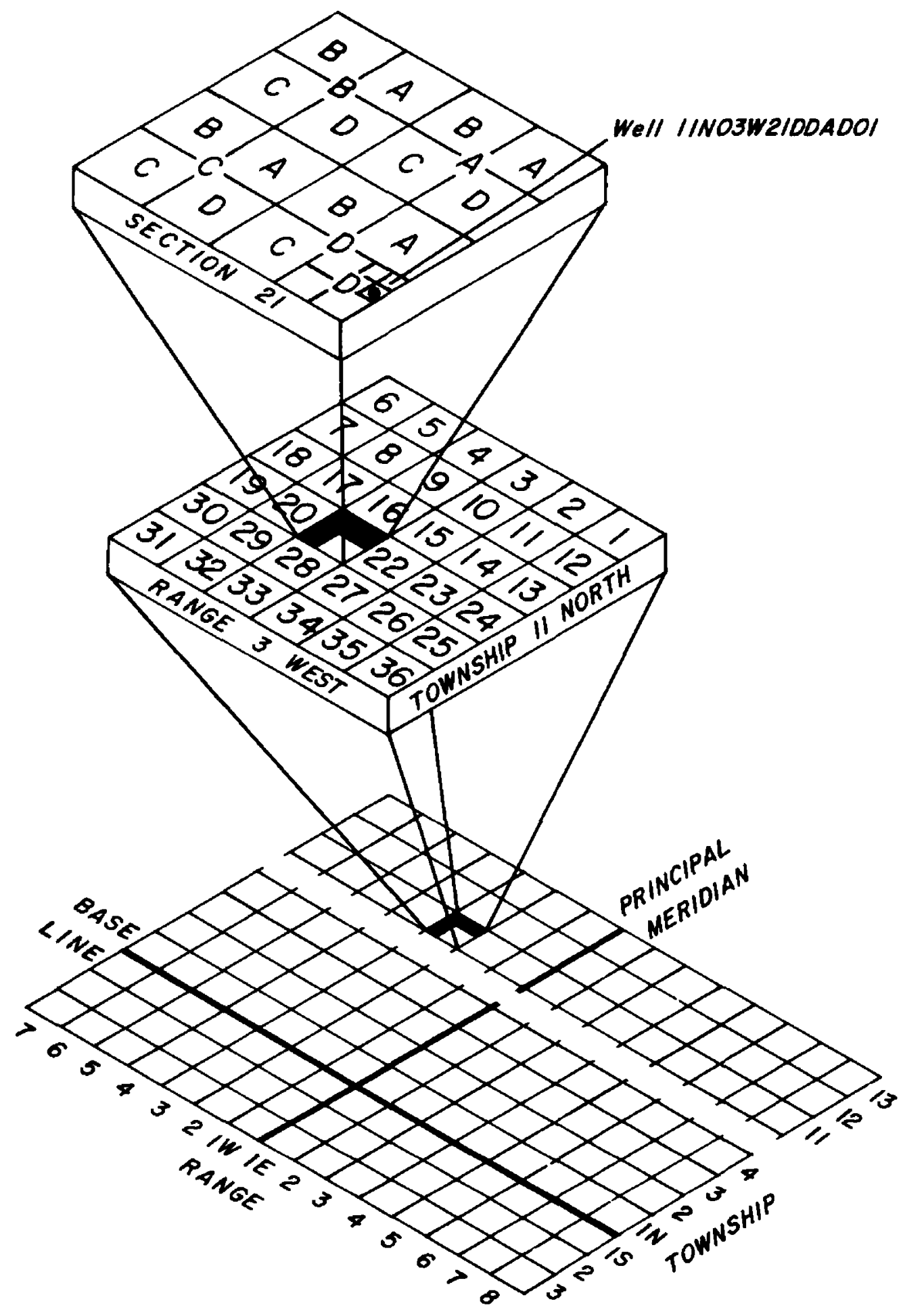

Figure 1. Well-numbering system. 
DATA

Data

3 
Table 1. Records of selected wells by basin, Northern Rocky Mountains intermontane basins, Montana

Well number -- numbering system described in text.

Altitude of land surface -- in feet above sea level.

Geologic unit - Qg. Quatemary glacial deposits; Qal, Quatemary alluvium; QTsu, Quatemary-Tertiary sediments, undifferentiated; QTKe, Quaternary-Tertiary-Cretaceous extrusive igneous rocks; Ts, Tertiary sediments; TKi, Tertiary-Cretaceous intrusive igneous rocks; Mzsh, Mesozoic rocks (predominantly shale); Pzls, Paleozoic rocks (predominantly limestone); Yms, Precambrian (Middle Proterozoic) metasedimentary rocks; KAm, CretaceousPrecambrian (Archean) metamorphic rocks; Unk, unknown.

Depth of well -- in feet below land surface.

Diameter of casing --in inches.

Type of finish -- F, perforations with gravel; $G$, screen with gravel; $H$, horizontal gallery; $O$, open end; $P$, perforated or slotted; $S$, screen; $T$, sand point; $X$, open hole; $Z$, other.

Top of open interval -- in feet below land surface.

Primary use of water -- C, commercial; D, dewater, F, fire; H, domestic; I, irigation; $K$, mining; $N$, industrial; $P$, public supply; $R$, recreation; $S$, stock; $T$. institution; $\mathrm{U}$, unused.

Water level - in feet below or above $(+)$ land surface; *, approximate water level; flowing, water level above land surface.

Discharge -- in gallons per minute (gal/min), reported to two significant figures when value is greater than $10 \mathrm{gal} / \mathrm{min}$ or to one significant figure when value is less than $10 \mathrm{gal} / \mathrm{min}$.

Specific capacity -- in gallons per minute per foot [(gal/min)/ft] of drawdown in a well, reported to two significant figures; values less than one are reported to the nearest tenth.

Source of discharge data-- A, other govemunent agency; D, driller, L, logs; O, owner, R, other reported; S, USGS.

Type of log available -- D, driller.

Specific conductance -- in microsiemens per centimeter at 25 degrees Celsius; onsite measurement.

pH -- in standard units; onsite measurement.

Temperature -- in degrees Celsius; onsite measurement.

Abbreviations -- ${ }^{\circ} \mathrm{C}$, degrees Celsius; $\mathrm{ft}$, feet; gal/min, gallons per minute; [(gal/min)/ft], gallons per minute per foot; in., inch; $\mu \mathrm{S} / \mathrm{cm}, \mathrm{microsiemens} \mathrm{per}$ centimeter at $25^{\circ} \mathrm{C}$.

Symbols: <, less than; - or --, no data. 
Table 1. Records of selected wells by basin, Northern Rocky Mountains intermontane basins, Montana (Continued)

\begin{tabular}{|c|c|c|c|c|c|c|c|c|c|}
\hline Well number & $\begin{array}{l}\text { Altitude } \\
\text { of land } \\
\text { surface } \\
\text { (fit) }\end{array}$ & $\begin{array}{l}\text { Date well } \\
\text { constructed }\end{array}$ & $\begin{array}{c}\text { Geologic } \\
\text { unit }\end{array}$ & $\begin{array}{c}\text { Depth } \\
\text { of } \\
\text { well } \\
\text { (ft) }\end{array}$ & $\begin{array}{l}\text { Dlam- } \\
\text { otor } \\
\text { of } \\
\text { casing } \\
\text { (In.) }\end{array}$ & $\begin{array}{c}\text { Type } \\
\text { of } \\
\text { finish }\end{array}$ & $\begin{array}{l}\text { Top of } \\
\text { open } \\
\text { Inter- } \\
\text { val } \\
\text { (ft) }\end{array}$ & $\begin{array}{c}\text { Primary } \\
\text { use of } \\
\text { water }\end{array}$ & $\begin{array}{c}\text { Date water } \\
\text { lovel } \\
\text { measured }\end{array}$ \\
\hline \multicolumn{10}{|c|}{ Avon Valley } \\
\hline 11N08W20ACAC01 & 5,325 & $09-26-57$ & Qal & 82 & 6.3 & - & -- & $\mathbf{H}$ & $04-10-91$ \\
\hline 11 N08W34BDCC01 & 5,185 & $12-16-87$ & Qal & 57 & - & $\mathbf{P}$ & 49 & $\mathbf{H}$ & $04-10-91$ \\
\hline 10N09W13BBCC01 & 5,210 & 05-28-89 & Mzsh & 200 & 6.3 & $\mathbf{P}$ & 80 & $\mathbf{H}$ & $04-10-91$ \\
\hline 10N08W04ADDA01 & 5,035 & $01-03-73$ & Qal & 52 & 6.0 & $\mathbf{P}$ & 45 & $\mathbf{H}$ & $04-10-91$ \\
\hline 10N08W04ADDB01 & 5,036 & -- & QTsu & 29 & -- & - & -- & $\mathbf{S}$ & $04-10-91$ \\
\hline 10N08W06AADD01 & 4,925 & $-\quad-74$ & QTsu & 75 & 6.0 & - & - & $\mathbf{H}$ & 04-09-91 \\
\hline I0N08W08DDBC01 & 4,808 & $07-22-86$ & Ts & 124 & 6.6 & $\mathbf{P}$ & 80 & $\mathbf{H}$ & 04-09-91 \\
\hline 10N08W08DDCB01 & 4,810 & $-\quad-73$ & Ts & 100 & 6.0 & - & -- & $\mathbf{H}$ & $07-21-76$ \\
\hline 10N08W21ABDB01 & 4,740 & $09-20-60$ & Ts & 200 & 6.0 & $\mathbf{P}$ & 30 & $\mathbf{U}$ & $04-09-91$ \\
\hline 10N08W27BAAB01 & 4,715 & -- & Qal & 31 & 6.0 & - &.- & $\mathbf{T}$ & 04-09-91 \\
\hline 10N08W27BABA0I & 4,710 & 08-08-89 & Qal & 40 & 6.6 & $\mathbf{o}$ & -- & $\mathbf{H}$ & $04-09-91$ \\
\hline 10N08W27BABA02 & 4,710 & $10-28-76$ & Qal & 160 & 6.0 & $\mathbf{P}$ & 55 & $\mathbf{H}$ & $04-09-91$ \\
\hline 10N07W17ADCD01 & 4,950 & -- & Qal & 14 & 6.0 & - & -- & $\mathbf{H}$ & 04-04-91 \\
\hline 10N07W 17DABB01 & 4,945 & $10-07-82$ & Ts & 65 & 6.6 & 0 & - & $\mathbf{U}$ & $04-04-91$ \\
\hline 10NO7W30BBC 01 & 4,825 & $-\quad-61$ & Ts & 70 & 6.0 & o & $\cdots$ & $\mathbf{U}$ & $04-04-91$ \\
\hline 10N07W31CAAC01 & 4,865 & $06-22-77$ & QTKe & 67 & 6.0 & o & - & $\mathbf{P}$ & $04-09-91$ \\
\hline 09N07W02ADDB01 & 5,040 & $03-01-88$ & Qal & 55 & 6.6 & 0 &. & $\mathbf{P}$ & 04-04-91 \\
\hline 09N07W02DABA01 & 5,030 & $09-30-88$ & Qal & 120 & 6.6 & $\mathbf{Z}$ & 30 & $\mathbf{K}$ & $04-04-91$ \\
\hline 09N06W05ACCD01 & 5,260 & $02-10-74$ & QTKe & 57 & 6.0 & $\mathbf{P}$ & 45 & $\mathbf{H}$ & $02-17-74$ \\
\hline 09N06W06DCDA01 & 5,160 & -- & QTsu & 68 & -- & - & -- & $\mathbf{H}$ & $04-04-91$ \\
\hline 09N06W 18BAAC01 & 5,190 & -- & Qal & 36 & -- & - & -- & $\mathbf{H}$ & $04-04-91$ \\
\hline 09N06W18BBBA0I & 5,250 & $07-28-86$ & QTsu & 163 & 6.0 & $\mathbf{Z}$ & 83 & $\mathbf{U}$ & $04-04-91$ \\
\hline 09N06W 19AABC01 & 5,240 & 06-05-79 & Qal & 46 & 6.0 & o & -- & $\mathbf{H}$ & $04-04-91$ \\
\hline 09N06W30CABA01 & 5,315 & $06-26-81$ & Qal & 61 & 6.6 & $\mathrm{o}$ & -- & $\mathbf{H}$ & 04-04-91 \\
\hline \multicolumn{10}{|c|}{ Beaverhead Valley } \\
\hline 03S06W35BDAB01 & 4,718 & $01-13-90$ & Ts & 66 & 6.0 & o & -- & $\mathbf{H}$ & 09-18-91 \\
\hline 04S09W25AAAA01 & 4,970 & $09-11-80$ & QTsu & 48 & 6.0 & $\mathbf{P}$ & 41 & $\mathbf{H}$ & $06-21-91$ \\
\hline 04S07W28BDA $\Lambda 01$ & 4,784 & -- & QTsu & 31 & 6.0 & - & - & $\mathrm{U}$ & $06-27-91$ \\
\hline 04S07W28BDDC01 & 4,784 & -- & QTsu & 30 & 6.0 & - & - & $\mathbf{U}$ & $06-27-91$ \\
\hline 04S07W28BDDC02 & 4,784 & -- & Qal & 19 & 6.0 & - & -- & $\mathrm{U}$ & $06-27-91$ \\
\hline 04SO7W28CDCA01 & 4,795 & $09-28-86$ & Qal & 30 & 6.0 & $\mathbf{P}$ & 20 & $\mathbf{H}$ & $06-27-91$ \\
\hline 04S07W32AABA01 & 4,802 & -- & Qal & 25 & 6.0 & - & - & $\mathbf{H}$ & $06-27-91$ \\
\hline 04S07W32CBCB01 & 4,815 & -- & QTsu & 36 & 6.0 & - & -- & $\mathbf{H}$ & $06-27-91$ \\
\hline 04S07W33BDBA01 & 4,890 & $05-12-86$ & QTsu & 165 & 6.0 & $\mathbf{P}$ & 155 & $\mathbf{S}$ & $06-27-91$ \\
\hline 04S06W16AAAA02 & 4,720 & $11--64$ & Ts & 58 & 1.3 & $\mathbf{T}$ & 3 & $\mathbf{U}$ & $09-19-91$ \\
\hline
\end{tabular}


Table 1. Records of selected wells by basin, Northern Rocky Mountains intermontane basins, Montana (Continued)

\begin{tabular}{|c|c|c|c|c|c|c|c|c|c|}
\hline $\begin{array}{c}\text { Water loval } \\
\text { (ft) }\end{array}$ & $\begin{array}{c}\text { Dle- } \\
\text { charge } \\
\text { (gal/ } \\
\text { min) }\end{array}$ & $\begin{array}{c}\text { Speclfic } \\
\text { capaclty } \\
\text { [(gal } \\
\text { min)/ft] }\end{array}$ & $\begin{array}{l}\text { Source } \\
\text { of dla- } \\
\text { charge } \\
\text { date }\end{array}$ & $\begin{array}{l}\text { Typo } \\
\text { of log } \\
\text { avall- } \\
\text { able }\end{array}$ & $\begin{array}{c}\text { Date } \\
\text { water- } \\
\text { quallty } \\
\text { paramater } \\
\text { measured }\end{array}$ & $\begin{array}{l}\text { Specific } \\
\text { conduc- } \\
\text { tence } \\
(\mu \mathrm{s} / \mathrm{cm})\end{array}$ & $\begin{array}{c}\text { pH } \\
\text { (stan- } \\
\text { dard } \\
\text { units) }\end{array}$ & $\begin{array}{l}\text { Tem- } \\
\text { per- } \\
\text { ature } \\
\left({ }^{\circ} \mathrm{C}\right)\end{array}$ & Well number \\
\hline \multicolumn{10}{|c|}{ Avon Valley } \\
\hline 66.61 & -- & -- & - & - & $04-10-91$ & 331 & 8.0 & 4.0 & 11 NO8W20ACAC01 \\
\hline 33.50 & 40 & 10 & D & D & $04-10-91$ & 428 & 7.4 & 7.5 & $11 \mathrm{N08W34BDCC01}$ \\
\hline 4.86 & 5 & -- & D & D & $04-10-91$ & 313 & 8.0 & 3.5 & $10 \mathrm{~N} 09 \mathrm{~W} 13 \mathrm{BBCC} 01$ \\
\hline 10.84 & 15 & .6 & D & D & $04-10-91$ & 401 & 7.2 & 7.5 & 10N08W04ADDA01 \\
\hline 10.83 & -- & -. & - & - & -- & -- & - & -- & I0N08W04ADDB01 \\
\hline 12.10 & -- & -- & - & - & -- & -- & -- & .. & 10N08W06AADD01 \\
\hline 35.99 & 8 & -. & D & D & 04-09-91 & 1,090 & 8.2 & 6.5 & 10N08W08DDBC01 \\
\hline 27.60 & -- & -- & - & - & 04-09-91 & 554 & 8.4 & 9.5 & 10N08W08DDCB01 \\
\hline 17.18 & 20 & .9 & D & D & - & -- & - & -- & 10N08W21ABDB01 \\
\hline 22.70 & -- & -- & - & - & -- & -- & -- & -- & 10N08W27BAAB01 \\
\hline 20.32 & 15 & -- & D & D & $04-09-91$ & 227 & 7.2 & 7.5 & 10N08W27BABA0I \\
\hline 20.85 & 3 & -- & D & D & -- & -- & -- & -- & 10N08W27BABA02 \\
\hline 2.07 & -- & -- & - & - & 04-04-91 & 453 & 7.3 & -- & 10N07W17ADCD01 \\
\hline 14.48 & 20 & .4 & D & D & - & -- & -- & -- & 10N07W $17 \mathrm{DABB} 01$ \\
\hline 34.95 & -- & -- & - & - & -- & - & -- & -- & $10 N 07 W 30 B B C 01$ \\
\hline 18.92 & 20 & .5 & D & D & - & -- & -- & -. & 10N07W31CAAC01 \\
\hline 34.23 & -- & -- & - & D & $04-04-91$ & 306 & 7.5 & 9.5 & 09N07W02ADDB01 \\
\hline 26.40 & 10 & .1 & D & D & -- & -- & -- & -- & 09N07W02DABA01 \\
\hline 24.96 & 7 & .2 & D & D & 08-13-92 & 285 & 7.0 & 7.5 & 09N06W05ACCD01 \\
\hline 24.20 & -- & -- & - & - & $04-04-91$ & 218 & 7.4 & 8.0 & 09N06W06DCDA01 \\
\hline 23.63 & -- & -- & - & - & 04-04-91 & 226 & 6.6 & 6.5 & 09N06W18BAAC01 \\
\hline 48.22 & 20 & .2 & D & D & -- & -- & -. & -- & 09N06W 18BBBA01 \\
\hline 20.64 & 15 & .9 & D & D & -- & -- & -- & -- & 09N06W19AABC01 \\
\hline 22.39 & 15 & -- & D & D & - & -- & -- & - & 09N06W30CABA01 \\
\hline
\end{tabular}

\section{Beaverhead Valley}

\begin{tabular}{|c|c|c|c|c|c|c|c|c|c|}
\hline 44.13 & 12 & .. & D & D & 09-18-91 & 700 & -- & 9.5 & 03S06W35BDAB01 \\
\hline 30.38 & 15 & -- & D & D & $06-21-91$ & 207 & 7.2 & 11.0 & 04S09W25AAAA01 \\
\hline Flowing & .- & -- & - & - & $06-27-91$ & 2,270 & -- & 51.5 & 04S07W28BDAA01 \\
\hline 2.50 & -. & -- & - & - & -- & -- & - & -- & 04S07W28BDDC01 \\
\hline 3.06 & -- & -- & - & - & -- & -- & -- & -- & 04S07W28BDDC02 \\
\hline 4.50 & 20 & 3.3 & D & D & -. & -. & -- & -- & 04S07W28CDCA01 \\
\hline 3.80 & -- & -- & - & - & -- & -- & -- & -- & 04507W32AABA01 \\
\hline 3.47 & -- & -- & - & - & -- & -- & -. & - & 04S07W32CBCB01 \\
\hline 82.13 & 20 & 5.0 & D & D & -- & -- & -. & -- & 04S07W33BDBA01 \\
\hline 25.34 & .- & -- & - & 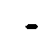 & -- & -- & $\because-$ & -. & 04S06W 16AAAA02 \\
\hline
\end{tabular}


Table 1. Records of selected wells by basin, Northern Rocky Mountains intermontane basins, Montana (Continued)

\begin{tabular}{|c|c|c|c|c|c|c|c|c|c|}
\hline Well number & $\begin{array}{c}\text { Altitude } \\
\text { of land } \\
\text { surface } \\
\text { (ft) }\end{array}$ & $\begin{array}{l}\text { Date well } \\
\text { constructed }\end{array}$ & $\begin{array}{c}\text { Goologic } \\
\text { unit }\end{array}$ & $\begin{array}{c}\text { Depth } \\
\text { of } \\
\text { woll } \\
\text { (ft) }\end{array}$ & $\begin{array}{c}\text { Dlam- } \\
\text { oter } \\
\text { of } \\
\text { casing } \\
\text { (In.) }\end{array}$ & $\begin{array}{c}\text { Type } \\
\text { of } \\
\text { finish }\end{array}$ & $\begin{array}{c}\text { Top of } \\
\text { open } \\
\text { Inter- } \\
\text { val } \\
\text { (ft) }\end{array}$ & $\begin{array}{c}\text { Primary } \\
\text { uee of } \\
\text { water }\end{array}$ & $\begin{array}{l}\text { Date water } \\
\text { loval } \\
\text { measured }\end{array}$ \\
\hline \multicolumn{10}{|c|}{ Beaverhead Valley-Continued } \\
\hline 04S06W16DDDD02 & 4,734 & $09--64$ & Ts & 57 & 1.3 & $\mathrm{O}$ & -- & $\mathbf{U}$ & 09-19-91 \\
\hline 04S06W26ABB 02 & 4,775 & --64 & Qal & 61 & 1.3 & 0 & -- & $\mathbf{U}$ & $09-19-91$ \\
\hline 04S06W27BAAA01 & 4,781 & $03-06-63$ & Ts & 145 & 1.3 & 0 & - & $\mathbf{U}$ & $09-19-91$ \\
\hline 04S06W32ABBA02 & 4,770 & -- & QTsu & 48 & 1.3 & - & -- & $\mathbf{U}$ & $06-26-91$ \\
\hline 04S06W35BBBB01 & 4,900 & $04-17-63$ & Ts & 170 & 1.3 & $\mathrm{P}$ & - & $\mathbf{U}$ & $06-26-91$ \\
\hline 04S05W11DDDD01 & 5,545 & $06-16-81$ & Ts & 145 & 6.0 & $\mathbf{P}$ & 80 & $\mathrm{U}$ & 09-18-91 \\
\hline 04SO5W12CCBB01 & 5,560 & $05-13-75$ & Ts & 36 & 6.0 & $\mathrm{P}$ & 32 & $\mathbf{H}$ & $09-18-91$ \\
\hline 04S05W16DDBC01 & 5,090 & $12-12-84$ & Ts & 175 & 6.0 & $\mathbf{S}$ & 170 & $\mathbf{H}$ & 09-18-91 \\
\hline 04S05W16DDCD01 & 5,090 & $05-02-75$ & Ts & 99 & 6.0 & $\mathbf{P}$ & 92 & $\mathbf{H}$ & $09-20-91$ \\
\hline 04S05W18CAAC01 & 4,790 & $12-19-76$ & Qal & 41 & 6.0 & $\mathbf{p}$ & 28 & $\mathbf{H}$ & $09-18-91$ \\
\hline 04S05W27DBAB01 & 5,060 & $12-04-89$ & Ts & 400 & 13.0 & $\mathbf{P}$ & 40 & $\mathbf{P}$ & $06-27-91$ \\
\hline 04S05W27DBBC01 & 5,055 & $11-28-89$ & Ts & 225 & 12.8 & $\mathbf{X}$ & 81 & $\mathbf{P}$ & $06-27-91$ \\
\hline 04S05W27DBD 01 & 5,070 & -- & Qal & 70 & 12.0 & - & -- & $\mathbf{P}$ & $06-27-91$ \\
\hline 04S05W27DBD 02 & 5,075 & $12-05-89$ & Qal & 90 & 13.0 & $\mathbf{P}$ & 40 & $\mathbf{P}$ & $06-27-91$ \\
\hline $04 \mathrm{~S} 05 \mathrm{~W} 32 \mathrm{DBCC} 01$ & 4,835 & $07-03-74$ & Qal & 49 & 6.0 & o & -- & $\mathbf{H}$ & $09-19-91$ \\
\hline 04S04W19DDDC01 & 5,690 & $09-10-64$ & Qal & 70 & 6.0 & o & -- & $\mathbf{H}$ & $09-19-91$ \\
\hline 04S04W30BCBB01 & 5,480 & $08-23-73$ & Qal & 34 & 6.0 & $\mathbf{P}$ & 28 & $\mathbf{U}$ & $09-19-91$ \\
\hline 05S07W12AAAA01 & 4,788 & -- & QTsu & 36 & 1.3 & - & -- & $\mathbf{U}$ & $06-26-91$ \\
\hline 05S06W07DCDD01 & 4,980 & -- & Ts & 93 & 1.3 & - & -. & $\mathbf{U}$ & $06-26-91$ \\
\hline 05S06W10BCCA01 & 5,065 & $06-\quad-65$ & Ts & 200 & 1.3 & $\mathrm{~T}$ & 197 & $\mathbf{U}$ & $06-25-91$ \\
\hline 05S06W19BDDD02 & 5,015 & -- & Qal & 150 & 6.0 & - & -- & $\mathbf{s}$ & $06-25-91$ \\
\hline 05S05W02DDAA01 & 5,090 & $04-29-89$ & Ts & 100 & 5.0 & $\mathbf{P}$ & 80 & $\mathbf{H}$ & 09-19-91 \\
\hline 05S05W03CBBA01 & 4,940 & $04-29-82$ & Ts & 55 & 6.0 & $\mathbf{P}$ & 48 & $\mathbf{H}$ & $09-19-91$ \\
\hline 05S05W03CBBB01 & 4,935 & $07-23-88$ & Ts & 108 & 6.0 & $\mathbf{P}$ & 89 & C & 09-19-91 \\
\hline 05S05W05CCAA01 & 4,869 & $01-05-91$ & Ts & 73 & 6.0 & $\mathbf{P}$ & 66 & $\mathbf{H}$ & $09-18-91$ \\
\hline 05S05W05CCCA01 & 4,890 & $10-21-81$ & Ts & 95 & 6.0 & $\mathrm{P}$ & 88 & $\mathbf{S}$ & $09-18-91$ \\
\hline 05S05W09DBDA01 & 4,945 & $02-01-79$ & Ts & 160 & 6.0 & $\mathbf{P}$ & 153 & $\mathbf{H}$ & $09-19-91$ \\
\hline 05S05W25BAAA01 & 5,000 & $09-12-77$ & Qal & 41 & 6.0 & $\mathbf{P}$ & 36 & $\mathbf{U}$ & $09-20-91$ \\
\hline 05S05W25BBBB01 & 5,040 & $11-24-78$ & Ts & 101 & 6.0 & $\mathbf{P}$ & 91 & $\mathbf{U}$ & $09-20-91$ \\
\hline 05S04W19BABA01 & 4,992 & $06-30-85$ & Ts & 220 & 6.0 & $\mathbf{P}$ & 80 & $\mathbf{H}$ & $09-19-91$ \\
\hline 05S04W19BABB01 & 4,991 & $09-13-77$ & Ts & 220 & - & - & -- & $\mathbf{H}$ & 09-19-91 \\
\hline 05S04W19BABD01 & 4,993 & $01-01-20$ & Qal & 25 & -- & - & -- & $\mathbf{U}$ & 09-19-91 \\
\hline 05S04W28BBAB01 & 5,150 & $05-01-84$ & Ts & 120 & 6.0 & $\mathbf{P}$ & 60 & $\mathbf{U}$ & $09-20-91$ \\
\hline 05S04W28BBAB02 & 5,150 & $02-16-78$ & Ts & 48 & 6.0 & $\mathbf{P}$ & 42 & $\mathbf{S}$ & - \\
\hline 05S04W28DDBB01 & 5,170 & $02-11-78$ & Ts & 60 & 6.0 & $\mathbf{P}$ & 54 & $\mathbf{H}$ & $09-20-91$ \\
\hline
\end{tabular}


Table 1. Records of selected wells by basin, Northern Rocky Mountains intermontane basins, Montana (Continued)

\begin{tabular}{|c|c|c|c|c|c|c|c|c|c|}
\hline $\begin{array}{c}\text { Water level } \\
\text { (tt) }\end{array}$ & $\begin{array}{c}\text { Dle- } \\
\text { charge } \\
\text { (gal/ } \\
\text { min) }\end{array}$ & $\begin{array}{c}\text { Speclfic } \\
\text { capacity } \\
\text { [(gel } \\
\text { min)/ft] }\end{array}$ & $\begin{array}{l}\text { Source } \\
\text { of dis- } \\
\text { charge } \\
\text { data }\end{array}$ & $\begin{array}{l}\text { Typo } \\
\text { of log } \\
\text { avell- } \\
\text { able }\end{array}$ & $\begin{array}{c}\text { Date } \\
\text { water- } \\
\text { quallty } \\
\text { peremater } \\
\text { moagured }\end{array}$ & $\begin{array}{c}\text { Specific } \\
\text { conduc- } \\
\text { tance } \\
(\mu \mathrm{S} / \mathrm{cm})\end{array}$ & $\begin{array}{c}\text { pH } \\
\text { (stan- } \\
\text { dard } \\
\text { units) }\end{array}$ & $\begin{array}{l}\text { Tem- } \\
\text { per- } \\
\text { ature } \\
\text { (') }\end{array}$ & Well number \\
\hline
\end{tabular}

Beaverhead Valley-Continued

\begin{tabular}{|c|c|c|c|c|c|c|c|c|c|}
\hline 25.96 & -- & -- & - & - & -- & -- & -- & -- & 04S06W 16DDDD02 \\
\hline 24.89 & - & -- & - & - & -- & -- & -- & -- & 04S06W26ABB 02 \\
\hline 43.98 & -- & -- & - & D & -- & -- & -- & -- & 04S06W27BAAA01 \\
\hline 43.09 & - & -- & - & - & -- & -- & -. & -- & 04S06W32ABBA02 \\
\hline+12.50 & -. & -- & - & D & $06-26-91$ & 862 & -- & -- & 04S06W35BBBB01 \\
\hline 26.02 & 30 & .3 & D & D & -- & -- & -. & -- & 04S05W11DDDD01 \\
\hline 25.28 & 13 & -- & D & D & $09-18-91$ & 258 & - & 9.0 & 04S05W 12 CCBB01 \\
\hline 6.50 & 60 & .9 & D & D & $09-18-91$ & 456 & -- & 12.5 & 04S05W16DDBC01 \\
\hline$* 46$ & 14 & -- & D & D & $09-20-91$ & 481 & -- & 11.0 & 04S05W 16DDCD01 \\
\hline 5.08 & 10 & 1.4 & D & $\mathbf{D}$ & $09-18-91$ & 409 & -- & 9.5 & 04S05W18CAAC01 \\
\hline 123.34 & 500 & 2.1 & D & D & -- & -- & -- & -- & 04S05W27DBAB0I \\
\hline 145.13 & 30 & -- & D & D & - & -- & -- & -- & 04S05W27DBBC01 \\
\hline 5.45 & -- & -- & - & - & - & - & -- & -- & 04S05W27DBD 01 \\
\hline 4.68 & 150 & 3.5 & D & D & -- & -- & -- & -. & 04S05W27DBD 02 \\
\hline 9.37 & 40 & -- & D & D & -- & -- & -- & -- & 04S05W32DBCC01 \\
\hline 46.98 & 20 & 2.9 & D & D & $09-19-91$ & 230 & -- & -- & 04S04W 19DDDC01 \\
\hline 6.90 & 30 & 2.0 & D & D & -- & -- & -- & -- & 04S04W30BCBB0 1 \\
\hline 20.62 & -- & -- & - & - & -- & -- & .. & -- & 05S07W12AAAA01 \\
\hline 78.32 & -- & - & - & - & -- & -- & -- & -- & 05S06W07DCDD01 \\
\hline 144.95 & -- & -- & - & - & -- & -- & -- & -- & 05S06W 10BCCA01 \\
\hline 53.97 & -- & -- & - & - & $06-25-91$ & 480 & -- & 11.5 & 05S06W 19BDDD02 \\
\hline 19.36 & 30 & .7 & D & D & $09-19-91$ & 675 & -- & -- & 05S05W02DDAA01 \\
\hline 12.35 & 50 & - & D & D & $09-19-91$ & 634 & -- & 12.5 & 05S05W03CBBA01 \\
\hline 6.62 & 62 & 5.2 & D & D & $09-19-91$ & 404 & - & 10.0 & 05S05W03CBBB01 \\
\hline 45.57 & 50 & 5.0 & D & D & -- & -- & -- & -- & 05S05W05CCAA01 \\
\hline 80.10 & 15 & -- & D & D & -- & -- & -- & -- & 05S05W05CCCA01 \\
\hline 73.03 & 16 & .2 & D & D & $09-19-91$ & 920 & -- & 10.0 & 05S05W09DBDA01 \\
\hline 21.50 & 28 & 1.3 & D & D & - & - & -- & -- & 05S05W25BAAA01 \\
\hline $\mathbf{7 7 . 7 0}$ & 20 & -- & D & D & -- & -- & - & -- & 05S05W25BBBB01 \\
\hline 21.13 & 1 & -- & D & $\mathbf{D}$ & $09-19-91$ & 480 & -- & -- & 05S04W19BABA01 \\
\hline 4.37 & -- & -- & - & $\mathbf{D}$ & 09-19-91 & 338 & -- & 8.5 & 05S04W 19BABB01 \\
\hline 7.65 & -- & -- & - & - & -- & -. & - & -- & 05S04W19BABD01 \\
\hline 20.38 & 20 & .3 & D & D & -- & -- & -. & -- & 05S04W28BBAB01 \\
\hline- & 8 & .6 & D & D & $09-20-91$ & 764 & -- & 11.5 & 05S04W28BBAB02 \\
\hline 15.10 & 32 & .8 & D & D & $09-20-91$ & 645 & -- & -- & 05S04W28DDBB01 \\
\hline
\end{tabular}


Table 1. Records of selected wells by basin, Northern Rocky Mountains intermontane basins, Montana (Continued)

\begin{tabular}{|c|c|c|c|c|c|c|c|c|c|}
\hline Woll number & $\begin{array}{l}\text { Alttude } \\
\text { of land } \\
\text { surfece } \\
\text { (ft) }\end{array}$ & $\begin{array}{l}\text { Date well } \\
\text { conetructod }\end{array}$ & $\begin{array}{c}\text { Geologic } \\
\text { unit }\end{array}$ & $\begin{array}{c}\text { Depth } \\
\text { of } \\
\text { well } \\
\text { (fi) }\end{array}$ & $\begin{array}{l}\text { Dlam- } \\
\text { otor } \\
\text { of } \\
\text { coeling } \\
\text { (In.) }\end{array}$ & $\begin{array}{l}\text { Type } \\
\text { of } \\
\text { finish }\end{array}$ & $\begin{array}{l}\text { Top of } \\
\text { open } \\
\text { Inter- } \\
\text { vel } \\
\text { (fi) }\end{array}$ & $\begin{array}{l}\text { Primary } \\
\text { uee of } \\
\text { woter }\end{array}$ & $\begin{array}{c}\text { Dato wath } \\
\text { level } \\
\text { meesure }\end{array}$ \\
\hline \multicolumn{10}{|c|}{ Beaverhead Valley-Continued } \\
\hline 06S10W29DDCD01 & 6,065 & $04-20-76$ & Ts & 190 & 6.0 & o & -- & $\mathbf{N}$ & $05-23-91$ \\
\hline 06S09W14BDCD0I & 5,188 & $11-22-83$ & Ts & 125 & 6.0 & $\mathbf{P}$ & 109 & $\mathbf{S}$ & $06-21-91$ \\
\hline 06S09W15BBAB01 & 5,320 & $05-30-89$ & Ts & 190 & 6.0 & $\mathbf{P}$ & 180 & $\mathbf{s}$ & $06-21-91$ \\
\hline 06S08W12DCBA02 & 4,957 & --65 & Ts & - & 1.3 & $\mathbf{T}$ & -- & $\mathbf{U}$ & $06-20-91$ \\
\hline 06S08W13CABD01 & 5,025 & $10-19-79$ & Ts & 148 & 6.0 & $\mathbf{P}$ & 141 & $\mathbf{H}$ & $06-13-91$ \\
\hline 06S08W23DAC 02 & 5,000 & --64 & Ts & 102 & 1.3 & - & -- & $\mathbf{U}$ & $06-20-91$ \\
\hline 06S08W26CCCA02 & 5,008 & $04-09-65$ & Ts & 51 & 1.3 & $\mathbf{T}$ & 48 & $\mathbf{U}$ & $06-12-91$ \\
\hline 06S07W06AADA01 & 4,934 & $12-03-64$ & Ts & 107 & 1.3 & $\mathbf{T}$ & 104 & $\mathbf{U}$ & $06-20-91$ \\
\hline 06S07W16BBBB01 & 5,166 & $11-18-66$ & Ts & 220 & 6.0 & - & -- & $\mathbf{H}$ & $06-13-91$ \\
\hline 06S07W16DBCB01 & 5,262 & $08-29-90$ & Ts & 400 & 4.0 & $\mathbf{P}$ & 200 & $\mathbf{S}$ & $06-20-91$ \\
\hline 06S07W21ABCB01 & 5,242 & -- & QTsu & 135 & 6.0 & - & -- & $\mathbf{s}$ & $06-20-91$ \\
\hline 06S07W28DDAD01 & 5,318 & $11-13-75$ & Ts & 108 & 6.0 & $\mathbf{P}$ & 102 & $\mathbf{H}$ & $06-12-91$ \\
\hline 06S07W34ABCC01 & 5,352 & $12-05-73$ & Ts & 80 & 6.0 & $\mathbf{P}$ & 75 & $\mathbf{S}$ & $06-12-91$ \\
\hline 06S04W05AACB01 & 5,075 & $08-12-84$ & Ts & 90 & 6.0 & o & -- & $\mathbf{H}$ & $09-20-91$ \\
\hline 06S04W06ACDA01 & 5,090 & $12-11-76$ & Ts & 64 & 6.0 & $\mathbf{P}$ & 51 & $\mathbf{U}$ & $09-20-91$ \\
\hline 06S04W06DBAB0I & 5,130 & $12-06-76$ & Ts & 170 & 6.0 & $\mathbf{P}$ & 153 & $\mathbf{H}$ & $09-20-91$ \\
\hline 06S04W10CDCC01 & 5,170 & $08-10-83$ & Qal & 81 & 6.0 & $\mathbf{P}$ & 75 & $\mathbf{H}$ & $09-20-91$ \\
\hline 06S04W15CDDC01 & 5,190 & $09-28-79$ & Qal & 58 & 6.0 & $\mathbf{P}$ & 48 & $\mathbf{H}$ & $09-20-91$ \\
\hline 06S04W28ADDD01 & 5,213 & $05-03-80$ & Qal & 43 & 6.0 & $\mathbf{P}$ & 35 & $\mathbf{H}$ & $09-20-91$ \\
\hline 06S04W28BDAA01 & 5,210 & -- & Qal & 43 & - & - & -- & $\mathbf{H}$ & $09-20-91$ \\
\hline 07S10W15CBCC01 & 5,620 & .. & Ts & -- & 6.0 & - & -- & $\mathbf{s}$ & $05-22-91$ \\
\hline 07S10W22AAD 01 & 5,548 & -- & QTsu & 145 & -- & - & -- & I & $05-22-91$ \\
\hline 07S10W24BCCA01 & 5,468 & $10-28-76$ & Ts & 55 & 6.0 & $\mathbf{P}$ & 48 & $\mathbf{U}$ & $05-23-91$ \\
\hline 07S10W24BCCA02 & 5,468 & $09-07-90$ & Ts & 90 & 6.0 & $\mathbf{S}$ & 84 & $\mathbf{H}$ & $05-23-91$ \\
\hline 07S10W24BDB 01 & 5,480 & $02-04-80$ & QTsu & 192 & 6.0 & o & - & $\mathbf{s}$ & $05-22-91$ \\
\hline 07S09W23BDAC01 & 5,135 & $03-26-48$ & QTsu & 124 & 10.0 & - & -- & $\mathbf{P}$ & -- \\
\hline 07S09W29CCCC01 & 5,280 & $12-\quad-79$ & QTsu & 185 & 20.0 & $\mathbf{P}$ & 10 & I & $05-22-91$ \\
\hline 07S09W30DDBB01 & 5,298 & $-\quad-54$ & QTsu & 250 & 6.0 & - & -. & $\mathbf{U}$ & $05-22-91$ \\
\hline 07S09W32ABCC01 & 5,236 & -- & QTsu & 200 & -- & - & -- & I & $05-22-91$ \\
\hline 07S09W32ACDC01 & 5,225 & $-\quad-87$ & QTsu & 200 & 20.0 & $\mathbf{P}$ & 20 & I & $05-22-91$ \\
\hline 07S09W33ACBA01 & 5,180 & $10-20-89$ & Qal & 112 & 20.0 & $\mathbf{P}$ & 22 & I & $05-22-91$ \\
\hline 07S09W33ADDA01 & 5,172 & -- & Qal & 100 & 6.0 & - & -- & I & $05-21-91$ \\
\hline 07S09W33ADDD01 & 5,175 & $10-23-77$ & Qal & 70 & 20.0 & $\mathbf{P}$ & 10 & $\mathbf{I}$ & $05-16-91$ \\
\hline 07S08W03BDCA02 & 5,030 & --65 & Qal & 41 & 1.3 & o & -- & $\mathbf{U}$ & $06-12-91$ \\
\hline 07S08W10BBAC01 & 5,043 & -- & QTsu & 44 & 6.0 & - & -- & $\mathbf{H}$ & $06-12-91$ \\
\hline
\end{tabular}


Table 1. Records of selected wells by basin, Northern Rocky Mountains intermontane basins, Montana (Continued)

\begin{tabular}{|c|c|c|c|c|c|c|c|c|c|}
\hline $\begin{array}{l}\text { Water level } \\
\text { (ft) }\end{array}$ & $\begin{array}{c}\text { Dis- } \\
\text { charge } \\
\text { (gall } \\
\text { m!n) }\end{array}$ & $\begin{array}{c}\text { Speclfic } \\
\text { capaclty } \\
\text { [(gal } \\
m(n) / f t]\end{array}$ & $\begin{array}{l}\text { Source } \\
\text { of dla- } \\
\text { charge } \\
\text { data }\end{array}$ & $\begin{array}{l}\text { Type } \\
\text { of log } \\
\text { avall- } \\
\text { able }\end{array}$ & $\begin{array}{c}\text { Date } \\
\text { water- } \\
\text { quallty } \\
\text { parameter } \\
\text { measured }\end{array}$ & $\begin{array}{c}\text { Specific } \\
\text { conduc- } \\
\text { tence } \\
(\mu \mathrm{S} / \mathrm{cm})\end{array}$ & $\begin{array}{l}\text { pH } \\
\text { (stan- } \\
\text { dard } \\
\text { units) }\end{array}$ & $\begin{array}{l}\text { Tom- } \\
\text { per- } \\
\text { ature } \\
\left({ }^{\circ} \mathrm{C}\right)\end{array}$ & Well number \\
\hline
\end{tabular}

\begin{tabular}{|c|c|c|c|c|c|c|c|c|c|}
\hline \\
\hline 98.99 & 25 & .3 & D & D & -- & - & -- & -- & 06S10W29DDCD01 \\
\hline 62.63 & 20 & 5.0 & $\mathrm{D}$ & D & -- & - & - & -- & 06S09W14BDCD01 \\
\hline 145.33 & 20 & $\cdots$ & D & D & -. & -. & - & -- & 06S09W 15BBAB01 \\
\hline 6.81 & -- & -- & - & - & -- & -- & -- & -- & 06S08W12DCBA02 \\
\hline 72.50 & 30 & -- & $\mathrm{D}$ & D & $06-13-91$ & 729 & 7.9 & 12.5 & 06S08W 13CABD01 \\
\hline 26.94 & -. & -- & - & - & -- & -- & -- & -- & 06S08W23DAC 02 \\
\hline 28.87 & -- & -- & - & - & -- & - & -- & -- & 06S08W26CCCA02 \\
\hline 41.60 & -- & -- & - & - & -- & -- & -- & -- & 06S07W06AADA01 \\
\hline 84.11 & 12 & -- & $\mathbf{D}$ & $\mathbf{D}$ & $06-13-91$ & 368 & 7.8 & 12.5 & 06S07W16BBBB01 \\
\hline 129.94 & 90 & 1.3 & D & D & -- & - & - & -- & 06S07W16DBCB01 \\
\hline 67.68 & -- & -- & - & - & -- & -- & -- & -- & 06S07W21ABCB01 \\
\hline 53.88 & 18 & -- & D & D & -- & -- & -- & -- & 06S07W28DDAD01 \\
\hline 18.28 & 30 & 6.0 & D & D & - & - & -- & $\cdots$ & 06S07W34ABCC01 \\
\hline 3.34 & -- & -- & - & D & $09-20-91$ & 655 & -- & - & 06S04W05AACB01 \\
\hline 10.81 & 10 & 2.0 & $\mathbf{D}$ & $\mathrm{D}$ & - & -- & - & $\cdots$ & 06S04W06ACDA01 \\
\hline 55.89 & 20 & 4.0 & D & D & $09-20-91$ & 600 & - & 10.0 & 06S04W06DBAB01 \\
\hline 6.59 & 25 & -- & D & D & $09-20-91$ & 525 & -- & -- & 06S04W 10CDCC01 \\
\hline 30.35 & 20 & .9 & D & D & $09-20-91$ & 711 & $\ldots$ & -. & 06S04W15CDDC01 \\
\hline 4.65 & 30 & 1.2 & D & D & -- & -- & -- & -- & 06S04W28ADDD01 \\
\hline 18.40 & -- & -- & - & - & 09-20-91 & 680 & -- & 13.5 & 06S04W28BDAA01 \\
\hline 17.57 & -. & -- & - & - & -- & -. & -. & -- & 07S 10W15CBCC01 \\
\hline 8.70 & 15 & 1.6 & $\mathbf{S}$ & - & $05-22-91$ & 669 & -- & 9.5 & 07S10W22AAD 01 \\
\hline 26.21 & 15 & -- & D & D & -- & - & - & -- & 07S 10W24BCCA01 \\
\hline 28.53 & 20 & .4 & D & D & 05-23-91 & 663 & -- & 10.5 & 07S10W24BCCA02 \\
\hline+46.62 & 50 & 15 & $\mathbf{D}$ & $\mathbf{D}$ & - & - & -- & -- & 07S10W24BDB 01 \\
\hline$\cdots$ & -- & -- & - & D & $08-22-91$ & 700 & 7.3 & 9.5 & 07S09W23BDAC01 \\
\hline 9.97 & 600 & 6.5 & D & D & -- & - & -- & $\cdots$ & 07S09W 29CCCC01 \\
\hline 3.08 & -. & -- & - & - & -. & -. & -- & -. & 07S09W30DDBB01 \\
\hline 17.31 & -- & -- & - & - & - & -- & -- & $\cdots$ & 07S09W32ABCC01 \\
\hline 19.08 & -- & -- & - & D & -- & -- & - & -- & 07SO9W32ACDC01 \\
\hline 15.29 & 1,200 & 32 & D & D & -- & -- & -- & -- & 07S09W33 ACBA01 \\
\hline 8.01 & -- & -- & - & D & -- & - & -. & $\cdots$ & 07S09W33ADDA01 \\
\hline 8.30 & 2,200 & 180 & D & D & -- & -- & -- & -- & 07S09W33ADDD01 \\
\hline 14.98 & -- & -- & - & - & -- & -- & -- & - & 07S08W03BDCA02 \\
\hline 23.78 & 9 & 460 & $\mathbf{s}$ & - & $06-12-91$ & 766 & 7.4 & 11.5 & 07S08W 10BBAC01 \\
\hline
\end{tabular}


Table 1. Records of selected wells by basin, Northern Rocky Mountains intermontane basins, Montana (Continued)

\begin{tabular}{|c|c|c|c|c|c|c|c|c|c|}
\hline Well number & $\begin{array}{c}\text { Altitude } \\
\text { of land } \\
\text { surface } \\
\text { (ft) }\end{array}$ & $\begin{array}{l}\text { Date well } \\
\text { constructed }\end{array}$ & $\begin{array}{c}\text { Geologic } \\
\text { unit }\end{array}$ & $\begin{array}{c}\text { Depth } \\
\text { of } \\
\text { well } \\
\text { (ft) }\end{array}$ & $\begin{array}{c}\text { Diam. } \\
\text { oter } \\
\text { of } \\
\text { casing } \\
\text { (in.) }\end{array}$ & $\begin{array}{l}\text { Type } \\
\text { of } \\
\text { finish }\end{array}$ & $\begin{array}{l}\text { Top of } \\
\text { open } \\
\text { inter- } \\
\text { val } \\
\text { (ft) }\end{array}$ & $\begin{array}{l}\text { Primary } \\
\text { use of } \\
\text { water }\end{array}$ & $\begin{array}{c}\text { Date water } \\
\text { lovel } \\
\text { measured }\end{array}$ \\
\hline \multicolumn{10}{|c|}{ Beaverhead Valley-Continued } \\
\hline 07S08W1 1BBCB01 & 5,100 & --64 & Ts & 91 & 1.3 & - & -- & $\mathbf{U}$ & $06-12-91$ \\
\hline 07S08W17DDC 02 & 5,110 & --65 & Ts & 50 & 1.3 & 0 & -- & $\mathbf{U}$ & $06-11-91$ \\
\hline 07S08W18CDCC01 & 5,095 & $03-02-63$ & Qal & 61 & 14.0 & $\mathbf{P}$ & 20 & $\mathbf{P}$ & $06-11-91$ \\
\hline 08S09W01CCCC01 & 5,237 & --66 & Ts & 47 & 6.0 & o & -- & $\mathbf{U}$ & $07-11.91$ \\
\hline 08S09W03DDCD01 & 5,200 & $05-04-88$ & QTsu & 41 & 6.0 & o & -- & $\mathbf{H}$ & $05-21-91$ \\
\hline 08S09W14CDD 01 & 5,276 & -. & QTsu & 405 & -- & - & -- & I & $05-16-91$ \\
\hline 08S09W17CAAA0I & 5,253 & 08-08-77 & QTsu & 455 & 8.0 & $\mathbf{P}$ & 395 & $\mathbf{N}$ & $05-23-91$ \\
\hline 08S09W19ADBB01 & 5,257 & $02-22-72$ & QTsu & 62 & 6.0 & 0 & -- & $\mathbf{P}$ & $05-22-91$ \\
\hline 08S09W23ACBD01 & 5,290 & $03-19-80$ & QTsu & 300 & 16.0 & $\mathbf{P}$ & 55 & I & $05-16-91$ \\
\hline 08S09W23BDB 01 & 5,280 & $02-\quad-80$ & QTsu & 300 & 16.0 & $\mathbf{P}$ & 80 & $\mathbf{I}$ & $05-16-91$ \\
\hline 08S09W24CCCC01 & 5,330 & $01-01-76$ & QTsu & 400 & 20.0 & $\mathbf{P}$ & 115 & $\mathbf{I}$ & -- \\
\hline 08S09W25ADBA01 & 5,382 & $06-18-75$ & QTsu & 700 & 16.0 & $\mathbf{P}$ & 150 & $\mathbf{I}$ & $05-16-91$ \\
\hline 08S09W25BAAA01 & 5,353 & -- & QTsu & -- & -- & - & -- & I & $05-16-91$ \\
\hline 08S09W26ABAB01 & 5,318 & $-\quad-76$ & QTsu & 400 & 20.0 & $\mathbf{P}$ & 115 & I & 05-16-91 \\
\hline 08S09W26BBD 01 & 5,322 & $10-30-78$ & QTsu & 415 & 16.0 & $\mathbf{P}$ & 75 & $\mathbf{I}$ & $05-16-91$ \\
\hline 08S08W07DDDC01 & 5,313 & $08-30-77$ & QTsu & 300 & 20.0 & $\mathbf{P}$ & 30 & I & 05-16-91 \\
\hline 08S08W07DDDD01 & 5,313 & $09-\quad-51$ & Qal & 186 & 6.0 & - & -- & I & $05-16-91$ \\
\hline 08S08W20ACCA01 & 5,396 & -- & Qal & 210 & -- & - & -- & I & $05-20-91$ \\
\hline 08S08W20DDCC01 & 5,402 & -- & Qal & 140 & -- & - & -- & I & $05-20-91$ \\
\hline 08S08W29CDAD01 & 5,442 & $05-14-52$ & Qal & 172 & 20.0 & $\mathbf{P}$ & 35 & I & $05-20-91$ \\
\hline 08S08W32DACA01 & 5,483 & $\mid 1-01-51$ & Qal & 165 & 24.0 & $\mathbf{s}$ & 100 & I & $05-20-91$ \\
\hline 08S08W32DBBA01 & 5,478 & $05-15-62$ & QTsu & 185 & 20.0 & $\mathbf{P}$ & 35 & I & $05-20-91$ \\
\hline 09S08W04CAAC01 & 5,536 & $12-16-52$ & QTsu & 150 & 20.0 & $\mathbf{P}$ & 65 & $\mathbf{I}$ & $05-20-91$ \\
\hline 09S08W07DBCC01 & 5,666 & 03-09-64 & QTsu & 376 & 20.0 & $\mathbf{P}$ & 140 & I & $05-21-91$ \\
\hline 09S08W07DCBB01 & 5,684 & $12-21-63$ & QTsu & 350 & 20.0 & $\mathbf{P}$ & 120 & $\mathbf{I}$ & $05-21-91$ \\
\hline \multicolumn{10}{|c|}{ Big Hole Basin } \\
\hline $02 N 13 W 16 D D C \quad 01$ & 6,200 & $09-19-89$ & TKi & 300 & 6.0 & $\mathbf{P}$ & 260 & $\mathbf{H}$ & $07-19-91$ \\
\hline 02N13W30CDAA01 & 6,040 & $05-12-60$ & Ts & 73 & 6.0 & - & -- & $\mathbf{H}$ & $07-19-91$ \\
\hline 01N15W14BCAA01 & 6,315 & $11-05-54$ & $\mathrm{Qg}$ & 99 & 6.0 & $\mathbf{x}$ & 80 & $\mathbf{H}$ & $07-18-91$ \\
\hline 01N15W34DADC01 & 6,125 & $05-19-60$ & Ts & 75 & 6.0 & $\mathrm{x}$ & 55 & $\mathbf{S}$ & $07-18-91$ \\
\hline 01N15W34DDAB01 & 6,130 & --41 & Ts & 100 & 4.0 & - & -- & $\mathbf{H}$ & $07-18-91$ \\
\hline 01N14W15DDDD01 & 6,030 & $01-30-62$ & Qal & 41 & 6.0 & $\mathbf{P}$ & 36 & $\mathbf{H}$ & $07-18-91$ \\
\hline $01 N 13 W 04 B C B C 01$ & 5,850 & $07-06-89$ & Qal & 48 & 6.0 & $\mathbf{P}$ & 40 & $\mathbf{H}$ & $07-19-91$ \\
\hline 01S16W10CBCA01 & 6,325 & -- & Qal & -- & -- & - & -- & $\mathbf{H}$ & $07-18-91$ \\
\hline 01S16W34DBDD01 & 6,071 & $04-01-78$ & Qal & 34 & 6.0 & - & -- & $\mathbf{H}$ & $07-17-91$ \\
\hline 01S15W02ADAC01 & 6,055 & $08-01-43$ & Ts & 135 & 5.0 & - & - & $\mathbf{U}$ & $07-18-91$ \\
\hline
\end{tabular}


Table 1. Records of selected wells by basin, Northern Rocky Mountains intermontane basins, Montana (Continued)

\begin{tabular}{|c|c|c|c|c|c|c|c|c|c|}
\hline $\begin{array}{c}\text { Wator lovel } \\
\text { (fi) }\end{array}$ & $\begin{array}{c}\text { Dis- } \\
\text { charge } \\
\text { (gall } \\
\text { min) }\end{array}$ & $\begin{array}{c}\text { Specific } \\
\text { capacity } \\
\text { [(gal/ } \\
\text { min)/ft] }\end{array}$ & $\begin{array}{l}\text { Source } \\
\text { of dls- } \\
\text { charge } \\
\text { data }\end{array}$ & $\begin{array}{l}\text { Typo } \\
\text { of log } \\
\text { avall- } \\
\text { able }\end{array}$ & $\begin{array}{c}\text { Date } \\
\text { water- } \\
\text { quality } \\
\text { parameter } \\
\text { measured }\end{array}$ & $\begin{array}{c}\text { Specific } \\
\text { conduc- } \\
\text { tence } \\
(\mu S / c m)\end{array}$ & $\begin{array}{c}\mathrm{pH} \\
\text { (stan- } \\
\text { dard } \\
\text { unlts) }\end{array}$ & $\begin{array}{l}\text { Tem- } \\
\text { per- } \\
\text { ature } \\
\left({ }^{\circ} \mathrm{C}\right)\end{array}$ & Woll number \\
\hline
\end{tabular}

Beaverhead Valley-Continued

\begin{tabular}{|c|c|c|c|c|c|c|c|c|c|}
\hline 4.60 & -- & .. & - & - & -- & -- & -- & -. & 07S08W11BBCB01 \\
\hline 31.43 & -- & -. & - & - & -- & -- & - & -- & 07S08W17DDC 02 \\
\hline 14.64 & 1,000 & 140 & D & D & -- & -- & -- & -- & 07S08W18CDCC01 \\
\hline 24.83 & -- & -- & - & - & - & -- & - & -- & 08S09W01CCCC01 \\
\hline 18.72 & 12 & 3.2 & $\mathbf{S}$ & D & $05-21-91$ & 636 & -- & 9.0 & 08S09W03DDCD01 \\
\hline 86.80 & -. & -- & - & D & -- & -- & - & -. & 08S09W14CDD 01 \\
\hline 9.97 & 60 & 60 & D & D & -- & -- & -- & -- & 08S09W17CAAA01 \\
\hline 8.66 & 10 & 1.1 & $\mathbf{S}$ & D & $05-22-91$ & 655 & 7.6 & 10.0 & 08S09W19ADBB01 \\
\hline 43.97 & 2,400 & 240 & D & D & - & -- & - & -- & 08S09W23ACBD01 \\
\hline 80.60 & 1,800 & 33 & D & D & - & -- & -- & -- & 08S09W23BDB 01 \\
\hline -- & -- & -- & - & D & $08-21-91$ & 614 & 7.4 & 12.5 & 08S09W24CCCC01 \\
\hline 114.26 & 820 & 3.8 & $D$ & D & -- & -- & -- & -- & 08S09W25ADBA01 \\
\hline 95.76 & -- & - & - & - & - & - & -- & -- & 08S09W25BAAA01 \\
\hline 61.46 & -- & -- & - & D & -- & $\cdots$ & -. & -- & 08S09W26ABAB01 \\
\hline 121.13 & 2,500 & 50 & D & D & - & -- & - & -. & 08S09W26BBD 01 \\
\hline 57.53 & 2,000 & 13 & D & D & - & -- & -- & -- & 08S08W07DDDC01 \\
\hline 59.73 & 2,300 & -- & D & D & -- & -- & -- & -- & 08S08W07DDDD01 \\
\hline 57.13 & -- & -- & - & D & - & -- & -- & - & 08S08W20ACCAOI \\
\hline 28.72 & -- & -- & - & D & - & -- & - & -- & 08S08W20DDCC01 \\
\hline 49.12 & 2,000 & -- & D & D & - & - & -- & - & 08S08W29CDAD01 \\
\hline 54.91 & 2,000 & -- & D & D & - & -- & -- & -- & 08S08W32DACA01 \\
\hline 54.60 & 1,800 & 28 & D & D & -- & -- & -- & -- & 08S08W32DBBA01 \\
\hline 56.27 & 800 & - & D & D & - & -- & - & -- & 09S08W04CAAC01 \\
\hline 178.92 & 980 & 7.4 & D & D & -- & -- & -- & -- & 09S08W07DBCC01 \\
\hline 156.51 & 1,700 & 44 & D & D & -- & -- & -- & -- & 09S08W07DCBB01 \\
\hline
\end{tabular}

Big Hole Basin

\begin{tabular}{|c|c|c|c|c|c|c|c|c|c|}
\hline 109.91 & 12 & .1 & $\mathrm{D}$ & D & -- & - & - & $\cdots$ & 02N13W16DDC 01 \\
\hline 11.70 & 10 & .2 & D & D & -- & -.. & - & -- & 02N13W30CDAA01 \\
\hline 13.80 & -. & -- & - & D & -- & - & - & -- & 01N15W 14BCAA01 \\
\hline 26.30 & $\checkmark 50$ & 1.7 & D & D & - & -- & - & -- & 01N15W34DADC01 \\
\hline 33.89 & .- & -- & - & - & -- & - & -- & -- & 01N15W34DDAB01 \\
\hline 9.40 & 15 & 1.5 & D & D & $10-13-82$ & 129 & 6.9 & 8.0 & 01N14W15DDDD01 \\
\hline 7.08 & 20 & -- & D & D & - & -- & -- & -- & $01 N 13 W 04 B C B C 01$ \\
\hline 21.59 & -. & -. & - & - & -- & -- & - & -. & 01S16W10CBCA01 \\
\hline 5.29 & 4 & 2.5 & $\mathbf{S}$ & - & $09-21-82$ & 141 & 6.4 & 8.0 & 01S16W34DBDD01 \\
\hline 41.88 & $\ldots$ & $\ldots$ & - & D & -- & $\ldots$ & -- & -- & 01S15W02ADAC01 \\
\hline
\end{tabular}


Table 1. Records of selected wells by basin, Northern Rocky Mountains intermontane basins, Montana (Continued)

\begin{tabular}{|c|c|c|c|c|c|c|c|c|c|}
\hline Well number & $\begin{array}{l}\text { Altitude } \\
\text { of land } \\
\text { surface } \\
\text { (fi) }\end{array}$ & $\begin{array}{c}\text { Date weli } \\
\text { constructed }\end{array}$ & $\begin{array}{c}\text { Goologic } \\
\text { unit }\end{array}$ & $\begin{array}{c}\text { Depth } \\
\text { of } \\
\text { well } \\
\text { (fi) }\end{array}$ & $\begin{array}{c}\text { Dlam- } \\
\text { oter } \\
\text { of } \\
\text { casing } \\
\text { (In.) }\end{array}$ & $\begin{array}{c}\text { Type } \\
\text { of } \\
\text { finieh }\end{array}$ & $\begin{array}{l}\text { Top of } \\
\text { open } \\
\text { Inter- } \\
\text { val } \\
\text { (it) }\end{array}$ & $\begin{array}{l}\text { Primary } \\
\text { use of } \\
\text { water }\end{array}$ & $\begin{array}{r}\text { Date wol } \\
\text { lovel } \\
\text { measum }\end{array}$ \\
\hline \multicolumn{10}{|c|}{ Big Hole Basin-Continued } \\
\hline 01S15W04DACC01 & 6,100 & $08-25-81$ & Ts & 136 & 6.0 & 0 & -- & $\mathbf{H}$ & $07-18-91$ \\
\hline 01S15W35BDCC01 & 5,971 & $-90^{\prime}$ & Ts & -- & -- & - & - & $\mathbf{s}$ & 07-18-91 \\
\hline 01S14W18DBAC01 & 5,925 & $-\quad-45$ & Ts & 65 & 6.0 & - & -- & $\mathbf{U}$ & $07-18-91$ \\
\hline 01S14W20AAAB01 & 6,561 & $07-13-82$ & TKi & 525 & 6.0 & $\mathbf{P}$ & 360 & $\mathbf{H}$ & $07-18-91$ \\
\hline 02S17W24DCAA01 & 6,295 & $06-25-65$ & Ts & 250 & 6.0 & $\mathbf{P}$ & 200 & $\mathbf{P}$ & $07-17-91$ \\
\hline 02S17W26BBBB01 & 6,215 & $07-21-73$ & Ts & 61 & 6.0 & $\mathbf{P}$ & 49 & $\mathbf{P}$ & 07-17-91 \\
\hline 02S16W07DAAD01 & 6,180 & $06-16-82$ & Ts & 175 & 6.0 & $\mathbf{P}$ & 168 & $\mathbf{H}$ & $07-17-91$ \\
\hline 02S $15 W 15 \mathrm{CABA} 01$ & 5,995 & $09-24-63$ & Ts & 47 & 6.0 & o & -. & I & $07-18-91$ \\
\hline 02S15W32AABB01 & 6,054 & 09-24-80 & Qal & 42 & 6.0 & $\mathbf{P}$ & 32 & $\mathbf{H}$ & $07-18-91$ \\
\hline 02SI5W33BDDA01 & 6,045 & $07-20-78$ & Qal & 52 & 6.0 & $\mathbf{P}$ & 40 & $\mathbf{P}$ & $07-17-91$ \\
\hline 02S15W33CCAB01 & 6,063 & $12-03-80$ & Ts & 286 & 6.0 & $\mathbf{P}$ & 240 & $\mathbf{H}$ & $07-18-91$ \\
\hline 02S15W34BCCD01 & 6,045 & --69 & Ts & 125 & 6.0 & $\mathrm{x}$ & 50 & $\mathbf{H}$ & $07-17-91$ \\
\hline 02S15W34CCCB01 & 6,060 & $12-29-86$ & Ts & 475 & 6.0 & $\mathbf{P}$ & 180 & H & $07-17-91$ \\
\hline 03S16W09ABBC0I & 6,197 & -- & Qal & 40 & 6.0 & - & - & $\mathbf{H}$ & $07-17-91$ \\
\hline 03S16W31DDAD01 & 6,510 & $10-09-88$ & Ts & 190 & 6.0 & $\mathbf{P}$ & 150 & $\mathbf{H}$ & $07-17-91$ \\
\hline 03S16W31DDDD01 & 6,510 & $10-14-88$ & Ts & 166 & 6.0 & $\mathbf{P}$ & 158 & $\mathbf{H}$ & $07-17-91$ \\
\hline 03S16W36ACDA01 & 6,176 & $09-26-62$ & Qal & 35 & 6.0 & $\mathbf{P}$ & 32 & $\mathbf{S}$ & $07-17-91$ \\
\hline 03S16W36ACDD01 & 6,175 & 07-03-90 & Ts & 99 & 6.0 & $\mathbf{P}$ & 82 & $\mathbf{H}$ & $07-17-91$ \\
\hline 03S $15 W 16 D C C D 01$ & 6,141 & $09-29-75$ & Ts & 205 & 6.0 & $\mathbf{P}$ & 200 & $\mathbf{U}$ & $07-16-91$ \\
\hline 03S15W20DBCC02 & 6,151 & $11-06-70$ & Ts & 72 & 6.0 & $\mathbf{P}$ & 64 & $\mathbf{H}$ & $07-16-91$ \\
\hline 03S15W21DCAB01 & 6,151 & $02-28-67$ & Ts & 40 & 6.0 & $\mathbf{P}$ & 32 & $\mathbf{s}$ & 07-16-91 \\
\hline 03S14W04CACA01 & 6,340 & $08-28-50$ & $\mathrm{Qg}$ & 31 & 6.0 & - & -- & $\mathbf{H}$ & 07-18-91 \\
\hline 04S16W03CBBB01 & 6,363 & $07-22-75$ & Ts & 115 & 6.0 & $\mathbf{P}$ & 105 & I & $07-17-91$ \\
\hline 04S16W06DAAA01 & 6,520 & $-\quad-52$ & Ts & 130 & 4.0 & - & -- & $\mathbf{U}$ & $07-17-91$ \\
\hline 04S16W06DAAD01 & 6,522 & $10-07-88$ & Ts & 139 & 6.0 & $\mathbf{P}$ & 134 & HI & $07-17-91$ \\
\hline 04S15W02CCCB01 & 6,350 & $08-01-82$ & Ts & 235 & 6.0 & $\mathbf{P}$ & 180 & H & $07-16-91$ \\
\hline 04S15W05DBCD01 & 6,196 & $03-02-67$ & Qal & 35 & 6.0 & $\mathbf{P}$ & 28 & $\mathbf{S}$ & $07-16-91$ \\
\hline 04S15W29ADDB0 1 & 6,284 & - & Qal & 60 & 5.0 & - & -- & $\mathbf{S}$ & $07-16-91$ \\
\hline 04S15W29ADDC01 & 6,285 & -. & Qal & 90 & 6.0 & - & -- & H & $07-16-91$ \\
\hline 05S16W16ABBA01 & 6,775 & $10-12-88$ & Ts & 28 & 6.0 & $\mathbf{P}$ & 20 & $\mathbf{H}$ & $07-17-91$ \\
\hline 05S15W10AADB01 & 6,420 & $07-21-70$ & Ts & 95 & 6.0 & o & -- & $\mathbf{H}$ & $07-16-91$ \\
\hline 05S15W17BABA01 & 6,428 & $12-19-80$ & Qal & 41 & 6.0 & $\mathbf{P}$ & 31 & $\mathbf{H}$ & $07-16-91$ \\
\hline 05S15W19DBAC01 & 6,540 & $11-12-48$ & Qal & 45 & 5.0 & - & - & HI & $07-16-91$ \\
\hline 05S15W25BCCB01 & 6,475 & -- & Ts & 42 & -- & - & -- & $\mathbf{H}$ & $07-16-91$ \\
\hline 05S15W36CABD01 & 6,558 & $08-17-72$ & Ts & 36 & 6.0 & $\mathbf{P}$ & 30 & $\mathbf{U}$ & 07-19-91 \\
\hline
\end{tabular}

${ }^{1} 1890$ 
Table 1. Records of selected wells by basin, Northern Rocky Mountains intermontane basins, Montana (Continued)

\begin{tabular}{|c|c|c|c|c|c|c|c|c|c|}
\hline $\begin{array}{c}\text { Water level } \\
\text { (ft) }\end{array}$ & $\begin{array}{c}\text { Die- } \\
\text { charge } \\
\text { (gal/ } \\
\text { min) }\end{array}$ & $\begin{array}{c}\text { Speclfic } \\
\text { capacity } \\
\text { [(gal/ } \\
m(n) / f t]\end{array}$ & $\begin{array}{l}\text { Source } \\
\text { of dis- } \\
\text { charge } \\
\text { data }\end{array}$ & $\begin{array}{l}\text { Type } \\
\text { of log } \\
\text { avall- } \\
\text { able }\end{array}$ & $\begin{array}{c}\text { Date } \\
\text { water- } \\
\text { quellity } \\
\text { parameter } \\
\text { measured }\end{array}$ & $\begin{array}{c}\text { Specific } \\
\text { conduc- } \\
\text { tance } \\
\text { ( } \mu \text { S/cm) }\end{array}$ & $\begin{array}{c}\text { pH } \\
\text { (stan- } \\
\text { dard } \\
\text { units) }\end{array}$ & $\begin{array}{l}\text { Tem- } \\
\text { per* } \\
\text { ature } \\
\left({ }^{\circ} \mathrm{C}\right)\end{array}$ & Woll number \\
\hline
\end{tabular}

Big Hole Basin-Continued

\begin{tabular}{|c|c|c|c|c|c|c|c|c|c|}
\hline 71.16 & 10 & 1.7 & $\mathbf{S}$ & $\mathbf{D}$ & $08-26-82$ & 125 & 6.8 & 7.0 & 01S15W04DACC01 \\
\hline 12.68 & -- & -- & - & - & -- & -- & -- & -- & 01S15W35BDCC01 \\
\hline 13.03 & .- & -- & - & D & 09-13-83 & 169 & 7.2 & 8.5 & 01S14W 18DBAC01 \\
\hline 14.04 & 8 & -- & D & D & $09-01-82$ & 178 & 8.3 & 7.5 & 01SI4W20AAAB01 \\
\hline 76.50 & -- & -- & - & D & -- & -- & -. & -- & 02S17W24DCAA01 \\
\hline 1.84 & 15 & 5.9 & $\mathbf{s}$ & D & $09-21-82$ & 243 & 6.9 & 6.5 & 02S17W26BBBB01 \\
\hline 41.60 & 9 & .2 & $\mathbf{S}$ & D & $09-22-82$ & 167 & 7.1 & 9.0 & 02S16W07DAAD01 \\
\hline 4.63 & 20 & .7 & D & D & -- & -- & -- & -. & 02S 15 W 15CABA01 \\
\hline 12.24 & 10 & 3.2 & $\mathbf{S}$ & D & $09-21-82$ & 104 & 6.6 & 8.0 & 02S15W32AABB01 \\
\hline 4.09 & 15 & 5.0 & D & D & -- & -- & -- & -- & 02S15W33BDDA01 \\
\hline 24.06 & 10 & 1.1 & $\mathrm{~s}$ & D & $08-10-82$ & 141 & 8.0 & 10.0 & 02S $15 W 33 C C A B 01$ \\
\hline 12.40 & 4 & 2.4 & $\mathbf{S}$ & $\mathbf{D}$ & $08-09-82$ & 156 & 7.1 & 8.0 & 02S15W34BCCD01 \\
\hline 10.38 & 250 & 1.2 & D & D & -- & -- & -- & -- & 02S15W34CCCB01 \\
\hline 9.47 & 5 & -- & $\mathbf{s}$ & - & $06-08-83$ & 120 & 7.2 & 6.5 & 03S16W09ABBC01 \\
\hline 140.72 & 25 & -- & $\mathbf{D}$ & $\mathbf{D}$ & -- & -- & -- & -. & 03SI6W31DDAD01 \\
\hline 140.00 & 12 & .5 & D & D & - & -- & -- & -- & 03S16W31DDDD01 \\
\hline 4.64 & 10 & 1.0 & $\mathrm{~s}$ & D & 09-22-82 & 975 & 6.8 & 5.0 & 03S16W36ACDA01 \\
\hline 4.90 & 32 & 1.0 & D & D & -. & - & -- & -- & 03S16W36ACDD01 \\
\hline 23.30 & -- & -- & - & D & -- & - & - & -- & 03S15W 16DCCD01 \\
\hline 15.09 & 30 & 1.5 & $\mathbf{D}$ & D & $08-11-82$ & 188 & 7.1 & 7.0 & 03S15W20DBCC02 \\
\hline 10.10 & 20 & 1.3 & D & D & -- & -- & -- & -- & 03S15W21DCAB01 \\
\hline 9.60 & 4 & -- & D & D & -- & -- & -. & -- & 03S 14W04CACA01 \\
\hline 52.74 & 30 & 10 & D & D & -- & - & -- & -- & 04S16W03CBBB01 \\
\hline 96.59 & -- & -- & - & - & -- & -- & -- & -- & 04S16W06DAAA01 \\
\hline 97.49 & 9 & .2 & D & D & -- & - & - & -- & 04S16W06DAAD01 \\
\hline 135.87 & 7 & .6 & $\mathbf{S}$ & D & $08-24-82$ & 707 & 7.2 & 8.0 & 04S15W02CCCB01 \\
\hline+1.60 & 20 & 2.0 & D & D & -- & -- & -- & -. & 04S15W05DBCD01 \\
\hline 5.49 & -- & -- & - & - & -- & -- & - & -- & 04S15W29ADDB01 \\
\hline 7.95 & -- & -- & - & - & -- & -- & -- & -- & 04S15W29ADDC01 \\
\hline 5.64 & 4 & .3 & D & D & -- & -- & -- & -- & 05S16W 16ABBA01 \\
\hline 54.23 & 13 & 83 & $\mathrm{~S}$ & D & $08-24-82$ & 302 & 7.5 & 10.0 & 05S15W 10AADB01 \\
\hline 6.56 & 7 & 1.7 & $\mathbf{s}$ & D & $08-24-82$ & 185 & 6.8 & 6.0 & 05S15W 17BABA01 \\
\hline$*+5$ & 2 & -. & $\mathbf{s}$ & - & 09-23-82 & 97 & 6.8 & 7.0 & 05S15W 19DBAC01 \\
\hline 4.42 & 3 & 1.4 & $\mathbf{s}$ & - & $09-20-82$ & 368 & 7.2 & 10.0 & 05\$15W25BCCB01 \\
\hline 8.90 & 7 & 1.4 & D & D & -- & -. & -- & -- & 05S15W36CABD01 \\
\hline
\end{tabular}


Table 1. Records of selected wells by basin, Northern Rocky Mountains intermontane basins, Montana (Continued)

\begin{tabular}{|c|c|c|c|c|c|c|c|c|c|}
\hline Woll number & $\begin{array}{l}\text { Altitude } \\
\text { of land } \\
\text { surface } \\
\text { (ft) }\end{array}$ & $\begin{array}{l}\text { Date well } \\
\text { constructed }\end{array}$ & $\begin{array}{c}\text { Geologic } \\
\text { unit }\end{array}$ & $\begin{array}{l}\text { Depth } \\
\text { of } \\
\text { woll } \\
\text { (ft) }\end{array}$ & $\begin{array}{l}\text { Diam- } \\
\text { oter } \\
\text { of } \\
\text { casing } \\
\text { (in.) }\end{array}$ & $\begin{array}{l}\text { Type } \\
\text { of } \\
\text { finish }\end{array}$ & $\begin{array}{l}\text { Top of } \\
\text { open } \\
\text { inter- } \\
\text { val } \\
\text { (ft) }\end{array}$ & $\begin{array}{c}\text { Primary } \\
\text { use of } \\
\text { water }\end{array}$ & $\begin{array}{c}\text { Date water } \\
\text { level } \\
\text { measured }\end{array}$ \\
\hline \multicolumn{10}{|c|}{ Big Hole Basin--Continued } \\
\hline 06S15W09AADA01 & 6,578 & $07-25-79$ & Ts & 130 & 6.0 & $\mathbf{P}$ & 95 & $\mathbf{H}$ & $07-16-91$ \\
\hline 06S15W28BBAB01 & 6,764 & $06-30-79$ & Ts & 160 & 6.0 & $\mathbf{P}$ & 130 & $\mathbf{H}$ & $07-16-91$ \\
\hline 06S14W17CBDD01 & 6,685 & $09-14-80$ & Ts & 59 & 6.0 & $\mathbf{P}$ & 51 & $\mathbf{H}$ & $07-16-91$ \\
\hline 06S14W17CCAB01 & 6,690 & $07-17-80$ & Ts & 100 & 6.0 & $\mathbf{P}$ & 72 & $\mathbf{H}$ & $07-16-91$ \\
\hline \multicolumn{10}{|c|}{ Bitterroot Valley } \\
\hline 13N20W 26DDCD01 & 3,127 & $-\quad-78$ & Qal & 70 & 6.0 & - & - & $\mathbf{H}$ & $07-24-91$ \\
\hline $13 \mathrm{~N} 20 \mathrm{~W} 27 \mathrm{CC} \quad 0 \mathrm{I}$ & 3,275 & $04-21-88$ & Yms & 388 & 6.0 & $\mathbf{P}$ & 188 & $\mathbf{H}$ & $07-23-91$ \\
\hline 13N20W34BBBA01 & 3,250 & $05-12-91$ & Qal & 196 & 6.0 & $\mathbf{S}$ & 191 & $\mathbf{H}$ & $07-25-91$ \\
\hline 13N19W31BAC 01 & 3,155 & $12-11-74$ & Qal & 117 & 12.0 & $\mathbf{P}$ & 95 & $\mathbf{I}$ & $07-13-78$ \\
\hline 13N19W31BDAC01 & 3,155 & $04-11-75$ & Qal & 109 & 6.0 & $\mathbf{P}$ & -- & - & $07-23-91$ \\
\hline 12N20W03DCCD01 & 3,385 & $07-30-85$ & Yms & 300 & 6.0 & $\mathbf{P}$ & 240 & $\mathbf{H}$ & $08-02-91$ \\
\hline 12N20W10BAAB01 & 3,395 & $06-20-78$ & Yms & 260 & 6.0 & $\mathbf{P}$ & 150 & $\mathbf{H}$ & $07-24-91$ \\
\hline $12 \mathrm{~N} 20 \mathrm{~W} 11 \mathrm{BACCO}$ & 3,170 & $11-19-80$ & Qal & 81 & 6.0 & $\mathbf{O}$ & -- & $\mathbf{H}$ & $07-23-91$ \\
\hline 12N20W22ADACO1 & 3,140 & $06-17-92$ & Qal & 53 & 4.0 & $\mathbf{S}$ & 13 & $\mathbf{U}$ & $06-19-92$ \\
\hline 12N20W 22ADAC02 & 3,140 & $06-18-92$ & Qal & 48 & 4.0 & $\mathbf{S}$ & 8 & $\mathbf{U}$ & $06-19-92$ \\
\hline 12N20W 22ADAC03 & 3,140 & $06-18-92$ & Qal & 52 & 4.0 & $\mathbf{S}$ & 12 & $\mathbf{U}$ & $06-20-92$ \\
\hline 12N20W22ADAC04 & 3,140 & $06-20-92$ & Qal & 46 & 4.0 & $\mathbf{S}$ & 6 & $\mathbf{U}$ & 07-08-92 \\
\hline 12N20W25ACCA01 & 3,280 & $09-19-71$ & Qal & 70 & 6.0 & $\mathbf{O}$ & -- & $\mathbf{H}$ & - \\
\hline 12N20W 25ACCA02 & 3,280 & $07-18-88$ & Yms & 308 & 6.0 & $\mathbf{P}$ & 268 & $\mathbf{D}$ & $07-25-91$ \\
\hline 12N20W25CAAB01 & 3,180 & $06-30-76$ & Qal & 59 & 6.0 & $\mathbf{O}$ & -- & $\mathbf{S}$ & $07-24-91$ \\
\hline 12N20W 26CACCOI & 3,180 & $06-10-87$ & Yms & 103 & 6.0 & $\mathbf{P}$ & 43 & $\mathbf{P}$ & $07-25-91$ \\
\hline $12 \mathrm{~N} 20 \mathrm{~W} 34 \mathrm{CAB} \quad 01$ & 3,205 & -- & Qal & 62 & 6.0 & - & -- & $\mathbf{H}$ & $07-24-91$ \\
\hline $12 \mathrm{~N} 20 \mathrm{~W} 34 \mathrm{CBD} \quad 01$ & 3,235 & $04-07-83$ & Yms & 150 & 6.0 & $\mathbf{P}$ & 90 & $\mathbf{H}$ & $07-24-91$ \\
\hline 12N19W06ACCC01 & 3,230 & --61 & Ts & 283 & 7.0 & $\mathbf{S}$ & 273 & $\mathbf{U}$ & $07-23-91$ \\
\hline 12N19W06АCCCO2 & 3,240 & $09-05-61$ & Qal & 134 & 7.0 & $\mathbf{S}$ & 128 & $\mathbf{U}$ & $07-23-91$ \\
\hline 12N19W06BDBA01 & 3,166 & $06-22-66$ & Qal & 119 & 12.0 & $\mathbf{P}$ & 101 & $\mathbf{P}$ & $07-12-91$ \\
\hline $11 \mathrm{~N} 20 \mathrm{~W} 27 \mathrm{DDBB} 01$ & 3,355 & $12-04-89$ & Ts & 78 & 6.0 & $\mathbf{P}$ & 70 & $\mathbf{H}$ & $07-25-91$ \\
\hline 10N20W 10ACBCO1 & 3,540 & $09-21-77$ & Ts & 193 & 6.0 & $\mathbf{O}$ & -- & $\mathbf{H}$ & $07-26-91$ \\
\hline $10 \mathrm{~N} 20 \mathrm{~W} 13 \mathrm{BBA} 01$ & 3,204 & $05-\quad-59$ & Qal & 51 & 6.0 & $\mathbf{O}$ & - & $\mathbf{U}$ & $10-03-91$ \\
\hline 10N19W17DBCA01 & 3,500 & $09-14-78$ & Ts & 215 & 6.0 & $\mathbf{O}$ & -- & $\mathbf{H}$ & $07-25-91$ \\
\hline 10N19W18BADB01 & 3,270 & $01-03-58$ & Ts & 150 & 5.0 & $\mathbf{O}$ & -- & $\mathbf{H}$ & $07-24-91$ \\
\hline $09 \mathrm{~N} 20 \mathrm{~W} 11 \mathrm{ABCC} 01$ & 3,260 & $09-20-78$ & Qal & 52 & 6.0 & 0 & - & D & $07-27-91$ \\
\hline 09N20W20CDCD01 & 3,500 & $08-08-87$ & Ts & 160 & 6.0 & $\mathbf{P}$ & 60 & $\mathbf{H}$ & $07-27-91$ \\
\hline 09N20W26BACCO1 & 3,363 & $10-06-47$ & Ts & 380 & 10.0 & - & -- & $\mathbf{U}$ & $07-26-91$ \\
\hline 09N20W26BACC02 & 3,363 & -- & Ts & 552 & -- & - & -- & $\mathbf{U}$ & $07-26-91$ \\
\hline
\end{tabular}


Table 1. Records of selected wells by basin, Northern Rocky Mountains intermontane basins, Montana (Continued)

\begin{tabular}{|c|c|c|c|c|c|c|c|c|c|}
\hline $\begin{array}{c}\text { Water level } \\
\text { (ft) }\end{array}$ & $\begin{array}{c}\text { Dis- } \\
\text { charge } \\
\text { (gall } \\
\text { min) }\end{array}$ & $\begin{array}{c}\text { Specific } \\
\text { capacity } \\
\text { [(gal/ } \\
\text { min)/ft] }\end{array}$ & $\begin{array}{l}\text { Source } \\
\text { of dis- } \\
\text { charge } \\
\text { data }\end{array}$ & $\begin{array}{l}\text { Type } \\
\text { of log } \\
\text { avall- } \\
\text { able }\end{array}$ & $\begin{array}{c}\text { Date } \\
\text { water- } \\
\text { quallty } \\
\text { parameter } \\
\text { measured }\end{array}$ & $\begin{array}{c}\text { Specific } \\
\text { conduc- } \\
\text { tance } \\
(\mu \mathrm{S} / \mathrm{cm})\end{array}$ & $\begin{array}{l}\text { pH } \\
\text { (otan- } \\
\text { dard } \\
\text { units) }\end{array}$ & $\begin{array}{l}\text { Tem- } \\
\text { per- } \\
\text { ature } \\
\left({ }^{\circ} \mathrm{C}\right)\end{array}$ & Well number \\
\hline \multicolumn{10}{|c|}{ Big Hole Basin-Continued } \\
\hline 4.85 & 4 & 4.0 & $\mathbf{S}$ & $\mathrm{D}$ & $08-31-82$ & 88 & 6.6 & 6.0 & 06S15W09AADA0I \\
\hline 41.57 & 12 & 30 & $\mathbf{S}$ & D & $08-25-82$ & 139 & 7.0 & 8.0 & 06S15W28BBAB01 \\
\hline 2.71 & 11 & 1.8 & $\mathbf{S}$ & $\mathbf{D}$ & $08-30-82$ & 77 & 6.5 & 5.5 & 06S14W17CBDD01 \\
\hline 2.14 & 11 & .6 & $\mathbf{S}$ & D & $08-30-82$ & 126 & 7.3 & 6.0 & 06S14W 17CCAB01 \\
\hline \multicolumn{10}{|c|}{ Bitterroot Valley } \\
\hline 21.49 & 8 & 15 & $\mathbf{S}$ & - & $07-24-91$ & 385 & 7.2 & 11.0 & 13N20W26DDCD01 \\
\hline 163.19 & 9 & -- & D & $\mathrm{D}$ & -- & -- & -- & -- & $13 N 20 W 27 C C \quad 01$ \\
\hline 154.48 & 30 & 1.0 & D & $\mathrm{D}$ & $07-23-91$ & 280 & 7.1 & 8.5 & 13N20W34BBBA01 \\
\hline 25.55 & 1,200 & 200 & $\mathrm{D}$ & - & $07-25-78$ & -- & - & 9.5 & 13N19W3 IBAC 01 \\
\hline 27.31 & 220 & 14 & D & D & -- & -- & -- & -- & 13N19W3 IBDAC01 \\
\hline 122.63 & 20 & .3 & $\mathrm{D}$ & D & -- & -- & -- & -- & 12N20W03DCCD01 \\
\hline 108.80 & 25 & -- & $\mathrm{D}$ & D & $07-24-91$ & 474 & 7.4 & 12.0 & 12N20W 10BAAB01 \\
\hline 39.85 & 75 & 2.1 & $\mathrm{D}$ & D & $07-23-91$ & 209 & 7.3 & 10.0 & 12N20W $11 \mathrm{BACCO} 1$ \\
\hline 6.23 & -- & -- & - & $\mathrm{D}$ & -- & -- & $m$ & -- & 12N20W22ADAC0I \\
\hline 6.37 & -- & -- & - & $\mathbf{D}$ & $06-19-92$ & 145 & $\cdots$ & 10.5 & 12N20W22ADAC02 \\
\hline 4.92 & -- & -- & - & D & - & -- & - & -- & 12N20W22ADAC03 \\
\hline 7.13 & - & - & - & - & -- & -- & -- & $-\infty$ & 12N20W22ADAC04 \\
\hline-- & 10 & 1.3 & 0 & D & $07-31-78$ & -- & -- & 10.0 & 12N20W25ACCA01 \\
\hline 146.90 & 15 & .1 & $\mathbf{D}$ & D & -- & -- & - & -- & 12N20W25ACCA02 \\
\hline 18.52 & 50 & 1.5 & D & D & $07-24-91$ & 379 & 7.1 & 11.5 & 12N20W25CAAB0I \\
\hline 34.55 & 12 & .2 & $\mathrm{D}$ & D & -- & -- & -- & - & 12N20W26CACC01 \\
\hline 8.82 & 12 & 13 & $\mathbf{S}$ & - & $07-24-91$ & 144 & 7.1 & 9.0 & 12N20W34CAB 01 \\
\hline 39.41 & 20 & .3 & D & D & $07-24-91$ & 408 & 7.7 & 11.5 & 12N20W34CBD 01 \\
\hline 85.38 & 12 & -- & D & $\mathrm{D}$ & -- & - & -- & -- & 12N19W06ACCCOI \\
\hline 103.10 & -- & -- & - & $\mathrm{D}$ & - & - & -- & -- & 12N19W06ACCCO2 \\
\hline 35.65 & 1,500 & -- & $\mathrm{D}$ & D & -- & -- & -- & - & 12N19W06BDBA01 \\
\hline 48.82 & 1 & -- & $\mathrm{D}$ & D & -- & -- & -- & - & $11 \mathrm{~N} 20 \mathrm{~W} 27 \mathrm{DDBB} 01$ \\
\hline 40.21 & 20 & .1 & D & D & $07-25-91$ & 110 & 7.0 & 12.0 & $10 \mathrm{~N} 20 \mathrm{~W} 10 \mathrm{ACBC} 01$ \\
\hline 6.26 & - & -- & - & - & -- & -- & -- & -- & 10N20W 13BBA 01 \\
\hline 179.40 & 15 & .4 & D & $\mathbf{D}$ & $07-25-91$ & 199 & 7.1 & 12.0 & 10N19W17DBCA01 \\
\hline 36.40 & 9 & 3.7 & $\mathrm{D}$ & D & $07-24-91$ & 273 & 7.1 & 12.0 & 10N19W 18BADB01 \\
\hline 9.43 & 75 & 4.7 & D & $\mathbf{D}$ & $07-26-91$ & 325 & 7.3 & 13.0 & 09N20W11ABCCO1 \\
\hline 16.63 & 10 & .1 & D & D & $07-27-91$ & 242 & 8.0 & 10.0 & 09N20W20CDCDOI \\
\hline 81.65 & 400 & -- & D & $\mathrm{D}$ & -- & -- & -- & - & 09N20W26BACC01 \\
\hline 80.60 & -- & -- & - & - & -- & -- & - & -- & 09N20W26BACC02 \\
\hline
\end{tabular}


Table 1. Records of selected wells by basin, Northern Rocky Mountains intermontane basins, Montana (Continued)

\begin{tabular}{|c|c|c|c|c|c|c|c|c|c|}
\hline Woll number & $\begin{array}{l}\text { Altitude } \\
\text { of land } \\
\text { surface } \\
\text { (ft) }\end{array}$ & $\begin{array}{l}\text { Date well } \\
\text { constructed }\end{array}$ & $\begin{array}{c}\text { Geologic } \\
\text { unit }\end{array}$ & $\begin{array}{c}\text { Depth } \\
\text { of } \\
\text { well } \\
\text { (fi) }\end{array}$ & $\begin{array}{l}\text { Dlam- } \\
\text { otor } \\
\text { of } \\
\text { casing } \\
\text { (in.) }\end{array}$ & $\begin{array}{l}\text { Type } \\
\text { of } \\
\text { finlah }\end{array}$ & $\begin{array}{l}\text { Top of } \\
\text { open } \\
\text { Inter- } \\
\text { val } \\
\text { (ft) }\end{array}$ & $\begin{array}{l}\text { Primary } \\
\text { uso of } \\
\text { water }\end{array}$ & $\begin{array}{c}\text { Date water } \\
\text { lovel } \\
\text { measured }\end{array}$ \\
\hline \multicolumn{10}{|c|}{ Bitterroot Valley-Continued } \\
\hline 09N20W27AACC01 & 3,319 & $03-01-57$ & Ts & 455 & 10.0 & $\mathbf{P}$ & 362 & $\mathbf{P}$ & -- \\
\hline 09N20W27ABCCO1 & 3,305 & $09-13-74$ & Qal & 52 & 6.0 & $\mathbf{P}$ & 37 & I & $07-26-91$ \\
\hline 09N20W 27DBCD01 & 3,320 & $02-13-68$ & Qal & 56 & 8.0 & $\mathbf{P}$ & 36 & $\mathbf{P}$ & -- \\
\hline 09N20W27DCBA01 & 3,318 & $02-06-76$ & Qal & 75 & 8.0 & $\mathbf{P}$ & 40 & $\mathbf{P}$ & -. \\
\hline 09N20W27DCBB01 & 3,210 & $12-06-62$ & Qal & 54 & 6.0 & $\mathbf{P}$ & 37 & I & $07-26-91$ \\
\hline 09N20W27DDBA01 & 3,335 & $04-29-69$ & Qal & 100 & 8.0 & $\mathbf{P}$ & 80 & $\mathbf{U}$ & 03-29-69 \\
\hline 09N19W 14CBCC01 & 3,810 & $12-08-81$ & Ts & 192 & 6.0 & $\mathbf{P}$ & 120 & $\mathbf{H}$ & $07-26-91$ \\
\hline 09N19W 14CBCD01 & 3,815 & $06-18-82$ & Ts & 145 & 6.0 & $\mathbf{P}$ & 9 & $\mathbf{s}$ & 07-26-91 \\
\hline 08N21W24ACDA01 & 3,480 & $03-23-84$ & QTsu & 49 & 6.0 & $\mathbf{P}$ & 44 & $\mathbf{H}$ & $07-25-91$ \\
\hline 08N21W24BCBA01 & 3,640 & -- & QTsu & 92 & 6.0 & - & -- & $\mathbf{H}$ & $07-24-91$ \\
\hline 08N21W27DCB 01 & 3,650 & $05-24-78$ & Qal & 30 & 6.0 & $\mathbf{P}$ & 22 & $\mathbf{H}$ & 07-25-91 \\
\hline 08N20W01DAAD01 & 3,828 & $01-27-78$ & Ts & 132 & 6.0 & 0 & - & $\mathbf{H}$ & 07-26-91 \\
\hline 08N20W0IDABD01 & 3,822 & $08-10-77$ & Ts & 70 & 6.0 & 0 & - & $\mathbf{U}$ & $07-26-91$ \\
\hline 08N20W06CC 01 & 3,516 & -- & QTsu & 95 & 4.0 & - & - & $\mathbf{H}$ & $07-25-91$ \\
\hline 08N20W 16BADC01 & 3,320 & -- & Qal & 36 & 6.0 & $\cdot$ & - & $\mathbf{H}$ & $07-26-91$ \\
\hline 08N20W 19BAAD03 & 3,393 & $06-20-57$ & Ts & 52 & 6.0 & o & -- & $\mathrm{U}$ & $09-17-91$ \\
\hline 08N20W23CDDD01 & 3,545 & $07-20-84$ & Ts & 232 & 6.0 & 0 & -- & H & $07-26-91$ \\
\hline 08N20W28BACD01 & 3,348 & $05-28-86$ & Qal & 130 & 6.0 & $\mathbf{P}$ & 125 & HI & $07-25-91$ \\
\hline 08N20W28BDBA01 & 3,348 & $01-30-86$ & Qal & 68 & 6.0 & $\mathbf{P}$ & 60 & H & $07-25-91$ \\
\hline 08N20W31DDD 01 & 3,405 & $09-20-81$ & Qal & 59 & 6.0 & $\mathbf{P}$ & 51 & $\mathbf{H}$ & $07-25-91$ \\
\hline 08N19W07CBBD01 & 3,893 & -56 & Ts & 117 & 48.0 & o & -- & $\mathbf{H}$ & $09-17-91$ \\
\hline 08N19W1ICDBB01 & 4,230 & $12-21-79$ & Qal & 50 & 6.0 & $\mathbf{P}$ & 42 & $\mathbf{H}$ & $07-26-91$ \\
\hline O7N21W13BBAD01 & 3,580 & $06-13-79$ & QTsu & 100 & 6.0 & $\mathrm{o}$ & - & $\mathbf{H}$ & $07-24-91$ \\
\hline 07N21W22BBAC01 & 4,091 & $12-01-90$ & QTsu & 77 & 6.0 & $\mathbf{P}$ & 55 & - & 07-24-91 \\
\hline 07N21W22BBDB01 & 4,080 & $06-28-72$ & QTsu & 66 & 6.0 & $\mathbf{P}$ & 46 & $\mathbf{H}$ & $07-24-91$ \\
\hline 07N21W25CAB 01 & 3,494 & $10-17-87$ & Qal & 48 & 6.0 & $\mathbf{P}$ & 35 & $\mathbf{H}$ & $07-24-91$ \\
\hline O7N20W02BDDA01 & 3,550 & $02-26-79$ & Ts & 94 & 6.0 & $\mathbf{P}$ & 86 & $\mathbf{H}$ & $07-25-91$ \\
\hline 07N20W IIDADD01 & 3,690 & $11-14-90$ & QTsu & 85 & 6.0 & $\mathbf{P}$ & 41 & $\mathbf{H}$ & $07-24-91$ \\
\hline 07N20W23DCBB01 & 3,710 & $07-12-80$ & QTsu & 78 & 6.0 & $\mathbf{P}$ & 68 & $\mathbf{S}$ & $07-24-91$ \\
\hline 07N20W32DD 01 & 3,471 & $-\quad-58$ & Qal & 13 & 48.0 & $\mathrm{O}$ & -- & $\mathbf{F}$ & $07-23-91$ \\
\hline 07N20W32DDD 02 & 3,473 & --62 & Qal & 40 & 8.0 & O & - & $\mathbf{F}$ & $07-23-91$ \\
\hline 06N21 W 15CDDD01 & 3,918 & $10-01-79$ & QTsu & 120 & 6.0 & $\mathbf{P}$ & 70 & $\mathbf{H}$ & $07-17-91$ \\
\hline 06N21W25DBAA01 & 3,570 & $04-10-46$ & Qal & 66 & 12.0 & - & - & $\mathbf{P}$ & -- \\
\hline 06N21 W26DBAD01 & 3,613 & $01-15-81$ & QTsu & 79 & 6.0 & $\mathbf{P}$ & 74 & I & $07-16-91$ \\
\hline 06N21W35ABAB01 & 3,610 & $09-12-89$ & QTsu & 180 & 6.0 & $\mathbf{P}$ & 134 & $\mathbf{H}$ & $07-16-91$ \\
\hline
\end{tabular}


Table 1. Records of selected wells by basin, Northern Rocky Mountains intermontane basins, Montana (Continued)

\begin{tabular}{|c|c|c|c|c|c|c|c|c|c|}
\hline $\begin{array}{c}\text { Water lovel } \\
\text { (ft) }\end{array}$ & $\begin{array}{c}\text { Dis- } \\
\text { charga } \\
\text { (gall/ } \\
\text { min) }\end{array}$ & $\begin{array}{c}\text { Speciflc } \\
\text { capacity } \\
\text { [(gal/ } \\
\mathrm{mln}) / f t]\end{array}$ & $\begin{array}{l}\text { Source } \\
\text { of dis- } \\
\text { charge } \\
\text { data }\end{array}$ & $\begin{array}{l}\text { Type } \\
\text { of log } \\
\text { avall- } \\
\text { able }\end{array}$ & $\begin{array}{c}\text { Date } \\
\text { water- } \\
\text { quality } \\
\text { parameter } \\
\text { measured }\end{array}$ & $\begin{array}{c}\text { Specific } \\
\text { conduc- } \\
\text { tence } \\
(\mu \mathrm{S} / \mathrm{cm})\end{array}$ & $\begin{array}{c}\text { pH } \\
\text { (atan- } \\
\text { dard } \\
\text { units) }\end{array}$ & $\begin{array}{l}\text { Tem- } \\
\text { per- } \\
\text { ature } \\
\left.\text { ( }{ }^{\circ} \mathrm{C}\right)\end{array}$ & Well number \\
\hline
\end{tabular}

\begin{tabular}{|c|c|c|c|c|c|c|c|c|c|}
\hline .. & 400 & 5.7 & D & D & -- & -- & -- & -- & 09N20W27AACC01 \\
\hline 13.12 & 70 & 3.3 & D & D & -- & -- & -. & -- & 09N20W27ABCC01 \\
\hline -- & 100 & 17 & D & D & - & -. & - & -- & 09N20W27DBCD01 \\
\hline -- & 70 & 47 & D & D & -- & -. & -- & -. & 09N20W27DCBA01 \\
\hline 14.70 & 100 & 20 & D & D & -- & -- & - & -. & 09N20W27DCBB01 \\
\hline 38.00 & 50 & 2.3 & D & D & - & -- & -- & -- & 09N20W27DDBA01 \\
\hline 89.40 & 4 & .1 & D & D & $07-26-91$ & 288 & 7.3 & 12.5 & 09N19W14CBCC01 \\
\hline 71.59 & 6 & .1 & D & D & -- & -- & - & -- & 09N19W14CBCD01 \\
\hline 7.55 & 40 & 1.5 & D & D & -- & - & - & -- & 08N21W24ACDA01 \\
\hline 43.66 & -- & -- & - & D & 07-24-91 & 94 & 7.9 & 10.0 & 08N21W24BCBA01 \\
\hline 2.06 & 17 & 2.9 & $\mathbf{s}$ & D & $07-25-91$ & 71 & 6.6 & 10.5 & 08N21W27DCB 01 \\
\hline 52.32 & 30 & 7.9 & $\mathbf{s}$ & D & $07-26-91$ & 598 & 7.7 & 10.0 & 08N20W01DAAD01 \\
\hline 64.40 & 5 & - & D & D & -- & - & -- & -- & 08N20W01DABD01 \\
\hline 1.23 & 8 & 1.0 & $\mathbf{s}$ & - & $07-25-91$ & 147 & 6.9 & 12.0 & 08N20W06CC 01 \\
\hline 6.14 & -- & - & - & - & $07-26-91$ & 164 & 6.8 & 10.5 & 08N20W16BADC01 \\
\hline 14.34 & -- & $\cdots$ & - & D & - & -- & -- & -- & 08N20W 19BAAD03 \\
\hline 198.36 & 10 & .3 & D & D & -- & -- & - & -- & 08N20W 23CDDD01 \\
\hline 5.25 & 30 & .6 & D & D & $07-25-91$ & 240 & 6.8 & 12.0 & 08N20W28BACD01 \\
\hline 6.67 & 50 & 1.1 & D & D & - & -- & -- & $-\cdot$ & 08N20W28BDBA01 \\
\hline 37.30 & 6 & 3.2 & $\mathbf{S}$ & D & $07-25-91$ & 93 & 6.9 & 11.0 & 08N20W31DDD 01 \\
\hline 99.30 & -- & -- & - & - & -- & -- & -- & -- & 08N19W07CBBD01 \\
\hline 36.22 & 12 & 3.1 & $\mathbf{s}$ & D & - & -- & -. & - & 08N19WIICDBB0। \\
\hline 55.27 & 8 & .6 & $\mathbf{s}$ & D & $07-24-91$ & 102 & 7.3 & 11.0 & O7N21W13BBAD01 \\
\hline 48.88 & 5 & .3 & D & D & -- & - & -- & -- & 07N21W22BBAC01 \\
\hline 39.25 & 5 & .3 & D & D & -- & - & -- & -- & 07N21W22BBDB01 \\
\hline 12.30 & 10 & .8 & $\mathbf{s}$ & D & $07-24-91$ & 84 & 7.0 & 10.5 & $07 \mathrm{~N} 21 \mathrm{~W} 25 \mathrm{CAB} 01$ \\
\hline 4.07 & 10 & -. & D & D & $07-25-91$ & 393 & 7.4 & 12.5 & O7N20W02BDDA01 \\
\hline 31.42 & 8 & .2 & D & D & -- & -. & -- & -- & O7N20W11DADD01 \\
\hline 14.11 & 12 & .3 & D & D & $06-22-84$ & 502 & 7.7 & 11.5 & 07N20W23DCBB01 \\
\hline 7.08 & -. & - & - & - & -- & - & -- & - & 07N20W32DD 01 \\
\hline 7.03 & -- & -- & - & - & -- & - & -- & - & $07 \mathrm{~N} 20 \mathrm{~W} 32 \mathrm{DDD} 02$ \\
\hline 46.65 & 9 & 2.0 & $\mathbf{S}$ & D & $07-17-91$ & 53 & 6.7 & 10.0 & 06N21W15CDDD01 \\
\hline -- & 480 & -- & $\mathbf{R}$ & - & $08-17-92$ & 284 & 6.9 & 12.5 & 06N2 IW25DBAA01 \\
\hline 31.57 & 10 & .2 & D & D & - & -- & - & - & 06N21W26DBAD01 \\
\hline 90.52 & 10 & .6 & $\mathbf{s}$ & D & $07-16-91$ & 119 & 7.1 & 12.5 & 06N21W35ABAB01 \\
\hline
\end{tabular}


Table 1. Records of selected wells by basin, Northern Rocky Mountains intermontane basins, Montana (Continued)

\begin{tabular}{|c|c|c|c|c|c|c|c|c|c|}
\hline Well number & $\begin{array}{l}\text { Aittude } \\
\text { of land } \\
\text { surface } \\
\text { (ft) }\end{array}$ & $\begin{array}{l}\text { Date well } \\
\text { constructed }\end{array}$ & $\begin{array}{c}\text { Geologle } \\
\text { unit }\end{array}$ & $\begin{array}{c}\text { Depth } \\
\text { of } \\
\text { well } \\
\text { (fi) }\end{array}$ & $\begin{array}{c}\text { Dlam- } \\
\text { oter } \\
\text { of } \\
\text { caelng } \\
\text { (in.) }\end{array}$ & $\begin{array}{c}\text { Type } \\
\text { of } \\
\text { finish }\end{array}$ & $\begin{array}{l}\text { Top of } \\
\text { open } \\
\text { inter- } \\
\text { val } \\
\text { (ft) }\end{array}$ & $\begin{array}{l}\text { Primary } \\
\text { use of } \\
\text { water }\end{array}$ & $\begin{array}{c}\text { Date wate } \\
\text { level } \\
\text { measured }\end{array}$ \\
\hline \multicolumn{10}{|c|}{ Bitterroot Valley_Continued } \\
\hline 06N21W35BABD01 & 3,664 & $08-15-89$ & QTsu & 100 & 6.0 & $\mathbf{P}$ & 75 & H & $07-16-91$ \\
\hline 06N20W14BBBB01 & 3,792 & $04-06-79$ & QTsu & 1,110 & 4.0 & $\mathbf{P}$ & 209 & $\mathbf{U}$ & $07-17-91$ \\
\hline 06N20W15ADAD01 & 3,772 & $05-14-76$ & Ts & 80 & 6.0 & o & -- & $\mathbf{H}$ & $07-16-91$ \\
\hline 06N20W19CC 01 & 3,559 &.-56 & Qal & 20 & .8 & $T$ & -- & I & $07-16-91$ \\
\hline 06N20W $19 \mathrm{CCCCO} 2$ & 3,560 & 09-04-70 & Qal & 40 & 6.0 & o & - & $\mathrm{U}$ & $08-07-91$ \\
\hline 06N20W30DBCC01 & 3,581 & $06-18-75$ & QTsu & 109 & 12.0 & $\mathbf{p}$ & 87 & $\mathbf{P}$ & 07-16-91 \\
\hline 05N2IW02AAAB01 & 3,605 & $08-06-84$ & Qal & 52 & 6.0 & $\mathbf{p}$ & 44 & H & $07-15-91$ \\
\hline 05N21W15AABD01 & 3,922 & 09-08-72 & Ts & 301 & 8.0 & $\mathbf{P}$ & 220 & $\mathbf{H}$ & $07-15-91$ \\
\hline 05N21W15AADB0I & 3,915 & $10-19-88$ & QTsu & 60 & 6.0 & $\mathbf{P}$ & 18 & $\mathbf{s}$ & $07-15-91$ \\
\hline 05N21W15AADC0I & 3,910 & -- & Ts & 25 & -- & - & - & $\mathbf{S}$ & -- \\
\hline 05N21W15BDB 01 & 4,085 & -- & QTsu & 74 & 6.0 & - & -- & $\mathbf{H}$ & $07-15-91$ \\
\hline 05N21W34CBBB01 & 4,120 & $01-30-90$ & QTsu & 180 & 6.0 & $\mathbf{P}$ & 60 & $\mathbf{H}$ & $07-15-91$ \\
\hline $05 \mathrm{~N} 21 \mathrm{~W} 34 \mathrm{CCAD} 01$ & 3,998 & $08-26-71$ & Ts & 58 & 6.0 & - & - & $\mathbf{H}$ & $07-\mid 4-91$ \\
\hline 05N21W36DCC 01 & 3,700 & $06-27-90$ & Qal & 34 & 6.0 & $\mathbf{p}$ & 14 & $\mathbf{H}$ & $07-14-91$ \\
\hline 05N2IW36DCC 02 & 3,702 & $06-28-85$ & Qal & 29 & 8.0 & $\mathbf{P}$ & 14 & I & $07-14-91$ \\
\hline 05N20W04ACD 01 & 3,880 & $-\quad-79$ & Ts & 233 & 6.0 & $\mathbf{p}$ & 58 & $\mathbf{H}$ & $07-17-91$ \\
\hline 05N20W 10BAAC01 & 3,998 & 08-23-77 & QTsu & 80 & 6.0 & o & - & $\mathbf{s}$ & $07-17-91$ \\
\hline $05 \mathrm{~N} 20 \mathrm{~W} 18 \mathrm{CACC} 01$ & 3,685 & $02--64$ & QTsu & 75 & 6.0 & - & -- & $\mathbf{s}$ & $07-15-91$ \\
\hline 05N20W 18CBCD01 & 3,669 & $04-01-86$ & QTsu & 240 & 10.0 & $\mathbf{P}$ & 70 & $I$ & $07-15-91$ \\
\hline 05N20W 18CBDC01 & 3,670 & $05-08-86$ & QTsu & 120 & 10.0 & o & -- & $I$ & $07-15-91$ \\
\hline 05N20W 18CDDB01 & 3,780 & $03-16-79$ & QTsu & 295 & 6.0 & $\mathbf{p}$ & 235 & $\mathbf{H}$ & $07-15-91$ \\
\hline 05N20W 23BAC 01 & 3,990 & $07-18-80$ & TKi & 280 & 6.0 & $\mathbf{p}$ & 60 & $\mathbf{H}$ & $07-14-91$ \\
\hline $05 \mathrm{~N} 20 \mathrm{~W} 23 \mathrm{BBA}$ 01 & 3,975 & $04-18-80$ & Qal & 80 & 6.0 & $\mathbf{P}$ & 40 & $\mathbf{S}$ & $07-14-91$ \\
\hline 05N20W33BCCD01 & 3,850 & $09-19-85$ & Qal & 29 & 6.0 & $\mathbf{P}$ & 24 & H & $07-13-91$ \\
\hline 04N21W01CBCA01 & 3,820 & $06-04-90$ & TKi & 303 & 6.0 & o & - & H & $07-13-91$ \\
\hline 04N21 W 14CBAD01 & 3,795 & $01-18-89$ & Qal & 56 & 6.0 & $\mathbf{p}$ & 48 & H & $07-12-91$ \\
\hline 04N21 W16DDDD01 & 4,095 & -- & QTsu & 165 & 6.0 & - & - & $\mathbf{H}$ & $07-12-91$ \\
\hline 04N21W17BCBB01 & 4,154 & $12-24-79$ & QTsu & 160 & 6.0 & $\mathbf{p}$ & 136 & $\mathbf{P}$ & 07-13-91 \\
\hline 04N21 W28DDDD01 & 4,180 & -- & QTsu & 220 & 6.0 & - & - & H & $07-12-91$ \\
\hline 04N21W30DCDD01 & 4,318 & $02-02-62$ & QTsu & 95 & 6.0 & - & - & $\mathbf{P}$ & $07-13-91$ \\
\hline 04N21 W32BDAA01 & 4,252 & 06-04-79 & QTsu & 92 & 6.0 & o & -- & $\mathbf{P}$ & 07-13-91 \\
\hline 04N21 W33AADA01 & 4,195 & $08-10-82$ & QTsu & 160 & 6.0 & o & - & $\mathbf{H}$ & $07-12-91$ \\
\hline $04 \mathrm{~N} 21 \mathrm{~W} 35 \mathrm{CCBA01}$ & 3,844 & -- & Qal & 100 & 6.0 & - & -- & $\mathbf{I}$ & $07-12-91$ \\
\hline 03N21 W IODADDOI & 3,888 & $09-03-73$ & Qal & 70 & 10.0 & $\mathbf{p}$ & 40 & $\mathbf{P}$ & $07-12-91$ \\
\hline 03N21 W14BBDA01 & 3,890 & -. & Qal & -- & - & - & -- & $\mathbf{p}$ & - \\
\hline
\end{tabular}


Table 1. Records of selected wells by basin, Northern Rocky Mountains intermontane basins, Montana (Continued)

\begin{tabular}{|c|c|c|c|c|c|c|c|c|c|}
\hline $\begin{array}{c}\text { Water level } \\
\text { (ft) }\end{array}$ & $\begin{array}{c}\text { Dis- } \\
\text { charge } \\
\text { (gal/ } \\
\text { min) }\end{array}$ & $\begin{array}{c}\text { Specific } \\
\text { capecity } \\
\text { [(gal/ } \\
\text { min)/ft] }\end{array}$ & $\begin{array}{c}\text { Source } \\
\text { of dis- } \\
\text { charge } \\
\text { data }\end{array}$ & $\begin{array}{l}\text { Type } \\
\text { of log } \\
\text { avall- } \\
\text { able }\end{array}$ & $\begin{array}{c}\text { Date } \\
\text { water- } \\
\text { quality } \\
\text { parameter } \\
\text { measured }\end{array}$ & $\begin{array}{c}\text { Speclfic } \\
\text { conduc- } \\
\text { tance } \\
(\mu \mathrm{S} / \mathrm{cm})\end{array}$ & $\begin{array}{l}\text { pH } \\
\text { (stan- } \\
\text { dard } \\
\text { units) }\end{array}$ & $\begin{array}{l}\text { Tem- } \\
\text { per- } \\
\text { ature } \\
\left({ }^{\circ} \mathrm{C}\right)\end{array}$ & Well number \\
\hline
\end{tabular}

\begin{tabular}{|c|c|c|c|c|c|c|c|c|c|}
\hline 19.96 & 50 & .9 & D & $\mathbf{D}$ & -- & -- & -- & -. & 06N21W35BABD01 \\
\hline 67.22 & 32 & .7 & $\mathrm{D}$ & D & -- & _- & .- & -- & 06 N20W $14 \mathrm{BBBB} 01$ \\
\hline 43.28 & 25 & 1.4 & D & D & -- & -- & -- & -. & 06N20W15ADAD01 \\
\hline 7.39 & -- & $\cdots$ & - & - & $m$ & -- & -- & -- & $06 \mathrm{~N} 20 \mathrm{~W} 19 \mathrm{CC} \quad 01$ \\
\hline 6.51 & -- & -- & - & - & -- & -- & - & $-\cdot$ & $06 \mathrm{~N} 20 \mathrm{~W} 19 \mathrm{CCCC} 02$ \\
\hline 4.97 & 500 & 6.9 & D & D & -- & -- & -- & -- & $06 \mathrm{~N} 20 \mathrm{~W} 30 \mathrm{DBCC} 01$ \\
\hline 23.00 & 40 & 2.7 & D & D & -- & -. & -- & -- & 05N21W02AAAB01 \\
\hline 156.96 & 16 & .1 & D & D & $07-15-91$ & 164 & 7.7 & 13.5 & 05N21W15AABD01 \\
\hline 5.50 & 15 & .5 & D & D & -- & - & -- & -- & 05N21W15AADB01 \\
\hline-- & 5 & -- & $\mathbf{S}$ & - & $08-18-92$ & 36 & 6.1 & 10.5 & 05N21W 15AADC01 \\
\hline 48.22 & -- & -. & - & - & -- & -- & - & -- & $05 \mathrm{~N} 21 \mathrm{~W} 15 \mathrm{BDB} 01$ \\
\hline 27.72 & 2 & -- & D & D & -- & -- & -- & -- & 05N21W34CBBB01 \\
\hline 15.98 & 5 & .1 & $\mathbf{D}$ & D & -- & -- & -- & -- & $05 \mathrm{~N} 21 \mathrm{~W} 34 \mathrm{CCAD} 01$ \\
\hline 7.19 & 7 & 7.3 & $\mathbf{S}$ & D & $07-14-91$ & 90 & 6.6 & 11.0 & 05N21W36DCC 01 \\
\hline 7.08 & 99 & 9.0 & $\mathbf{D}$ & D & -- & -- & -- & -- & 05N21W36DCC 02 \\
\hline 103.24 & 35 & .4 & D & D & 07-17-91 & 616 & 7.8 & 14.0 & 05N20W04ACD 01 \\
\hline 22.75 & 20 & .7 & D & D & $07-17-91$ & 618 & 7.7 & 11.0 & 05N20W10BAAC01 \\
\hline 28.12 & - & -- & - & D & -- & -- & - & -- & 05N20W18CACC01 \\
\hline 15.97 & 900 & 9.5 & D & D & -- & -. & -- & -- & 05N20W18CBCD01 \\
\hline 18.20 & 300 & 3.5 & $\mathbf{D}$ & $\mathbf{D}$ & -- & - & - & $-\cdot$ & 05N20W $18 \mathrm{CBDC} 01$ \\
\hline 79.35 & 40 & .3 & D & D & $07-15-91$ & 361 & 7.5 & 14.0 & 05N20W $18 C D D B 01$ \\
\hline 24.26 & 2 & .2 & $\mathbf{S}$ & D & $07-14-91$ & 486 & 6.9 & 14.0 & 05N20W23BAC 01 \\
\hline 29.88 & 2 & .1 & D & D & -- & -- & -- &.- & 05N20W23BBA 01 \\
\hline 9.51 & 9 & 8.9 & $\mathbf{S}$ & D & $07-13-91$ & 209 & 6.8 & 11.0 & 05N20W33BCCD0 1 \\
\hline 20.02 & 10 & -- & D & $\mathbf{D}$ & - & -- & -- & -- & $04 \mathrm{~N} 21 \mathrm{~W} 01 \mathrm{CBCA} 01$ \\
\hline 30.17 & 24 & 39 & $\mathbf{S}$ & $\mathbf{D}$ & $07-12-91$ & 333 & 7.0 & 11.0 & 04N21W 14CBAD01 \\
\hline 38.75 & 8 & .4 & $\mathbf{s}$ & - & 07-12-91 & 70 & 6.7 & 11.5 & 04N21W16DDDD01 \\
\hline 122.73 & 10 & .3 & D & D & -- & -. & -- & -- & O4N21W17BCBB01 \\
\hline 200.43 & -. & -. & - & - & -- & -. & -- & -- & 04N21W28DDDD01 \\
\hline 63.67 & 6 & .2 & $\mathbf{D}$ & $\mathbf{D}$ & -- & -- & -- & -- & 04N2 1W30DCDD01 \\
\hline 24.40 & -- & -- & - & D & $07-13-91$ & 54 & 7.3 & 12.0 & 04N21W32BDAA01 \\
\hline 91.24 & 12 & .3 & D & - & -- & -- & -- & -- & 04N21W33AADA01 \\
\hline 10.92 & -- & -- & - & - & -- & -- & - & -- & 04N21W35CCBA01 \\
\hline 11.77 & 500 & 9.6 & D & D & -- & -- & -- & -- & 03N21W10DADD01 \\
\hline .. & -- & -- & - & - & $08-19-92$ & 159 & 6.4 & 10.5 & 03N21W14BBDA01 \\
\hline
\end{tabular}


Table 1. Records of selected wells by basin, Northern Rocky Mountains intermontane basins, Montana (Continued)

\begin{tabular}{|c|c|c|c|c|c|c|c|c|c|}
\hline Well number & $\begin{array}{l}\text { Altitude } \\
\text { of land } \\
\text { surface } \\
\text { (ft) }\end{array}$ & $\begin{array}{l}\text { Date well } \\
\text { constructed }\end{array}$ & $\begin{array}{c}\text { Goologle } \\
\text { unit }\end{array}$ & $\begin{array}{c}\text { Depth } \\
\text { of } \\
\text { well } \\
\text { (ft) }\end{array}$ & $\begin{array}{c}\text { Diam- } \\
\text { oter } \\
\text { of } \\
\text { casing } \\
\text { (ln.) } \\
\end{array}$ & $\begin{array}{c}\text { Type } \\
\text { of } \\
\text { finish }\end{array}$ & $\begin{array}{l}\text { Top of } \\
\text { open } \\
\text { Inter- } \\
\text { vel } \\
\text { (tt) }\end{array}$ & $\begin{array}{c}\text { Primary } \\
\text { use of } \\
\text { water }\end{array}$ & $\begin{array}{l}\text { Dato wat } \\
\text { lovel } \\
\text { mensure }\end{array}$ \\
\hline \multicolumn{10}{|c|}{ Bitterroot Valley-Continued } \\
\hline 03N21W14CAAA0I & 3,895 & $|1-12-8|$ & Qal & 80 & 8.0 & $\mathbf{P}$ & 61 & $\mathbf{P}$ & $07-12-91$ \\
\hline 03N21 W15CCCD01 & 4,140 & 08-08-76 & QTsu & 111 & 6.0 & $\mathbf{P}$ & 101 & H & $07-11-91$ \\
\hline $03 N 21$ W22BDA 01 & 4,040 & -- & QTsu & 93 & 6.0 & - & -- & $\mathbf{S}$ & $07-11-91$ \\
\hline 03N21W26BBB 01 & 4,100 & $09-21-74$ & QTsu & 84 & 6.0 & 0 & -- & $\mathbf{s}$ & $07-11-91$ \\
\hline 03N20W3IDBBC01 & 4,005 & -- & Qal & 40 & 6.0 & - & -- & $\mathbf{s}$ & $07-11-91$ \\
\hline 03N20W31DBCC01 & 3,985 & $05-14-76$ & Qal & 61 & 6.0 & o & -- & $\mathbf{H}$ & $07-1 \mid-91$ \\
\hline $02 \mathrm{~N} 21 \mathrm{~W} 12 \mathrm{CDBA01}$ & 4,180 & $05-31-84$ & QTsu & 300 & 6.0 & $\mathbf{P}$ & 10 & $\mathbf{H}$ & $07-11-91$ \\
\hline 02N2I W12DCAC01 & 4,075 & -- & QTsu & 84 & 6.0 & - & -- & $\mathbf{H}$ & $07-11-91$ \\
\hline 02N21W34CBD 01 & 4,182 & $07-29-86$ & Qal & 40 & 8.0 & $\mathbf{P}$ & 25 & I & 07-11-91 \\
\hline 02N2IW34CDAC01 & 4,170 & $06-22-73$ & QTsu & 36 & 6.0 & $\mathrm{O}$ & -- & $\mathbf{H}$ & $07-10-91$ \\
\hline 02N20W07BCCC01 & 4,055 & $05-03-78$ & Qal & 60 & 6.0 & o & -- & U & $07-11-91$ \\
\hline 02N20W22CBCA01 & 4,140 & - & Qal & 37 & 6.0 & - & -- & H & $07-11-91$ \\
\hline 01N20W12ABAB01 & 4,360 & $09-23-76$ & TKi & 200 & 6.0 & $\mathbf{P}$ & 120 & H & $07-10-91$ \\
\hline 01N20W I2CCCD01 & 4,438 & 07-07-83 & TKi & 85 & 6.0 & $\mathbf{P}$ & 50 & $\mathbf{H}$ & $07-10-91$ \\
\hline 0IN20W 12 CCDB0! & 4,435 & $11-18-82$ & Qal & 39 & 6.0 & $\mathbf{P}$ & 30 & $\mathrm{U}$ & $07-10-91$ \\
\hline 01N19W10CBBB01 & 4,488 & $04-\quad-86$ & QTsu & 56 & 6.0 & o & - & $\mathbf{P}$ & $07-10-91$ \\
\hline 01N19W 16DDAA01 & 4,446 & $\cdots$ & QTsu & 34 & 6.0 & $\cdot$ & - & $\mathbf{H}$ & $07-10-91$ \\
\hline \multicolumn{10}{|c|}{ Blackfoot-Clearwater Valley } \\
\hline 17N15W33BABA01 & 4,010 & $02-19-62$ & $\mathrm{Qg}$ & 153 & 8.0 & o & - & $\mathbf{P}$ & $09-23-91$ \\
\hline 17N15W34ACDC01 & 4,010 & $06-23-63$ & $\mathrm{Qg}$ & 663 & 8.0 & o & - & $\mathrm{U}$ & 09-24-91 \\
\hline 16N16W14DDCC01 & 4,250 & $-\quad-75$ & QTsu & 200 & 6.0 & - & .. & $\mathrm{U}$ & 09-24-91 \\
\hline 16N15W01BABA01 & 4,196 & $06-30-78$ & QTsu & 70 & 6.0 & $\mathrm{o}$ & -- & $\mathrm{U}$ & $09-23-91$ \\
\hline 16N15W01BADB01 & 4,183 & $09-28-73$ & QTsu & 80 & 6.0 & $\mathbf{P}$ & 75 & $\mathbf{H}$ & $09-24-91$ \\
\hline 16N14W25AADA01 & 4,314 & 08-24-89 & Qal & 98 & 6.0 & - & -- & $\mathbf{H}$ & 09-18-91 \\
\hline 16N14W25BCCB0I & 4,282 & $11-16-84$ & QTsu & 340 & 6.0 & $\mathbf{P}$ & 240 & $\mathbf{H}$ & $09-20-91$ \\
\hline $16 \mathrm{~N} 14 \mathrm{~W} 25 \mathrm{CDDC} 01$ & 4,242 & $08-03-89$ & Qal & 56 & 6.0 & 0 & -- & $\mathbf{H}$ & $09-20-91$ \\
\hline $16 \mathrm{~N} 12 \mathrm{~W} 20 \mathrm{CBBA0} 1$ & 4,195 & $10-10-86$ & QTsu & 36 & 6.0 & $\mathrm{o}$ & - & $\mathbf{P}$ & $09-12-91$ \\
\hline $16 \mathrm{~N} 12 \mathrm{~W} 30 \mathrm{AACC} 01$ & 4,135 & $-\quad-69$ & QTsu & 750 & 6.0 & - & - & $\mathbf{H}$ & $09-19-91$ \\
\hline 15N14W05ABAB0I & 3,925 & $07-30-79$ & Qal & 60 & 6.0 & $\mathrm{O}$ & -- & $\mathbf{P}$ & $09-24-91$ \\
\hline 15N14W09BBCA01 & 3,920 & $05-02-84$ & Qal & 60 & 6.0 & $\mathrm{O}$ & - & $\mathrm{U}$ & $09-24-91$ \\
\hline 15N14W25DBDD01 & 3,858 & $09-27-88$ & QTsu & 64 & 6.0 & $\mathrm{O}$ & -- & $\mathbf{P}$ & $09-24-91$ \\
\hline 15N14W36BCDB01 & 3,850 & $-\quad-72$ & Qal & 80 & 6.0 & $\mathrm{O}$ & -- & $\mathbf{H}$ & $09-19-91$ \\
\hline 15N13W12CACA01 & 4,090 & - & QTsu & 78 & 6.0 & - & - & $\mathbf{H}$ & $09-24-91$ \\
\hline 15N13W32ACCA01 & 3,905 & $06-23-82$ & Qal & 36 & 6.0 & $\mathbf{P}$ & 28 & C & $09-19-91$ \\
\hline 15N12W05CDCA01 & 4,125 & $-\quad-55$ & QTsu & 165 & 6.0 & - & - & $\mathbf{s}$ & $09-18-91$ \\
\hline 15N12W25CADD01 & 4,338 & $10-10-77$ & QTsu & 160 & 6.0 & $\mathbf{P}$ & 152 & $\mathbf{H}$ & $09-20-91$ \\
\hline 15N12W25CDAD01 & 4,340 & --71 & QTsu & 47 & 6.0 & 0 & - & $\mathbf{H}$ & $09-18-91$ \\
\hline 15N12W29BDBD01 & 4,075 & $-\quad-68$ & QTsu & 160 & 6.0 & - & - & $\mathbf{H}$ & $09-19-91$ \\
\hline
\end{tabular}


Table 1. Records of selected wells by basin, Northern Rocky Mountains intermontane basins, Montana (Continued)

\begin{tabular}{|c|c|c|c|c|c|c|c|c|c|}
\hline $\begin{array}{c}\text { Water level } \\
\text { (tt) }\end{array}$ & $\begin{array}{l}\text { Dle- } \\
\text { charge } \\
\text { (gall } \\
\text { min) }\end{array}$ & $\begin{array}{c}\text { Specific } \\
\text { capacity } \\
\text { [(gal/ } \\
\text { m/n)/ft] }\end{array}$ & $\begin{array}{l}\text { Source } \\
\text { of die- } \\
\text { charge } \\
\text { data }\end{array}$ & $\begin{array}{l}\text { Type } \\
\text { cf log } \\
\text { avell- } \\
\text { able }\end{array}$ & $\begin{array}{c}\text { Dale } \\
\text { water- } \\
\text { quality } \\
\text { paremeter } \\
\text { measured }\end{array}$ & $\begin{array}{c}\text { Specific } \\
\text { conduc- } \\
\text { tance } \\
(\mu \mathrm{S} / \mathrm{cm})\end{array}$ & $\begin{array}{l}\text { PH } \\
\text { (stan- } \\
\text { dard } \\
\text { unlts) }\end{array}$ & $\begin{array}{l}\text { Tem- } \\
\text { per- } \\
\text { ature } \\
\left({ }^{\circ} \mathrm{C}\right)\end{array}$ & Well number \\
\hline
\end{tabular}

Bitterroot Valley-Continued

\begin{tabular}{|c|c|c|c|c|c|c|c|c|c|}
\hline 7.53 & 250 & 5.0 & $\mathrm{D}$ & D & -- & -- & -- & $\cdots$ & 03N21W14CAAA01 \\
\hline 67.20 & 8 & .3 & D & D & -- & -- & -- & -- & 03N21W15CCCD01 \\
\hline 21.45 & -- & -- & - & - & - & -- & -- & -- & 03N21W22BDA 01 \\
\hline 41.85 & 15 & -- & D & D & - & - & -- & -- & 03N21W26BBB 01 \\
\hline 21.86 & -- & -- & - & - & -- & -- & -- & -- & $03 \mathrm{~N} 20 \mathrm{~W} 3 \mathrm{IDBBC} 01$ \\
\hline 12.05 & 60 & 1.5 & D & D & - & -- & -- & -- & $03 \mathrm{~N} 20 \mathrm{~W} 31 \mathrm{DBCC} 01$ \\
\hline 27.02 & 2 & -. & D & D & -- & -- & -- & -- & 02N21W12CDBA01 \\
\hline 14.17 & -- & -- & - & - & - & -- & -- & -- & 02N21W12DCAC01 \\
\hline 7.90 & 500 & 19 & D & D & -- & -- & -- & -- & 02N21W34CBD 01 \\
\hline 5.99 & 10 & 6.8 & $\mathbf{S}$ & D & $07-10-91$ & 97 & 7.0 & 7.5 & 02N21W34CDACO1 \\
\hline 29.48 & 20 & .9 & D & D & -- & -- & -- & -- & 02N20W07BCCC01 \\
\hline 11.25 & 12 & 3.4 & $\mathbf{S}$ & - & $07-11-91$ & 142 & 7.5 & 9.0 & 02N2OW22CBCA01 \\
\hline 70.70 & 5 & .1 & D & D & - & -- & -- & -- & 01N20W12ABAB01 \\
\hline 4.46 & 35 & .6 & D & D & -- & -- & -- & -- & OIN20W $12 C C C D 01$ \\
\hline 5.23 & 20 & 1.4 & D & D & -- & - & -- & -. & $01 \mathrm{~N} 20 \mathrm{~W} 12 \mathrm{CCDB} 01$ \\
\hline+.05 & 8 & 40 & $\mathbf{s}$ & D & 07-10-91 & 114 & 6.9 & 11.0 & $01 N 19 W 10 C B B B 01$ \\
\hline 4.08 & 7 & 3.3 & $\mathbf{S}$ & - & $07-10-91$ & 162 & 7.3 & 7.5 & 01N19W16DDAA01 \\
\hline
\end{tabular}

Blackfoot-Clearwater Valley

\begin{tabular}{|c|c|c|c|c|c|c|c|c|c|}
\hline 21.90 & 12 & .2 & D & D & -- & -- & -- & -- & 17N15W33BABA01 \\
\hline 1.35 & 17 & .1 & D & D & -- & - & -- & -- & 17N15W34ACDC01 \\
\hline 36.35 & -. & -. & - & - & -- & -- & -- & -. & 16N16W14DDCC01 \\
\hline 10.73 & 15 & .4 & D & D & -- & -- & - & -- & 16N15W01BABA01 \\
\hline 15.36 & 7 & .1 & D & - & $08-11-76$ & -- & 7.7 & 13.0 & 16N15W01BADB01 \\
\hline 79.97 & 15 & .8 & $\mathbf{D}$ & D & -- & -- & - & -- & 16N14W25AADA01 \\
\hline 2.73 & 15 & .1 & D & D & -. & -- & -- & -- & $16 \mathrm{~N} 14 \mathrm{~W} 25 \mathrm{BCCB} 1$ \\
\hline 28.34 & 50 & 1.9 & D & D & -- &.- & -- & -- & $16 \mathrm{~N} 14 \mathrm{~W} 25 \mathrm{CDDC} 01$ \\
\hline 6.54 & 30 & 1.1 & D & D & - & .- & -- & -- & $16 \mathrm{~N} 12 \mathrm{~W} 20 \mathrm{CBBA0} 1$ \\
\hline 85.04 & -- & -- & - & - & -- & - & - & -- & 16N12W30AACC01 \\
\hline 42.29 & 25 & 1.9 & D & D & -- & - & -- & -- & 15N14W05ABAB01 \\
\hline 11.92 & 50 & 3.6 & D & D & -- & - & - & $\cdots$ & 15N14W09BBCA01 \\
\hline 20.03 & 15 & 3.8 & D & D & -- & -- & -- & -- & 15N14W25DBDD01 \\
\hline 29.98 & -- & -- & - & - & -- & -- & -- & -. & $15 \mathrm{~N} 14 \mathrm{~W} 36 \mathrm{BCDB} 01$ \\
\hline 11.63 & -- & -- & - & - & 09-24-91 & 111 & -- & 11.5 & 15N13W12CACA01 \\
\hline 17.60 & 50 & 6.3 & D & D & -- & -- & -- & -- & $15 N 13 W 32 A C C A 01$ \\
\hline 30.96 & -- & -- & - & - & -- & -- & -- & -- & 15N12W05CDCA01 \\
\hline 52.12 & 12 & -- & D & D & -- & -- & -. & -- & 15N12W25CADD01 \\
\hline .67 & -- & -- & - & - & - & -- & -- & - & 15N12W25CDAD01 \\
\hline 12.57 & -- & -- & - & - & -- & -- & -. & -- & $15 N 12 W 29 B D B D O 1$ \\
\hline
\end{tabular}


Table 1. Records of selected wells by basin, Northern Rocky Mountains intermontane basins, Montana (Continued)

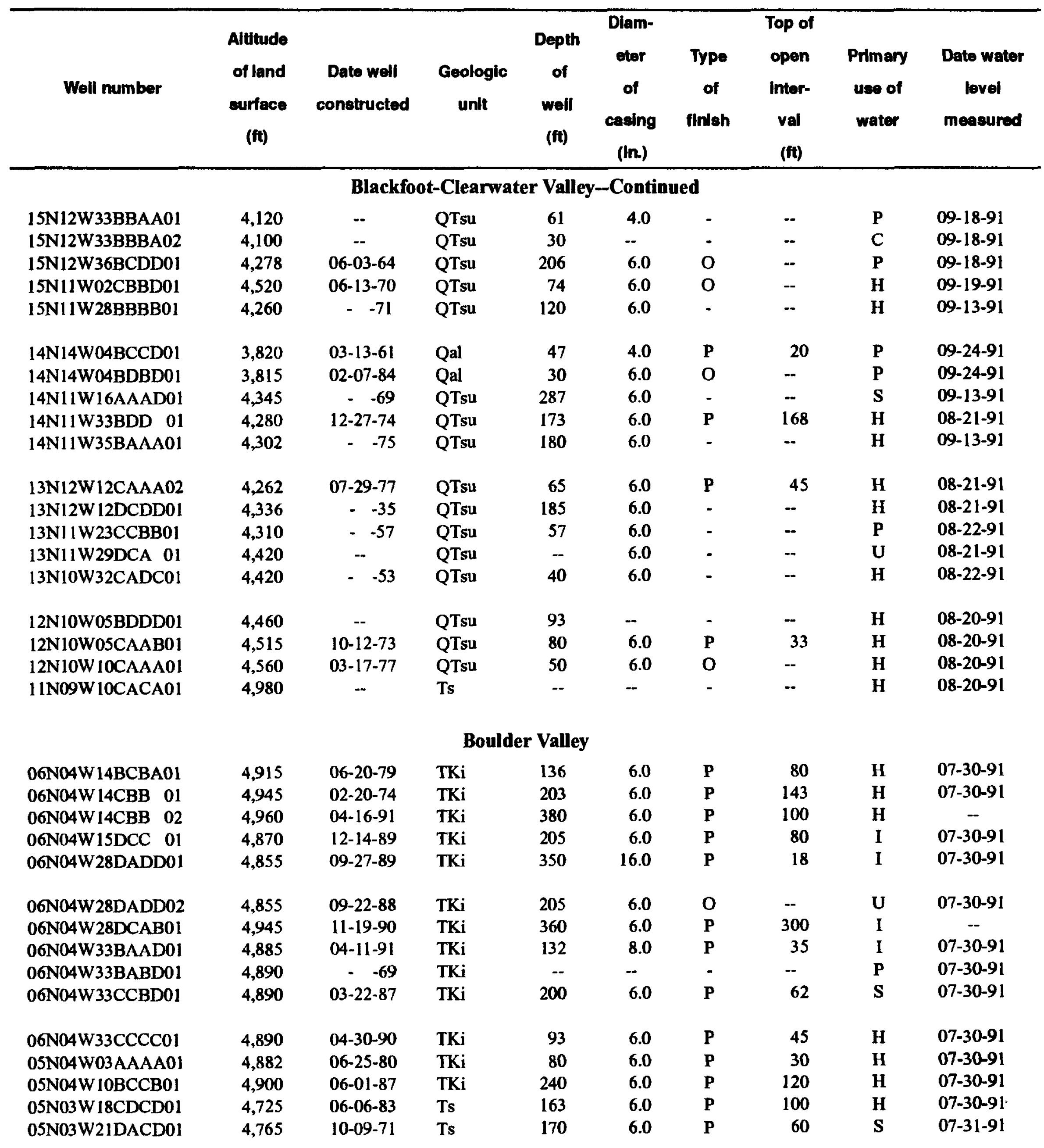


Table 1. Records of selected wells by basin, Northern Rocky Mountains intermontane basins, Montana (Continued)

\begin{tabular}{|c|c|c|c|c|c|c|c|c|c|}
\hline $\begin{array}{c}\text { Water level } \\
\text { (ti) }\end{array}$ & $\begin{array}{c}\text { Dis- } \\
\text { charge } \\
\text { (gal/ } \\
\text { min) }\end{array}$ & $\begin{array}{c}\text { Spocific } \\
\text { capacity } \\
\text { [(gal/ } \\
\text { min)/ft] }\end{array}$ & $\begin{array}{l}\text { Source } \\
\text { of dis- } \\
\text { charge } \\
\text { data }\end{array}$ & $\begin{array}{l}\text { Type } \\
\text { of log } \\
\text { evall- } \\
\text { able }\end{array}$ & $\begin{array}{c}\text { Date } \\
\text { water- } \\
\text { quallty } \\
\text { parameter } \\
\text { measured }\end{array}$ & $\begin{array}{l}\text { Specific } \\
\text { conduc- } \\
\text { tance } \\
(\mu S / \mathrm{cm})\end{array}$ & $\begin{array}{c}\text { pH } \\
\text { (atan- } \\
\text { dard } \\
\text { units) }\end{array}$ & $\begin{array}{l}\text { Tem- } \\
\text { per- } \\
\text { ature } \\
\left({ }^{\circ} \mathrm{C}\right)\end{array}$ & Well number \\
\hline
\end{tabular}

\begin{tabular}{|c|c|c|c|c|c|c|c|c|c|}
\hline 40.19 & -- & -- & - & - & -- & -- & -- & -- & $15 \mathrm{~N} 12 \mathrm{~W} 33 \mathrm{BBAA0} 1$ \\
\hline 11.09 & -- & -- & - & - & -- & -- & -- & -- & 15N12W33BBBA02 \\
\hline 96.24 & 35 & .3 & D & D & $08-13-76$ & -- & 7.4 & 11.5 & 15N12W36BCDD01 \\
\hline 54.65 & 15 & 1.7 & D & D & -- & -- & -- & -- & $15 \mathrm{~N} 11 \mathrm{~W} 02 \mathrm{CBBD} 01$ \\
\hline 36.26 & -- & -- & - & - & -- & -- & -- & - & $15 \mathrm{~N} 11 \mathrm{~W} 28 \mathrm{BBBB} 01$ \\
\hline 33.44 & 25 & -. & D & D & - & -- & -- & - & 14N14W04BCCD01 \\
\hline 22.13 & 10 & 2.6 & D & D & -- & -- & -- & -- & 14N14W04BDBD01 \\
\hline 44.80 & -- & -- & - & - & -- & -- & -- & -- & 14N11W16AAAD01 \\
\hline 8.93 & 25 & 4.2 & D & D & $09-24-75$ & -- & 7.8 & 9.0 & 14N11W33BDD 01 \\
\hline 12.17 & - & -- & - & - & -- & - & - & -- & 14N11W35BAAA01 \\
\hline 24.88 & 30 & 15 & D & D & -- & -- & -- & -- & 13N12W12CAAA02 \\
\hline 12.51 & -- & -- & - & - & -. & -. & -- & -- & 13N12W12DCDD01 \\
\hline .04 & $-\cdot$ & -- & - & - & $08-13-76$ & -- & 7.4 & 11.5 & 13N11W23CCBB01 \\
\hline 45.35 & -- & -- & - & - & -- & -- & -- & -- & 13N1IW29DCA 01 \\
\hline 15.85 & -- & -- & - & - & - & -- & -- & -- & 13N10W32CADC01 \\
\hline 27.43 & -- & -- & - & - & -- & -- & - & -- & 12N10W05BDDD01 \\
\hline 5.90 & 15 & -- & D & D & -- & - & - & -- & 12N10W05CAAB01 \\
\hline 19.48 & 10 & 1.7 & D & D & - & -- & -- & -- & 12N10W 10CAAA01 \\
\hline 6.73 & -- & -. & - & - & -- & -- & - & -- & IIN09W10CACA01 \\
\hline \multicolumn{10}{|c|}{ Boulder Valley } \\
\hline 11.87 & 10 & .6 & $\mathbf{S}$ & D & $07-30-91$ & 388 & 7.3 & 8.5 & O6N04W 14BCBA0I \\
\hline 17.97 & 8 & -- & D & D & -- & -- & -- & -- & 06N04W14CBB 01 \\
\hline-- & 5 & -- & D & D & $07-30-91$ & 294 & 7.7 & -- & 06N04W14CBB 02 \\
\hline 2.07 & 300 & 1.6 & D & D & -. & -. & -- & -- & 06N04W15DCC 01 \\
\hline 2.66 & 1,500 & 40 & D & D & - & -- & -- & - & 06N04W28DADD01 \\
\hline 1.74 & 100 & .5 & D & D & -- & - & -- & -- & 06N04W28DADD02 \\
\hline-- & 40 & -- & D & D & -- & - & -- & -. & 06N04W28DCAB01 \\
\hline 7.25 & 150 & 1.4 & D & D & -. & - & -- & -- & 06N04W33BAAD01 \\
\hline 9.90 & -- & -- & - & - & -- & - & -- & -- & 06N04W33BABD01 \\
\hline 10.35 & 15 & .1 & D & D & $07-30-91$ & 157 & 7.2 & 9.0 & 06N04W33CCBD01 \\
\hline 8.65 & 50 & .7 & D & D & 07-30-91 & 375 & 7.6 & 11.0 & 06N04W33CCCC01 \\
\hline 28.70 & 3 & -- & D & D & $07-30-91$ & 405 & 7.2 & 12.0 & 05N04W03AAAA01 \\
\hline 34.51 & 7 & -- & D & D & -- & - & -- & -- & 05N04W10BCCB01 \\
\hline 48.85 & 20 & .2 & D & D & $07-30-91$ & 449 & 7.7 & 14.0 & 05N03W18CDCD01 \\
\hline 10.45 & 8 & .1 & $\mathrm{D}$ & D & - & -- & -. & -- & 05N03W21DACD01 \\
\hline
\end{tabular}


Table 1. Records of selected wells by basin, Northern Rocky Mountains intermontane basins, Montana (Continued)

\begin{tabular}{|c|c|c|c|c|c|c|c|c|c|}
\hline Wall number & $\begin{array}{l}\text { Altituda } \\
\text { of land } \\
\text { surface } \\
\text { (ft) }\end{array}$ & $\begin{array}{l}\text { Date well } \\
\text { constructed }\end{array}$ & $\begin{array}{c}\text { Geologic } \\
\text { unit }\end{array}$ & $\begin{array}{l}\text { Depth } \\
\text { of } \\
\text { well } \\
\text { (ft) }\end{array}$ & $\begin{array}{c}\text { Diam- } \\
\text { oter } \\
\text { of } \\
\text { caolng } \\
\text { (In.) }\end{array}$ & $\begin{array}{c}\text { Type } \\
\text { of } \\
\text { fintah }\end{array}$ & $\begin{array}{l}\text { Top of } \\
\text { open } \\
\text { inter- } \\
\text { vel } \\
\text { (fi) }\end{array}$ & $\begin{array}{l}\text { Primary } \\
\text { use of } \\
\text { water }\end{array}$ & $\begin{array}{l}\text { Date weter } \\
\text { lovel } \\
\text { measured }\end{array}$ \\
\hline \multicolumn{10}{|c|}{ Boulder Valley-Continued } \\
\hline 05N03W22CAAA01 & 4,840 & $12-20-79$ & Pzls & 390 & 6.0 & $\mathbf{P}$ & 330 & $\mathbf{H}$ & $07-31-91$ \\
\hline 05N03W22DBBB01 & 4,848 & -- & Ts & 50 & 48.0 & - & -- & I & $07-31-91$ \\
\hline 05N03W34BACA01 & 4,682 & $03-17-76$ & Ts & 77 & 6.0 & 0 & - & $\mathbf{S}$ & $07-31-91$ \\
\hline 05N03W34BBCB01 & 4,660 & $11-30-87$ & Ts & 240 & 6.0 & $\mathbf{P}$ & 195 & H & $07-31-91$ \\
\hline 05N03W35CDCC01 & 4,635 & $04-10-78$ & Ts & 44 & 6.0 & $\mathbf{P}$ & 30 & $\mathbf{U}$ & $07-31-91$ \\
\hline 04N03W01CCAD01 & 4,590 & -- & Ts & 97 & 16.0 & - & - & I & 08-01-91 \\
\hline 04N03W01CCAD02 & 4,595 & $06-12-70$ & Ts & 98 & 6.0 & $\mathbf{P}$ & 44 & $\mathbf{S}$ & $08-01-91$ \\
\hline O4N03W 14BC $\quad 01$ & 4,695 & $09-26-88$ & Ts & 201 & 6.0 & $\mathbf{P}$ & 160 & $\mathbf{S}$ & 08-01-91 \\
\hline 04N03W25ABAB0I & 4,585 & -- & Ts & 155 & -- & - & -- & $\mathbf{H}$ & 08-28-92 \\
\hline 04N02W30CABA01 & 4,510 & 06- -91 & Unk & 181 & 6.0 & 0 & -- & U & $07-31-91$ \\
\hline 04N02W30CBCB01 & 4,560 & $02-15-91$ & Ts & 400 & 6.0 & $\mathbf{P}$ & 80 & $\mathbf{U}$ & $07-31-91$ \\
\hline 04N02W30DADD01 & 4,490 & $03-04-81$ & Pzls & 170 & 12.0 & $\mathbf{P}$ & 65 & I & -- \\
\hline 03N02W06ABA 01 & 4,550 & $10-03-73$ & Ts & 83 & 6.0 & o & -. & $\mathbf{S}$ & $07-31-91$ \\
\hline 03N02W08DCDC01 & 4,442 & 04-04-85 & Qal & 45 & 6.0 & $\mathbf{P}$ & 38 & H & 08-01-91 \\
\hline 03N02W29CDBC01 & 4,460 & $09-15-81$ & Ts & 80 & 6.0 & o & -- & $\mathbf{U}$ & 08-01-91 \\
\hline 02N03W12CACD01 & 4,400 & 08-23-89 & Ts & 117 & 6.0 & $\mathbf{P}$ & 92 & $\mathbf{H}$ & 08-01-91 \\
\hline 02N03 W12CADC01 & 4,400 & -. & Ts & 116 & 6.0 & - & - & $\mathrm{U}$ & 08-01-91 \\
\hline 02N03W15CBC 01 & 4,750 & 08-06-85 & Ts & 187 & 12.0 & $\mathbf{s}$ & 35 & I & $08-01-91$ \\
\hline 02N03W22DCDD01 & 4,520 & $08-02-72$ & Ts & 74 & 6.0 & $\mathbf{P}$ & 69 & $\mathbf{H}$ & 08-01-91 \\
\hline 02N03W23DDBD01 & 4,410 & $11-07-77$ & Yms & 215 & 6.0 & $\mathbf{P}$ & 195 & $\mathbf{H}$ & 08-01-91 \\
\hline \multicolumn{10}{|c|}{ Camas Prairie Basin } \\
\hline 20N25W03CBBA01 & 3,595 & -- & Qal & 45 & - & - & -- & $\mathbf{H}$ & 09-05-91 \\
\hline 20N24W03CCCD01 & 2,930 & -- & Qal & 68 & -- & - & -- & $\mathbf{U}$ & 08-01-91 \\
\hline 20N24W23CBAA01 & 2,797 & 09-08-84 & Qal & 99 & 6.0 & $\mathbf{P}$ & 5 & $\mathrm{U}$ & 08-02-91 \\
\hline 20N24W23CBAA02 & 2,798 & 09-09-84 & Qal & 98 & 6.0 & $\mathbf{P}$ & 11 & $\mathbf{U}$ & 08-02-91 \\
\hline 20N24W29DADD01 & 2,880 & $09-14-83$ & Qal & 54 & 2.0 & $\mathbf{P}$ & 20 & $\mathbf{U}$ & $08-01-91$ \\
\hline 20N24W34AAAB01 & 2,800 & $-\quad-73$ & Qal & 11 & - & - & -- & $\mathbf{I}$ & 08-01-91 \\
\hline 19N24W02DCDD01 & 2,800 & $09-13-83$ & Qal & 41 & 4.0 & $\mathbf{P}$ & 33 & $\mathbf{U}$ & 08-01-91 \\
\hline 19N24W02DDCD01 & 2,790 & $09-21-83$ & Qal & 24 & 2.0 & $\mathbf{P}$ & 17 & $\mathbf{U}$ & $08-01-91$ \\
\hline \multicolumn{10}{|c|}{ Centennial Valley } \\
\hline 13S06W25DBCB01 & 6,790 & $10-23-63$ & Qal & 105 & 6.0 & 0 & - & $\mathbf{H}$ & $10-23-63$ \\
\hline 13S04W17BCDA01 & 6,860 & $06-25-87$ & Ts & 115 & 6.0 & $\mathrm{X}$ & 46 & $\mathbf{H}$ & 09-19-91 \\
\hline 13S04W28CDDB01 & 6,705 & -- & QTsu & 30 & 6.0 & - & -- & $\mathbf{H}$ & $09-19-91$ \\
\hline 13S03W34BBCC01 & 6,625 & $08-26-91$ & Qal & 60 & 6.0 & $\mathbf{s}$ & 40 & $\mathbf{H}$ & 09-19-91 \\
\hline 13S02W12BCCC01 & 6,932 & -- & Qal & -- & -- & - & -- & $\mathbf{s}$ & -. \\
\hline
\end{tabular}


Table 1. Records of selected wells by basin, Northern Rocky Mountains intermontane basins, Montana (Continued)

\begin{tabular}{|c|c|c|c|c|c|c|c|c|c|}
\hline $\begin{array}{c}\text { Water lovel } \\
\text { (ft) }\end{array}$ & $\begin{array}{l}\text { Dio- } \\
\text { charge } \\
\text { (gal/ } \\
\text { min) }\end{array}$ & $\begin{array}{c}\text { Specific } \\
\text { capacity } \\
\text { [(gal/ } \\
\text { min)/ft] }\end{array}$ & $\begin{array}{l}\text { Source } \\
\text { of dis- } \\
\text { charge } \\
\text { date }\end{array}$ & $\begin{array}{l}\text { Typo } \\
\text { of log } \\
\text { avail- } \\
\text { abio }\end{array}$ & $\begin{array}{c}\text { Date } \\
\text { water. } \\
\text { quality } \\
\text { paramoter } \\
\text { measured }\end{array}$ & $\begin{array}{c}\text { Specific } \\
\text { conduc- } \\
\text { tence } \\
(\mu S / \mathrm{cm})\end{array}$ & $\begin{array}{l}\text { pH } \\
\text { (stan- } \\
\text { dard } \\
\text { unito) }\end{array}$ & $\begin{array}{l}\text { Tom- } \\
\text { per- } \\
\text { sture } \\
\left({ }^{\circ} \mathrm{C}\right)\end{array}$ & Well number \\
\hline
\end{tabular}

\begin{tabular}{|c|c|c|c|c|c|c|c|c|c|}
\hline \\
\hline 79.94 & 12 & .1 & $\mathrm{D}$ & D & $07-31-91$ & 259 & 7.2 & -- & 05N03W22CAAA01 \\
\hline 19.00 & - & -- & - & - & $07-31-91$ & 1,100 & 7.7 & 9.0 & 05N03W22DBBB01 \\
\hline 7.39 & 10 & .2 & $\mathrm{D}$ & D & $07-31-91$ & 596 & 7.6 & 19.0 & 05N03W34BACA01 \\
\hline 10.13 & 8 & .1 & $\mathrm{D}$ & D & $07-31-91$ & 289 & 8.2 & 9.0 & 05N03W34BBCB0I \\
\hline 27.48 & 10 & 1.1 & D & D & -- & -- & -- & -- & 05N03W35CDCC01 \\
\hline$* 40$ & 2,200 & -. & 0 & - & $08-01-91$ & 436 & 7.9 & 13.0 & 04N03W01CCAD01 \\
\hline 33.67 & 1,000 & 15 & D & D & -- & -- & -- & -- & 04N03W01CCAD02 \\
\hline 82.61 & 20 & -. & D & D & 08-01-91 & 282 & 8.1 & 12.0 & 04N03W 14BC $\quad 01$ \\
\hline 58.40 & -- & -- & - & - & 08-28-92 & 288 & 8.3 & 11.5 & 04N03W25ABAB0I \\
\hline 21.82 & -- & -- & - & - & -- & - & -- & -- & 04N02W30CABA01 \\
\hline 32.94 & 500 & 1.5 & D & D & -- & - & -- & -- & 04N02W30CBCB01 \\
\hline-- & 900 & 45 & D & D & $07-31-91$ & 352 & 7.6 & 13.0 & 04N02W30DADD01 \\
\hline 14.84 & 30 & .5 & D & D & $07-31-91$ & 336 & 7.5 & 10.0 & 03N02W06ABA 01 \\
\hline 11.50 & 15 & - & D & D & 08-01-91 & 490 & 8.0 & 12.0 & 03N02W08DCDC01 \\
\hline 47.09 & 75 & 7.5 & D & D & - & - & -- & $\cdots$ & 03N02W29CDBC01 \\
\hline 65.60 & 5 & - & D & $\mathrm{D}$ & $08-01-91$ & 894 & 7.4 & 12.0 & 02N03W I2CACDOI \\
\hline 48.49 & -- & - & - & - & - & -- & - & -- & 02N03W12CADC01 \\
\hline 14.60 & 800 & 57 & D & D & $08-01-91$ & 415 & 8.1 & 13.0 & 02N03W15CBC 01 \\
\hline 6.72 & 15 & .4 & D & D & 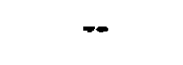 & - & -- & -- & 02N03W22DCDD01 \\
\hline 85.50 & 18 & -. & D & D & -- & -- & -- & -- & 02N03W23DDBD01 \\
\hline
\end{tabular}

\section{Camas Praire Basin}

$\begin{array}{rlllllllll}6.82 & 6 & 2.9 & \mathrm{~S} & - & 10-13-83 & 158 & 6.7 & 9.0 & \text { 20N25W03CBBA01 } \\ 49.07 & -- & -- & - & - & - & - & - & - & \text { 20N24W03CCCD01 } \\ .42 & 74 & 12 & \mathrm{~S} & \mathrm{D} & 10-11-84 & 312 & 7.6 & 10.0 & \text { 20N24W23CBAA01 } \\ .35 & 80 & 45 & \mathrm{~S} & \mathrm{D} & 10-12-84 & 325 & 7.8 & 10.0 & \text { 20N24W23CBAA02 } \\ 3.90 & -- & -- & - & \mathrm{D} & - & - & -- & - & \text { 20N24W29DADD01 } \\ & - & & & & & & & & \\ 2.89 & -- & -- & - & - & 07-09-75 & 375 & -- & 11.0 & \text { 20N24W34AAAB01 } \\ 11.01 & -- & -- & - & \mathrm{D} & - & -- & -- & -- & \text { 19N24W02DCDD01 } \\ 5.00 & -- & -- & - & \mathrm{D} & -- & -- & -- & -- & \text { 19N24W02DDCD01 }\end{array}$

\section{Centennial Valley}

$\begin{array}{cccccccccc}* 25 & 5 & .1 & \mathrm{D} & \mathrm{D} & - & - & -- & -- & \text { 13SO6W25DBCB01 } \\ 7.72 & 35 & -- & \mathrm{D} & \mathrm{D} & 09-19-91 & 572 & -- & 6.5 & \text { 13S04W 17BCDA01 } \\ 15.32 & -- & -- & - & - & 09-19-91 & 461 & -- & 7.0 & \text { 13S04W28CDDB01 } \\ 13.30 & 15 & -4 & \mathrm{D} & \mathrm{D} & -- & -- & -- & -- & \text { 13S03W34BBCC01 } \\ -- & - & -- & - & - & -- & - & -- & -- & \text { 13S02W12BCCC01 }\end{array}$


Table 1. Records of selected wells by basin, Northern Rocky Mountains intermontane basins, Montana (Continued)

\begin{tabular}{|c|c|c|c|c|c|c|c|c|c|}
\hline Well number & $\begin{array}{c}\text { Altitude } \\
\text { of land } \\
\text { surface } \\
\text { (ft) }\end{array}$ & $\begin{array}{c}\text { Date well } \\
\text { constructed }\end{array}$ & $\begin{array}{c}\text { Geologic } \\
\text { unit }\end{array}$ & $\begin{array}{c}\text { Depth } \\
\text { of } \\
\text { well } \\
\text { (ft) }\end{array}$ & $\begin{array}{c}\text { Diam- } \\
\text { oter } \\
\text { of } \\
\text { casing } \\
\text { (In.) }\end{array}$ & $\begin{array}{l}\text { Type } \\
\text { of } \\
\text { finlsh }\end{array}$ & $\begin{array}{l}\text { Top of } \\
\text { Open } \\
\text { Inter- } \\
\text { val } \\
\text { (ft) }\end{array}$ & $\begin{array}{l}\text { Primary } \\
\text { use of } \\
\text { water }\end{array}$ & $\begin{array}{c}\text { Date water } \\
\text { level } \\
\text { measured }\end{array}$ \\
\hline \multicolumn{10}{|c|}{ Centennial Valley-Continued } \\
\hline 13S02W 12CBBA01 & 6,950 & $08-31-88$ & QTKe & 186 & 6.0 & $\mathbf{P}$ & 168 & $\mathbf{H}$ & $09-17-91$ \\
\hline 13S02W 14BDCA01 & 6,710 & $07-29-63$ & Qal & 92 & 6.0 & o & - & $\mathbf{S}$ & -- \\
\hline 13S02W17CCBD01 & 6,745 & $01-01-60$ & Qal & 38 & 8.0 & o & -- & $\mathbf{H}$ & $09-17-91$ \\
\hline 13S02W19CDDC01 & 6,654 & $09-01-91$ & Qal & 60 & 6.6 & $\mathbf{P}$ & 40 & $\mathbf{H}$ & $09-20-91$ \\
\hline 13S02W20AACA01 & 6,709 & -- & QTsu & -- & -- & - & -- & $\mathbf{S}$ & $09-19-91$ \\
\hline 13S02W20ADCA01 & 6,675 & -- & Qal & -- & - & - & -- & $\mathbf{S}$ & $09-19-91$ \\
\hline 13S02W24DCCCO1 & 6,660 & $01-01-37$ & Qal & 207 & 6.0 & 0 & -- & $\mathbf{s}$ & $09-18-91$ \\
\hline 13S01W27BBAC01 & 6,700 & $06-27-63$ & Qal & 86 & 6.0 & $\mathrm{O}$ & -- & $\mathbf{S}$ & $09-18-91$ \\
\hline 13S01 W28BCBB01 & 6,665 & -- & Qal & -- & -- & - & -- & $\mathbf{U}$ & $09-18-91$ \\
\hline 14S04W 19DAAD01 & 6,655 & $01-01-46$ & Qal & 40 & 6.0 & o & -- & $\mathbf{H}$ & $09-18-91$ \\
\hline 14S03W13DBCB01 & 6,650 & $10-02-61$ & Qal & 59 & 6.0 & $\mathrm{o}$ & -- & $\mathbf{U}$ & 09-18-91 \\
\hline 14S03W21DDCC01 & 6,690 & $09-15-70$ & Qal & 53 & 6.0 & $\mathrm{o}$ & -. & $\mathbf{H}$ & $09-18-91$ \\
\hline 14S03W 22CABD01 & 6,670 & $12-24-75$ & Qal & 148 & 6.0 & $\mathbf{P}$ & 132 & $\mathbf{S}$ & $09-18-91$ \\
\hline 14S02W23BABA01 & 6,700 & -- & QTsu & - & 6.0 & - & -- & $\mathbf{H}$ & 09-20-91 \\
\hline 14S02W23BDDA01 & 6,710 & -- & QTsu & 105 & -- & - & - & $\mathbf{H}$ & $09-18-91$ \\
\hline 14S01E22ACBB01 & 6,780 & 09-06-88 & Qal & 99 & 6.0 & $\mathbf{o}$ & -- & $\mathbf{H}$ & 09-19-91 \\
\hline 14S01E22DBAA01 & 6,845 & $06-24-85$ & Qal & 138 & 8.0 & $\mathbf{O}$ & - & $\mathbf{H}$ & 09-20-91 \\
\hline 14S01E24CDAA01 & 6,900 & 08-02-85 & Qal & 158 & 8.0 & $\mathrm{O}$ & -- & $\mathbf{H}$ & $09-20-91$ \\
\hline \multicolumn{10}{|c|}{ Gallatin Valley } \\
\hline 03N04E24BDAA01 & 4,820 & $10-10-86$ & Qal & 65 & 6.0 & o & -- & $\mathbf{H}$ & $06-21-91$ \\
\hline 02N02E15BCDB01 & 4,090 & $07-13-83$ & QTsu & 103 & 6.0 & O & -- & $\mathbf{H}$ & $06-25-91$ \\
\hline 02N02E27CDDC01 & 4,110 & $05-24-78$ & Ts & 200 & 16.0 & $\mathbf{P}$ & 45 & I & $05-24-78$ \\
\hline 02N02E35ADDA01 & 4,143 & $10-07-86$ & Pzls & 165 & 6.0 & $\mathbf{P}$ & 125 & H & $06-24-91$ \\
\hline 02N02E36BCAA01 & 4,102 & $01-06-88$ & Ts & 142 & 6.0 & $\mathbf{P}$ & 58 & HI & $06-25-91$ \\
\hline 02N04E23DBDD01 & 4,442 & $08-17-79$ & Ts & 62 & 6.0 & $\mathbf{P}$ & 39 & $\mathbf{H}$ & $06-24-91$ \\
\hline 02N05E04DADD01 & 5,310 & $10-20-73$ & Ts & 83 & 6.0 & $\mathbf{P}$ & 62 & $\mathbf{H}$ & $06-21-91$ \\
\hline 02N05E06ADDA01 & 4,892 & 05-28-82 & Ts & 53 & 6.0 & o & - & $\mathbf{H}$ & $06-21-91$ \\
\hline 02N05E10BDBD01 & 5,530 & $07-29-81$ & Ts & 294 & 6.0 & $\mathrm{o}$ & -- & $\mathbf{H}$ & $06-21-91$ \\
\hline 02N05E34DDBB01 & 4,740 & $08-06-87$ & Ts & 300 & 6.0 & $\mathbf{P}$ & 152 & I & $06-20-91$ \\
\hline 01N02E14CADC01 & 4,620 & -- & Ts & -- & 6.0 & - & -- & $\mathbf{U}$ & $06-24-91$ \\
\hline 01N03E10ABBA01 & 4,238 & $08-28-65$ & Ts & 327 & 16.0 & $\mathbf{F}$ & 99 & $\mathbf{P}$ & 05-02-91 \\
\hline OIN03E1OADCD01 & 4,250 & $04-28-58$ & Ts & 224 & 13.0 & $\mathbf{x}$ & 75 & $\mathbf{H}$ & -- \\
\hline 01 N03E16CABB0I & 4,375 & $06-21-78$ & Ts & 400 & 16.0 & $\mathbf{P}$ & 39 & $\mathbf{U}$ & $05-14-91$ \\
\hline 01N03E19ADBB01 & 4,525 & $10-08-90$ & Ts & 526 & 16.0 & $\mathbf{F}$ & 346 & I & $10-08-80$ \\
\hline
\end{tabular}


Table 1. Records of selected wells by basin, Northern Rocky Mountains intermontane basins, Montana (Continued)

\begin{tabular}{|c|c|c|c|c|c|c|c|c|c|}
\hline $\begin{array}{c}\text { Water level } \\
\text { (ft) }\end{array}$ & $\begin{array}{c}\text { Dis- } \\
\text { charge } \\
\text { (gall } \\
\text { min) }\end{array}$ & $\begin{array}{c}\text { Specific } \\
\text { capacity } \\
\text { [(gal/ } \\
\text { min)/ft] }\end{array}$ & $\begin{array}{l}\text { Source } \\
\text { of dis- } \\
\text { charge } \\
\text { data }\end{array}$ & $\begin{array}{l}\text { Type } \\
\text { of log } \\
\text { avall- } \\
\text { able }\end{array}$ & $\begin{array}{c}\text { Date } \\
\text { water- } \\
\text { quality } \\
\text { parameter } \\
\text { measured }\end{array}$ & $\begin{array}{c}\text { Specific } \\
\text { conduc- } \\
\text { tance } \\
(\mu \mathrm{S} / \mathrm{cm})\end{array}$ & $\begin{array}{l}\text { pH } \\
\text { (stan- } \\
\text { dard } \\
\text { units) }\end{array}$ & $\begin{array}{l}\text { Tom- } \\
\text { per- } \\
\text { ature } \\
\left({ }^{\circ} \mathrm{C}\right)\end{array}$ & Well number \\
\hline
\end{tabular}

\begin{tabular}{|c|c|c|c|c|c|c|c|c|c|}
\hline \\
\hline 155.40 & 5 & -- & D & D & 09-17-91 & 372 & -- & 11.5 & 13S02W12CBBA01 \\
\hline -- & 20 & 2.0 & D & D & -- & -- & -- & -- & 13S02W 14BDCA01 \\
\hline 15.90 & 35 & -- & D & D & $09-17-91$ & 797 & -. & 11.0 & 13S02W17CCBD01 \\
\hline 6.94 & 25 & 2.1 & $\mathrm{D}$ & D & - & - & -- & -- & 13S02W19CDDC01 \\
\hline 66.32 & - & -- & - & - & 09-19-91 & 547 & -. & 7.5 & 13S02W20AACA01 \\
\hline 30.69 & - & -- & - & - & $09-19-91$ & 567 & -- & 6.5 & 13S02W20ADCA01 \\
\hline 16.63 & 12 & -- & $\mathrm{D}$ & - & $09-19-91$ & 297 & - & 8.0 & 13S02W24DCCCO1 \\
\hline 63.90 & 20 & 2.0 & $\mathrm{D}$ & D & $09-19-91$ & 258 & 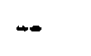 & - & 13S01W27BBAC01 \\
\hline 12.85 & -- & -- & - & - & 09-18-91 & 163 & - & 9.5 & 13S0I W28BCBB0I \\
\hline 17.94 & 460 & -- & $\mathrm{D}$ & $\mathbf{D}$ & $09-18-91$ & 427 & - & 6.0 & 14S04W 19DAAD01 \\
\hline 9.25 & 12 & -- & D & D & -- & -- & -- & - & 14S03W13DBCB01 \\
\hline 35.47 & 20 & 1.0 & D & D & $09-18-91$ & 576 & -- & 5.5 & 14S03W21DDCC01 \\
\hline 23.68 & 15 & 7.5 & D & D & -- & - & -- & -- & 14S03W22CABD01 \\
\hline 35.39 & -- & -- & - & - & $09-20-91$ & 478 & -- & 5.5 & 14S02W23BABA01 \\
\hline 40.72 & -- & -- & - & - & $09-18-91$ & 135 & -- & 5.5 & 14S02W23BDDA01 \\
\hline 24.69 & -- & -- & - & $\mathrm{D}$ & $09-19-91$ & 208 & -- & 5.0 & 14S01E22ACBB01 \\
\hline 64.35 & - & -. & - & D & $\cdots$ & -- & -- & -- & 14S01E22DBAA01 \\
\hline 94.89 & -- & -- & - & $\mathrm{D}$ & $09-20-91$ & 302 & - & 5.0 & 14S01E24CDAA01 \\
\hline \multicolumn{10}{|c|}{ Gallatin Valley } \\
\hline 24.58 & 15 & -- & D & D & $06-21-91$ & 610 & 7.6 & 9.0 & 03N04E24BDAA01 \\
\hline 39.32 & 20 & .5 & D & D & $06-24-91$ & 554 & 7.6 & 12.0 & 02N02E15BCDB01 \\
\hline 34.00 & - & -- & - & D & -- & -- & -- & -- & 02N02E27CDDC01 \\
\hline 72.43 & 5 & .1 & D & D & $06-24-91$ & 165 & 7.5 & 9.5 & 02N02E35ADDA01 \\
\hline 22.27 & 15 & .9 & $\mathrm{D}$ & D & $06-25-91$ & 616 & 7.4 & 12.0 & 02N02E36BCAA01 \\
\hline 18.57 & 7 & .5 & $\mathbf{s}$ & D & $06-21-91$ & 601 & 7.3 & 10.0 & 02N04E23DBDD01 \\
\hline 59.15 & 30 & 7.5 & D & D & $06-21-91$ & 329 & 7.5 & 8.5 & 02N05E04DADD01 \\
\hline 37.85 & 30 & -- & D & D & $06-21-91$ & 412 & 7.3 & 11.5 & 02N05E06ADDA01 \\
\hline 253.95 & 25 & 3.1 & D & D & $06-21-91$ & 305 & 7.6 & 8.0 & 02N05E10BDBD01 \\
\hline 13.01 & 25 & .8 & $\mathrm{D}$ & D & $06-20-91$ & 283 & 7.6 & 11.0 & 02N05E34DDBB01 \\
\hline 458.60 & - & -- & - & - & -. & -. & -- & -. & 01N02E14CADC01 \\
\hline 23.25 & 500 & 5.7 & D & $\mathrm{D}$ & -- & - & -- & - & 01N03E10ABBA01 \\
\hline- & 520 & 7.4 & $\mathbf{R}$ & $\mathrm{D}$ & -- & -- & -- & -- & 01N03E10ADCD01 \\
\hline 84.56 & -- & -- & - & D & $\cdots$ & -- & -- & -- & 01N03E16CABB01 \\
\hline *310 & 1,600 & 13 & D & D & -- & - & -- & -- & 01N03E19ADBB01 \\
\hline
\end{tabular}


Table 1. Records of selected wells by basin, Northern Rocky Mountains intermontane basins, Montana (Continued)

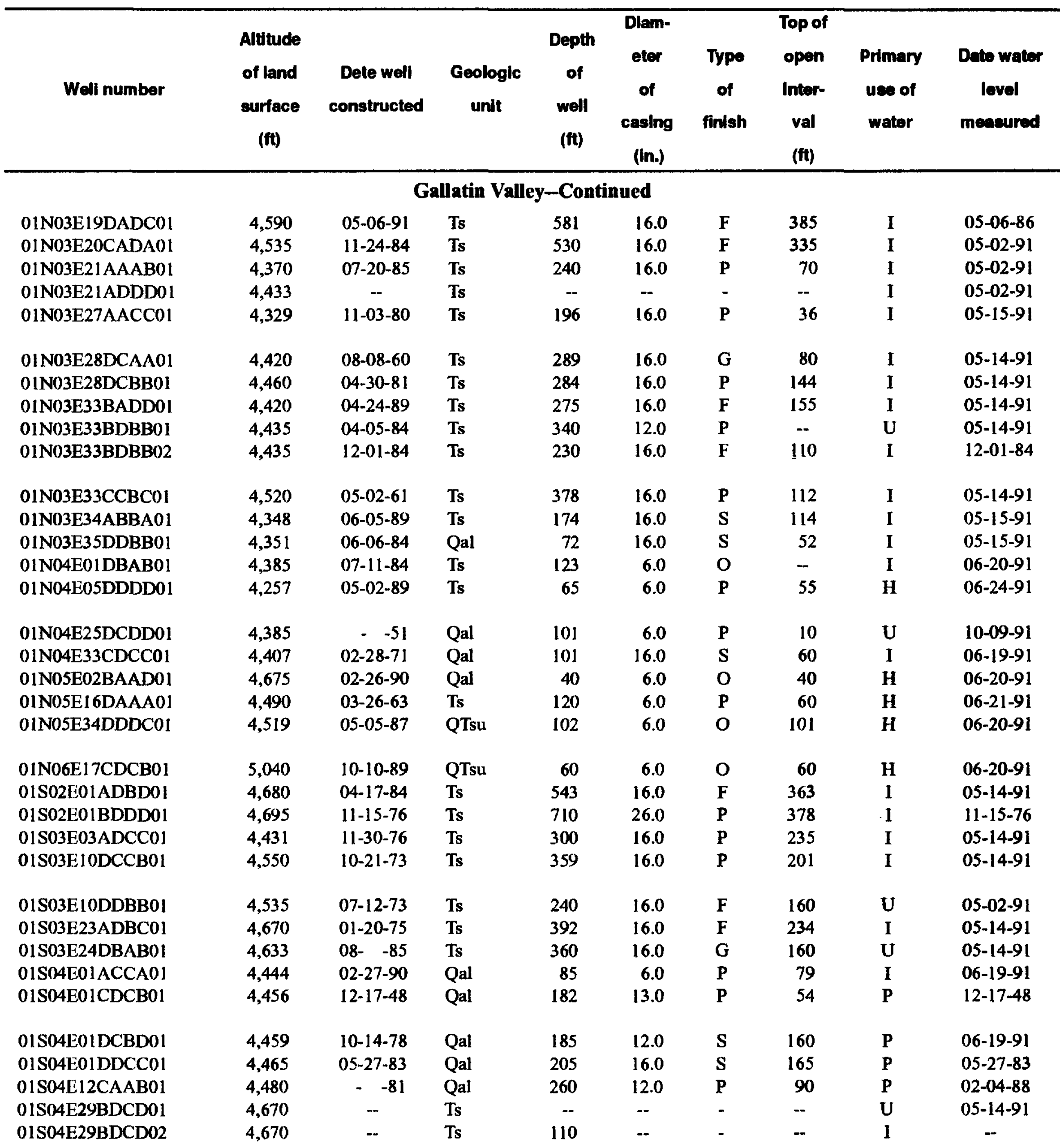


Table 1. Records of selected wells by basin, Northern Rocky Mountains intermontane basins, Montana (Continued)

\begin{tabular}{|c|c|c|c|c|c|c|c|c|c|}
\hline $\begin{array}{c}\text { Water levei } \\
\text { (fi) }\end{array}$ & $\begin{array}{c}\text { Dis- } \\
\text { charge } \\
\text { (gal/ } \\
\text { min) }\end{array}$ & $\begin{array}{c}\text { Specific } \\
\text { capacity } \\
\text { [(gaV } \\
\text { m(n)/ft] }\end{array}$ & $\begin{array}{l}\text { Source } \\
\text { of dis- } \\
\text { charge } \\
\text { data }\end{array}$ & $\begin{array}{l}\text { Type } \\
\text { of log } \\
\text { avall- } \\
\text { abie }\end{array}$ & $\begin{array}{c}\text { Date } \\
\text { water- } \\
\text { quality } \\
\text { parameter } \\
\text { measured }\end{array}$ & $\begin{array}{c}\text { Specific } \\
\text { conduc- } \\
\text { tance } \\
\text { ( } \mu \mathrm{S} / \mathrm{cm})\end{array}$ & $\begin{array}{l}\text { pH } \\
\text { (stan- } \\
\text { dard } \\
\text { unlts) }\end{array}$ & $\begin{array}{l}\text { Tom- } \\
\text { per- } \\
\text { ature } \\
\left({ }^{\circ} \mathrm{C}\right)\end{array}$ & Well number \\
\hline
\end{tabular}

\begin{tabular}{|c|c|c|c|c|c|c|c|c|c|}
\hline \multicolumn{10}{|c|}{ Gallatin Valley-Continued } \\
\hline$* 350$ & 920 & 8.2 & D & D & -- & -- & -- & -- & 01N03E19DADC01 \\
\hline 299.48 & 1,500 & -- & D & D & - & - & -. & -- & 0IN03E20CADA0I \\
\hline 66.87 & 1,300 & 11 & D & D & -- & -- & -- & - & 01N03E21AAAB01 \\
\hline 121.20 & -- & -- & - & - & $\cdots$ & -. & - & -. & 01N03E21ADDD01 \\
\hline 14.36 & 2,000 & 35 & $\mathbf{L}$ & D & -- & - & -- & -- & 01N03E27AACC01 \\
\hline 77.30 & 2,000 & 59 & $\mathrm{D}$ & $\mathbf{D}$ & -- & -- & -- & -- & 01N03E28DCAA01 \\
\hline 115.39 & 1,400 & 23 & D & D & -- & - & -- & - & 01N03E28DCBB01 \\
\hline 78.92 & 1,700 & 23 & D & D & $-\infty$ & - & - & -- & 01N03E33BADD01 \\
\hline 17.42 & -- & -- & - & D & -- & -- & -- & -- & 01N03E33BDBB01 \\
\hline$* 70$ & 1,700 & 21 & $\mathrm{D}$ & $\mathbf{D}$ & -- & -- & -- & - & 01N03E33BDBB02 \\
\hline 145.08 & 1,000 & 9.3 & $\mathbf{L}$ & $\mathbf{D}$ & -- & - & -- & -- & 01N03E33CCBC01 \\
\hline 18.47 & 1,900 & 23 & $\mathrm{D}$ & D & -- & -- & -- & -- & 01N03E34ABBA01 \\
\hline 11.45 & 900 & 16 & $\mathrm{D}$ & D & -- & -- & .. & -. & 01N03E35DDBB01 \\
\hline 75.73 & 7 & .4 & $\mathbf{S}$ & D & $06-19-91$ & 309 & 7.6 & 12.0 & 01N04E0 1DBAB01 \\
\hline 8.21 & 30 & .6 & D & $\mathbf{D}$ & $06-24-91$ & 462 & 7.5 & 9.5 & 01N04E05DDDD01 \\
\hline 12.98 & 140 & 20 & $\mathbf{S}$ & - & $12-07-51$ & - & - & 11.0 & 01N04E25DCDD01 \\
\hline 21.85 & 1,500 & 45 & D & D & -- & -- & .- & -. & 01N04E33CDCC01 \\
\hline 5.97 & 25 & 1.3 & $\mathrm{D}$ & D & $06-20-91$ & 240 & 7.0 & 10.0 & 01N05E02BAAD01 \\
\hline 43.33 & 13 & 1.3 & $\mathbf{S}$ & D & $06-21-91$ & 461 & 7.1 & 8.0 & 01N05E16DAAA01 \\
\hline$* 64$ & 25 & 1.2 & D & D & $06-20-91$ & 315 & 7.7 & 9.0 & 01N05E34DDDC01 \\
\hline 10.77 & 30 & .8 & D & D & $06-20-91$ & 328 & 7.4 & 7.5 & 01N06E1 7CDCB01 \\
\hline 276.23 & 990 & 5.4 & D & D & -. & -- & -. & -- & 01S02E01ADBD01 \\
\hline *303 & 1,900 & 9.2 & D & D & $08-14-91$ & 388 & 7.8 & -- & 01S02E01BDDD01 \\
\hline 79.35 & 2,800 & 21 & D & D & -- & -- & .. & -- & 01S03E03ADCC01 \\
\hline 140.47 & 900 & 12 & D & D & -- & -- & -- & -- & 01S03E10DCCB01 \\
\hline 148.25 & 630 & 18 & D & D & -- & -- & -- & - & 01S03E10DDBB01 \\
\hline 177.64 & 600 & 7.0 & D & D & -- & -- & -- & -- & 01S03E23ADBC01 \\
\hline 142.79 & 1,200 & 14 & $\mathbf{D}$ & D & -- & - & -- & -- & 01S03E24DBAB01 \\
\hline 47.64 & 30 & 1.6 & $\mathbf{D}$ & D & $06-19-91$ & 450 & 7.6 & 10.5 & 01S04E01ACCA01 \\
\hline 47.40 & 750 & -- & 0 & - & -- & -- & - & - & 01S04E01CDCB01 \\
\hline 54.73 & 600 & 5.1 & D & D & - & -- & - & -. & 01S04E01DCBD01 \\
\hline 57.00 & -- & -- & - & D & -- & -. & -. & -- & 01S04E01DDCC01 \\
\hline 53.40 & 730 & 6.7 & D & D & 06-19-91 & 432 & 7.6 & 10.5 & 01S04E12CAAB01 \\
\hline 69.48 & -- & -- & - & - & -. & -- & -- & -- & 01S04E29BDCD01 \\
\hline$\ldots$ & 1,600 & .- & $\Lambda$ & - & -- & -- & -. & -- & 01S04E29BDCD02 \\
\hline
\end{tabular}


Table 1. Records of selected wells by basin, Northern Rocky Mountains intermontane basins, Montana (Continued)

\begin{tabular}{|c|c|c|c|c|c|c|c|c|c|}
\hline Well number & $\begin{array}{l}\text { Altitude } \\
\text { of land } \\
\text { surface } \\
\text { (fi) }\end{array}$ & $\begin{array}{l}\text { Date well } \\
\text { constructed }\end{array}$ & $\begin{array}{c}\text { Geologic } \\
\text { unit }\end{array}$ & $\begin{array}{c}\text { Depth } \\
\text { of } \\
\text { well } \\
\text { (ft) }\end{array}$ & $\begin{array}{c}\text { Diam- } \\
\text { oter } \\
\text { of } \\
\text { casing } \\
\text { (In.) }\end{array}$ & $\begin{array}{c}\text { Type } \\
\text { of } \\
\text { finish }\end{array}$ & $\begin{array}{l}\text { Top of } \\
\text { open } \\
\text { Inter- } \\
\text { val } \\
\text { (ft) }\end{array}$ & $\begin{array}{c}\text { Primary } \\
\text { use of } \\
\text { water }\end{array}$ & $\begin{array}{l}\text { Date water } \\
\text { level } \\
\text { measured }\end{array}$ \\
\hline \multicolumn{10}{|c|}{ Gallatin Valley-Continued } \\
\hline 01S05E16DBCC01 & 4,525 & $09-19-85$ & Qal & 59 & 6.0 & $\mathbf{P}$ & 40 & H & $06-18-91$ \\
\hline OIS06E1 8BBBB01 & 4,850 & $03-24-78$ & QTsu & 285 & 6.0 & o & .- & $\mathbf{H}$ & $06-20-91$ \\
\hline 01S06E20BDAA01 & 5,150 & --71 & QTsu & 110 & 6.0 & - & -- & $\mathbf{H}$ & $06-20-91$ \\
\hline 01S06E20BDAB01 & 5,135 & $08-29-90$ & QTsu & 166 & 6.6 & $\mathbf{P}$ & 145 & $\mathbf{H}$ & $06-20-91$ \\
\hline 0IS06E20CBDD0 I & 5,090 & $09-24-84$ & QTsu & 230 & 6.0 & 0 & 110 & $\mathbf{H}$ & $06-20-91$ \\
\hline 02S03E04BAAD01 & 4,605 & $08-09-78$ & Ts & 120 & 16.0 & $\mathbf{F}$ & 22 & I & $05-15-91$ \\
\hline 02S04E05BCBB01 & 4,780 & $11-28-78$ & Ts & 178 & 6.0 & o & -- & $\mathbf{H}$ & $06-23-91$ \\
\hline 02S04E09BCCC01 & 4,905 & $-\quad-51$ & Ts & 600 & 6.0 & - & -. & $\mathbf{H}$ & $02-08-52$ \\
\hline 02S04E13CCBC01 & 4,738 & $-\quad-47$ & Qal & 11 & 12.0 & o & -- & $\mathbf{U}$ & $06-23-91$ \\
\hline 02S04EI4DADC03 & 4,745 & --80 & Unk & 685 & 10.0 & - & -- & $\mathbf{R}$ & $06-23-91$ \\
\hline 02S04E29CDDC0I & 5,221 & -- & Ts & 56 & 6.0 & - & $\ldots$ & $\mathbf{S}$ & $06-22-91$ \\
\hline 02S05E05ABBB0I & 4,688 & $02-21-89$ & Qal & 40 & 6.0 & o & -- & $\mathbf{H}$ & $06-23-91$ \\
\hline 02S05E05ABBD01 & 4,686 & $10-31-77$ & Ts & 70 & 6.0 & o & -- & $\mathbf{H}$ & $06-23-91$ \\
\hline 02S05E20DDCC01 & 4,945 & $12-16-72$ & Qal & 30 & 6.0 & o & - & H & $06-23-91$ \\
\hline 02S06E08ADDA0I & 5,090 & $08-15-85$ & Ts & 550 & 6.0 & $\mathbf{P}$ & 530 & $\mathbf{H}$ & $06-24-91$ \\
\hline 02S06E20CABD01 & 5,100 & -- & Ts & 300 & -- & - & -- & $\mathbf{H}$ & $06-22-91$ \\
\hline 02S06Е20CACA01 & 5,132 & $02-23-87$ & Ts & 238 & 6.0 & o & -- & $\mathbf{H}$ & $06-22-91$ \\
\hline 02S06E26DDBA01 & 5,150 & $01-24-90$ & QTsu & 70 & 6.0 & 0 & -- & HI & $06-23-91$ \\
\hline 02S06E33ADAC01 & 5,420 & 08-15-85 & Ts & 176 & 6.0 & o & -- & $\mathrm{U}$ & $06-22-91$ \\
\hline 02S06E33BABDO1 & 5,440 & $11-21-86$ & QTsu & 305 & 6.0 & $\mathbf{s}$ & 265 & $\mathbf{H}$ & $06-22-91$ \\
\hline 02S06E33BDDC01 & 5,435 & $08-13-85$ & Ts & 159 & 6.0 & $\mathbf{P}$ & 132 & $\mathbf{U}$ & $06-22-91$ \\
\hline 03S04EIICBBA0I & 4,910 & $03-20-90$ & Qal & 40 & 6.0 & o & -- & $\mathbf{H}$ & $06-18-91$ \\
\hline 03S04E2 IDDBD0I & 5,031 & $03-31-71$ & QTsu & 105 & 16.0 & $\mathbf{s}$ & 70 & $\mathbf{1}$ & $03-3 \mid-71$ \\
\hline 03S04E22CBDC01 & 5,012 & $10-03-70$ & Qal & 31 & 6.0 & o & -- & $\mathbf{S}$ & $06-18-91$ \\
\hline 03S04E25DBBD0 1 & 5,285 & $07-23-85$ & Qal & 50 & 6.0 & o & - & $\mathbf{H}$ & $06-18-91$ \\
\hline 03S04E32DADA01 & 5,150 & $03-24-79$ & Ts & 71 & 6.0 & $\mathbf{P}$ & 58 & $\mathbf{H}$ & $06-18-91$ \\
\hline 03S04E36BABB0I & 5,487 & $07-26-83$ & Ts & 312 & 6.0 & $\mathbf{P}$ & 306 & $\mathbf{H}$ & $06-18-91$ \\
\hline 03S05E07BCCCO1 & 5,100 & $06-29-90$ & Qal & 36 & 6.0 & 0 & -- & $\mathbf{H}$ & $06-18-91$ \\
\hline 03S05E10СССB01 & 5,312 & -- & Qal & 70 & -- & - & -- & $\mathbf{H}$ & $06-22-91$ \\
\hline 03S05EIIDABD01 & 5,390 & $08-30-73$ & QTsu & 72 & 6.0 & o & -- & $\mathbf{U}$ & $06-22-91$ \\
\hline 03S05E15DCCA01 & 5,790 & $08-12-76$ & Ts & 220 & 6.0 & o & -- & $\mathbf{H}$ & $06-22-91$ \\
\hline 04S04E18ACAA01 & 5,210 & $08-20-76$ & Qal & 40 & 6.0 & $\mathbf{P}$ & 30 & $\mathbf{H}$ & $11-02-92$ \\
\hline \multicolumn{10}{|c|}{ Grasshopper Valley } \\
\hline 04S12W29CCCA01 & 6,925 & $07-08-78$ & TKi & 150 & 6.0 & o & - & $\mathbf{P}$ & $10-08-91$ \\
\hline 05S 12W08AADC01 & 6,595 & $02-01-86$ & QTsu & 43 & 6.0 & $\mathbf{P}$ & 14 & $\mathbf{H}$ & $10-08-91$ \\
\hline 05S 12 W08CBAA01 & 6,590 & $05-31-71$ & QTsu & 128 & 8.0 & $\mathbf{P}$ & 38 & $\mathbf{P}$ & $10-08-91$ \\
\hline 05S12W17CBBA01 & 6,660 & $10-15-89$ & Ts & 196 & 6.0 & $\mathbf{P}$ & 190 & $\mathbf{H}$ & $10-08-91$ \\
\hline 05S 12 W 29BCDC0I & 6,370 & $06-24-81$ & Qal & 74 & 6.0 & $\mathbf{P}$ & 66 & $\mathbf{H}$ & $10-08-91$ \\
\hline
\end{tabular}


Table 1. Records of selected wells by basin, Northern Rocky Mountains intermontane basins, Montana (Continued)

\begin{tabular}{|c|c|c|c|c|c|c|c|c|c|}
\hline $\begin{array}{c}\text { Water level } \\
\text { (ft) }\end{array}$ & $\begin{array}{c}\text { Dls- } \\
\text { charge } \\
\text { (gal/ } \\
\text { min) }\end{array}$ & $\begin{array}{c}\text { Specific } \\
\text { capacity } \\
\text { [(gal } \\
\text { min)/ft] }\end{array}$ & $\begin{array}{l}\text { Sourca } \\
\text { of dis- } \\
\text { charge } \\
\text { data }\end{array}$ & $\begin{array}{l}\text { Type } \\
\text { of log } \\
\text { avail- } \\
\text { able }\end{array}$ & $\begin{array}{c}\text { Data } \\
\text { water- } \\
\text { quallty } \\
\text { paramater } \\
\text { measured }\end{array}$ & $\begin{array}{c}\text { Specific } \\
\text { conduc- } \\
\text { tance } \\
(\mu S / \mathrm{cm})\end{array}$ & $\begin{array}{l}\text { pH } \\
\text { (stan- } \\
\text { dard } \\
\text { units) }\end{array}$ & $\begin{array}{l}\text { Tem- } \\
\text { per- } \\
\text { ature } \\
\left({ }^{\circ} \mathrm{C}\right)\end{array}$ & Well number \\
\hline
\end{tabular}

\begin{tabular}{|c|c|c|c|c|c|c|c|c|c|}
\hline \multicolumn{10}{|c|}{ Gallatin Valley_Continued } \\
\hline 37.24 & 45 & 3.8 & D & D & 06-18-91 & 575 & 7.3 & 9.5 & 01S05E16DBCC01 \\
\hline 213.91 & 60 & 1.2 & D & D & $06-20-91$ & 405 & 7.4 & 10.0 & 01S06E18BBBB01 \\
\hline 39.39 & 3 & -- & $\mathrm{O}$ & - & -- & -- & -- & -- & 01S06E20BDAA01 \\
\hline 38.38 & 20 & .2 & D & $\mathrm{D}$ & $06-20-91$ & 276 & 7.8 & 10.0 & 01S06E20BDAB01 \\
\hline 88.42 & 8 & $-\cdot$ & D & D & $06-20-91$ & 502 & 7.4 & 11.0 & $0 \mathrm{iS06E20CBDD01}$ \\
\hline 11.20 & -- & -- & - & - & -- & -- & .. & -- & 02S03E04BAAD01 \\
\hline 58.00 & 30 & .5 & D & D & $06-23-91$ & 711 & 7.5 & 10.0 & 02S04E05BCBB01 \\
\hline 25.57 & -- & -- & - & - & -- & -- & -- & -- & 02S04E09BCCCOI \\
\hline 7.55 & -- & -- & - & - & $\ldots$ & -. & - & -- & 02S04E13CCBC01 \\
\hline Flowing & 1,000 & -- & $\mathbf{A}$ & - & $06-23-91$ & 705 & 8.9 & 53.5 & 02S04E14DADC03 \\
\hline 39.35 & 6 & 1.9 & $\mathbf{S}$ & - & $06-23-91$ & 199 & 6.7 & 10.0 & 02S04E29CDDC01 \\
\hline 6.25 & 25 & .9 & D & D & $06-23-91$ & 420 & 7.2 & 9.5 & 02S05E05ABBB01 \\
\hline 2.79 & 45 & 1.2 & D & D & $06-23-91$ & 268 & 7.7 & 10.5 & 02S05E05ABBD01 \\
\hline 5.22 & 20 & 1.3 & D & D & $06-23-91$ & 433 & 7.3 & 8.5 & 02S05E20DDCC01 \\
\hline 223.05 & 10 & .1 & $\mathrm{D}$ & D & $06-24-91$ & 372 & 7.6 & 11.0 & 02S06E08ADDA01 \\
\hline 140.90 & 7 & .2 & $\mathbf{S}$ & - & $06-22-91$ & 345 & 7.8 & 9.5 & 02S06E20CABDO1 \\
\hline 167.03 & 15 & .2 & D & D & $06-22-91$ & 472 & 7.5 & 9.5 & 02S06E20CACA01 \\
\hline$* 23$ & 7 & .3 & $\mathbf{S}$ & D & $06-23-91$ & 647 & 7.0 & 7.0 & 02S06E26DDBA01 \\
\hline 88.37 & 20 & .2 & D & $\mathrm{D}$ & .. & -- & -- & -- & 02S06E33ADAC01 \\
\hline 126.44 & 23 & .2 & D & $\mathrm{D}$ & $06-22-91$ & 364 & 7.5 & 8.5 & 02S06E33BABD01 \\
\hline 31.71 & 15 & .2 & D & D & -- & -- & -. & -- & 02S06E33BDDC01 \\
\hline 5.48 & 80 & 11 & D & D & $06-18-91$ & 541 & 7.2 & 7.0 & 03S04E11CBBA01 \\
\hline$* 26$ & 1,600 & 38 & $\mathbf{D}$ & D & $06-18-91$ & 310 & 7.5 & 7.0 & 03S04E21DDBD01 \\
\hline 5.04 & 60 & 30 & D & D & -- & -- & -- & - & 03S04E22CBDC01 \\
\hline 15.81 & 15 & .5 & $\mathrm{D}$ & $\mathbf{D}$ & $06-18-91$ & 299 & 7.2 & 5.5 & 03S04E25DBBD01 \\
\hline 53.28 & 7 & 1.2 & $\mathbf{S}$ & D & $06-18-91$ & 576 & 7.1 & 9.0 & 03S04E32DADA01 \\
\hline 233.40 & 17 & .3 & D & D & $06-18-91$ & 340 & 7.2 & 9.5 & 03S04E36BABB01 \\
\hline 3.87 & 12 & 23 & $\mathbf{S}$ & D & $06-18-91$ & 235 & 7.5 & 7.5 & 03S05E07BCCC01 \\
\hline 41.66 & 5 & 1.1 & $\mathbf{S}$ & - & $06-22-91$ & 464 & 7.5 & 8.5 & 03S05E10CCCB01 \\
\hline$* 60$ & 20 & 2.5 & $\mathrm{D}$ & $\mathrm{D}$ & - & -- & -- & -- & 03S05E11DABD01 \\
\hline 210.13 & 15 & -- & D & D & $06-22-91$ & 219 & 7.3 & 7.5 & 03S05E15DCCA01 \\
\hline 24.71 & 5 & -- & D & $\mathrm{D}$ & $06-18-91$ & 135 & 6.7 & 7.5 & 04S04E18ACAA01 \\
\hline & & & & & lopper Va & & & & \\
\hline 7.85 & 12 & 2.4 & $\mathrm{D}$ & D & -- & -- & -- & -- & 04S12W29CCCA01 \\
\hline 15.17 & 14 & .7 & D & D & $10-08-91$ & 83 & -- & 8.5 & 05S12W08AADC01 \\
\hline 29.03 & 11 & 1.3 & $\mathbf{S}$ & D & $10-08-91$ & 103 & -- & 7.5 & 05S12W08CBAA01 \\
\hline 150.20 & 28 & 1.0 & D & D & $10-08-91$ & 112 & -- & 10.0 & 05S12W17CBBA01 \\
\hline 22.63 & 9 & .4 & $\mathbf{S}$ & $\mathbf{D}$ & $10-08-91$ & 207 & - & 6.5 & 05S12W29BCDC01 \\
\hline
\end{tabular}


Table 1. Records of selected wells by basin, Northern Rocky Mountains intermontane basins, Montana (Continued)

\begin{tabular}{|c|c|c|c|c|c|c|c|c|c|}
\hline Woll number & $\begin{array}{l}\text { Altitude } \\
\text { of land } \\
\text { surface } \\
\text { (ft) }\end{array}$ & $\begin{array}{l}\text { Date well } \\
\text { constructed }\end{array}$ & $\begin{array}{c}\text { Geologic } \\
\text { unit }\end{array}$ & $\begin{array}{c}\text { Depth } \\
\text { of } \\
\text { woll } \\
\text { (ft) }\end{array}$ & $\begin{array}{c}\text { Dlam- } \\
\text { oter } \\
\text { of } \\
\text { casing } \\
\text { (in.) }\end{array}$ & $\begin{array}{l}\text { Type } \\
\text { of } \\
\text { finlsh }\end{array}$ & $\begin{array}{l}\text { Top of } \\
\text { open } \\
\text { Inter- } \\
\text { val } \\
\text { (ft) }\end{array}$ & $\begin{array}{c}\text { Primary } \\
\text { use of } \\
\text { water }\end{array}$ & $\begin{array}{c}\text { Date water } \\
\text { lovel } \\
\text { measured }\end{array}$ \\
\hline \multicolumn{10}{|c|}{ Grasshopper Valley--Continued } \\
\hline 05S12W31DDDD01 & 6,280 & -- & Qal & 57 & 4.0 & - & -- & $\mathbf{H}$ & $10-09-91$ \\
\hline 06S13W03DCAA01 & 6,600 & -- & QTsu & 44 & 4.0 & - & -- & s & $10-09-91$ \\
\hline $06 \mathrm{~S} 12 \mathrm{~W} 31 \mathrm{ABBA01}$ & 6,100 & $10-09-84$ & Qal & 90 & 6.0 & $\mathbf{P}$ & 70 & $\mathbf{S}$ & $10-08-91$ \\
\hline 07S 12 W06DACB01 & 6,155 & 03-30-89 & QTsu & 121 & 6.0 & $\mathbf{P}$ & 115 & $\mathbf{s}$ & $10-08-91$ \\
\hline 07S 12W 14CDDD01 & 5,950 & $11-17-73$ & Ts & 81 & 6.0 & $\mathbf{P}$ & 63 & $\mathbf{S}$ & $10-09-91$ \\
\hline 07S12W 14DCCC01 & 5,950 & $\ldots$ & Ts & 80 & -- & - & -- & $\mathbf{H}$ & $10-09-91$ \\
\hline $07 \mathrm{~S} 12 \mathrm{~W} 17 \mathrm{CDBC} 01$ & 6,120 & $11-23-74$ & Ts & 81 & 6.0 & $\mathbf{P}$ & 65 & $\mathrm{U}$ & $10-08-91$ \\
\hline $07 \mathrm{~S} 12 \mathrm{~W} 22 \wedge \mathrm{BBB} 01$ & 5,990 & $09-25-70$ & Ts & 200 & 6.0 & $\mathbf{P}$ & 195 & $\mathbf{H}$ & $10-10-91$ \\
\hline $07 \mathrm{~S} 12 \mathrm{~W} 22 \mathrm{ABCC} 01$ & 6,000 & 09-29-70 & Ts & 100 & 6.0 & $\mathbf{P}$ & 80 & $\mathbf{S}$ & $10-10-91$ \\
\hline 07S12W22BAAD01 & 6,000 & $11-23-73$ & Ts & 101 & 6.0 & $\mathbf{P}$ & 53 & $\mathbf{H}$ & $10-10-91$ \\
\hline 07S11W30DADD01 & 6,330 & $11-13-71$ & Ts & 160 & 6.0 & $\mathbf{P}$ & 118 & $\mathbf{s}$ & $10-08-91$ \\
\hline 08S 12 W01DABC01 & 5,810 & $07-26-75$ & Ts & 100 & 6.0 & $\mathbf{P}$ & 20 & $\mathbf{H}$ & -- \\
\hline 08S12W07ABBC01 & 6,580 & $11-19-81$ & Ts & 525 & 8.0 & $\mathbf{P}$ & 487 & $\mathbf{H}$ & $10-07-91$ \\
\hline 08S12W23BBBA01 & 6,010 & $09-19-84$ & Ts & 205 & 5.0 & - & - & $\mathbf{S}$ & $10-07-91$ \\
\hline $08 \mathrm{~S} 12 \mathrm{~W} 23 \mathrm{BBBB} 01$ & 6,045 & $09-25-70$ & Ts & 100 & 6.0 & $\mathbf{P}$ & 72 & $\mathrm{U}$ & $10-07-91$ \\
\hline \multicolumn{10}{|c|}{ Helena Valley } \\
\hline 11N04W25DDDD01 & 3,733 & 09. - -78 & Qal & 20 & 1.0 & $T$ & 17 & $\mathrm{U}$ & $08-21-91$ \\
\hline $11 \mathrm{~N} 03 \mathrm{~W} 15 \mathrm{DCDD} 01$ & 3,667 & $09-15-78$ & Qal & 24 & 1.5 & $\mathrm{~T}$ & 20 & $\mathrm{U}$ & $08-21-91$ \\
\hline 11 N03W20BBBB01 & 3,751 & $09--78$ & Qal & 23 & 1.5 & $\dot{T}$ & 19 & $\mathrm{U}$ & $08-21-91$ \\
\hline $11 \mathrm{~N} 03 \mathrm{~W} 21 \mathrm{DDAD} 01$ & 3,663 & $09-15-78$ & Qal & 65 & 1.5 & $\mathbf{T}$ & 61 & $\mathrm{U}$ & $08-21-91$ \\
\hline IIN03W22BBCB01 & 3,672 & $08-08-78$ & Qal & 195 & 6.0 & o & - & $\mathbf{U}$ & $08-21-91$ \\
\hline $1 \mathrm{NN} 03 \mathrm{~W} 22 \mathrm{BBCB} 02$ & 3,670 & $06-04-90$ & Qal & 48 & 2.0 & $\mathbf{G}$ & 33 & $\mathrm{U}$ & $08-21-91$ \\
\hline 11 N03W30BAAA01 & 3,747 & $09-\quad-78$ & Qal & 24 & 1.5 & $\mathrm{~T}$ & 20 & $\mathrm{U}$ & $08-21-91$ \\
\hline $11 \mathrm{~N} 03 \mathrm{~W} 30 \mathrm{BBBC} 01$ & 3,765 & -- & Qal & 127 & 10.0 & - & $\ldots$ & I & 01-08-90 \\
\hline 11N03W30DADA01 & 3,710 & $09-\quad-78$ & Qal & 44 & 1.5 & $\mathrm{~T}$ & 40 & $\mathrm{U}$ & $08-21-91$ \\
\hline 11N03W31BADD01 & 3,716 & 04-06-79 & Qal & 160 & 6.0 & $\mathbf{P}$ & 43 & $\mathrm{U}$ & $06-13-91$ \\
\hline 11N03W31BBDB01 & 3,717 & $06-10-87$ & Oal & 105 & 16.0 & $\mathbf{P}$ & 14 & $\mathrm{U}$ & $06-13-91$ \\
\hline IIN03W31DABA01 & 3,711 & $09-\quad-78$ & Qal & 24 & 1.5 & $\mathrm{~T}$ & 20 & $\mathbf{U}$ & $06-13-91$ \\
\hline IIN03W33BBAA01 & 3,678 & $07-09-90$ & Qal & 60 & 2.0 & $\mathbf{p}$ & 55 & $\mathbf{U}$ & $06-13-91$ \\
\hline 11N03W33BBAA02 & 3,679 & $07-10-90$ & Qal & 25 & 2.0 & $\mathbf{P}$ & 20 & $\mathrm{U}$ & $06-13-91$ \\
\hline 11 N03W33DDDC01 & 3,683 & $05-09-90$ & Qal & 58 & 2.0 & $\mathbf{P}$ & 53 & U & $08-21-91$ \\
\hline $11 \mathrm{~N} 03 \mathrm{~W} 33 \mathrm{DDDC0} 2$ & 3,682 & $05-08-90$ & Qal & 29 & 4.0 & $\mathbf{s}$ & 19 & $\mathbf{u}$ & $08-21-91$ \\
\hline IIN03W35DACCOI & 3,678 & $05-16-90$ & Qal & 29 & 4.0 & S & 19 & $\mathrm{U}$ & $08-21-91$ \\
\hline 11 N03W35DDBB01 & 3,679 & $05-15-90$ & Oal & 103 & 2.0 & $\mathbf{S}$ & 98 & $\mathrm{U}$ & $08-21-91$ \\
\hline 11 N02W30DCAD01 & 3,693 & $-\quad-78$ & Qal & 45 & 1.5 & $\mathbf{T}$ & 41 & $\mathbf{U}$ & $08-21-91$ \\
\hline $11 \mathrm{~N} 02 \mathrm{~W} 31 \mathrm{ACAA} 01$ & 3,700 & $-\quad-78$ & Qal & 44 & 1.5 & $T$ & 40 & $\mathrm{U}$ & $08-21-91$ \\
\hline
\end{tabular}


Table 1. Records of selected wells by basin, Northern Rocky Mountains intermontane basins, Montana (Continued)

\begin{tabular}{|c|c|c|c|c|c|c|c|c|c|}
\hline $\begin{array}{c}\text { Water level } \\
\text { (ft) }\end{array}$ & $\begin{array}{l}\text { Dis- } \\
\text { charge } \\
\text { (gsil/ } \\
\text { min) }\end{array}$ & $\begin{array}{c}\text { Spscific } \\
\text { capacity } \\
\text { [(gal/ } \\
\text { min)/fi] }\end{array}$ & $\begin{array}{l}\text { Source } \\
\text { of dis- } \\
\text { charge } \\
\text { data }\end{array}$ & $\begin{array}{l}\text { Type } \\
\text { of log } \\
\text { avali- } \\
\text { able }\end{array}$ & $\begin{array}{c}\text { Date } \\
\text { woter- } \\
\text { quallty } \\
\text { paramater } \\
\text { measured }\end{array}$ & $\begin{array}{l}\text { Speclfic } \\
\text { conduc- } \\
\text { tance } \\
(\mu S / \mathrm{cm})\end{array}$ & $\begin{array}{l}\text { pH } \\
\text { (olan- } \\
\text { dard } \\
\text { unita) }\end{array}$ & $\begin{array}{l}\text { Tom- } \\
\text { per- } \\
\text { ature } \\
\left({ }^{\circ} \mathrm{C}\right)\end{array}$ & Woli number \\
\hline \multicolumn{10}{|c|}{ Grasshopper Valley-Continued } \\
\hline 20.36 & .- & -. & - & - & $10-09-91$ & 217 & -- & 9.0 & 05S12W31DDDD01 \\
\hline 27.03 & 15 & .9 & $\mathbf{S}$ & - & $10-09-91$ & 133 & -- & 8.5 & 06S13W03DCAA01 \\
\hline 23.87 & 15 & .4 & D & D & $10-08-91$ & 359 & -- & 7.5 & 06S12W31ABBA01 \\
\hline 102.96 & 24 & - & $\mathrm{D}$ & D & -- & -- & -. & - & 07S12W06DACB01 \\
\hline 16.13 & 40 & 13 & $\mathrm{D}$ & D & $10-09-91$ & 957 & - & 7.0 & 07S12W14CDDD01 \\
\hline 26.22 & -- & -- & - & - & $10-09-91$ & 503 & -- & 10.5 & 07S12W14DCCC01 \\
\hline 49.22 & 20 & -- & $\mathbf{D}$ & D & -- & -- & -- & -. & 07S12W17CDBC01 \\
\hline 20.42 & 40 & .7 & $\mathrm{D}$ & D & $10-10-91$ & 232 & -- & 9.0 & 07S $12 \mathrm{~W} 22 \mathrm{ABBB} 01$ \\
\hline 53.90 & 30 & 1.3 & $\mathrm{D}$ & D & $10-10-91$ & 164 & - & 7.5 & $07 \mathrm{~S} 12 \mathrm{~W} 22 \mathrm{ABCC} 01$ \\
\hline+.10 & 25 & 1.5 & $\mathbf{D}$ & D & $10-10-91$ & 125 & - & 7.5 & 07S12W22BAAD01 \\
\hline 99.74 & 10 & .3 & $\mathrm{D}$ & D & -- & - & -- & -- & 07S1IW30DADD01 \\
\hline$\ldots$ & 10 & .1 & D & D & $10-07-91$ & 697 & - & 12.0 & 08S12W01DABC01 \\
\hline 425.24 & 11 & .6 & D & D & -- & $\ldots$ & -- & -- & 08S12W07ABBC01 \\
\hline 51.98 & 120 & -- & $\mathrm{D}$ & D & .- & -- & -- & -- & 08S12W23BBBA01 \\
\hline 73.37 & 20 & -- & $\mathrm{D}$ & D & -- & -- & -- & -- & 08S12W23BBBB01 \\
\hline \multicolumn{10}{|c|}{ Helena Valley } \\
\hline 4.60 & -- & -- & - & - & $08-22-88$ & 400 & -- & 20.0 & 1IN04W25DDDD01 \\
\hline 5.39 & -- & -- & - & - & $08-05-88$ & 680 & -- & 13.0 & 11N03W1 5DCDD01 \\
\hline 15.43 & -- & -. & - & - & $08-08-88$ & 320 & -- & 18.0 & $11 \mathrm{~N} 03$ W20BBBB01 \\
\hline 5.29 & -- & -- & - & - & $08-18-88$ & 510 & .. & 8.5 & 11N03W21DDAD01 \\
\hline+39.98 & 28 & .3 & $\mathbf{S}$ & D & $08-07-90$ & 440 & 7.7 & 10.0 & $11 \mathrm{~N} 03 \mathrm{~W} 22 \mathrm{BBCB} 01$ \\
\hline 10.02 & 2 & 1.9 & $\mathbf{S}$ & - & $08-07-90$ & 1,790 & 7.5 & 10.0 & 11 N03W22BBCB02 \\
\hline 17.85 & -- & -- & - & - & $08-10-88$ & 445 & -- & 15.0 & IIN03W30BAAA01 \\
\hline 39.17 & 260 & 33 & $\mathbf{S}$ & - & $05-01-80$ & 480 & -- & 10.5 & IIN03W30BBBC01 \\
\hline 1.24 & -- & -- & - & - & 08-04-88 & 105 & -- & 23.0 & 11 N03W30DADA01 \\
\hline 5.30 & 500 & - & D & D & -- & -- & -- & -- & 1IN03W3 IBADD01 \\
\hline 4.20 & .- & .- & - & D & -- & -- & -- & -- & 11N03W3 1BBDB01 \\
\hline 6.50 & .. & -- & - & - & $08-08-88$ & 530 & -- & 12.0 & IIN03W3 IDABA0I \\
\hline 3.10 & 15 & -- & $\mathbf{S}$ & - & -- & -- & -- & -- & 11N03W33BBAA01 \\
\hline 3.33 & 15 & .. & $\mathbf{s}$ & - & $08-16-90$ & 315 & 6.8 & 9.0 & 11 N03W33BBAA02 \\
\hline .83 & 15 & -- & $\mathbf{S}$ & - & $05-25-90$ & 360 & 7.3 & 10.0 & 11N03W33DDDC01 \\
\hline 1.60 & 60 & 4.7 & $\mathbf{s}$ & - & $08-06-90$ & 366 & 7.3 & 8.0 & 11N03W33DDDC02 \\
\hline 1.39 & 60 & 4.5 & $\mathbf{S}$ & - & $08-06-90$ & 897 & 7.2 & 9.0 & 11N03W35DACC01 \\
\hline+3.77 & 2 & -- & $\mathbf{S}$ & - & $08-06-90$ & 900 & 7.2 & 11.0 & 11 N03W35DDBB01 \\
\hline 26.00 & - & -- & - & - & 08-08-88 & 410 & -- & 10.0 & 11N02W3ODCADO1 \\
\hline 24.98 & -- & -- & - & - & $08-08-88$ & 330 & -- & 13.0 & IIN02W31ACAA01 \\
\hline
\end{tabular}


Table 1. Records of selected wells by basin, Northern Rocky Mountains intermontane basins, Montana (Continued)

\begin{tabular}{|c|c|c|c|c|c|c|c|c|c|}
\hline Well number & $\begin{array}{l}\text { Altitude } \\
\text { of land } \\
\text { surface } \\
\text { (ft) }\end{array}$ & $\begin{array}{l}\text { Date woll } \\
\text { constructed }\end{array}$ & $\begin{array}{c}\text { Geologic } \\
\text { unit }\end{array}$ & $\begin{array}{c}\text { Depth } \\
\text { of } \\
\text { well } \\
\text { (ft) }\end{array}$ & $\begin{array}{c}\text { Diam- } \\
\text { eter } \\
\text { of } \\
\text { casing } \\
\text { (in.) }\end{array}$ & $\begin{array}{c}\text { Type } \\
\text { of } \\
\text { finieh }\end{array}$ & $\begin{array}{l}\text { Top of } \\
\text { open } \\
\text { Intor- } \\
\text { val } \\
\text { (ft) }\end{array}$ & $\begin{array}{l}\text { Primary } \\
\text { uee of } \\
\text { water }\end{array}$ & $\begin{array}{c}\text { Date water } \\
\text { level } \\
\text { measured }\end{array}$ \\
\hline \multicolumn{10}{|c|}{ Helena Valley-Continued } \\
\hline 10N04W02CBAA01 & 4,049 & $08-08-76$ & TKi & 110 & 6.0 & $\mathbf{S}$ & 80 & $\mathbf{H}$ & $08-30-91$ \\
\hline 10N04W10DDDA01 & 3,914 & $09-15-78$ & Qal & 23 & 1.5 & $\mathbf{T}$ & 19 & $\mathbf{U}$ & $08-21-91$ \\
\hline 10N04W 12ACDA01 & 3,799 & $09-15-78$ & Qal & 23 & 1.5 & $T$ & 19 & $\mathbf{U}$ & $08-21-91$ \\
\hline 10N04W 12CADB01 & 3,830 & $01-01-78$ & Qal & 23 & 1.5 & $\mathbf{T}$ & 19 & $\mathrm{U}$ & $08-21-91$ \\
\hline 10N04W 13ACCD01 & 3,831 & $09-30-74$ & Qal & 19 & 6.0 & $\mathbf{O}$ & - & $\mathbf{U}$ & $08-21-91$ \\
\hline 10N04W 13ACCD02 & 3,832 & $08-21-84$ & Qal & 93 & 6.0 & $\mathrm{o}$ & -- & H & $06-13-91$ \\
\hline 10N04W15BAAB01 & 3,949 & $11-16-83$ & TKi & 150 & 10.0 & $\mathbf{P}$ & 34 & I & $06-13-91$ \\
\hline 10N04W 15BABD01 & 3,953 & $11-16-83$ & TKi & 250 & 10.0 & $\mathbf{P}$ & 44 & I & $06-13-91$ \\
\hline 10N04W15CCCCO1 & 4,012 & $11-16-81$ & Qal & 135 & 8.0 & $\mathbf{P}$ & 30 & $\mathbf{U}$ & $08-21-91$ \\
\hline 10N04W 15DBBB01 & 3,943 & $09-15-78$ & Qal & 35 & 1.5 & $\mathbf{T}$ & 31 & U & $08-21-91$ \\
\hline 10N04W 23ABBC01 & 3,900 & 09-02-88 & Qal & 60 & 6.0 & o & -- & $\mathbf{U}$ & $06-13-91$ \\
\hline 10N04W23BAAB01 & 3,915 & -- & Qal & 500 & 6.0 & & -- & $\mathbf{U}$ & $05-24-90$ \\
\hline 10N04W23BBBB01 & 3,915 & $09-15-78$ & Qal & 23 & 1.5 & $T$ & 19 & $\mathbf{U}$ & $08-21-91$ \\
\hline 10N04W23BBCB01 & 3,919 & $-\quad-87$ & Qal & 60 & 6.0 & $\mathbf{P}$ & 50 & $\mathbf{U}$ & $08-21-91$ \\
\hline 10N04W23BBCB02 & 3,919 & $06-05-90$ & Qal & 28 & 2.0 & $\mathbf{S}$ & 23 & U & $08-21-91$ \\
\hline 10N03W02DDDD02 & 3,720 & $07-13-90$ & Qal & 104 & 2.0 & $\mathrm{~s}$ & 99 & $\mathbf{U}$ & $08-21-91$ \\
\hline 10N03W02DDDD03 & 3,720 & $07-13-90$ & Qal & 25 & 2.0 & $\mathbf{S}$ & 20 & $\mathbf{U}$ & $08-21-91$ \\
\hline 10N03W03BACB01 & 3,684 & $09 \ldots$ & Qal & 65 & 1.5 & $\mathbf{T}$ & 61 & $\mathbf{U}$ & $09-20-91$ \\
\hline 10N03W03BACB02 & 3,683 & 09- -78 & Qal & 24 & 1.5 & $\mathbf{T}$ & 21 & $\mathrm{u}$ & $06-13-91$ \\
\hline 10N03W04DCCD01 & 3,704 & $07-17-90$ & Qal & 55 & 4.0 & $\mathbf{P}$ & 45 & U & $06-13-91$ \\
\hline 10N03W04DCCD02 & 3,704 & $07-18-90$ & Qal & 25 & 4.0 & $\mathbf{P}$ & 15 & $\mathbf{U}$ & $06-13-91$ \\
\hline 10N03W05BAAA01 & 3,714 & $09-\quad-78$ & Qal & 65 & 1.5 & $\mathbf{T}$ & 61 & $\mathrm{U}$ & $08-21-91$ \\
\hline 10N03W05BAAB01 & 3,715 & $09-\quad-78$ & Qal & 28 & 1.5 & $\mathbf{T}$ & 24 & $\mathbf{U}$ & $08-21-91$ \\
\hline 10N03W05CCDD01 & 3,743 & $09--78$ & Qal & 23 & 1.5 & $\mathbf{T}$ & 19 & $\mathbf{U}$ & $08-21-91$ \\
\hline 10N03W06ABBC01 & 3,735 & $09-01-78$ & Qal & 62 & 1.5 & $\mathbf{T}$ & 58 & U & $08-19-88$ \\
\hline 10N03W06DBAA01 & 3,747 & $09--78$ & Qal & 34 & 1.5 & $T$ & 30 & $\mathbf{U}$ & $08-21-91$ \\
\hline 10N03W06DBAA02 & 3,747 & $09--78$ & Qal & 62 & 1.5 & $\mathbf{T}$ & 58 & $\mathbf{U}$ & $08-21-91$ \\
\hline 10N03W07AAAA01 & 3,751 & -- & Qal & -- & 6.0 & - & -- & $\mathbf{U}$ & $06-13-91$ \\
\hline 10N03W08CBCC01 & 3,767 & $09-\quad-78$ & Qal & 23 & 1.5 & $\mathbf{T}$ & 19 & $\mathbf{U}$ & $06-13-91$ \\
\hline 10N03W09ACCCOI & 3,713 & 09- -78 & Qal & 65 & 1.5 & $\mathbf{T}$ & 21 & $\mathbf{U}$ & $09-20-91$ \\
\hline 10N03W09ACCC02 & 3,713 & $09-01-78$ & Qal & 22 & 1.5 & $\mathbf{T}$ & 63 & $\mathbf{u}$ & $06-13-91$ \\
\hline 10N03W11ABBB01 & 3,717 & --78 & Qal & 24 & 1.5 & $\mathbf{T}$ & 20 & $\mathbf{U}$ & $08-21-91$ \\
\hline 10N03W IIDDCC01 & 3,752 & $09--78$ & Qal & 40 & 1.5 & $\mathbf{T}$ & 40 & $\mathbf{U}$ & $08-21-91$ \\
\hline 10N03W $11 \mathrm{DDCC} 02$ & 3,753 & $06-07-90$ & Qal & 78 & 2.0 & G & 73 & $\mathbf{U}$ & $08-21-91$ \\
\hline 10N03W13BADD01 & 3,766 & $10-15-83$ & Qal & 188 & 8.0 & o & -- & $\mathbf{U}$ & $08-21-91$ \\
\hline
\end{tabular}


Table 1. Records of selected wells by basin, Northern Rocky Mountains intermontane basins, Montana (Continued)

\begin{tabular}{|c|c|c|c|c|c|c|c|c|c|}
\hline $\begin{array}{l}\text { Water level } \\
\text { (ft) }\end{array}$ & $\begin{array}{c}\text { Dis- } \\
\text { charge } \\
\text { (gal/ } \\
\text { min) }\end{array}$ & $\begin{array}{c}\text { Specific } \\
\text { capacity } \\
\text { [(gal/ } \\
\text { min)/ft] }\end{array}$ & $\begin{array}{c}\text { Source } \\
\text { of dis- } \\
\text { charge } \\
\text { dala }\end{array}$ & $\begin{array}{l}\text { Type } \\
\text { of log } \\
\text { ovali- } \\
\text { able }\end{array}$ & $\begin{array}{c}\text { Date } \\
\text { water- } \\
\text { quality } \\
\text { parameter } \\
\text { measured }\end{array}$ & $\begin{array}{c}\text { Specific } \\
\text { conduc- } \\
\text { tance } \\
(\mu \mathrm{S} / \mathrm{cm})\end{array}$ & $\begin{array}{l}\mathrm{pH} \\
\text { (stan- } \\
\text { dand } \\
\text { units) }\end{array}$ & $\begin{array}{l}\text { Tom- } \\
\text { per- } \\
\text { ature } \\
\left({ }^{\circ} \mathrm{C}\right)\end{array}$ & Woll number \\
\hline
\end{tabular}

\begin{tabular}{|c|c|c|c|c|c|c|c|c|c|}
\hline 36.57 & 30 & .5 & D & D & $05-31-90$ & 450 & 7.6 & 11.0 & 10N04W02CBAA01 \\
\hline 7.47 & -- & -- & - & - & $08-11-88$ & 380 & .. & 13.5 & 10N04W10DDDA01 \\
\hline 8.12 & -- & -- & - & - & $08-11-88$ & 520 & -- & 15.0 & 10N04W12ACDA01 \\
\hline 14.19 & .. & -- & - & - & $08-11-88$ & 535 & -- & 10.5 & 10N04W12CADB01 \\
\hline 4.03 & 2 & 17 & $\mathbf{s}$ & - & $01-23-91$ & 460 & 7.5 & 8.0 & 10N04W13ACCD01 \\
\hline 32.50 & 35 & 1.3 & L & - & $11-07-90$ & 300 & 7.6 & 8.0 & 10N04W13ACCD02 \\
\hline 26.36 & 280 & 21 & L & D & -- & -- & -- & -- & 10N04W15BAAB01 \\
\hline 33.38 & 250 & 38 & L & - & -- & - & -- & - & 10N04W15BABD01 \\
\hline 52.16 & 23 & .4 & D & D & - & - & - & -- & 10N04W15CCCC01 \\
\hline 21.24 & -. & -- & - & - & $08-11-88$ & 620 & -- & 12.5 & 10N04W15DBBB01 \\
\hline 2.20 & 30 & -. & $\mathbf{L}$ & D & -- & -- & -- & -- & 10N04W23ABBC01 \\
\hline 6.90 & -- & -- & - & - & -- & - & -- & -- & 10N04W23BAAB01 \\
\hline 4.69 & - & -- & - & - & $08-05-88$ & 260 & -- & 11.5 & 10N04W23BBBB01 \\
\hline 6.60 & -- & -- & - & - & 08-02-90 & 315 & 7.0 & 10.0 & 10N04W23BBCB01 \\
\hline 6.64 & 2 & -- & $\mathbf{s}$ & - & $08-02-90$ & 249 & 7.1 & 10.5 & 10N04W23BBCB02 \\
\hline 12.00 & 1 & -. & $\mathbf{s}$ & - & -- & -- & - & -- & 10N03W02DDDD02 \\
\hline 10.37 & 10 & -- & $\mathbf{s}$ & - & 08-14-90 & 480 & 7.1 & 12.0 & 10N03W02DDDD03 \\
\hline 2.44 & -- & -- & - & - & $08-17-88$ & 385 & -- & 9.0 & 10N03W03BACB01 \\
\hline 2.77 & -- & -- & - & - & $08-17-88$ & 590 & - & 8.5 & 10N03 W03BACB02 \\
\hline 1.62 & 20 & -- & $\mathbf{s}$ & - & 08-07-90 & 462 & 7.5 & 9.5 & 10N03W04DCCD01 \\
\hline 4.88 & 20 & 4.5 & $\mathbf{s}$ & - & $08-07-90$ & 641 & 7.5 & 8.5 & 10N03W04DCCD02 \\
\hline 9.38 & - & -- & - & - & $08-17-88$ & 550 & -- & 10.5 & 10N03W05BAAA01 \\
\hline 8.98 & -- & -- & - & - & 08-17-88 & 575 & -- & 10.5 & 10N03W05BAAB01 \\
\hline 10.55 & -- & -- & - & - & $09-25-90$ & 468 & 7.4 & 9.0 & 10N03W05CCDD01 \\
\hline 17.87 & - & -- & $\cdot$ & - & $08-19-88$ & 590 & -- & 11.5 & 10N03W06ABBC01 \\
\hline 19.98 & - & -- & - & - & $11-03-90$ & 540 & 7.0 & 8.0 & 10N03W06DBAA01 \\
\hline 19.90 & -- & -- & - & - & $11-03-90$ & 407 & 6.9 & 9.0 & 10N03W06DBAA02 \\
\hline 16.84 & -- & -- & - & - & -- & -- & -- & -- & 10N03W07AAAA01 \\
\hline 14.55 & -- & -- & - & - & $08-16-88$ & 465 & -- & 11.0 & 10N03W08CBCC01 \\
\hline 2.37 & -- & -- & - & - & 08-16-88 & 520 & - & 13.0 & 10N03W09ACCC01 \\
\hline 3.86 & -- & -- & - & - & $08-16-88$ & 650 & -- & 12.5 & 10N03W09ACCC02 \\
\hline 5.57 & -- & -- & - & - & $08-10-88$ & 280 & -- & 14.5 & 10N03W11ABBB01 \\
\hline 17.62 & - & -- & - & - & $08-10-90$ & 262 & 7.6 & 15.0 & 10N03W11DDCC01 \\
\hline 18.79 & 2 & -- & $\mathbf{S}$ & - & $08-09-90$ & 415 & 7.1 & 10.5 & 10N03W11DDCC02 \\
\hline 31.30 & 280 & 2.3 & $\mathbf{L}$ & D & -- & -- & - & -- & 10N03W13BADD01 \\
\hline
\end{tabular}


Table 1. Records of selected wells by basin, Northern Rocky Mountains intermontane basins, Montana (Continued)

\begin{tabular}{|c|c|c|c|c|c|c|c|c|c|}
\hline Weli number & $\begin{array}{c}\text { Altitude } \\
\text { of land } \\
\text { surface } \\
\text { (ft) }\end{array}$ & $\begin{array}{l}\text { Dale weli } \\
\text { constructed }\end{array}$ & $\begin{array}{c}\text { Geologlc } \\
\text { unit }\end{array}$ & $\begin{array}{c}\text { Depth } \\
\text { of } \\
\text { well } \\
\text { (ft) }\end{array}$ & $\begin{array}{c}\text { Dlam- } \\
\text { otor } \\
\text { of } \\
\text { casing } \\
\text { (in.) }\end{array}$ & $\begin{array}{l}\text { Type } \\
\text { of } \\
\text { firish }\end{array}$ & $\begin{array}{l}\text { Top of } \\
\text { open } \\
\text { inter- } \\
\text { val } \\
\text { (ft) }\end{array}$ & $\begin{array}{l}\text { Primary } \\
\text { uee of } \\
\text { water }\end{array}$ & $\begin{array}{l}\text { Dato water } \\
\text { level } \\
\text { measured }\end{array}$ \\
\hline \multicolumn{10}{|c|}{ Helena Valley--Continued } \\
\hline 10N03W15BCBA01 & 3,734 & $09 .-78$ & Qal & 25 & 1.5 & $\mathbf{T}$ & 21 & $\mathbf{U}$ & $08-21-91$ \\
\hline 10N03W15DDCA01 & 3,769 & $11-13-87$ & Qal & 326 & 8.0 & $\mathbf{x}$ & 287 & $\mathbf{U}$ & $05-01-91$ \\
\hline 10N03W 15DDDD02 & 3,765 & -- & Qal & -- & -- & - & - & $\mathbf{U}$ & $11-15-83$ \\
\hline 10N03W $16 \mathrm{DBAD} 01$ & 3,756 & 09- -78 & QTsu & 44 & 1.5 & $\mathbf{T}$ & 40 & $\mathbf{U}$ & $08-21-91$ \\
\hline 10N03W17ABBB01 & 3,772 & $09-\quad-78$ & Qal & 28 & 1.5 & $T$ & 24 & $\mathbf{U}$ & $08-21-91$ \\
\hline 10N03W17ACAD01 & 3,775 & 09. -78 & Qal & 28 & 1.5 & $T$ & 24 & $\mathbf{U}$ & $09-20-91$ \\
\hline 10N03W17ACCC01 & 3,785 & $09-\quad-78$ & Qal & 32 & 1.5 & $\mathbf{T}$ & 28 & $\mathbf{U}$ & $08-21-91$ \\
\hline 10N03W17ACCCO2 & 3,785 & $09-\quad-78$ & Qal & 45 & 1.5 & $\mathbf{T}$ & 41 & $\mathbf{U}$ & $08-21-91$ \\
\hline 10N03W17CCCC01 & 3,837 & $03-05-87$ & Qal & 140 & 6.0 & $\mathbf{x}$ & 120 & $\mathbf{U}$ & $06-13-91$ \\
\hline 10N03W17DDAD01 & 3,796 & $09-\quad-78$ & Qal & 43 & 1.5 & $T$ & 39 & $\mathbf{U}$ & $12-09-91$ \\
\hline 10N03W 17DDAD02 & 3,795 & 09-01-78 & Qal & 25 & 1.5 & $T$ & 21 & $\mathbf{U}$ & $06-13-91$ \\
\hline 10N03W22AAAA01 & 3,774 & $09-\quad-78$ & Qal & 23 & 1.5 & $\mathbf{T}$ & 19 & $\mathbf{U}$ & $08-21-91$ \\
\hline 10N03W23DAAD01 & 3,816 & - & Qal & 180 & 6.0 & - & -- & $\mathbf{U}$ & $05-01-91$ \\
\hline 10N03W25CDBA01 & 3,871 & $08-04-82$ & Ts & 160 & 6.0 & $\mathbf{P}$ & 120 & $\mathbf{H}$ & $08-21-91$ \\
\hline 10N03W25CDBA02 & 3,872 & $03-08-70$ & Ts & 82 & 6.0 & o & - & $\mathbf{U}$ & $08-21-91$ \\
\hline $10 \mathrm{~N} 03 \mathrm{~W} 27 \mathrm{CCAB01}$ & 3,948 & $08-25-81$ & Ts & 110 & 6.0 & $\mathbf{S}$ & 50 & $\mathbf{H}$ & $08-21-91$ \\
\hline 10N02W06CBCC01 & 3,707 & - -78 & Qal & 24 & 1.5 & $\mathrm{~T}$ & 20 & $\mathbf{U}$ & $08-21-91$ \\
\hline 10N02W07BBBB01 & 3,717 & --78 & Qal & 24 & 1.5 & $T$ & 20 & $\mathbf{U}$ & $06-13-91$ \\
\hline 10N02W 18DDCD01 & 3,784 & $02-27-79$ & Ts & 70 & 6.6 & 0 & -- & $\mathbf{H}$ & $08-21-91$ \\
\hline \multicolumn{10}{|c|}{ Horse Prairie Valley } \\
\hline 08S12W32CDDD01 & 6,037 & -- & Ts & 125 & 6.0 & - & -. & $\mathbf{S}$ & $10-10-91$ \\
\hline 09S14W25DDCB01 & 6,156 & $12-06-90$ & Ts & 80 & 6.0 & $\mathbf{S}$ & 60 & $\mathbf{H}$ & $10-07-91$ \\
\hline 09S14W25DDCC01 & 6,152 & $11-14-80$ & Ts & 192 & 6.0 & $\mathbf{P}$ & 175 & $\mathbf{H}$ & $10-07-91$ \\
\hline 09S13W08AA 01 & 6,460 & $07-29-91$ & Ts & 160 & 6.0 & $\mathbf{P}$ & 100 & $\mathbf{H}$ & $10-09-91$ \\
\hline 09S13W14DBAB01 & 6,162 & $11-18-81$ & Ts & 180 & 8.0 & $\mathbf{P}$ & 145 & $\mathbf{S}$ & $10-09-91$ \\
\hline 09S13W15CDCC01 & 6,228 & $07-29-91$ & Ts & 145 & 6.0 & $\mathbf{P}$ & 80 & H & $10-09-91$ \\
\hline 09S13W35ABCB01 & 5,939 & $07-08-88$ & Ts & 120 & 6.0 & $\mathbf{P}$ & 60 & $\mathbf{H}$ & $10-09-91$ \\
\hline 09S12W03BDAC01 & 6,020 & $09-15-43$ & Ts & 94 & 6.5 & - & - & $\mathbf{S}$ & $10-10-91$ \\
\hline 09S12W06BBCD01 & 6,370 & $09-18-70$ & Ts & 403 & 6.0 & $\mathbf{P}$ & 293 & $\mathbf{S}$ & $10-10-91$ \\
\hline 09S12W08ACDA01 & 5,946 & $09-22-60$ & Ts & 114 & 6.0 & - & - & $\mathbf{S}$ & $10-10-91$ \\
\hline 09S 12 W11BBDD01 & 6,020 & $10-25-43$ & Ts & 200 & 4.3 & 0 & -- & $\mathbf{U}$ & $10-10-91$ \\
\hline 09S12W14ACAD01 & 5,970 & - & Ts & 275 & -- & - & -- & $\mathbf{u}$ & $10-10-91$ \\
\hline 09S12W18DDAD01 & 5,865 & $10-18-58$ & Ts & 38 & 6.0 & $\mathrm{x}$ & 38 & H & $10-09-91$ \\
\hline 09S12W18DDAD02 & 5,865 & $08-15-61$ & Ts & 46 & 6.0 & - & - & I & $10-09-91$ \\
\hline $09 \mathrm{~S} 12 \mathrm{~W} 22 \mathrm{DCBB} 01$ & 5,760 & $09-21-88$ & Qal & 40 & 6.0 & $\mathbf{P}$ & 31 & $\mathbf{S}$ & $10-08-91$ \\
\hline
\end{tabular}


Table 1. Records of selected wells by basin, Northern Rocky Mountains intermontane basins, Montana (Continued)

\begin{tabular}{|c|c|c|c|c|c|c|c|c|c|}
\hline $\begin{array}{c}\text { Water level } \\
\text { (ft) }\end{array}$ & $\begin{array}{c}\text { Dis- } \\
\text { charge } \\
\text { (gal/ } \\
\text { min) }\end{array}$ & $\begin{array}{l}\text { Specific } \\
\text { capacity } \\
\text { [(gall } \\
\text { min)/ft] }\end{array}$ & $\begin{array}{c}\text { Source } \\
\text { of dis- } \\
\text { charge } \\
\text { data }\end{array}$ & $\begin{array}{l}\text { Type } \\
\text { of log } \\
\text { avall- } \\
\text { able }\end{array}$ & $\begin{array}{c}\text { Date } \\
\text { water- } \\
\text { quallty } \\
\text { parameter } \\
\text { measured }\end{array}$ & $\begin{array}{l}\text { Specific } \\
\text { conduc- } \\
\text { tance } \\
\text { ( } \mathrm{HS} / \mathrm{cm})\end{array}$ & $\begin{array}{c}\text { pH } \\
\text { (elan- } \\
\text { dard } \\
\text { units) }\end{array}$ & $\begin{array}{l}\text { Tom- } \\
\text { per- } \\
\text { ature } \\
\left({ }^{\circ} \mathrm{C}\right)\end{array}$ & Well number \\
\hline
\end{tabular}

\begin{tabular}{|c|c|c|c|c|c|c|c|c|c|}
\hline \multicolumn{10}{|c|}{ Helena Valley--Continued } \\
\hline .40 & -- & -- & - & - & $08-10-88$ & 440 & -- & 10.5 & 10N03W1 5BCBA01 \\
\hline 12.70 & -- & -- & - & - & 08-13-90 & 250 & 7.6 & 12.5 & 10N03W15DDCA01 \\
\hline Flowing & - & -- & - & - & -- & - & -- & -- & 10N03W15DDDD02 \\
\hline 6.68 &. & -- & - & - & $08-10-88$ & 565 & -- & 11.0 & 10N03W16DBAD01 \\
\hline 19.90 & -- & -- & - & - & 08-11-88 & 365 & -- & 14.0 & 10N03W17ABBBOI \\
\hline 17.37 & -- & -- & - & - & 08-11-88 & 330 & -. & 19.0 & 10N03W17ACAD01 \\
\hline 25.68 & -- & -- & - & - & $08-11-88$ & 405 & .. & 13.0 & $10 \mathrm{~N} 03 \mathrm{~W} 17 \mathrm{ACCC} 01$ \\
\hline 25.80 & -- & -- & - & - & $08-11-88$ & 425 & -- & 11.5 & 10N03W17ACCCO2 \\
\hline 1.40 & 30 & -- & D & D & -- & -- & -- & -- & 10N03W17CCCC01 \\
\hline Flowing & -- & -- & $\cdot$ & - & $08-12-88$ & 375 & -- & 10.0 & 10N03W17DDAD01 \\
\hline 24.21 & - & -- & - & - & -- & - & - & - & 10N03W17DDAD02 \\
\hline 13.42 & - & -- & - & - & 08-14-90 & 192 & 7.8 & 9.0 & 10N03W22AAAA01 \\
\hline 46.62 & - & -- & - & - & $08-09-90$ & 325 & 7.2 & 10.0 & 10N03W23DAAD01 \\
\hline 51.82 & 20 & .2 & D & D & $08-08-90$ & 575 & 7.1 & 11.5 & 10N03W25CDBA01 \\
\hline 7.80 & 1 & -- & $\mathbf{S}$ & D & $08-13-90$ & 1,320 & 6.9 & 14.5 & 10N03W25CDBA02 \\
\hline 26.44 & 30 & .4 & D & D & $08-16-90$ & 750 & 7.4 & 11.5 & 10N03W27CCAB01 \\
\hline 5.20 & -- & -- & - & - & $08-18-88$ & 410 & -- & 20.0 & $10 \mathrm{~N} 02 \mathrm{~W} 06 \mathrm{CBCC} 01$ \\
\hline 10.60 & -- & -. & - & - & $08-17-90$ & 390 & 6.6 & 17.0 & 10N02W07BBBB01 \\
\hline 46.35 & 15 & 4.8 & D & D & $06-14-83$ & 420 & 7.5 & 9.5 & 10N02W18DDCD01 \\
\hline \multicolumn{10}{|c|}{ Horse Prairie Valley } \\
\hline 53.80 & 10 & .2 & D & - & -- & - & -- & -- & 08S12W32CDDD01 \\
\hline 29.05 & 30 & .9 & D & D & $10-07-91$ & 508 & 8.2 & 12.0 & 09S14W25DDCB01 \\
\hline 23.57 & 6 & 7.1 & 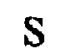 & D & $10-07-91$ & 562 & 8.6 & 8.0 & 09S14W25DDCC01 \\
\hline 37.25 & 40 & -- & D & D & -- & -- & -- & -- & 09S13W08AA 01 \\
\hline 75.89 & 17 & .4 & D & D & -- & -- & - & - & O9S13W 14DBAB01 \\
\hline 29.36 & 30 & .3 & D & D & -- & -. & -- & - & 09S13W15CDCC01 \\
\hline 5.55 & 8 & .8 & $\mathbf{s}$ & $\mathbf{D}$ & $10-09-91$ & 369 & 8.4 & 7.0 & 09S13W35ABCB01 \\
\hline 67.10 & 10 & 2.5 & D & D & -- & -- & -- & -- & 09S12W03BDAC0I \\
\hline 286.70 & 12 & .5 & D & D & -- & -- & -- & -- & 09S12W06BBCD01 \\
\hline 62.82 & 20 & .6 & D & D & -- & -- & - & - & 09S12W08ACDA01 \\
\hline$* 176$ & 5 & .6 & D & D & -- & -- & -- & -- & 09S12W11BBDD01 \\
\hline 226.81 & -- & -- & - & - & -- & -- & -- & -. & 09S12W14ACAD01 \\
\hline 19.48 & 25 & 2.3 & D & D & $10-09-91$ & 613 & 7.5 & 9.0 & 09S12W18DDAD01 \\
\hline 17.90 & 30 & 2.1 & D & D & -- & -- & - & -- & 09S 12W18DDAD02 \\
\hline 3.95 & 40 & 2.0 & D & D & $10-08-91$ & 321 & 6.9 & 6.0 & 09S12W22DCBB01 \\
\hline
\end{tabular}


Table 1. Records of selected wells by basin, Northern Rocky Mountains intermontane basins, Montana (Continued)

\begin{tabular}{|c|c|c|c|c|c|c|c|c|c|}
\hline Well number & $\begin{array}{l}\text { Altitude } \\
\text { of land } \\
\text { surface } \\
\text { (ft) }\end{array}$ & $\begin{array}{l}\text { Date well } \\
\text { constructed }\end{array}$ & $\begin{array}{c}\text { Geologic } \\
\text { unit }\end{array}$ & $\begin{array}{l}\text { Depth } \\
\text { of } \\
\text { well } \\
\text { (fi) }\end{array}$ & $\begin{array}{c}\text { Dlam- } \\
\text { eter } \\
\text { of } \\
\text { casing } \\
\text { (In.) }\end{array}$ & $\begin{array}{c}\text { Type } \\
\text { of } \\
\text { finlsh }\end{array}$ & $\begin{array}{l}\text { Top of } \\
\text { open } \\
\text { Intar- } \\
\text { val } \\
\text { (ft) }\end{array}$ & $\begin{array}{c}\text { Primary } \\
\text { use of } \\
\text { water }\end{array}$ & $\begin{array}{l}\text { Date water } \\
\text { level } \\
\text { measured }\end{array}$ \\
\hline \multicolumn{10}{|c|}{ Horse Prairie Valley-Continued } \\
\hline 09S12W22DCBD01 & 5,758 & $05-04-83$ & Ts & 162 & 6.0 & 0 & -- & $\mathbf{H}$ & $10-08-91$ \\
\hline 09S12W22DCBD02 & 5,758 & $06-30-82$ & Qal & 80 & 6.0 & 0 & -- & $\mathrm{U}$ & $10-08-91$ \\
\hline 09S12W29CBBB01 & 5,855 & -- & Qal & 34 & -- & - & -- & $\mathrm{H}$ & $10-08-91$ \\
\hline 09S12W33DAAA01 & 5,810 & $12-05-90$ & Qal & 50 & 6.0 & $\mathbf{P}$ & 40 & $\mathbf{H}$ & $10-09-91$ \\
\hline 09S12W34CBAB01 & 5,810 & -- & QTsu & 76 & 5.0 & - & -- & $\mathbf{H}$ & $10-08-91$ \\
\hline 10S14W03BBCB01 & 6,293 & $08-24-71$ & Ts & 40 & 6.0 & - & -- & $\mathbf{H}$ & $10-07-91$ \\
\hline 10S14W 10BDAD01 & 6,279 & $09-28-67$ & Ts & 37 & 6.0 & - & -- & $\mathbf{S}$ & $10-09-91$ \\
\hline $10 \mathrm{~S} 14 \mathrm{~W} 23 \mathrm{CDBB} 01$ & 6,425 & $03-28-68$ & Ts & 243 & 6.0 & $\mathbf{P}$ & 235 & $\mathrm{U}$ & $10-07-91$ \\
\hline 10S13W03BBAA01 & 6,041 & $03-19-74$ & Ts & 135 & 6.0 & $\mathbf{P}$ & 122 & H & $10-09-91$ \\
\hline $10 S 12 \mathrm{~W} 11 \mathrm{AABD} 01$ & 5,820 & $10-27-88$ & Ts & 180 & 5.0 & $\mathbf{P}$ & 140 & $\mathbf{U}$ & $10-10-91$ \\
\hline 10S12W25AACB01 & 5,890 & $02-22-83$ & QTsu & 160 & 6.0 & $\mathbf{P}$ & 139 & $\mathbf{s}$ & $10-08-91$ \\
\hline 10S12W27CADA01 & 6,228 & $10-10-79$ & Ts & 200 & 6.0 & $\mathbf{P}$ & 140 & $\mathbf{U}$ & $10-08-91$ \\
\hline IOSIIWO8BCBA01 & 5,700 & $10-31-74$ & Qal & 50 & 6.0 & $\mathbf{P}$ & 38 & $\mathbf{S}$ & $10-10-91$ \\
\hline \multicolumn{10}{|c|}{ Jefferson River Valley } \\
\hline 03N04W21ABDB01 & 4,770 & $04-06-89$ & Ts & 78 & 6.0 & $\mathbf{P}$ & 70 & U & $06-19-91$ \\
\hline 02N05W27CBCB0I & 4,555 & $03-30-86$ & Ts & 75 & 6.0 & o & -- & H & $06-20-91$ \\
\hline 02N04W04DCCC01 & 4,640 & $09-10-83$ & Ts & 120 & 6.0 & 0 & -- & $\mathbf{H}$ & $06-19-91$ \\
\hline 02N04W28CDCB01 & 4,415 & $12-20-89$ & Qal & 80 & 6.0 & o & -- & $\mathbf{H}$ & $06-20-91$ \\
\hline 02N03W33DCD 01 & 4,350 & -- & Ts & 67 & -- & - & -- & $\mathrm{U}$ & $06-20-91$ \\
\hline 02N03W34CCD 01 & 4,370 & -- & Ts & 220 & 6.0 & - & -- & $\mathbf{U}$ & 08-01-91 \\
\hline 01N05W03CCAC01 & 4,680 & $08-25-87$ & Ts & 83 & 6.0 & $\mathbf{P}$ & 76 & H & $06-20-91$ \\
\hline 01N05W 22AAAA01 & 4,583 & $04-05-88$ & Ts & 180 & 6.5 & $\mathbf{P}$ & 100 & $\mathrm{U}$ & $06-22-91$ \\
\hline 01N05W24DCCA01 & 4,475 & 09-16-69 & Ts & 131 & 6.0 & $\mathbf{P}$ & 121 & $\mathbf{H}$ & $06-22-91$ \\
\hline 01N04W03BADD01 & 4,355 & -- & Ts & 146 & 6.0 & - & - & $\mathrm{U}$ & $06-19-91$ \\
\hline 01N04W04ABBA01 & 4,380 & $05-04-58$ & Ts & 375 & 16.0 & $\mathbf{S}$ & 130 & $\mathbf{P}$ & -- \\
\hline 01 N04W04ACCD01 & 4,350 & $03-12-52$ & Ts & 349 & 12.0 & - & 272 & $\mathbf{P}$ & $06-19-91$ \\
\hline 01N04W04ADDA01 & 4,350 & 04-15-74 & Qal & 106 & 10.0 & $\mathbf{P}$ & 35 & $\mathbf{P}$ & -- \\
\hline 01N04W07BACA01 & 4,580 & $07-11-84$ & Ts & 302 & 6.0 & 0 & -- & H & $06-22-91$ \\
\hline 01N03W03DAA 01 & 4,285 & $07-12-67$ & Qal & 59 & 6.0 & o & - & $\mathbf{H}$ & $06-18-91$ \\
\hline 01N03W17ABDA01 & 4,460 & $06-05-72$ & Ts & 202 & 6.0 & o & -- & $\mathbf{H}$ & $06-21-91$ \\
\hline 01S05W 14BBBC01 & 4,505 & $12-22-88$ & Ts & 100 & 6.0 & o & -- & $\mathbf{H}$ & $06-20-91$ \\
\hline 01S05W26ADCC01 & 4,473 & --35 & Qal & 35 & 6.0 & - & -- & H & -- \\
\hline $01505 W 26 D C D \quad 01$ & 4,465 & $09-21-82$ & Qal & 85 & 6.0 & $\mathbf{P}$ & 12 & $\mathrm{U}$ & 06-20-91 \\
\hline 01S04W06DDBC01 & 4,435 & $08-23-71$ & Qal & 60 & 6.0 & $\mathbf{P}$ & 50 & $\mathbf{H}$ & $06-21-91$ \\
\hline
\end{tabular}


Table 1. Records of selected wells by basin, Northern Rocky Mountains intermontane basins, Montana (Continued)

\begin{tabular}{|c|c|c|c|c|c|c|c|c|c|}
\hline $\begin{array}{c}\text { Water level } \\
\text { (ft) }\end{array}$ & $\begin{array}{l}\text { Dis- } \\
\text { charge } \\
\text { (gal/ } \\
\text { min) }\end{array}$ & $\begin{array}{c}\text { Specific } \\
\text { capacity } \\
\text { [(gal/ } \\
\text { min)/ft] }\end{array}$ & $\begin{array}{l}\text { Source } \\
\text { of dis- } \\
\text { charge } \\
\text { data }\end{array}$ & $\begin{array}{l}\text { Type } \\
\text { of log } \\
\text { avait } \\
\text { able }\end{array}$ & $\begin{array}{c}\text { Date } \\
\text { water- } \\
\text { quailty } \\
\text { parameter } \\
\text { measured }\end{array}$ & $\begin{array}{c}\text { Specific } \\
\text { conduc- } \\
\text { tance } \\
(\mu \mathrm{S} / \mathrm{cm})\end{array}$ & $\begin{array}{c}\text { pH } \\
\text { (etan- } \\
\text { dard } \\
\text { units) }\end{array}$ & $\begin{array}{l}\text { Tem- } \\
\text { per- } \\
\text { ature } \\
\left({ }^{\circ} \mathrm{C}\right)\end{array}$ & Well number \\
\hline
\end{tabular}

Horse Prairie Valley-Continued

11.58

9.18
6.31

29.25

24.17

4.23

8.45

47.62

30.66

88.45

11.02

25.20

3.62

-
45
-

$\cdots$
$\cdots$
$\cdots$
$\cdots$

$\begin{array}{rc}40 & 4.4 \\ 30 & 3.0 \\ 50 & -- \\ 6 & .4 \\ 50 & .5\end{array}$

$\begin{array}{ll}11 & .5 \\ 90 & 15 \\ 12 & .7\end{array}$

D

D

D
D
-
D
-

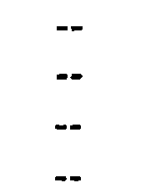

$10-08-91$

\section{D $\quad 10-07-91 \quad 248$}

D $\quad 10-09-91 \quad 285$

D $\quad--$

D 10-09-91

$--$

537

D

D 10-08-91

D --

D $\quad 10-10-91$

2,300
$\cdots$
982

\section{Jefferson River Valley}

\begin{tabular}{|c|c|c|c|c|c|c|c|c|c|}
\hline 64.83 & - & -- & - & D & - & -- & - & - & 03N04W21ABDB01 \\
\hline 40.88 & 25 & 1.3 & D & $\mathbf{D}$ & $06-20-91$ & 614 & 8.2 & 12.5 & 02N05W27CBCB01 \\
\hline 67.02 & 25 & + & D & $\mathbf{D}$ & $06-19-91$ & 299 & 7.9 & 12.0 & 02N04W04DCCCOI \\
\hline 18.72 & 35 & .7 & D & $\mathbf{D}$ & $06-20-91$ & 701 & 7.3 & 10.5 & 02N04W28CDCB01 \\
\hline 59.32 & - & -- & - & - & -- & - & -- & - & 02N03W33DCD 01 \\
\hline 48.12 & -- & -- & - & - & -- & - & - & -- & 02N03W34CCD 01 \\
\hline 55.04 & 30 & 1.7 & D & $\mathbf{D}$ & $06-20-91$ & 1,210 & 6.9 & 11.5 & 01N05W03CCAC01 \\
\hline 85.92 & 60 & -- & $\mathbf{D}$ & $\mathbf{D}$ & -- & -- & -- & -- & 01N05W22AAAA01 \\
\hline 103.97 & 25 & 2.5 & $\mathbf{D}$ & D & $06-22-91$ & 790 & 7.8 & 12.0 & 01N05W24DCCA01 \\
\hline 10.35 & -- & - & - & - & -- & -- & -- & -- & 01N04W03BADD0I \\
\hline- & -- & -- & - & D & -- & -- & -- & - & 0IN04W04ABBA01 \\
\hline 22.64 & - & - & - & $\mathbf{D}$ & -- & -- & -- & - & 01N04W04ACCDOI \\
\hline-- & 450 & 230 & $\mathbf{D}$ & $\mathbf{D}$ & -- & - & -- & - & 01N04W04ADDA01 \\
\hline 193.60 & 18 & 1.0 & $\boldsymbol{D}$ & D & $06-22-91$ & 322 & 7.7 & 11.5 & 01N04W07BACA01 \\
\hline 14.43 & 20 & -- & $\mathbf{D}$ & D & $06-18-91$ & 471 & 8.5 & 10.0 & OIN03W03DAA 01 \\
\hline 162.88 & 30 & .7 & $\mathrm{D}$ & D & $06-21-91$ & 688 & 7.9 & 13.0 & 0IN03W17ABDA01 \\
\hline 55.47 & 30 & 2.0 & D & $\mathbf{D}$ & $06-20-91$ & 248 & 7.2 & 9.5 & 01S05W14BBBC01 \\
\hline- & - & -- & - & - & $-\cdot$ & -- & - & -- & 01S05W26ADCC01 \\
\hline 7.76 & 500 & 13 & $\mathbf{D}$ & $\mathbf{D}$ & $\cdots$ & - & - & -- & 01S05W26DCD 01 \\
\hline 35.29 & 20 & 1.2 & D & $\mathbf{D}$ & $06-21-91$ & - & 7.5 & 14.0 & 01S04W06DDBC01 \\
\hline
\end{tabular}

09S12W22DCBD01 09S 1 2W22DCBD02 09S12W29CBBB01 09S12W33DAAA01 09S12W34CBAB01

10S14W03BBCB01 1OS I4W10BDAD01 10S14W23CDBB0 1 I0S13W03BBAA01 10S12W11AABD01

$\begin{array}{ccc}7.0 & 8.5 & \text { 10S12W25AACB01 } \\ -- & -- & \text { 10S12W27CADA01 } \\ -- & 9.0 & \text { 10S11W08BCBA01 }\end{array}$
10S11W08BCBA01 
Table 1. Records of selected wells by basin, Northern Rocky Mountains intermontane basins, Montana (Continued)

\begin{tabular}{|c|c|c|c|c|c|c|c|c|c|}
\hline Well number & $\begin{array}{l}\text { Altitude } \\
\text { of land } \\
\text { surface } \\
\text { (ft) }\end{array}$ & $\begin{array}{l}\text { Date well } \\
\text { conetructed }\end{array}$ & $\begin{array}{c}\text { Geologic } \\
\text { unit }\end{array}$ & $\begin{array}{l}\text { Depth } \\
\text { of } \\
\text { well } \\
\text { (fi) }\end{array}$ & $\begin{array}{c}\text { Diam- } \\
\text { oter } \\
\text { of } \\
\text { cesing } \\
\text { (In.) }\end{array}$ & $\begin{array}{c}\text { Type } \\
\text { of } \\
\text { finish }\end{array}$ & $\begin{array}{c}\text { Top of } \\
\text { open } \\
\text { Inter. } \\
\text { val } \\
\text { (ft) }\end{array}$ & $\begin{array}{l}\text { Primary } \\
\text { use of } \\
\text { water }\end{array}$ & $\begin{array}{c}\text { Date water } \\
\text { level } \\
\text { measured }\end{array}$ \\
\hline \multicolumn{10}{|c|}{ Jefferson River Valley-Continued } \\
\hline 02S06W12CCBC01 & 4,590 & $03-25-80$ & TKi & 139 & 6.0 & $\mathbf{P}$ & 100 & $\mathbf{U}$ & $06-21-91$ \\
\hline 02S06W 12CCCC 01 & 4,555 & $11-19-82$ & Ts & 115 & 6.0 & $\mathbf{P}$ & 75 & $\mathbf{H}$ & $06-21-91$ \\
\hline 02S06W35DCB 01 & 4,595 & $01-22-87$ & Qal & 56 & 6.0 & $\mathbf{P}$ & 47 & $\mathbf{H}$ & $06-22-91$ \\
\hline 03S06W11BAAA01 & 4,635 & 07-06-79 & Qal & 60 & 6.0 & $\mathbf{P}$ & 55 & $\mathbf{H}$ & $06-21-91$ \\
\hline 03S05W18DBCA01 & 5,140 & --51 & Ts & 200 & 8.0 & - & -- & $\mathbf{s}$ & -- \\
\hline \multicolumn{10}{|c|}{ Jocko River Valley } \\
\hline 17N20W16CBAA01 & 2,790 & $09-13-83$ & Qal & 18 & 2.0 & $\mathbf{P}$ & 8 & $\mathbf{U}$ & 08-01-91 \\
\hline 17N20W35DABD01 & 2,960 & $11-04-77$ & Qal & 50 & 6.0 & $\mathbf{O}$ & -- & $\mathbf{U}$ & 08-01-91 \\
\hline 16N19W08ACBD01 & 3,300 & 09-28-88 & Qal & 307 & 6.0 & $\mathbf{P}$ & 198 & $\mathbf{U}$ & $07-11-91$ \\
\hline $16 \mathrm{~N} 19 \mathrm{~W} 09 \mathrm{CC} \Lambda \mathrm{B} 02$ & 3,300 & $09-06-69$ & Qal & 154 & 6.0 & $\mathbf{P}$ & 135 & $\mathbf{U}$ & 04-05-89 \\
\hline \multicolumn{10}{|c|}{ Kalispell Valley } \\
\hline 34N24W08BADA01 & 3,240 & -- & Qal & 47 & - & - & -. & $\mathbf{H}$ & $08-07-91$ \\
\hline 34N24W20CCBD01 & 3,760 & $10-05-89$ & QTsu & 84 & 6.0 & $\mathbf{P}$ & 74 & $\mathbf{H}$ & $08-09-91$ \\
\hline $33 \mathrm{~N} 24 \mathrm{~W} 23 \mathrm{ACAB01}$ & 3,220 & $12-08-87$ & QTsu & 78 & 6.0 & 0 & $\ldots$ & $\mathbf{H}$ & $08-08-91$ \\
\hline 33N23W30BDBD01 & 3,250 & $07-27-83$ & Qal & 35 & 6.0 & o & -- & $\mathbf{P}$ & $08-08-91$ \\
\hline 32N34W09CDDA01 & 1,855 & -- & Qal & 28 & -- & - & - & $\mathbf{H}$ & $08-19-91$ \\
\hline 32N23W07BABB01 & 3,175 & 08-03-87 & Qal & 156 & 8.0 & $\mathrm{o}$ & -- & $\mathbf{P}$ & $08-07-91$ \\
\hline 32N23W08CAD 01 & 3,380 & $08-26-86$ & QTsu & 310 & 6.0 & $\mathbf{O}$ & - & $\mathbf{H}$ & $08-07-91$ \\
\hline 32N23W17BBBC01 & 3,158 & $08-28-64$ & QTsu & 50 & 6.0 & 0 & -- & $\mathbf{P}$ & $08-07-91$ \\
\hline 3 IN23W02ADAB01 & 3,205 & $10-08-74$ & Qal & 152 & 6.0 & $\mathbf{P}$ & 147 & $\mathbf{H}$ & $08-06-91$ \\
\hline $31 \mathrm{~N} 23 \mathrm{~W} 11 \mathrm{BBC} \quad 01$ & 3,050 & $07-06-85$ & Qal & 146 & 7.0 & o & -- & $\mathbf{H}$ & 08-09-91 \\
\hline 31N23W25ACCA01 & 3,020 & $10-03-88$ & Qal & 188 & 6.0 & o & -- & $\mathbf{H}$ & $08-10-91$ \\
\hline 31N23W36BDBA01 & 3,020 & $06-07-83$ & Yms & 107 & 6.0 & $\mathbf{P}$ & 104 & $\mathbf{H}$ & $08-10-91$ \\
\hline $31 \mathrm{~N} 21 \mathrm{~W} 29 \mathrm{CBCD} 01$ & 3,055 & 03-08-88 & Qal & 254 & 6.0 & o & -- & $\mathbf{H}$ & $08-20-91$ \\
\hline $31 \mathrm{~N} 21 \mathrm{~W} 34 \mathrm{AADC0} 1$ & 3,150 & $06-11-86$ & Qal & 315 & 6.0 & 0 & -- & $\mathbf{H}$ & $08-20-91$ \\
\hline $31 \mathrm{~N} 21 \mathrm{~W} 35 \mathrm{DBCC} 01$ & 3,115 & $07-25-85$ & Qal & 317 & 6.0 & $\mathbf{P}$ & 307 & $\mathbf{H}$ & $08-15-91$ \\
\hline 31N20W31CCBA01 & 3,280 & $08-18-82$ & Qal & 356 & 6.0 & o & - & $\mathbf{H}$ & $08-20-91$ \\
\hline 30N22W0IDBDD01 & 3,030 & $-\quad-65$ & Qal & 223 & 7.0 & - & - & $\mathbf{H}$ & $08-17-91$ \\
\hline 30N22W08CDDA01 & 3,015 & $06-18-79$ & Qal & 363 & 6.0 & $\mathbf{O}$ & -- & $\mathbf{H}$ & $08-11-91$ \\
\hline 30N22W08DCCD01 & 3,013 & $05-24-79$ & Yms & 630 & 6.0 & 0 & - & H & $08-11-91$ \\
\hline 30N22W 18BBBD01 & 3,050 & $11-20-78$ & Yms & 383 & 8.0 & $\mathbf{P}$ & 323 & $\mathbf{T}$ & $08-10-91$ \\
\hline $30 \mathrm{~N} 22 \mathrm{~W} 25 \mathrm{AACC} 01$ & 3,227 & $07-02-63$ & Qal & 372 & 6.0 & o & -- & $\mathbf{H}$ & 08-09-91 \\
\hline 30N22W25DAB OI & 3,195 & $09-23-66$ & Qal & 284 & 7.0 & $\mathbf{O}$ & - & $\mathbf{H}$ & $08-09-91$ \\
\hline 30N22W26CDAD0I & 3,022 & $12-07-84$ & Yms & 228 & 6.0 & $\mathbf{P}$ & 166 & $\mathbf{H}$ & $08-20-91$ \\
\hline 30N22W34CBDD01 & 2,985 & $04-24-78$ & Qal & 250 & 6.0 & $\mathbf{P}$ & 246 & $\mathbf{H}$ & $08-15-91$ \\
\hline 30N21W05BCBC01 & 3,025 & $09-07-79$ & Qal & 381 & 6.0 & 0 & -- & $\mathbf{P}$ & $08-09-91$ \\
\hline
\end{tabular}


Table 1. Records of selected wells by basin, Northern Rocky Mountains intermontane basins, Montana (Continued)

\begin{tabular}{|c|c|c|c|c|c|c|c|c|c|}
\hline $\begin{array}{c}\text { Water level } \\
\text { (ft) }\end{array}$ & $\begin{array}{c}\text { Dls- } \\
\text { charge } \\
\text { (gal/ } \\
\text { min) }\end{array}$ & $\begin{array}{c}\text { Specific } \\
\text { capacity } \\
\text { [(gal/ } \\
\text { min)/ft] }\end{array}$ & $\begin{array}{l}\text { Source } \\
\text { of dls- } \\
\text { charge } \\
\text { data }\end{array}$ & $\begin{array}{l}\text { Type } \\
\text { of log } \\
\text { avalt } \\
\text { able }\end{array}$ & $\begin{array}{c}\text { Date } \\
\text { water- } \\
\text { quallty } \\
\text { parameler } \\
\text { measured }\end{array}$ & $\begin{array}{c}\text { Specific } \\
\text { conduc- } \\
\text { tance } \\
\text { ( } \mu \mathrm{S} / \mathrm{cm})\end{array}$ & $\begin{array}{l}\text { pH } \\
\text { (atan- } \\
\text { dard } \\
\text { units) }\end{array}$ & $\begin{array}{l}\text { Tem- } \\
\text { per- } \\
\text { ature } \\
\left({ }^{\circ} \mathrm{C}\right)\end{array}$ & Wall number \\
\hline
\end{tabular}

\section{Jefferson River Valley-Continued}

$\begin{array}{cccccccccc}46.21 & 8 & 2.1 & \text { D } & \text { D } & 06-21-91 & 941 & 7.6 & 12.5 & \text { 02S06W12CCBC01 } \\ 26.02 & 8 & -- & \text { D } & \text { D } & 06-21-91 & 401 & 7.2 & 13.5 & \text { 02S06W12CCCC01 } \\ 24.06 & 20 & 5.0 & \text { D } & \text { D } & 06-22-91 & 516 & 7.6 & 12.0 & \text { 02S06W35DCB 01 } \\ 40.57 & 25 & 1.2 & \text { D } & \text { D } & 06-21-91 & 358 & 7.8 & 12.5 & \text { 03S06W11BAAA01 } \\ -- & -- & -- & - & - & 06-22-91 & 285 & 8.4 & 15.0 & 03 S 05 W 18 D B C A 01\end{array}$

\begin{tabular}{|c|c|c|c|c|c|c|c|c|c|}
\hline 5.03 & -- & -- & - & D & -- & - & -- & -- & $17 \mathrm{~N} 20 \mathrm{~W} 16 \mathrm{CBAA01}$ \\
\hline 16.73 & 60 & 20 & $\mathbf{D}$ & $\mathbf{D}$ & -- & -- & -- & -- & 17N20W35DABD01 \\
\hline 142.58 & -- & -- & - & 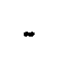 & -- & -- & -- & -- & 16N19W08ACBD \\
\hline 124.90 & 12 & -- & $\mathbf{D}$ & D & -- & -- & -- & .- & $16 \mathrm{~N} 19 \mathrm{~W} 09 \mathrm{CCAB}$ \\
\hline
\end{tabular}

\section{Kalispell Valley}

\begin{tabular}{|c|c|c|c|c|c|c|c|c|c|}
\hline 34.16 & -- & - & - & - & $08-07-91$ & 272 & 7.8 & 7.5 & 34N24W08BADA01 \\
\hline 26.17 & 11 & .3 & $\mathbf{D}$ & $\mathbf{D}$ & -- & -- & - & - & $34 \mathrm{~N} 24 \mathrm{~W} 20 \mathrm{CCBD} 01$ \\
\hline 38.35 & 40 & - & $\mathbf{D}$ & $\mathrm{D}$ & -- & -- & -- & $-\cdot$ & 33N24W23ACAB01 \\
\hline 18.91 & 15 & 1.8 & D & $\mathbf{D}$ & -- & -- & -- & -- & 33N23W30BDBD01 \\
\hline 22.38 & -- & -- & - & - & - & - & - & - & $32 \mathrm{~N} 34 \mathrm{~W} 09 \mathrm{CDDA01}$ \\
\hline 20.00 & 75 & .6 & D & $\mathbf{D}$ & -- & - & -- & -- & 32N23W07BABBO1 \\
\hline 216.63 & 20 & .3 & D & D & -- & -- & - & - & 32N23W08CAD 01 \\
\hline 20.40 & 72 & 5.5 & D & $\mathbf{D}$ & -- & -- & - & - & $32 N 23 W 17 B B B C 01$ \\
\hline 32.74 & 10 & .1 & $\mathbf{D}$ & $\mathbf{D}$ & $08-06-91$ & 793 & 7.4 & 14.5 & $31 N 23 W 02 A D A B 01$ \\
\hline 48.25 & 40 & .4 & $\mathbf{D}$ & $\mathbf{D}$ & $08-09-91$ & 383 & -- & 10.5 & $31 \mathrm{~N} 23 \mathrm{~W} 11 \mathrm{BBC} \quad 01$ \\
\hline 45.92 & 22 & .3 & D & D & $08-10-91$ & 594 & -- & 9.0 & $31 \mathrm{~N} 23 \mathrm{~W} 25 \mathrm{ACCA01}$ \\
\hline 50.59 & 15 & .4 & $\mathbf{D}$ & $\mathbf{D}$ & $08-10-91$ & 555 & -- & 8.5 & $31 \mathrm{~N} 23 \mathrm{~W} 36 \mathrm{BDBA01}$ \\
\hline 11.76 & 25 & .1 & $\mathrm{D}$ & D & $08-20-91$ & 571 & -- & 10.0 & $31 \mathrm{~N} 21 \mathrm{~W} 29 \mathrm{CBCD} 01$ \\
\hline 190.58 & 7 & .1 & $\mathbf{D}$ & $\mathrm{D}$ & $08-20-91$ & 474 & -- & 10.5 & 31 N21W34AADC01 \\
\hline 149.15 & 15 & .2 & $\mathbf{D}$ & $\mathbf{D}$ & -- & -- & -- & - & $31 \mathrm{~N} 21 \mathrm{~W} 35 \mathrm{DBCC} 01$ \\
\hline 274.85 & 17 & .3 & D & $\mathbf{D}$ & $08-20-91$ & 441 & -. & 9.5 & 31 N20W31CCBA01 \\
\hline 77.16 & 20 & .6 & $\mathbf{S}$ & $\mathbf{D}$ & -. & - & -- & - & 30N22W01DBDD01 \\
\hline 27.17 & 20 & .1 & D & $\mathbf{D}$ & $08-11-91$ & 462 & - & 10.0 & $30 \mathrm{~N} 22 \mathrm{W08CDDA01}$ \\
\hline 22.38 & 23 & .2 & $\mathbf{D}$ & $\mathbf{D}$ & $08-11-91$ & 307 & -- & 14.5 & 30N22W08DCCD01 \\
\hline 25.42 & 50 & 2.0 & $\mathbf{D}$ & $\mathbf{D}$ & -- & - & - & -- & $30 \mathrm{~N} 22 \mathrm{~W} 18 \mathrm{BBBD} 01$ \\
\hline 129.02 & 50 & 3.3 & $\mathbf{D}$ & $\mathbf{D}$ & -- & -- & -- & -- & $30 \mathrm{~N} 22 \mathrm{~W} 25 \mathrm{AACCO}$ \\
\hline 259.25 & 30 & 7.5 & $\mathbf{D}$ & D & -- & -- & -- & - & 30N22W25DAB 01 \\
\hline 47.35 & 20 & .3 & $\mathbf{D}$ & $\mathbf{D}$ & $08-20-91$ & 444 & - & 10.0 & $30 \mathrm{~N} 22 \mathrm{~W} 26 \mathrm{CDAD0} 1$ \\
\hline 3.06 & 80 & 20 & $\mathbf{D}$ & D & $08-15-91$ & -- & - & 10.5 & 30N22W34CBDD01 \\
\hline 53.74 & 20 & .1 & D & $\mathbf{D}$ & - & -- & -- & -- & $30 \mathrm{~N} 21 \mathrm{~W} 05 \mathrm{BCBCO} 1$ \\
\hline
\end{tabular}


Table 1. Records of selected wells by basin, Northern Rocky Mountains intermontane basins, Montana (Continued)

\begin{tabular}{|c|c|c|c|c|c|c|c|c|c|}
\hline Well number & $\begin{array}{c}\text { Altitude } \\
\text { of land } \\
\text { surface } \\
\text { (ft) }\end{array}$ & $\begin{array}{c}\text { Date well } \\
\text { constructed }\end{array}$ & $\begin{array}{c}\text { Geologic } \\
\text { unit }\end{array}$ & $\begin{array}{c}\text { Depth } \\
\text { of } \\
\text { well } \\
\text { (ft) }\end{array}$ & $\begin{array}{c}\text { Dlam- } \\
\text { etor } \\
\text { of } \\
\text { caoing } \\
\text { (in.) } \\
\end{array}$ & $\begin{array}{c}\text { Type } \\
\text { of } \\
\text { finieh }\end{array}$ & $\begin{array}{l}\text { Top of } \\
\text { open } \\
\text { Inter- } \\
\text { val } \\
\text { (fi) } \\
\end{array}$ & $\begin{array}{l}\text { Primary } \\
\text { use of } \\
\text { water }\end{array}$ & $\begin{array}{c}\text { Date water } \\
\text { level } \\
\text { measured }\end{array}$ \\
\hline \multicolumn{10}{|c|}{ Kalispell Valley-Continued } \\
\hline 30N21W15BBAA01 & 3,070 & $04-24-85$ & Qal & 350 & 6.0 & $\mathrm{O}$ & -- & $\mathbf{U}$ & $08-15-91$ \\
\hline 30N2IW15BBAD01 & 3,068 & $05-12-86$ & Qal & 154 & 6.0 & $\mathrm{O}$ & -- & $\mathbf{H}$ & $08-15-91$ \\
\hline 30N21W2ICBAD01 & 3,090 & $11-09-86$ & Qal & 236 & 6.0 & $\mathrm{O}$ & -- & HI & $08-21-91$ \\
\hline $30 \mathrm{~N} 21 \mathrm{~W} 22 \mathrm{CDDC0} 1$ & 3,045 & $09-14-87$ & Qal & 295 & 6.0 & $\mathrm{o}$ & .- & $\mathbf{H}$ & $08-19-91$ \\
\hline $30 \mathrm{~N} 21 \mathrm{~W} 23 \mathrm{AAAB} 01$ & 2,985 & $10-22-86$ & Qal & 26 & 6.0 & $\mathbf{P}$ & 16 & $\mathbf{U}$ & $08-20-91$ \\
\hline 30N21W23AAAB02 & 2,985 & $07-17-89$ & Qal & 168 & 6.0 & 0 & - & $\mathbf{H}$ & $08-20-91$ \\
\hline $30 \mathrm{~N} 21 \mathrm{~W} 26 \mathrm{CCDD} 01$ & 2,975 & $09-01-80$ & Qal & 140 & 6.0 & $\mathbf{P}$ & 132 & $\mathbf{H}$ & $08-19-91$ \\
\hline $30 \mathrm{~N} 21 \mathrm{~W} 30 \mathrm{AADCO}$ & 3,245 & $09-30-65$ & Qal & 390 & 6.0 & $\mathbf{P}$ & 355 & $\mathbf{H}$ & $03-24-78$ \\
\hline 30N21W30ADAD01 & 3,270 & $11-21-84$ & Qal & 450 & 8.0 & $\mathbf{O}$ & - & $\mathbf{H}$ & - \\
\hline 30N21W30DAA 01 & 3,260 & $10-25-78$ & Qal & 455 & 10.0 & $\mathbf{P}$ & 455 & $\mathbf{H}$ & $10-25-78$ \\
\hline 30N2OW02CCAA02 & 3,123 & $10-28-54$ & Qal & 162 & 16.0 & $\mathbf{S}$ & 138 & $\mathbf{N}$ & $08-08-91$ \\
\hline 30N20W02CDBA01 & 3,130 & $08-14-54$ & Qal & 175 & 16.0 & $\mathbf{P}$ & 154 & $\mathbf{N}$ & $08-08-91$ \\
\hline 30N20W03DDDA01 & 3,010 & $11-06-56$ & Qal & 70 & 18.0 & $\mathbf{S}$ & 62 & $\mathbf{N}$ & $11-06-56$ \\
\hline 30N20W03DDDA02 & 3,010 & $01-30-57$ & Qal & 62 & 18.0 & $\mathbf{s}$ & 54 & $\mathbf{N}$ & 08-08-91 \\
\hline 30N20W08DAD 01 & 3,010 & $05-11-54$ & Qal & 32 & 16.0 & $\mathbf{S}$ & 17 & $\mathbf{U}$ & $08-08-91$ \\
\hline 30N20W09CBCB01 & 3,010 & $08-\quad-54$ & Qal & 35 & 16.0 & - & -- & $\mathbf{U}$ & 08-08-91 \\
\hline $30 \mathrm{~N} 20 \mathrm{~W} 18 \mathrm{ABAC01}$ & 3,065 & 06-05-79 & Qal & 231 & 12.0 & $\mathbf{P}$ & 196 & $\mathbf{P}$ & -- \\
\hline 30N20W I 8CDCA01 & 3,045 & $12-11-67$ & Qal & 210 & 10.0 & $\mathrm{O}$ & - & $\mathbf{P}$ & $08-08-91$ \\
\hline $30 \mathrm{~N} 20 \mathrm{~W} 18 \mathrm{CDCA02}$ & 3,020 & $07-14-78$ & Qal & 270 & 10.0 & $\mathbf{S}$ & 237 & $\mathbf{P}$ & $07-14-78$ \\
\hline $30 \mathrm{~N} 20 \mathrm{~W} 21 \mathrm{ABABO} 1$ & 3,085 & $10-06-87$ & Qal & 250 & 6.0 & $\mathbf{P}$ & 240 & $\mathrm{~T}$ & $08-14-91$ \\
\hline $30 \mathrm{~N} 20 \mathrm{~W} 22 \mathrm{DDBC} 01$ & 3,075 & $05-19-81$ & Qal & 250 & 6.0 & o & -- & $\mathbf{H}$ & $08-15-91$ \\
\hline 30N20W27CBCD01 & 3,058 & $03-30-66$ & Qal & 334 & 7.0 & $\mathrm{o}$ & -- & $\mathbf{H}$ & $08-17-91$ \\
\hline 30N20W28DACC01 & 3,065 & $05-10-76$ & Qal & 293 & 12.0 & $\mathbf{P}$ & 150 & $\mathbf{I}$ & $08-17-91$ \\
\hline 30N20W29BCBC0I & 3,065 & 08-01-88 & Qal & 152 & 6.0 & $\mathrm{O}$ & -- & $\mathbf{H}$ & $08-17-91$ \\
\hline $30 \mathrm{~N} 20 \mathrm{~W} 30 \mathrm{BAAA01}$ & 2,990 & $03-31-83$ & Qal & 212 & 8.0 & $\mathbf{P}$ & 96 & $\mathbf{P}$ & $08-17-91$ \\
\hline 29N22W02AAAC01 & 3,100 & 03-01-83 & Qal & 213 & 6.0 & $\mathrm{O}$ & - & $\mathbf{H}$ & $08-20-91$ \\
\hline 29N22W08ADDD01 & 3,120 & $01--64$ & Qg & 211 & 7.0 & o & -- & $\mathbf{H}$ & $08-07-91$ \\
\hline 29N22W 14BBDD0 1 & 3,055 & --40 & Qg & 220 & 5.0 & $\mathbf{O}$ & -- & $\mathbf{u}$ & $08-13-91$ \\
\hline 29N22W15BBDD01 & 3,065 & $06-28-74$ & Qal & 470 & 16.0 & $\mathbf{P}$ & 235 & $\mathbf{I}$ & 08-11-91 \\
\hline $29 \mathrm{~N} 22 \mathrm{~W} 26 \mathrm{BCBC} 01$ & 3,060 & $09-08-75$ & Qg & 313 & 8.0 & $\mathbf{P}$ & 290 & $\mathbf{H}$ & $08-13-91$ \\
\hline 29N22W28ACCC01 & 3,090 & $-\quad-02$ & Qg & 200 & 36.0 & o & - & $\mathbf{H}$ & $08-13-91$ \\
\hline 29N22W28DDDD01 & 3,070 & $-\quad-45$ & Qg & 180 & 5.0 & O & - & $\mathbf{S}$ & 08-14-91 \\
\hline 29N22W36BCBD01 & 3,050 & $07-31-71$ & Qg & 452 & 6.6 & $\mathbf{P}$ & 330 & $\mathbf{U}$ & $10-25-91$ \\
\hline 29N21W01AAAC01 & 2,965 & $09-19-78$ & Qal & 83 & 6.0 & $\mathrm{O}$ & -- & H & $08-19-91$ \\
\hline 29N21W04DBDA01 & 2,955 & 04-09-63 & Qal & 17 & 48.0 & o & -- & I & 08-19-91 \\
\hline
\end{tabular}


Table 1. Records of selected wells by basin, Northern Rocky Mountains intermontane basins, Montana (Continued)

\begin{tabular}{|c|c|c|c|c|c|c|c|c|c|}
\hline $\begin{array}{c}\text { Water level } \\
\text { (fi) }\end{array}$ & $\begin{array}{c}\text { Die- } \\
\text { charge } \\
\text { (gail } \\
\text { min) }\end{array}$ & $\begin{array}{c}\text { Speclfic } \\
\text { capacity } \\
\text { [(gal/ } \\
\text { min)/ft] }\end{array}$ & $\begin{array}{l}\text { Source } \\
\text { of dis- } \\
\text { charge } \\
\text { data }\end{array}$ & $\begin{array}{l}\text { Type } \\
\text { of log } \\
\text { avall- } \\
\text { able }\end{array}$ & $\begin{array}{c}\text { Date } \\
\text { water- } \\
\text { quallty } \\
\text { parameter } \\
\text { measured }\end{array}$ & 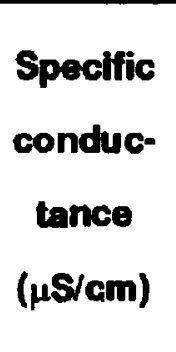 & $\begin{array}{l}\text { pH } \\
\text { (atan- } \\
\text { dard } \\
\text { unlts) }\end{array}$ & $\begin{array}{l}\text { Tem- } \\
\text { per- } \\
\text { ature } \\
\left.\text { ( }{ }^{\circ} \mathrm{C}\right)\end{array}$ & Well number \\
\hline
\end{tabular}

\begin{tabular}{|c|c|c|c|c|c|c|c|c|c|}
\hline 47.59 & 18 & .1 & D & D & -- & -- & -. & -- & 30N21W15BBAA01 \\
\hline 51.34 & 50 & .5 & D & D & $08-15-91$ & 599 & - & 12.0 & 30N21W15BBAD01 \\
\hline 149.09 & 30 & -- & D & D & $08-21-91$ & 421 & -- & 10.0 & 30N21W21CBAD01 \\
\hline 108.63 & 18 & .1 & D & D & $08-19-91$ & 241 & -- & 10.0 & $30 \mathrm{~N} 21 \mathrm{~W} 22 \mathrm{CDDC0} 1$ \\
\hline 12.30 & 35 & 35 & D & $D$ & -- & - & -- & -- & $30 \mathrm{~N} 21 \mathrm{~W} 23 \mathrm{AAAB} 01$ \\
\hline 14.71 & 23 & .2 & D & D & 08-20-91 & 589 & -- & 10.5 & $30 \mathrm{~N} 21 \mathrm{~W} 23 \mathrm{AAAB} 02$ \\
\hline 10.55 & 15 & .6 & D & D & $08-19-91$ & 309 & -- & 10.0 & 30N21W26CCDD01 \\
\hline *324 & 50 & 5.0 & D & D & -- & -- & -- & - & 30N21W30AADC01 \\
\hline-- & 90 & .6 & D & D & -- & - & $\cdots$ & -- & 30N2 I W30ADAD01 \\
\hline *357 & 270 & 4.4 & D & D & -- & - & -- & -- & 30N21W30DAA 01 \\
\hline 112.48 & 900 & 100 & D & D & -- & - & -. & -- & 30N20W02CCAA02 \\
\hline 129.02 & 1,500 & 300 & D & D & -- & -- & -- & -- & 30N20W02CDBA01 \\
\hline 11.60 & 1,400 & 640 & D & D & -- & -- & - & -. & 30N20W03DDDA01 \\
\hline 17.89 & 1,000 & 530 & D & D & -- & -- & -- & -- & 30N20W03DDDA02 \\
\hline 11.72 & 1,400 & -- & $\mathbf{D}$ & D & $\cdots$ & - & -- & -- & 30N20W08DAD 01 \\
\hline 11.67 & 1,400 & -- & $\mathbf{S}$ & - & -- & -- & -- & -- & 30N20W09CBCB01 \\
\hline-- & 1,200 & 13 & D & D & -. & -- & -- & -- & 30N20W I8ABACO1 \\
\hline 71.88 & 60 & 4.3 & D & D & -- & -- & -- & -- & 30N20W18CDCA01 \\
\hline$* 70$ & 300 & 1.9 & D & D & -- & -. & -- & -- & $30 \mathrm{~N} 20 \mathrm{~W} 18 \mathrm{CDCA02}$ \\
\hline 108.57 & 80 & -- & D & D & $08-14-91$ & 355 & - & 10.5 & 30N20W21 ABAB01 \\
\hline 11.96 & 60 & 1.0 & D & D & $08-15-91$ & 563 & -- & 10.5 & $30 \mathrm{~N} 20 \mathrm{~W} 22 \mathrm{DDBC} 01$ \\
\hline 64.41 & -- & -- & - & D & $08-17-91$ & 2,090 & -- & 20.0 & $30 \mathrm{~N} 20 \mathrm{~W} 27 \mathrm{CBCD} 01$ \\
\hline 81.53 & 600 & 3.6 & D & D & $08-17-91$ & 566 & -. & 13.0 & 30N20W28DACC01 \\
\hline 93.08 & 20 & -- & D & D & $08-17-91$ & 411 & -- & 10.0 & $30 \mathrm{~N} 20 \mathrm{~W} 29 \mathrm{BCBC} 01$ \\
\hline 15.90 & 150 & 3.9 & D & D & - & -- & -- & -- & 30N20W30BAAA0I \\
\hline 168.45 & 25 & 1.0 & D & D & $08-20-91$ & 467 & -- & -- & 29N22W02AAAC01 \\
\hline 189.43 & -- & -- & - & - & -- & - & -- & -- & 29N22W08ADDD01 \\
\hline 134.32 & 5 & -- & D & D & -- & - & - & -- & 29N22W14BBDD01 \\
\hline 146.04 & 1,800 & 15 & D & D & -- & -- & -- & - & 29N22W15BBDD01 \\
\hline 149.71 & 50 & 17 & D & D & -- & -- & -- & -- & $29 \mathrm{~N} 22 \mathrm{~W} 26 \mathrm{BCBC} 01$ \\
\hline 160.09 & -- & -- & - & - & -- & - & -- & -- & 29N22W28ACCC01 \\
\hline 166.44 & 10 & -- & D & D & -. & - & -- & -- & 29N22W28DDDD01 \\
\hline 140.18 & 110 & 110 & D & - & -- & -- & -- & -- & 29N22W36BCBD01 \\
\hline 13.44 & 15 & .3 & D & D & $08-19-91$ & 401 & -- & 8.5 & 29N2 I W01 AAAC01 \\
\hline 9.15 & -- & -- & - & D & $08-19-91$ & 416 & -- & -- & 29N2 1 W04DBDA01 \\
\hline
\end{tabular}


Table 1. Records of selected wells by basin, Northern Rocky Mountains intermontane basins, Montana (Continued)

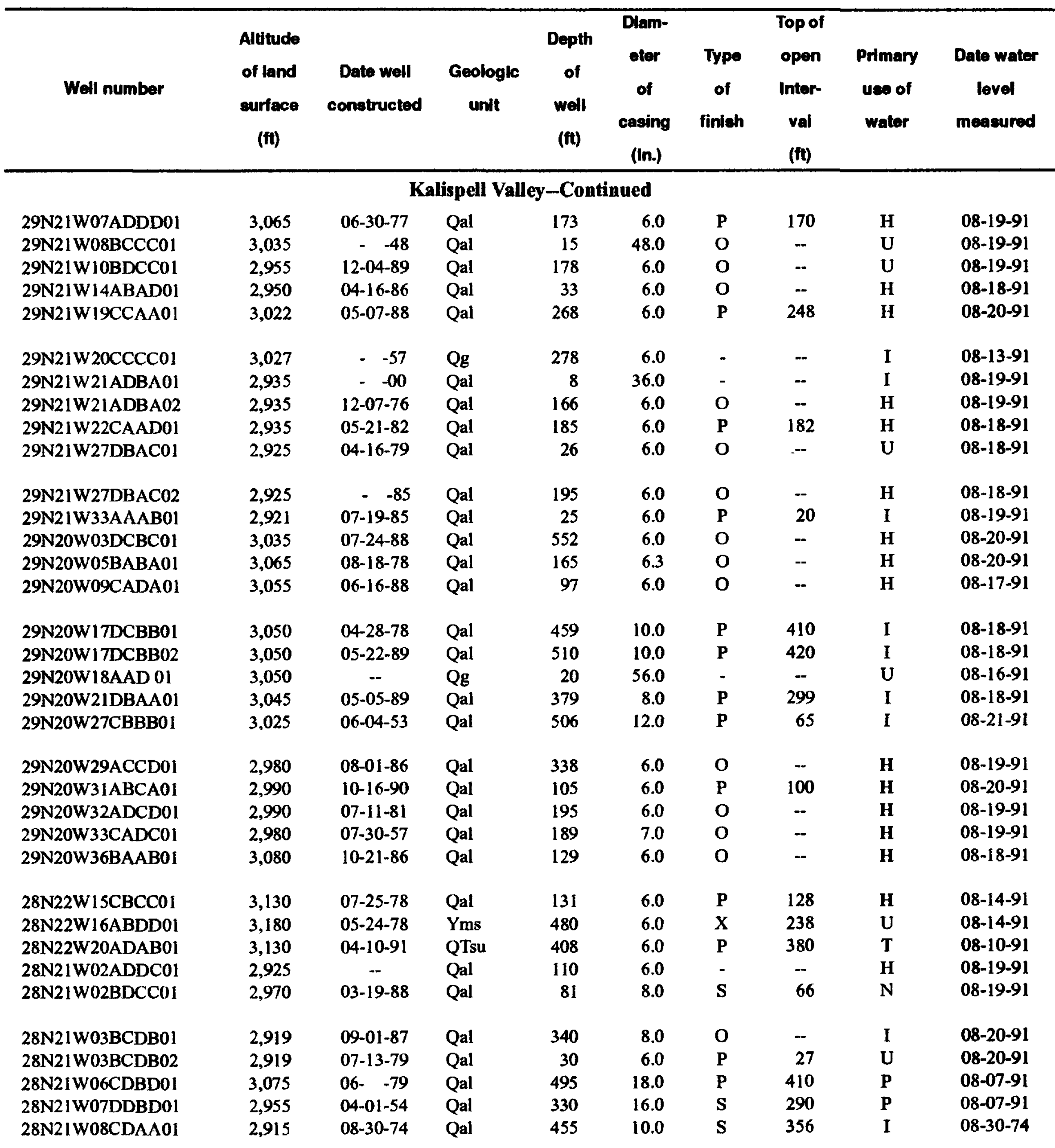


Table 1. Records of selected wells by basin, Northern Rocky Mountains intermontane basins, Montana (Continued)

\begin{tabular}{|c|c|c|c|c|c|c|c|c|c|}
\hline $\begin{array}{c}\text { Water level } \\
\text { (ft) }\end{array}$ & $\begin{array}{c}\text { Dis- } \\
\text { charge } \\
\text { (gal/ } \\
\text { min) }\end{array}$ & $\begin{array}{c}\text { Specific } \\
\text { capacity } \\
\text { [(gal } \\
\text { min)/ft] }\end{array}$ & $\begin{array}{l}\text { Source } \\
\text { of die- } \\
\text { charge } \\
\text { data }\end{array}$ & $\begin{array}{l}\text { Type } \\
\text { of iog } \\
\text { avall- } \\
\text { abla }\end{array}$ & $\begin{array}{c}\text { Dato } \\
\text { water- } \\
\text { quality } \\
\text { parameter } \\
\text { meacured }\end{array}$ & $\begin{array}{c}\text { Specific } \\
\text { conduc- } \\
\text { tance } \\
(\mu \mathrm{S} / \mathrm{cm})\end{array}$ & $\begin{array}{c}\text { pH } \\
\text { (stan- } \\
\text { dard } \\
\text { unlts) }\end{array}$ & $\begin{array}{l}\text { Tem- } \\
\text { per- } \\
\text { ature } \\
\left({ }^{\circ} \mathrm{C}\right)\end{array}$ & Well number \\
\hline
\end{tabular}

\begin{tabular}{|c|c|c|c|c|c|c|c|c|c|}
\hline \\
\hline 123.75 & 20 & $\cdots$ & D & D & $08-19-91$ & 661 & -- & 10.0 & 29N21W07ADDD01 \\
\hline 8.15 & 30 & -- & D & D & -- & -- & -- & -- & 29N21 W08BCCC01 \\
\hline 13.73 & 30 & .3 & D & D & - & -- & -- & -- & 29N21W10BDCC01 \\
\hline 9.60 & 20 & 1.8 & D & D & $08-18-91$ & 232 & - & 9.5 & 29N21W14ABAD01 \\
\hline 100.23 & 100 & .7 & D & D & $08-20-91$ & 579 & - & 16.0 & 29N21W19CCAA01 \\
\hline 109.54 & -- & -- & - & D & -- & - & - & -- & $29 \mathrm{~N} 21 \mathrm{~W} 20 \mathrm{CCCC} 01$ \\
\hline 7.89 & 1,000 & -- & D & D & $08-19-91$ & 474 & -- & 11.0 & 29N21W21ADBA01 \\
\hline 10.60 & 50 & 8.3 & D & D & $08-19-91$ & 342 & -- & 10.0 & 29N21W21ADBA02 \\
\hline 5.96 & 50 & .9 & D & D & $08-18-91$ & 326 & -- & 12.0 & 29N21W22CAAD01 \\
\hline 10.45 & 15 & 1.9 & D & $\mathbf{D}$ & -- & -- & -- & -- & 29N21W27DBAC01 \\
\hline .30 & 25 & 13 & D & D & $08-18-91$ & 277 & -- & 13.0 & 29N21W27DBAC02 \\
\hline 11.01 & 40 & 8.0 & D & D & $07-19-85$ & 471 & -- & 10.0 & 29N21W33AAAB01 \\
\hline 33.97 & 30 & .1 & D & D & $08-20-91$ & 867 &.- & 12.5 & 29N20W03DCBC01 \\
\hline 89.48 & 12 & .2 & D & D & $08-20-91$ & 468 & -- & 9.5 & 29N20W05BABA01 \\
\hline 14.41 & 25 & .4 & D & D & $08-17-91$ & 455 & -- & 9.5 & 29N20W09CADA01 \\
\hline *85 & 1,800 & 14 & D & $\mathbf{D}$ & $08-18-91$ & 430 & -- & 11.5 & 29N20W17DCBB01 \\
\hline$* 85$ & 1,300 & -- & D & D & -. & -- & -- & -- & 29N20W17DCBB02 \\
\hline 14.27 & -- & -- & - & - & - & - & -- & -- & 29N20W18AAD 01 \\
\hline$* 80$ & -- & -- & - & D & $08-18-91$ & 422 & -- & 10.5 & 29N20W21DBAA01 \\
\hline 47.35 & 1,500 & -- & D & D & 08-20-91 & 368 & -- & 9.0 & 29N20W27CBBB01 \\
\hline 39.16 & 75 & .3 & D & D & -- & -- & -- & -- & 29N20W29ACCD01 \\
\hline 57.14 & 30 & .8 & $\mathbf{R}$ & D & $08-20-91$ & 332 & -. & 13.5 & 29N20W31ABCA01 \\
\hline 43.10 & 60 & -. & D & D & $08-19-91$ & 420 & -- & 9.0 & 29N20W32ADCD01 \\
\hline 28.46 & 35 & .8 & D & D & 08-19-91 & 484 & -- & 9.0 & 29N20W33CADC01 \\
\hline 103.45 & 16 & -- & D & D & 08-18-91 & 198 & -- & 12.0 & 29N20W36BAAB01 \\
\hline 71.83 & 30 & .5 & D & - & $08-14-91$ & 578 & -- & 10.5 & $28 \mathrm{~N} 22 \mathrm{~W} 15 \mathrm{CBCC} 01$ \\
\hline 73.02 & 10 & .1 & D & D & -- & -- & -- & -- & 28N22W16ABDD01 \\
\hline 43.20 & 3 & -- & D & D & -- & -- & -- & -- & 28N22W20ADAB01 \\
\hline 5.05 & -- & -- & - & - & 08-19-91 & 271 & -- & 10.5 & 28N21 W02ADDC01 \\
\hline 20.47 & 500 & 11 & D & D & $08-19-91$ & 219 & - & 8.0 & 28N21 W02BDCC01 \\
\hline 7.83 & 60 & .5 & D & D & 08-20-91 & 328 & -- & 11.0 & 28N21 W03BCDB01 \\
\hline 9.53 & 35 & 2.3 & D & D & -- & -- & -- & -- & 28N2 1 W03BCDB02 \\
\hline 164.62 & - & -- & - & D & -- & -- & -. & -- & 28N21 W06CDBD01 \\
\hline 51.60 & 1,200 & 18 & $\mathbf{s}$ & D & -- & -- & -- & -- & 28N21W07DDBD01 \\
\hline 13.70 & 2,000 & 72 & $\mathbf{R}$ & D & -- & -- & -- & -- & 28N21 W08CDAA01 \\
\hline
\end{tabular}


Table 1. Records of selected wells by basin, Northern Rocky Mountains intermontane basins, Montana (Continued)

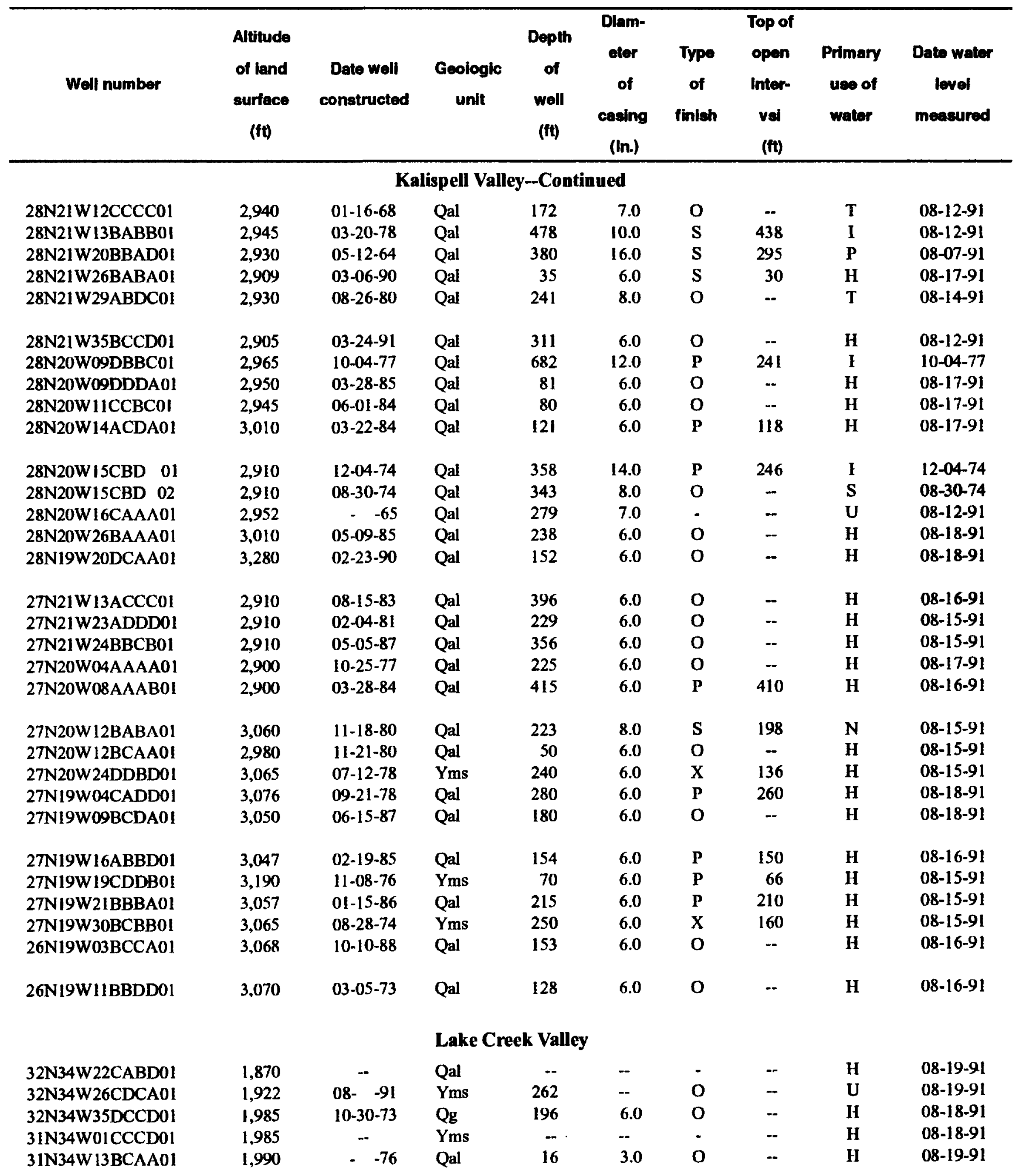


Table 1. Records of selected wells by basin, Northern Rocky Mountains intermontane basins, Montana (Continued)

\begin{tabular}{|c|c|c|c|c|c|c|c|c|c|}
\hline $\begin{array}{c}\text { Water levsl } \\
\text { (fi) }\end{array}$ & $\begin{array}{c}\text { Dis- } \\
\text { charge } \\
\text { (gal/ } \\
\text { min) }\end{array}$ & $\begin{array}{c}\text { Spscific } \\
\text { capacity } \\
\text { [(gsi/ } \\
\text { min)/ft] }\end{array}$ & $\begin{array}{l}\text { Sourcs } \\
\text { of dis- } \\
\text { charge } \\
\text { data }\end{array}$ & $\begin{array}{l}\text { Type } \\
\text { of log } \\
\text { avail- } \\
\text { able }\end{array}$ & $\begin{array}{c}\text { Date } \\
\text { watsr- } \\
\text { quality } \\
\text { parameter } \\
\text { msasured }\end{array}$ & $\begin{array}{l}\text { Specific } \\
\text { conduc- } \\
\text { tance } \\
(\mu S / \mathrm{cm})\end{array}$ & $\begin{array}{c}\text { pH } \\
\text { (stan- } \\
\text { dard } \\
\text { units) }\end{array}$ & $\begin{array}{l}\text { Tem- } \\
\text { par- } \\
\text { ature } \\
\left({ }^{\circ} \mathrm{C}\right)\end{array}$ & Woll number \\
\hline
\end{tabular}

Kalispell Valley-Continued

\begin{tabular}{|c|c|c|c|c|c|c|c|c|c|}
\hline 33.75 & 15 & .3 & D & D & $08-12-91$ & 298 & -- & 11.5 & $28 \mathrm{~N} 21 \mathrm{~W} 12 \mathrm{CCCCO}$ \\
\hline 36.10 & 750 & 5.3 & D & D & -- & -- & -- & -- & 28N21W13BABB01 \\
\hline 22.92 & 1,600 & 34 & D & D & -- & -- & -- & -- & 28N21 W20BBAD01 \\
\hline 16.60 & 30 & -- & D & D & $08-17-91$ & 690 & -- & 8.5 & 28N21W26BABA01 \\
\hline 36.28 & 200 & 1.2 & D & D & $08-14-91$ & 485 & -- & 10.5 & $28 \mathrm{~N} 21 \mathrm{~W} 29 \mathrm{ABDC} 01$ \\
\hline 5.73 & 40 & -- & D & D & -- & -- & -- & .. & $28 \mathrm{~N} 21 \mathrm{~W} 35 \mathrm{BCCD} 01$ \\
\hline$* 20$ & 730 & 24 & D & D & -- & -- & -- & -- & 28N20W09DBBC01 \\
\hline 4.73 & 22 & .5 & D & D & $08-17-91$ & 380 & - & 9.0 & 28N20W09DDDA01 \\
\hline 11.10 & 60 & 1.1 & D & D & -- & -- & - & -- & $28 \mathrm{~N} 20 \mathrm{~W} 11 \mathrm{CCBC} 01$ \\
\hline 83.20 & 20 & -- & D & $\mathbf{D}$ & $08-17-91$ & 385 & -- & 9.0 & 28N20W 14ACDA01 \\
\hline$*+30$ & 3,100 & 60 & $\mathrm{D}$ & D & -- & -- & -- & -- & 28N20W15CBD 01 \\
\hline$*+30$ & 200 & 1.5 & D & D & $08-12-91$ & 274 & -- & 8.5 & $28 \mathrm{~N} 20 \mathrm{~W} 15 \mathrm{CBD} \quad 02$ \\
\hline 5.60 & 20 & 20 & $\mathbf{S}$ & - & -- & -- & -- & -- & 28N20W 16CAAA01 \\
\hline 51.36 & 50 & .8 & D & $\mathbf{D}$ & $08-18-91$ & 353 & -. & 9.0 & 28N20W26BAAA01 \\
\hline 97.99 & 25 & .8 & D & D & $08-18-91$ & 367 & -- & 11.0 & 28N19W20DCAA01 \\
\hline 14.65 & 25 & .1 & D & D & $08-16-91$ & 354 & -- & 17.0 & $27 \mathrm{~N} 21 \mathrm{~W} 13 \mathrm{ACCC} 01$ \\
\hline 13.92 & 15 & .1 & D & D & -- & -- & -- & -- & 27N21W23ADDD01 \\
\hline 13.98 & 85 & .3 & D & D & -- & -- & - & - & 27N21 W24BBCB01 \\
\hline+.52 & 40 & 4.0 & D & D & $08-17-91$ & 493 & -- & 10.0 & 27N20W04AAAA01 \\
\hline 4.36 & 40 & 2.2 & D & D & $08-16-91$ & 912 & - & 14.0 & 27N20W08AAAB01 \\
\hline 123.15 & 300 & 4.3 & D & D & -- & -- & -- & - & 27N20W12BABA01 \\
\hline 24.96 & 30 & -. & D & D & -- & -- & -- & -- & 27N20W12BCAA01 \\
\hline 74.94 & 30 & .3 & D & D & -. & -- & -- & -- & $27 \mathrm{~N} 20 \mathrm{~W} 24 \mathrm{DDBD} 01$ \\
\hline 60.42 & 20 & .1 & D & D & $08-18-91$ & 351 & -- & 10.0 & 27N19W04CADD01 \\
\hline 45.75 & 50 & .5 & D & D & $08-18-91$ & 522 & -- & 8.5 & 27N19W09BCDA01 \\
\hline 13.70 & 6 & .3 & D & D & $08-16-91$ & 407 & -- & 9.5 & 27N19W16ABBD01 \\
\hline 21.20 & 25 & 1.3 & D & D & -- & - & -- & - & 27N19W 19CDDB01 \\
\hline 48.58 & 9 & .1 & D & D & $08-15-91$ & 348 & - & 8.5 & 27N19W21BBBA01 \\
\hline 132.63 & 20 & .2 & D & D & -- & - & -- & - & 27N19W30BCBB01 \\
\hline 23.95 & 50 & .4 & D & D & $08-16-91$ & 405 & -- & 9.0 & 26N19W03BCCA01 \\
\hline 34.75 & 20 & 6.7 & D & D & $08-16-91$ & 278 & - & 9.0 & 26N19W11BBDD01 \\
\hline
\end{tabular}

\section{Lake Creek Valley}

$\begin{array}{rccccccccr}31.52 & -- & -- & - & - & - & - & -- & - & \text { 32N34W22CABD01 } \\ 1.47 & -- & -- & - & - & -- & - & - & - & \text { 32N34W26CDCA01 } \\ 125.91 & 9 & .4 & D & D & -- & -- & -- & - & \text { 32N34W35DCCD01 } \\ 79.56 & -- & -- & - & - & - & - & -- & -- & \text { 31N34W01CCCD01 } \\ 1.05 & -- & -- & - & - & -- & -- & - & -- & \text { 31N34W13BCAA01 }\end{array}$


Table 1. Records of selected wells by basin, Northern Rocky Mountains intermontane basins, Montana (Continued)

\begin{tabular}{|c|c|c|c|c|c|c|c|c|c|}
\hline Well number & $\begin{array}{l}\text { Altitude } \\
\text { of land } \\
\text { surface } \\
\text { (ft) }\end{array}$ & $\begin{array}{l}\text { Dato weil } \\
\text { constructed }\end{array}$ & $\begin{array}{c}\text { Geologic } \\
\text { unit }\end{array}$ & $\begin{array}{c}\text { Depth } \\
\text { of } \\
\text { well } \\
\text { (ft) }\end{array}$ & $\begin{array}{c}\text { Dlam- } \\
\text { eter } \\
\text { of } \\
\text { caoing } \\
\text { (In.) }\end{array}$ & $\begin{array}{l}\text { Type } \\
\text { of } \\
\text { finish }\end{array}$ & $\begin{array}{l}\text { Top of } \\
\text { open } \\
\text { Inter- } \\
\text { ved } \\
\text { (ft) }\end{array}$ & $\begin{array}{c}\text { Primary } \\
\text { use of } \\
\text { water }\end{array}$ & $\begin{array}{c}\text { Date water } \\
\text { level } \\
\text { meacured }\end{array}$ \\
\hline \multicolumn{10}{|c|}{ Lake Creek Valley-Continned } \\
\hline 31N33W 18CCAA01 & 1,900 & $08-11-77$ & Qg & 46 & 6.0 & o & -. & $\mathbf{H}$ & 08-15-91 \\
\hline $31 \mathrm{~N} 33 \mathrm{~W} 19 \mathrm{ADBD} 01$ & 2,110 & $08-17-79$ & $\mathbf{Q g}$ & 170 & 6.0 & o & -- & $\mathbf{P}$ & $08-16-91$ \\
\hline 31N33W28DBDA01 & 2,310 & $05-16-79$ & Qg & 52 & 6.0 & o & -- & $\mathbf{H}$ & $08-19-91$ \\
\hline 31N33W29DABA01 & 2,275 & $07-27-79$ & $\mathbf{Q g}$ & 38 & 6.0 & $\mathbf{P}$ & 33 & $\mathbf{H}$ & 08-14-91 \\
\hline $31 \mathrm{~N} 33 \mathrm{~W} 29 \mathrm{DCCC} 02$ & 2,258 & $07-17-80$ & Qg & 45 & 2.0 & $\mathbf{P}$ & 35 & $\mathbf{U}$ & $08-14-91$ \\
\hline 31N33W34BBBD01 & 2,305 & $07-27-78$ & Qg & 46 & 6.0 & $\mathbf{P}$ & - & $\mathbf{H}$ & $08-14-91$ \\
\hline $30 \mathrm{~N} 33 \mathrm{~W} 04 \mathrm{ABBC} 01$ & 2,385 & 08- -90 & $\mathbf{Q g}$ & 276 & -- & - & - & $\mathbf{H}$ & $08-18-91$ \\
\hline 30N33W05AADD01 & 2,140 & $11-13-73$ & Qg & 151 & 6.0 & $\mathbf{P}$ & 147 & $\mathbf{H}$ & $11-13-73$ \\
\hline 30N33W05ABAB01 & 2,114 & $07-16-80$ & Qg & 187 & 2.0 & $\mathbf{P}$ & 177 & $\mathbf{U}$ & $10-16-91$ \\
\hline 30N33W07ADAD01 & 2,150 & --70 & Qal & 14 & 36.0 & - & - & $\mathbf{H}$ & $08-15-91$ \\
\hline 30N33W08AACA01 & 2,340 & --76 & Qg & 12 & 4.0 & o & -- & $\mathbf{H}$ & 08-15-91 \\
\hline 30N33W 18ACDD01 & 2,260 & $07-26-79$ & Qg & 180 & 6.0 & $\mathbf{P}$ & 175 & $\mathbf{H}$ & $08-16-91$ \\
\hline 30N33W19ACCC01 & 2,322 & $-\quad-76$ & Qg & 192 & - & - & - & $\mathbf{H}$ & 08-18-91 \\
\hline 30N33W20AADB01 & 2,370 & 07-19-79 & Qg & 280 & 6.0 & $\mathbf{P}$ & 275 & $\mathbf{H}$ & $08-16-91$ \\
\hline 30N33W20BBBCOI & 2,275 & $08-10-74$ & Qg & 160 & 6.0 & $\mathbf{O}$ & - & $\mathbf{H}$ & $08-18-91$ \\
\hline $30 \mathrm{~N} 33 \mathrm{~W} 29 \mathrm{ACDC} 01$ & 2,445 & -.75 & Qg & 176 & -- & - & -. & $\mathbf{H}$ & $08-17-91$ \\
\hline 30N33W29DCAC01 & 2,448 & $-\quad-89$ & Unk & 135 & -- & - & - & $\mathbf{H}$ & 08-17-91 \\
\hline 30N33W30DAAD01 & 2,258 & $07-09-80$ & Qg & 43 & 2.0 & $\mathbf{P}$ & 38 & $\mathbf{U}$ & 08-16-91 \\
\hline 30N33W30DAAD02 & 2,257 & $07-10-80$ & $\mathrm{Qg}$ & 23 & 2.0 & $\mathbf{P}$ & 18 & $\mathbf{U}$ & $08-16-91$ \\
\hline 30N33W32ACBD01 & 2,480 & --74 & $\mathrm{Qg}$ & 46 & -- & - & - & $\mathbf{H}$ & $08-18-91$ \\
\hline 29N33W08BDAC01 & 2,442 & $07-16-78$ & Qg & 172 & 6.0 & $\mathbf{P}$ & 167 & $\mathbf{H}$ & 08-18-91 \\
\hline 29N33W17ACAA01 & 2,438 & 05-01-80 & Qg & 158 & 6.0 & $\mathbf{P}$ & 153 & $\mathbf{H}$ & $08-17-91$ \\
\hline $29 \mathrm{~N} 33 \mathrm{~W} 20 \mathrm{CCDCO}$ & 2,332 & -- & Qg & 77 & 6.0 & $\mathbf{s}$ & 70 & $\mathbf{U}$ & $08-19-91$ \\
\hline 29N33W32DACD01 & 2,340 & $08-30-78$ & Qg & 115 & 6.0 & $\mathbf{0}$ & -- & H & 08-19-91 \\
\hline 28N33W03BABC0I & 2,345 & $09-14-75$ & $\mathbf{Q g}$ & 78 & 6.0 & o & -- & $\mathbf{H}$ & $08-19-91$ \\
\hline \multicolumn{10}{|c|}{ Libby Creek Valley } \\
\hline 31N32W24DAAC01 & 2,060 & $01-25-83$ & Qal & 46 & 6.0 & $\mathbf{P}$ & 43 & $\mathbf{H}$ & $08-17-91$ \\
\hline 31N31W02AABA01 & 2,645 & $09-02-85$ & Qg & 82 & 6.0 & $\mathbf{P}$ & 76 & $\mathbf{H}$ & $08-19-91$ \\
\hline 31N3IW02BDDA0I & 2,610 & $09-10-87$ & Qg & 44 & 6.0 & $\mathrm{O}$ & -- & H & $08-20-91$ \\
\hline 31 N31W08AAB 01 & 2,519 & $05-09-84$ & Qg & 74 & 6.0 & 0 & -- & $\mathbf{H}$ & $08-19-91$ \\
\hline 31N31W08ADCD01 & 2,510 & $04-20-79$ & Qg & 145 & 6.0 & $\mathbf{S}$ & 140 & H & $08-19-91$ \\
\hline $31 \mathrm{~N} 31 \mathrm{~W}$ 10CBDA01 & 2,545 & $04-13-90$ & Qg & 178 & 6.0 & $\mathbf{P}$ & 152 & $\mathbf{H}$ & $08-20-91$ \\
\hline $31 \mathrm{~N} 31 \mathrm{~W} 19 \mathrm{BCCC} 01$ & 2,160 & $10-22-87$ & Qal & 121 & 6.0 & $\mathbf{o}$ & - & $\mathbf{H}$ & $08-17-91$ \\
\hline $31 \mathrm{~N} 31 \mathrm{~W} 20 \mathrm{CDDD} 01$ & 2,084 & $07-20-64$ & Qal & 54 & 6.0 & $\mathbf{P}$ & 38 & HI & $08-16-91$ \\
\hline $31 \mathrm{~N} 31 \mathrm{~W} 26 \mathrm{CBA} \quad 03$ & 2,317 & $05-03-71$ & Qal & 275 & 6.0 & - & -- & H & $08-17-91$ \\
\hline $31 \mathrm{~N} 31 \mathrm{~W} 26 \mathrm{CBB} \quad 03$ & 2,315 & $06-18-71$ & Qal & 65 & 4.0 & $\mathbf{P}$ & 59 & $\mathbf{H}$ & $08-17-91$ \\
\hline
\end{tabular}


Table 1. Records of selected wells by basin, Northern Rocky Mountains intermontane basins, Montana (Continued)

\begin{tabular}{|c|c|c|c|c|c|c|c|c|c|}
\hline $\begin{array}{c}\text { Water lovel } \\
\text { (fi) }\end{array}$ & $\begin{array}{c}\text { Dia- } \\
\text { charge } \\
\text { (gal/ } \\
\text { m/n) }\end{array}$ & $\begin{array}{c}\text { Specific } \\
\text { capacity } \\
\text { [(gal/ } \\
\text { min)/ft] }\end{array}$ & $\begin{array}{l}\text { Source } \\
\text { of dls- } \\
\text { charge } \\
\text { data }\end{array}$ & $\begin{array}{l}\text { Type } \\
\text { of log } \\
\text { avall- } \\
\text { abla }\end{array}$ & $\begin{array}{c}\text { Data } \\
\text { water- } \\
\text { quallty } \\
\text { paramoter } \\
\text { meesurad }\end{array}$ & $\begin{array}{c}\text { Speclfic } \\
\text { conduc- } \\
\text { tance } \\
(\mu \mathrm{S} / \mathrm{cm})\end{array}$ & $\begin{array}{l}\text { pH } \\
\text { (atan- } \\
\text { dard } \\
\text { units) }\end{array}$ & $\begin{array}{l}\text { Tem- } \\
\text { per- } \\
\text { ature } \\
\left({ }^{\circ} \mathrm{C}\right)\end{array}$ & Wall number \\
\hline \multicolumn{10}{|c|}{ Lake Creek Valley-Continued } \\
\hline 14.85 & 18 & -. & D & D & $09-08-80$ & 122 & 8.4 & 8.5 & $31 \mathrm{~N} 33 \mathrm{~W} 18 \mathrm{CCAA01}$ \\
\hline 146.29 & 40 & 5.7 & D & D & -- & -- & -- & -- & 31 N33W 19ADBDO 1 \\
\hline 41.87 & 12 & -- & D & D & $06-17-80$ & 388 & -- & 11.0 & 31N33W28DBDA01 \\
\hline 25.93 & 10 & -- & $\mathbf{o}$ & D & $09-08-80$ & 455 & 7.5 & 8.5 & 31N33W29DABA01 \\
\hline 33.00 & -- & -- & - & $\mathrm{D}$ & -- & -- & $\cdots$ & $\cdots$ & $31 \mathrm{~N} 33 \mathrm{~W} 29 \mathrm{DCCC} 02$ \\
\hline 21.06 & 20 & -- & D & D & 08-14-91 & 161 & -- & 9.0 & 31 N33W34BBBD01 \\
\hline 242.57 & -- & -- & - & - & -. & -- & -- & - & 30N33W04ABBC01 \\
\hline 1.00 & 20 & 1.8 & D & D & 09-08-80 & 403 & 8.1 & 10.0 & 30N33W05AADD01 \\
\hline+19.54 & $<1$ & - & $\mathbf{S}$ & $\mathrm{D}$ & $06-09-81$ & 380 & 8.0 & 9.5 & 30N33W05ABAB01 \\
\hline 4.75 &.- & -- & - & - & $08-15-91$ & 78 & -- & 15.0 & 30N33W07ADAD01 \\
\hline 6.82 & -- & -- & - & - & -- & -- & - & -- & 30N33W08AACA01 \\
\hline 77.90 & 50 & -- & D & D & - & -- & - & -- & 30N33W 18ACDD0 1 \\
\hline 103.84 & -- & -- & - & - & -- & - & -- & -- & 30N33W 19ACCCOI \\
\hline 132.63 & 15 & -- & D & D & $06-16-80$ & 330 & -- & 9.0 & 30N33W20AADB0 1 \\
\hline 77.39 & 12 & 3.0 & D & $\mathrm{D}$ & 09-06-80 & 150 & 8.5 & 7.0 & $30 \mathrm{~N} 33 \mathrm{~W} 20 \mathrm{BBBC} 01$ \\
\hline 152.83 & -- & -- & - & - & -- & - & - & -- & 30N33W29ACDC01 \\
\hline 65.91 & -- & -- & - & - & -- & -- & -- & -- & 30N33W29DCAC01 \\
\hline 9.69 & -- & -- & - & D & $06-10-81$ & 100 & 8.5 & 7.0 & 30N33W30DAAD01 \\
\hline 8.59 & -- & -- & - & - & $06-10-81$ & 110 & 8.4 & 7.5 & 30N33W30DAAD02 \\
\hline 32.64 & -- & -- & - & - & -- & -- & -- & -- & 30N33W32ACBD01 \\
\hline 128.64 & 10 & .4 & D & D & $08-18-91$ & 92 & -. & 7.0 & 29N33W08BDAC01 \\
\hline 72.43 & 12 & -- & O & $\mathbf{D}$ & $06-12-80$ & 119 & - & 8.0 & 29N33W 17ACAA01 \\
\hline 12.50 & -- & -- & - & - & -- & -- & -- & -- & 29N33W20CCDCD1 \\
\hline 8.71 & 20 & 13 & D & D & $08-19-91$ & 137 & - & 6.0 & 29N33W32DACD01 \\
\hline 17.88 & 30 & 30 & D & D & $06-16-80$ & 173 & -- & 8.0 & 28N33W03BABC01 \\
\hline \multicolumn{10}{|c|}{ Libby Creek Valley } \\
\hline 30.71 & 6 & 3.4 & $\mathbf{S}$ & $\mathrm{D}$ & $08-17-91$ & 169 & 7.3 & 8.0 & 31N32W24DAAC01 \\
\hline 52.22 & 30 & 1.4 & D & D & $08-19-91$ & 443 & 7.0 & 8.0 & 31N31W02AABA01 \\
\hline 19.45 & 10 & 2.5 & D & $\mathrm{D}$ & $08-20-91$ & 188 & 7.2 & 6.5 & 31N31W02BDDA01 \\
\hline 26.96 & 45 & 2.3 & D & $\mathrm{D}$ & 08-19-91 & 289 & 7.2 & 8.0 & 31N31W08AAB 01 \\
\hline 36.06 & 12 & .1 & D & $\mathbf{D}$ & $08-19-91$ & 271 & 7.1 & 8.5 & 31N31W08ADCD01 \\
\hline 136.68 & 6 & -- & D & D & $08-20-91$ & 467 & 7.2 & 8.0 & 31 N31 W10CBDA01 \\
\hline 111.40 & 11 & 11 & $\mathbf{S}$ & D & $08-17-91$ & 137 & 7.1 & 7.5 & 31N31W19BCCC01 \\
\hline 34.24 & 10 & 3.6 & $\mathbf{S}$ & D & $08-16-91$ & 201 & 7.0 & 6.5 & $31 \mathrm{~N} 31 \mathrm{~W} 20 \mathrm{CDDD} 01$ \\
\hline 250.22 & 2 & .2 & D & D & $08-16-91$ & 482 & 7.3 & 13.5 & $31 N 31$ W26CBA 03 \\
\hline 59.92 & 10 & 1.2 & D & D & $08-17-91$ & 533 & 7.0 & 9.5 & $31 \mathrm{~N} 31 \mathrm{~W} 26 \mathrm{CBB} \quad 03$ \\
\hline
\end{tabular}


Table 1. Records of selected wells by basin, Northern Rocky Mountains intermontane basins, Montana (Continued)

\begin{tabular}{|c|c|c|c|c|c|c|c|c|c|}
\hline Well number & $\begin{array}{c}\text { Altitude } \\
\text { of land } \\
\text { surface } \\
\text { (ft) }\end{array}$ & $\begin{array}{l}\text { Date well } \\
\text { constructed }\end{array}$ & $\begin{array}{c}\text { Geologic } \\
\text { unil }\end{array}$ & $\begin{array}{c}\text { Depth } \\
\text { of } \\
\text { well } \\
\text { (ft) }\end{array}$ & $\begin{array}{c}\text { Dlam- } \\
\text { eter } \\
\text { of } \\
\text { casing } \\
\text { (In.) }\end{array}$ & $\begin{array}{l}\text { Type } \\
\text { of } \\
\text { finish }\end{array}$ & $\begin{array}{l}\text { Top of } \\
\text { open } \\
\text { Inter- } \\
\text { val } \\
\text { (ft) }\end{array}$ & $\begin{array}{c}\text { Primary } \\
\text { use of } \\
\text { water }\end{array}$ & $\begin{array}{l}\text { Date water } \\
\text { Iovel } \\
\text { measured }\end{array}$ \\
\hline \multicolumn{10}{|c|}{ Libby Creek Valley-Continued } \\
\hline $31 \mathrm{~N} 31 \mathrm{~W} 33 \mathrm{CCBB} 01$ & 2,060 & $-\quad-72$ & Qal & 40 & 6.0 & - & .. & $\mathbf{H}$ & 04-07-89 \\
\hline $31 \mathrm{~N} 31 \mathrm{~W} 34 \mathrm{CCACO} 1$ & 2,172 & $08-12-75$ & Qal & 145 & 6.0 & $\mathbf{P}$ & 141 & H & $08-19-91$ \\
\hline $31 \mathrm{~N} 31 \mathrm{~W} 35 \mathrm{AAAA} 01$ & 2,265 & $07-22-91$ & $\mathrm{Qg}$ & 244 & 6.0 & $\mathbf{P}$ & 234 & H & $08-18-91$ \\
\hline $31 \mathrm{~N} 31 \mathrm{~W} 35 \mathrm{CAD} 01$ & 2,090 & $05-18-78$ & Qal & 60 & 6.0 & $\mathbf{P}$ & 54 & $\mathbf{H}$ & $08-19-91$ \\
\hline 31N31W35DDBA01 & 2,065 & -- & Qal & 40 & -- & - & -- & $\mathbf{H}$ & 08-18-91 \\
\hline 30N31W03CACD01 & 2,081 & $07-03-85$ & Qal & 22 & 2.0 & G & 19 & $\mathrm{U}$ & 08-14-91 \\
\hline 30N31 W03CACD02 & 2,081 & $07-03-85$ & Qal & 140 & 2.0 & $\mathrm{G}$ & 137 & $\mathbf{U}$ & $08-14-91$ \\
\hline $30 \mathrm{~N} 31 \mathrm{~W} 03 \mathrm{CACD} 03$ & 2,081 & $07-03-85$ & Qal & 164 & 2.0 & G & 161 & $\mathbf{U}$ & $08-14-91$ \\
\hline 30N31W08AAAB01 & 2,445 & $04-14-87$ & Qg & 615 & 6.0 & $\mathrm{O}$ & -- & $\mathbf{H}$ & $04-14-87$ \\
\hline 30N31W10ACAD01 & 2,099 & $09-06-85$ & Qal & 358 & 2.0 & G & 355 & $\mathbf{U}$ & $08-14-91$ \\
\hline 30N31W10ACAD02 & 2,099 & $09-06-85$ & Qal & 400 & 2.0 & $\mathrm{G}$ & 397 & $\mathbf{U}$ & $08-14-91$ \\
\hline 30N31W10ACBB01 & 2,097 & $02-05-85$ & Qal & 34 & 2.0 & G & 31 & $\mathbf{U}$ & $08-14-91$ \\
\hline 30N31W 10ACBB02 & 2,097 & $02-05-85$ & Qal & 52 & 2.0 & G & 49 & $\mathbf{U}$ & $08-14-91$ \\
\hline $30 \mathrm{~N} 31 \mathrm{~W} 10 \mathrm{ACBB} 03$ & 2,097 & $02-05-85$ & Qal & 78 & 2.0 & G & 75 & $\mathrm{U}$ & $08-14-91$ \\
\hline $30 \mathrm{~N} 31 \mathrm{~W} 10 \mathrm{ACBD} 01$ & 2,099 & $01-14-85$ & Qal & 71 & 2.0 & $G$ & 68 & $\mathbf{U}$ & $08-14-91$ \\
\hline 30N31W10ACBD02 & 2,099 & $01-14-85$ & Qal & 153 & 2.0 & $G$ & 150 & $\mathbf{U}$ & $08-14-91$ \\
\hline 30N31W10ACBD03 & 2,099 & $01-14-85$ & Qal & 230 & 2.0 & G & 227 & U & $08-14-91$ \\
\hline $30 \mathrm{~N} 31 \mathrm{~W} 10 \mathrm{DBCA01}$ & 2,120 & 10.15 .50 & $\mathrm{Qg}$ & 67 & 6.0 & - & - & $\mathbf{H}$ & $07-\quad-72$ \\
\hline $30 \mathrm{~N} 31 \mathrm{~W} 10 \mathrm{DBCA02}$ & 2,120 & $08-28-80$ & Qg & 71 & 6.0 & $\mathbf{P}$ & 65 & $\mathbf{H}$ & $08-15-91$ \\
\hline 30N31 W 10DDDB01 & 2,125 & $11-12-83$ & Qal & 90 & 2.0 & $G$ & 87 & $\mathbf{U}$ & $08-14-91$ \\
\hline 30N31W15AAAB01 & 2,130 & $12-\quad-50$ & Qal & 84 & 6.0 & - & -- & $\mathbf{H}$ & $08-15-91$ \\
\hline 30N31W15DCCCO1 & 2,435 & $05-07-74$ & Qg & 185 & 6.6 & $\mathbf{P}$ & 180 & H & 08-17-91 \\
\hline $30 \mathrm{~N} 31 \mathrm{~W} 22 \mathrm{AAB} \quad 01$ & 2,406 & $10-20-54$ & Qg & 170 & 4.0 & - & -- & H & 08-17-91 \\
\hline 30N31W23DAD 01 & 2,234 & $-\quad-50$ & Qal & 45 & 6.0 & - & -- & $\mathrm{H}$ & $07-\quad-72$ \\
\hline $30 \mathrm{~N} 31 \mathrm{~W} 23 \mathrm{DAD} \quad 02$ & 2,234 & $10-10-73$ & $\mathrm{Qg}$ & 96 & 6.0 & $\mathbf{P}$ & 91 & $H$ & $08-16-91$ \\
\hline $30 N 31 W 23 D A D 03$ & 2,234 & -. & Qal & 40 & -- & - & -- & $\mathbf{H}$ & -- \\
\hline $30 \mathrm{~N} 31 \mathrm{~W} 25 \mathrm{BCB} \quad 01$ & 2,272 & $08-18-67$ & Qal & 169 & 8.0 & $\mathbf{S}$ & 160 & HI & $08-18-91$ \\
\hline $30 \mathrm{~N} 31 \mathrm{~W} 25 \mathrm{BDB} \quad 01$ & 2,267 & $06-05-73$ & Qal & 170 & 8.0 & $\mathbf{P}$ & 135 & $\mathrm{U}$ & 08-16-91 \\
\hline $30 \mathrm{~N} 31 \mathrm{~W} 34 \mathrm{CAB} \quad 01$ & 2,497 & $08-16-64$ & Qal & 166 & 6.0 & $\mathbf{P}$ & 158 & $\mathbf{H}$ & $08-14-91$ \\
\hline 29N31 W02DDAB01 & 2,518 & $08-30-66$ & Qal & 157 & 6.0 & $\mathbf{P}$ & 153 & $\mathbf{H}$ & $08-13-91$ \\
\hline 29N31W02DDB 02 & 2,535 & $05-24-72$ & Qal & 337 & 6.0 & 0 & - & H & $08-15-91$ \\
\hline 29N31W10ADAD01 & 2,575 & $07-18-83$ & $\mathrm{Qg}$ & 360 & 6.0 & $\mathbf{P}$ & 157 & H & $08-18-91$ \\
\hline 29N31W13DAC 01 & 2,525 & $10-12-68$ & $\mathrm{Qg}$ & 465 & 6.0 & $\mathbf{P}$ & 461 & H & $08-14-91$ \\
\hline 29N30W06CBB 01 & 2,510 & 03-09-70 & Qg & 94 & 6.0 & $\mathbf{P}$ & 86 & $\mathbf{H}$ & $08-14-91$ \\
\hline 29N30W 18DBDA0 1 & 2,615 & $04-28-73$ & Qal & 200 & 6.0 & $\mathbf{P}$ & 196 & H & $08-20-91$ \\
\hline
\end{tabular}


Table 1. Records of selected wells by basin, Northern Rocky Mountains intermontane basins, Montana (Continued)

\begin{tabular}{|c|c|c|c|c|c|c|c|c|c|}
\hline $\begin{array}{c}\text { Water lovel } \\
\text { (ft) }\end{array}$ & $\begin{array}{c}\text { Dis- } \\
\text { charge } \\
\text { (gal/ } \\
\text { min) }\end{array}$ & $\begin{array}{c}\text { Spocific } \\
\text { capacity } \\
\text { [(gal/ } \\
\text { min)/fit] }\end{array}$ & $\begin{array}{l}\text { Source } \\
\text { of dis- } \\
\text { charge } \\
\text { data }\end{array}$ & $\begin{array}{l}\text { Type } \\
\text { of log } \\
\text { avall- } \\
\text { able }\end{array}$ & $\begin{array}{c}\text { Date } \\
\text { water- } \\
\text { quallty } \\
\text { parameter } \\
\text { measured }\end{array}$ & $\begin{array}{c}\text { Speciflc } \\
\text { conduc- } \\
\text { tance } \\
(\mu \mathrm{S} / \mathrm{cm})\end{array}$ & $\begin{array}{c}\text { pH } \\
\text { (stan- } \\
\text { dard } \\
\text { units) }\end{array}$ & $\begin{array}{l}\text { Tem- } \\
\text { per- } \\
\text { ature } \\
\left({ }^{\circ} \mathrm{C}\right)\end{array}$ & Well number \\
\hline \multicolumn{10}{|c|}{ Libby Creek Valley-Continued } \\
\hline 11.28 & 10 & 19 & $\mathbf{s}$ & - & $08-17-91$ & 220 & 7.3 & 8.0 & $31 \mathrm{~N} 31 \mathrm{~W} 33 \mathrm{CCBB} 01$ \\
\hline 121.60 & 10 & .8 & $\mathrm{D}$ & D & $08-19-91$ & 484 & 7.1 & 10.5 & $31 \mathrm{~N} 31 \mathrm{~W} 34 \mathrm{CCAC0} 1$ \\
\hline 208.39 & 10 & .5 & D & D & - & -- & -- & -- & 31N31W35AAAA01 \\
\hline 31.87 & 20 & 1.9 & D & D & $08-19-91$ & 485 & 6.8 & 10.5 & $31 \mathrm{~N} 31 \mathrm{~W} 35 \mathrm{CAD} 01$ \\
\hline 18.65 & -- & -. & - & - & $08-18-91$ & 920 & 7.2 & 10.0 & 31N31W35DDBA01 \\
\hline 15.88 & -- & -- & - & - & $08-14-91$ & -- & -- & 10.0 & 30N31W03CACD01 \\
\hline 14.63 & -- & -- & - & - & -. & -. & -- & -- & 30N31W03CACD02 \\
\hline 14.79 & -- & -- & - & - & -- & -- & -- & -- & 30N31W03CACD03 \\
\hline *230 & 3 & -- & D & D & $08-16-91$ & 277 & 7.2 & 12.5 & 30N31W08AAAB01 \\
\hline 21.73 & -- & -- & - & - & - & -- & -- & -- & 30N31W10ACAD01 \\
\hline 21.71 & -- & -- & - & - & - & - & -- & -- & 30N31 W 10ACAD02 \\
\hline 20.41 & -- & -- & - & D & -- & -- & -- & -- & 30N31W $10 A C B B 01$ \\
\hline 20.55 & -- & -- & - & D & -- & -- & -- & -- & 30N31W10ACBB02 \\
\hline 21.58 & -- & -- & - & D & -- & -- & -- & -- & $30 \mathrm{~N} 31 \mathrm{~W} 10 \mathrm{ACBB} 03$ \\
\hline 18.19 & -- & -- & - & D & -. & -- & -- & -- & $30 \mathrm{~N} 31 \mathrm{~W} 10 \mathrm{ACBD} 01$ \\
\hline 22.02 & -- & -- & - & - & -- & -- &.- & -- & 30N31 W10ACBD02 \\
\hline 21.74 & -- & -- & - & D & -- & -- & -- & -- & 30N31W10ACBD03 \\
\hline 30.60 & 31 & -- & D & D & 08-15-91 & 652 & 6.8 & 9.0 & 30N31W10DBCA01 \\
\hline 40.58 & 7 & 1.2 & $\mathbf{s}$ & D & $08-15-91$ & 690 & 6.7 & 10.0 & 30N31W 10DBCA02 \\
\hline 39.93 & -- & -- & - & D & -- & -- & - & - & $30 \mathrm{~N} 31 \mathrm{~W} 10 \mathrm{DDDB} 01$ \\
\hline 46.00 & 20 & .. & $\mathbf{S}$ & D & $08-15-91$ & 260 & 6.9 & 9.5 & 30N31W15AAAB01 \\
\hline 152.30 & 5 & -- & D & D & $08-17-91$ & 114 & 7.2 & 12.0 & 30N31W15DCCC01 \\
\hline 158.29 & 20 & -- & D & D & $08-02-73$ & 392 & -- & 15.0 & $30 \mathrm{~N} 31 \mathrm{~W} 22 \mathrm{AAB} \quad 01$ \\
\hline 15.10 & -- & -- & - & - & $07-09-73$ & 168 & -- & 14.0 & 30N31W23DAD 01 \\
\hline 44.38 & 10 & -- & $\mathrm{D}$ & $\mathrm{D}$ & $08-15-91$ & 198 & 7.1 & 10.5 & 30N31W23DAD 02 \\
\hline .- & .. & -- & - & - & $08-15-91$ & 227 & 6.4 & -- & $30 \mathrm{~N} 31 \mathrm{~W} 23 \mathrm{DAD} \quad 03$ \\
\hline 68.22 & 27 & .3 & D & D & $06-06-73$ & 422 & -- & 12.5 & $30 \mathrm{~N} 31 \mathrm{~W} 25 \mathrm{BCB} \quad 01$ \\
\hline 9.06 & 370 & 2.9 & D & D & -- & -- & -- & -- & $30 \mathrm{~N} 31 \mathrm{~W} 25 \mathrm{BDB} \quad 01$ \\
\hline 69.15 & 15 & .4 & D & D & $07-25-73$ & 491 & -- & 14.5 & $30 \mathrm{~N} 31 \mathrm{~W} 34 \mathrm{CAB} \quad 01$ \\
\hline 79.35 & 7 & .1 & R & D & $08-13-91$ & 408 & 7.3 & 13.0 & 29N31 W02DDAB01 \\
\hline 117.55 & 3 & -- & $\mathbf{R}$ & D & -- & -- & -- & -- & 29N31W02DDB 02 \\
\hline 115.62 & 4 & .2 & D & D & -- & -- & -- & -- & 29N31 W10ADAD01 \\
\hline 23.97 & 4 & -- & D & D & 08-14-91 & 330 & 7.8 & 10.5 & 29N3IW I3DAC 01 \\
\hline 50.39 & 10 & .4 & D & $\mathrm{D}$ & $07-29-73$ & 392 & -- & 11.0 & 29N30W06CBB 01 \\
\hline 23.50 & 30 & .8 & $\mathbf{R}$ & $\mathrm{D}$ & $08-20-91$ & 388 & 7.2 & 11.0 & 29N30W 18DBDA01 \\
\hline
\end{tabular}


Table 1. Records of selected wells by basin, Northern Rocky Mountains intermontane basins, Montana (Continued)

\begin{tabular}{|c|c|c|c|c|c|c|c|c|c|}
\hline Well number & $\begin{array}{l}\text { Altitude } \\
\text { of land } \\
\text { surface } \\
\text { (fi) }\end{array}$ & $\begin{array}{l}\text { Date wall } \\
\text { constructed }\end{array}$ & $\begin{array}{c}\text { Geologic } \\
\text { unit }\end{array}$ & $\begin{array}{c}\text { Depth } \\
\text { of } \\
\text { well } \\
\text { (fi) }\end{array}$ & $\begin{array}{c}\text { Dlam- } \\
\text { oter } \\
\text { of } \\
\text { caeling } \\
\text { (In.) }\end{array}$ & $\begin{array}{l}\text { Type } \\
\text { of } \\
\text { finish }\end{array}$ & $\begin{array}{l}\text { Top of } \\
\text { open } \\
\text { Inter- } \\
\text { val } \\
\text { (fi) }\end{array}$ & $\begin{array}{c}\text { Primary } \\
\text { use of } \\
\text { water }\end{array}$ & $\begin{array}{l}\text { Date water } \\
\text { lovel } \\
\text { meacured }\end{array}$ \\
\hline \multicolumn{10}{|c|}{ Libby Creek Valley-Continued } \\
\hline 29N30W31 ACBA01 & 2,700 & $09-29-88$ & Qal & 170 & 6.0 & $\mathbf{P}$ & 167 & $\mathbf{H}$ & $08-13-91$ \\
\hline 29N30W32BACC01 & 2,760 & $03-16-83$ & Qg & 209 & 6.0 & 0 & - & $\mathbf{H}$ & 08-13-91 \\
\hline 29N30W32BDBD01 & 2,820 & $08-30-76$ & Yms & 305 & 6.0 & $\mathbf{P}$ & 69 & $\mathbf{H}$ & $08-14-91$ \\
\hline \multicolumn{10}{|c|}{ Little Bitterroot Valley } \\
\hline 24N24W24ABBB01 & 2,860 & 09-22-83 & Qal & 69 & 2.0 & $\mathbf{P}$ & 37 & $\mathbf{U}$ & $07-10-91$ \\
\hline 24N24W24ABBB02 & 2,860 & 09-23-83 & Qal & 14 & 4.0 & $\mathbf{S}$ & 9 & $\mathbf{U}$ & $07-10-91$ \\
\hline 24N24W25DDBB01 & 2,859 & $10-05-84$ & Qal & 328 & 6.0 & $\mathbf{P}$ & 319 & $\mathbf{U}$ & $07-10-91$ \\
\hline 24N24W27ABDB01 & 2,840 & $10-07-84$ & Ts & 217 & 4.0 & $\mathbf{s}$ & 157 & $\mathbf{U}$ & $07-09-91$ \\
\hline 24N23W09BAA 01 & 2,960 & - & Qal & 170 & -- & - & -- & $\mathbf{U}$ & $07-10-91$ \\
\hline 24N23W21BCDA01 & 2,930 & -- & Qal & 250 & 6.0 & - & -- & $\mathbf{U}$ & $07-10-91$ \\
\hline $24 \mathrm{~N} 22 \mathrm{~W} 30 \mathrm{BCCCO1}$ & 3,153 & $10-17-84$ & Qal & 460 & 6.0 & $\mathbf{P}$ & 436 & $\mathbf{U}$ & $08-02-91$ \\
\hline 23N24W27CDDD01 & 2,800 & $-\quad-67$ & Qal & 184 & 4.0 & - & -- & $\mathbf{U}$ & $07-10-91$ \\
\hline 23N24W34ADAA01 & 2,879 & $02--41$ & Qal & 377 & 20.0 & $\mathbf{P}$ & 312 & $\mathbf{s}$ & $08-15-91$ \\
\hline 22N23W15DCDC01 & 2,835 & $09-29-84$ & Qal & 92 & 6.0 & $\mathbf{P}$ & 60 & $\mathbf{U}$ & $08-02-91$ \\
\hline 21N22W07DCAA01 & 2,800 & $09-28-84$ & Qal & 186 & 6.0 & $\mathbf{P}$ & 140 & $\mathbf{U}$ & $07-10-91$ \\
\hline 20N22W21CBDA01 & 2,750 & $09-24-84$ & Qal & 331 & 6.0 & $\mathbf{P}$ & 300 & $\mathbf{U}$ & 08-08-91 \\
\hline $20 \mathrm{~N} 22 \mathrm{~W} 28 \mathrm{ABCBO} 1$ & 2,740 & $09-20-84$ & Qal & 340 & 6.0 & $\mathbf{P}$ & 312 & $\mathbf{U}$ & 08-08-91 \\
\hline $20 \mathrm{~N} 22 \mathrm{~W} 28 \mathrm{ABCB} 02$ & 2,740 & 09-20-84 & Ts & 665 & 6.0 & $\mathbf{P}$ & 602 & $\mathbf{U}$ & $10-08-91$ \\
\hline 20N22W30DADD01 & 2,670 & $-\quad-69$ & Qal & 155 & 6.0 & o & -- & $\mathbf{U}$ & $08-08-91$ \\
\hline \multicolumn{10}{|c|}{ Lower Clark Fork Valley } \\
\hline 28N33W 10DCAB01 & 2,470 & $06-03-81$ & Qg & 80 & 6.0 & o & - & $\mathbf{H}$ & $08-17-91$ \\
\hline 27N35W 24DADA01 & 2,325 & $08-19-86$ & Qg & 185 & 6.0 & $\mathbf{P}$ & 175 & $\mathbf{H}$ & $09-20-91$ \\
\hline 27N34W28DCDA01 & 2,220 & $11--76$ & $\mathrm{Qg}$ & 131 & 6.0 & o & -- & H & 09-19-91 \\
\hline 27N34W30ADAD01 & 2,390 & $11-\quad-80$ & Qg & 260 & 6.0 & o & -- & $\mathbf{H}$ & $09-20-91$ \\
\hline 27N34W35DABA01 & 2,260 & $07-\quad-69$ & Qg & 132 & 8.0 & o & -- & $\mathbf{P}$ & $09-20-91$ \\
\hline 26N34W03CDBC01 & 2,445 & $08-22-86$ & Qg & 315 & 6.0 & - & - & H & $09-20-91$ \\
\hline 26N34W2ICCBD0I & 2,460 & $06-22-88$ & Qg & 198 & 6.0 & - & -- & $\mathbf{H}$ & $09-20-91$ \\
\hline 26N33W03CACD01 & 2,285 & $03-23-90$ & $\mathrm{Qg}$ & 145 & 6.0 & $\mathbf{O}$ & - & $\mathbf{H}$ & 09-19-91 \\
\hline 26N33W04CBAB01 & 2,361 & 09-04-80 & Qg & 397 & 6.0 & 0 & -- & $\mathbf{H}$ & - \\
\hline 26N33W25ADDA01 & 2,430 & $01-04-71$ & Qg & 279 & 6.0 & o & -- & $\mathbf{H}$ & $09-19-91$ \\
\hline 26N32W 19CDDB01 & 2,220 & $05-29-74$ & Qg & 73 & 10.0 & $\mathbf{P}$ & 63 & $\mathbf{P}$ & $05-29-74$ \\
\hline 26N32W30BABA01 & 2,330 & $06-05-74$ & Qg & 209 & 8.0 & o & -- & $\mathbf{P}$ & $06-05-74$ \\
\hline 25N32W03BAAB01 & 2,360 & $04-30-80$ & Qg & 197 & 6.0 & 0 & -- & $\mathbf{H}$ & $09-19-91$ \\
\hline 25N32W23ACAB01 & 2,439 & $12-31-66$ & Qg & 162 & 6.0 & o & -- & $\mathbf{H}$ & $09-19-91$ \\
\hline 24N31W05BCBA01 & 2,560 & 09-01-89 & Qg & 260 & 6.0 & o & -- & H & $09-18-91$ \\
\hline
\end{tabular}


Table 1. Records of selected wells by basin, Northern Rocky Mountains intermontane basins, Montana (Continued)

\begin{tabular}{|c|c|c|c|c|c|c|c|c|c|}
\hline $\begin{array}{l}\text { Water lovel } \\
\text { (ft) }\end{array}$ & $\begin{array}{c}\text { Dis- } \\
\text { charge } \\
\text { (gal/ } \\
\text { min) }\end{array}$ & $\begin{array}{c}\text { Spoclfic } \\
\text { capacity } \\
\text { [(gal/ } \\
\text { min)/ft] }\end{array}$ & $\begin{array}{c}\text { Source } \\
\text { of dis- } \\
\text { charge } \\
\text { data }\end{array}$ & $\begin{array}{l}\text { Type } \\
\text { of log } \\
\text { avell- } \\
\text { able }\end{array}$ & $\begin{array}{c}\text { Date } \\
\text { water- } \\
\text { quallty } \\
\text { parameter } \\
\text { measured }\end{array}$ & $\begin{array}{l}\text { Specific } \\
\text { conduc- } \\
\text { tance } \\
\text { ( } \mu \text { S/cm) }\end{array}$ & $\begin{array}{l}\text { pH } \\
\text { (stan- } \\
\text { dard } \\
\text { units) }\end{array}$ & $\begin{array}{l}\text { Tem- } \\
\text { per- } \\
\text { ature } \\
\left({ }^{\circ} \mathrm{C}\right)\end{array}$ & Well number \\
\hline
\end{tabular}

Libby Creek Valley-Continued

$\begin{array}{rrrrrrrrrr}10.57 & 8 & .1 & \text { D } & \text { D } & 08-13-91 & 118 & 6.9 & 10.0 & \text { 29N30W31ACBA01 } \\ 16.70 & 35 & .3 & \text { D } & \text { D } & 08-13-91 & 419 & 7.6 & 9.0 & \text { 29N30W32BACC01 } \\ 60.82 & 4 & .- & \text { D } & \text { D } & 08-13-91 & 415 & 7.6 & 11.0 & \text { 29N30W32BDBD01 }\end{array}$

\section{Little Bitterroot Valley}

$\begin{array}{rcc}7.02 & -- & -- \\ 7.34 & -- & -- \\ 73.65 & 51 & 18 \\ 28.80 & 9 & .1 \\ 5.70 & -- & -- \\ & & \\ 35.11 & -- & -- \\ 249.55 & 7 & 13 \\ 25.90 & -- & -- \\ 108.87 & 1,600 & 280 \\ 46.55 & 24 & 41 \\ & & \\ 31.71 & 64 & 33 \\ 105.84 & 37 & 30 \\ 91.46 & 46 & 24 \\ 91.66 & 11 & .1 \\ 3.13 & -- & --\end{array}$

\section{Lower Clark Fork Valley}

\begin{tabular}{|c|c|c|c|c|c|c|c|c|c|}
\hline 33.07 & 30 & .8 & $\mathrm{D}$ & D & -- & -. & -- & -- & 28N33W 10DCAB01 \\
\hline 160.50 & 14 & .9 & D & D & $09-20-91$ & 201 & 8.1 & 20.0 & 27N35W24DADA01 \\
\hline 55.28 & 40 & 13 & D & D & $09-19-91$ & 237 & 8.1 & 11.0 & $27 \mathrm{~N} 34 \mathrm{~W} 28 \mathrm{DCDA} 01$ \\
\hline 214.04 & 40 & 4.0 & D & $\mathrm{D}$ & $09-20-91$ & 194 & 8.2 & 8.0 & 27N34W30ADAD01 \\
\hline 81.02 & 33 & -- & $\mathrm{D}$ & D & $09-20-91$ & 260 & 8.1 & 10.5 & 27N34W35DABA01 \\
\hline 272.51 & 30 & .8 & D & D & $09-20-91$ & 223 & 8.3 & 8.5 & 26N34W03CDBC01 \\
\hline 15.78 & 24 & 100 & $\mathbf{S}$ & D & $09-20-91$ & 119 & 7.6 & 7.0 & $26 \mathrm{~N} 34 \mathrm{~W} 21 \mathrm{CCBD} 01$ \\
\hline 129.10 & 10 & .8 & D & D & .. & -- & -- & - & 26N33W03CACD01 \\
\hline -- & 100 & -- & D & D & $09-20-91$ & 242 & 8.2 & 9.0 & 26N33W04CBAB01 \\
\hline 252.44 & 10 & 1.7 & D & D & 09-19-91 & 234 & 8.3 & 8.0 & 26N33W25ADDA01 \\
\hline *41 & 180 & -- & D & D & -. & -- & -- & - & $26 \mathrm{~N} 32 \mathrm{~W} 19 \mathrm{CDDB} 01$ \\
\hline$*^{*} 155$ & 180 & -. & D & D & $09-19-91$ & 120 & 8.5 & 7.5 & 26N32W30BABA01 \\
\hline 134.75 & 20 & 1.5 & D & D & $09-19-91$ & 181 & 8.0 & 14.0 & 25N32W03BAABOI \\
\hline 105.25 & 20 & 10 & D & D & 09-19-91 & 95 & 8.7 & 8.0 & 25N32W $23 \mathrm{ACAB01}$ \\
\hline 232.28 & 20 & 1.7 & $\mathrm{D}$ & D & $09-18-91$ & 235 & 8.4 & 9.5 & 24N31W05BCBA01 \\
\hline
\end{tabular}


Table 1. Records of selected wells by basin, Northern Rocky Mountains intermontane basins, Montana (Continued)

\begin{tabular}{|c|c|c|c|c|c|c|c|c|c|}
\hline Woll number & $\begin{array}{c}\text { Alttude } \\
\text { of land } \\
\text { surface } \\
\text { (ft) }\end{array}$ & $\begin{array}{c}\text { Date well } \\
\text { constructed }\end{array}$ & $\begin{array}{c}\text { Geologic } \\
\text { unit }\end{array}$ & $\begin{array}{c}\text { Depth } \\
\text { of } \\
\text { well } \\
\text { (ft) }\end{array}$ & $\begin{array}{c}\text { Dlam- } \\
\text { etor } \\
\text { of } \\
\text { casing } \\
\text { (in.) }\end{array}$ & $\begin{array}{c}\text { Type } \\
\text { of } \\
\text { finlsh }\end{array}$ & $\begin{array}{l}\text { Top of } \\
\text { open } \\
\text { Inter- } \\
\text { val } \\
\text { (ft) }\end{array}$ & $\begin{array}{l}\text { Primary } \\
\text { uee of } \\
\text { water }\end{array}$ & $\begin{array}{l}\text { Date water } \\
\text { level } \\
\text { measured }\end{array}$ \\
\hline \multicolumn{10}{|c|}{ Lower Clark Fork Valley_Continued } \\
\hline 24N3IW17DBAA01 & 2,355 & $04-06-83$ & Qg & 60 & 6.0 & $\mathbf{0}$ & -- & c & $09-19-91$ \\
\hline $24 \mathrm{~N} 31 \mathrm{~W} 17 \mathrm{DDDC} 01$ & 2,369 & $04-26-42$ & Qg & 208 & 12.0 & $\mathbf{P}$ & 188 & $\mathbf{P}$ & $05-01-42$ \\
\hline 24N30W3IDDAB01 & 2,540 & $01-12-77$ & Qg & 220 & 6.0 & $\mathbf{O}$ & -- & $\mathbf{H}$ & $09-18-91$ \\
\hline $23 \mathrm{~N} 30 \mathrm{~W} 20 \mathrm{DCCCO} 1$ & 2,567 & $08-03-67$ & Yms & 170 & 6.0 & $\mathbf{x}$ & 155 & $\mathbf{H}$ & $09-16-91$ \\
\hline $23 \mathrm{~N} 30 \mathrm{~W} 20 \mathrm{DCDCO} 1$ & 2,569 & -65 & Qg & 16 & 3.0 & - & - & $\mathbf{U}$ & $09-16-91$ \\
\hline 23N30W35DCAB01 & 2,610 & $07-22-81$ & Yms & 300 & 6.0 & $\mathbf{P}$ & 250 & $\mathbf{H}$ & $09-18-91$ \\
\hline 22N30W23BDDA01 & 2,400 & $01-15-90$ & Qg & 32 & 6.0 & $\mathbf{O}$ & -- & $\mathbf{H}$ & $09-18-91$ \\
\hline 22N30W23DBAB01 & 2,401 & $08-15-72$ & Qg & 97 & 8.0 & o & - & $\mathbf{H}$ & $09-18-91$ \\
\hline $22 \mathrm{~N} 30 \mathrm{~W} 24 \mathrm{CCCCO1}$ & 2,403 & $03-21-79$ & Qg & 90 & 6.0 & o & -- & $\mathbf{H}$ & $09-17-91$ \\
\hline $22 \mathrm{~N} 29 \mathrm{~W} 33 \mathrm{CCCCO} 1$ & 2,757 & $06-28-89$ & Yms & 300 & 6.6 & $\mathbf{P}$ & 260 & $\mathbf{H}$ & $09-17-91$ \\
\hline 2IN30W I4AAAD0I & 2,685 & $07--71$ & Qg & 172 & 6.0 & $\mathbf{o}$ & - & $\mathbf{H}$ & $09-17-91$ \\
\hline 21 N29W09BDDC01 & 2,403 & $04--65$ & Qg & -- & -- & - & -- & $\mathbf{P}$ & -- \\
\hline $21 \mathrm{~N} 29 \mathrm{~W} 09 \mathrm{BDDC02}$ & 2,403 & $01-11-83$ & Qg & 51 & 16.0 & $\mathbf{s}$ & 41 & $\mathbf{P}$ & $09-17-91$ \\
\hline 21N29W09DDCB01 & 2,421 & $06 \cdot 12-74$ & Yms & 250 & 6.0 & $\mathrm{x}$ & 191 & $\mathbf{H}$ & $09-17-91$ \\
\hline $21 \mathrm{~N} 29 \mathrm{~W} 23 \mathrm{CBDC01}$ & 2,528 & $07-11-79$ & Qg & 180 & 6.0 & o & - & $\mathbf{H}$ & $09-17-91$ \\
\hline \multicolumn{10}{|c|}{ Madison River Valley } \\
\hline 04S02W01CACC01 & 5,545 & $04-17-80$ & Ts & 71 & 6.0 & $\mathbf{P}$ & 65 & $\mathbf{H}$ & $09-11-91$ \\
\hline 04S02W36CAD 01 & 5,275 & $06-25-74$ & Ts & 125 & 6.0 & $\mathbf{P}$ & 52 & $\mathbf{U}$ & $09-11-91$ \\
\hline 04S01W30BA 01 & 5,075 & $10-14-80$ & Ts & 101 & 6.0 & $\mathbf{o}$ & -- & $\mathbf{H}$ & $09-15-91$ \\
\hline 04S01W35AADD01 & 4,830 & $03-30-82$ & Ts & 129 & 6.0 & o & -- & $\mathbf{H}$ & $09-12-91$ \\
\hline 05S0IW04BA^B01 & 4,905 & $04-20-92$ & Qal & 38 & 6.0 & $\mathbf{O}$ & -- & $\mathbf{H}$ & $09-24-92$ \\
\hline 05S01W04DDDB01 & 4,900 & $05-01-85$ & Qal & 117 & 6.0 & $\mathbf{o}$ & - & $\mathrm{H}$ & $09-12-91$ \\
\hline 05SOIWI7AAAA0I & 4,935 & $04-30-82$ & Ts & 193 & 6.0 & $\mathbf{P}$ & 27 & $\mathbf{S}$ & $09-12-91$ \\
\hline 05SOIW21 CCCCO1 & 4,970 & $05-28-65$ & Qal & 33 & 6.0 & o & -- & $\mathbf{S}$ & $09-22-92$ \\
\hline 05S01W23CCBDO1 & 4,870 & -.79 & Unk & 876 & -- & - & -- & $\mathbf{U}$ & - \\
\hline 05S0IW23DABA0I & 4,890 & $05-28-80$ & Qal & 44 & 6.0 & $\mathbf{P}$ & 35 & $\mathbf{H}$ & $09-12-91$ \\
\hline 05S01W28BCAB01 & 4,910 & .- & Qal & 64 & 10.5 & - & -- & $\mathbf{s}$ & $09-22-92$ \\
\hline 05S01W28DBDD01 & 4,925 & $08-12 \cdot 82$ & KAm & 956 & 8.0 & $\mathbf{x}$ & 474 & c & - \\
\hline 05S01 W33CBCBO1 & 4,950 & $11-22-66$ & Qal & 84 & 6.0 & $\mathbf{o}$ & -- & $\mathbf{P}$ & $09-11-91$ \\
\hline 05S01E28CDDD01 & 5,290 & -- & Qal & 115 & 6.0 & - & -- & $\mathbf{H}$ & $09-12-91$ \\
\hline 05S01E32ACCBO1 & 5,368 & $10-15-75$ & Ts & 393 & 8.0 & $\mathbf{o}$ & - & $\mathbf{S}$ & $09-12-91$ \\
\hline 05S01E34BDCB01 & 5,475 & $05-24-84$ & Yms & 425 & 6.0 & $\mathbf{P}$ & 160 & H & $09-13-91$ \\
\hline 06S02W13CDCD01 & 5,370 & $06-04-84$ & Ts & 83 & 6.0 & o & -- & D & $09-26-91$ \\
\hline 06S02W24BACD01 & 5,380 & $04-06-89$ & Ts & 341 & 6.0 & o & -- & $\mathbf{H}$ & $09-27-91$ \\
\hline 06S02W36DAAD01 & 5,190 & $12-24-73$ & Ts & 100 & 6.0 & o & -- & H & $09-27-91$ \\
\hline 06S01W04ACCB01 & 4,960 & $08-24-60$ & Ts & 165 & 8.0 & $\mathbf{P}$ & 100 & $\mathbf{P}$ & $08-24-60$ \\
\hline 06S01 W04BCAD01 & 4,970 & $09-16-79$ & Qal & 142 & 12.0 & $\mathbf{P}$ & 75 & $\mathbf{P}$ & $09-16-79$ \\
\hline 06S0IW05ACBD01 & 5,095 & $09-28-87$ & Ts & 240 & 6.0 & $\mathbf{P}$ & 210 & $\mathbf{H}$ & $09-10-91$ \\
\hline 06S01W06DDA 01 & 5,245 & $08-11-77$ & Ts & 228 & 6.0 & $\mathbf{P}$ & 222 & H & $09-11-91$ \\
\hline 06S01W08CADD01 & 5,145 & 09-05-85 & Ts & 162 & 6.0 & o & - & H & -- \\
\hline 06S01W08DABD01 & 5,010 & $04-10-88$ & Qal & 50 & 6.0 & $\mathbf{P}$ & 40 & $\mathbf{H}$ & $09-24-91$ \\
\hline
\end{tabular}


Table 1. Records of selected wells by basin, Northern Rocky Mountains intermontane basins, Montana (Continued)

\begin{tabular}{|c|c|c|c|c|c|c|c|c|c|}
\hline $\begin{array}{c}\text { Water level } \\
\text { (ft) }\end{array}$ & $\begin{array}{c}\text { Dis- } \\
\text { charge } \\
\text { (gal/ } \\
\text { mln) }\end{array}$ & $\begin{array}{c}\text { Specific } \\
\text { capacity } \\
{[(g a l} \\
\text { min)/ft] }\end{array}$ & $\begin{array}{l}\text { Source } \\
\text { of dls- } \\
\text { charge } \\
\text { data }\end{array}$ & $\begin{array}{l}\text { Type } \\
\text { of log } \\
\text { avall- } \\
\text { able }\end{array}$ & $\begin{array}{c}\text { Date } \\
\text { water- } \\
\text { quallty } \\
\text { peramater } \\
\text { measured }\end{array}$ & $\begin{array}{l}\text { Specific } \\
\text { conduc- } \\
\text { tance } \\
(\mu S / c m)\end{array}$ & $\begin{array}{c}\text { pH } \\
\text { (stan- } \\
\text { dard } \\
\text { units) }\end{array}$ & $\begin{array}{l}\text { Tem- } \\
\text { per- } \\
\text { ature } \\
\left({ }^{\circ} \mathrm{C}\right)\end{array}$ & Well number \\
\hline
\end{tabular}

Lower Clark Fork Valley-Continued

\begin{tabular}{|c|c|c|c|c|c|c|c|c|c|}
\hline 28.70 & 40 & 3.6 & $\mathrm{D}$ & $\mathrm{D}$ & $09-19-91$ & 302 & 8.0 & 8.0 & 24N31 WI7DBAA0I \\
\hline *35 & 550 & 31 & $\mathrm{D}$ & - & -- & -- & -- & -- & 24N31 W 17DDDC01 \\
\hline 170.98 & 7 & .7 & $\mathbf{D}$ & D & $09-18-91$ & 250 & 7.7 & 10.5 & 24N30W31DDABO1 \\
\hline 134.91 & 20 & -- & $\mathbf{D}$ & D & $09-16-91$ & 494 & 7.6 & 9.5 & 23N30W20DCCC01 \\
\hline 3.77 & -- & -- & - & - & -- & - & - & -- & 23N30W20DCDC01 \\
\hline 60.26 & 5 & .4 & $\mathbf{D}$ & D & -- & - & -- & -- & 23N30W35DCAB01 \\
\hline 22.78 & 20 & 6.7 & $\mathbf{D}$ & $\mathrm{D}$ & $09-18-91$ & 390 & 7.5 & 8.5 & $22 \mathrm{~N} 30 \mathrm{~W} 23 \mathrm{BDDA0} 1$ \\
\hline 63.97 & 13 & 58 & $\mathbf{S}$ & D & 09.18 .91 & 388 & 8.0 & 9.5 & 22N30W23DBAB01 \\
\hline 63.94 & 40 & 4.0 & $\mathbf{D}$ & D & $09-17-9 !$ & 323 & 7.9 & 9.5 & $22 \mathrm{~N} 30 \mathrm{~W} 24 \mathrm{CCCC} 01$ \\
\hline 186.83 & 30 & 3.8 & $\mathbf{D}$ & $\mathbf{D}$ & $09-17-91$ & 747 & 7.6 & 11.5 & $22 \mathrm{~N} 29 \mathrm{~W} 33 \mathrm{CCCC} 01$ \\
\hline 99.53 & 25 & .4 & D & D & $09-17-91$ & 178 & 8.4 & 8.5 & 21N30W14AAAD0 1 \\
\hline-- & -- & -- & - & - & $09-17-91$ & 439 & 7.4 & 9.5 & 21N29W09BDDC01 \\
\hline 11.53 & 1,500 & 470 & $\mathbf{D}$ & D & -- & -- & -- & -- & 21N29W09BDDC02 \\
\hline 32.27 & 20 & .3 & $\mathbf{D}$ & $\mathrm{D}$ & $09-17-91$ & 402 & 7.9 & 14.5 & 21N29W09DDCB01 \\
\hline 138.24 & 30 & 1.3 & $\mathbf{D}$ & $\mathbf{D}$ & $09-17-91$ & 217 & 8.3 & 8.0 & $21 \mathrm{~N} 29 \mathrm{~W} 23 \mathrm{CBDC0} 1$ \\
\hline
\end{tabular}

\section{Madison River Valley}

\begin{tabular}{|c|c|c|c|c|c|c|c|c|c|}
\hline 27.60 & 15 & .9 & D & D & $09-11-91$ & 180 & - & 10.0 & 04S02W01CACC01 \\
\hline 9.07 & 400 & 100 & D & D & -- & -- & -- & -- & 04S02W36CAD 01 \\
\hline 43.09 & 12 & -- & D & D & $09-15-91$ & 300 & 7.4 & 11.0 & 04S01W30BA \\
\hline 20.84 & 5 & .1 & D & D & - & -- & -- & -- & 04S01 W35AADD01 \\
\hline 10.85 & 25 & 3.1 & D & D & 09-24-92 & 424 & 7.3 & 10.0 & 05S01W04BAAB01 \\
\hline 71.77 & 30 & .7 & D & D & 09-12-91 & 283 & 8.0 & 11.5 & 05S01 W04DDDB0! \\
\hline 3.30 & 6 & .5 & D & D & -- & -- & - & -- & 05S01W!7AAAA01 \\
\hline 16.54 & -. & -. & - & D & -- & -- & -- & - & 05S01 W21CCCC01 \\
\hline -- & -- & -- & - & - & -- & -- & -- & -- & 05S01W23CCBD01 \\
\hline 21.80 & 30 & 1.6 & D & D & $09-12-91$ & 337 & 7.1 & 10.0 & 05S01 W23DABA01 \\
\hline 7.39 & 10 & 1.1 & $\mathbf{S}$ & - & 09-22-92 & 563 & 7.9 & 14.0 & 05S01 W28BCAB01 \\
\hline$-\infty$ & 75 & - & $\mathbf{A}$ & D & $09-24-91$ & -- & - & 89.5 & 05S01W28DBDD01 \\
\hline 9.24 & 30 & .4 & D & D & $09-11-91$ & 248 & 7.9 & 13.0 & 05S01 W33CBCB01 \\
\hline 69.79 & -- & -- & - & - & $09-12-91$ & 340 & -- & 9.0 & 05S0 IE28CDDD01 \\
\hline 241.39 & 15 & .1 & D & D & -- & -- & -- & - & 05SOIE32ACCB01 \\
\hline 113.05 & 2 & -- & D & D & $09-13-91$ & 280 & - & 10.5 & 05S01E34BDCB01 \\
\hline 52.88 & 30 & 1.5 & D & D & $09-26-91$ & 426 & -- & 10.5 & 06S02W13CDCD01 \\
\hline 63.78 & 10 & .1 & D & D & $10-27-91$ & 417 & 7.1 & 14.0 & 06S02W24BACD01 \\
\hline 5.68 & 10 & .1 & D & D & 09-27-91 & 461 & -- & 10.0 & 06S02W36DAAD01 \\
\hline$* 13$ & 200 & 2.9 & D & D & -- & -- & -- & -- & 06S01W04ACCB01 \\
\hline$* 28$ & 400 & 5.2 & D & D & -- & -- & $m$ & - & 06S01 W04BCAD01 \\
\hline 119.99 & -- & -- & - & D & $09-10-91$ & 736 & - & 16.0 & 06S01 W05ACBD01 \\
\hline 122.67 & 5 & .1 & D & D & $09-11-9 \mid$ & 774 & -- & 12.5 & 06S01W06DDA 01 \\
\hline- & 13 & .1 & D & D & $09-22-92$ & 443 & 7.9 & 11.0 & 06S01 W08CADD0I \\
\hline 15.85 & 8 & 3.7 & $\mathbf{s}$ & D & $09-24-91$ & 374 & - & 13.5 & 06S01 W08DABD01 \\
\hline
\end{tabular}


Table 1. Records of selected wells by basin, Northern Rocky Mountains intermontane basins, Montana (Continued)

\begin{tabular}{|c|c|c|c|c|c|c|c|c|c|}
\hline Well number & $\begin{array}{l}\text { Altitude } \\
\text { of land } \\
\text { surfacs } \\
\text { (ft) }\end{array}$ & $\begin{array}{l}\text { Date well } \\
\text { constructed }\end{array}$ & $\begin{array}{c}\text { Geologic } \\
\text { unit }\end{array}$ & $\begin{array}{l}\text { Depth } \\
\text { of } \\
\text { wsll } \\
\text { (ft) }\end{array}$ & $\begin{array}{c}\text { Dlam- } \\
\text { oter } \\
\text { of } \\
\text { casing } \\
\text { (in.) }\end{array}$ & $\begin{array}{l}\text { Type } \\
\text { of } \\
\text { finlsh }\end{array}$ & $\begin{array}{l}\text { Top of } \\
\text { open } \\
\text { Inter- } \\
\text { val } \\
\text { (fi) }\end{array}$ & $\begin{array}{l}\text { Primary } \\
\text { use of } \\
\text { walor }\end{array}$ & $\begin{array}{l}\text { Date water } \\
\text { lovel } \\
\text { measured }\end{array}$ \\
\hline \multicolumn{10}{|c|}{ Madison River Valley-Continued } \\
\hline 06S01 W23BBAC01 & 5,095 & $07-09-91$ & Qal & 37 & 6.0 & $\mathbf{o}$ & - & $\mathbf{H}$ & -- \\
\hline 06S01W23BBBA01 & 5,090 & $07-05-85$ & Ts & 170 & 6.0 & $\mathbf{O}$ & -- & $\mathbf{H}$ & $09-16-91$ \\
\hline 06S01W35DAAB01 & 5,200 & $03-25-64$ & Ts & 73 & 6.0 & o & -- & $\mathbf{U}$ & 09-16-91 \\
\hline 06S01E31CAAC01 & 5,330 & $04-27-90$ & Ts & 168 & 6.0 & $\mathbf{P}$ & 159 & $\mathbf{H}$ & 09-12-91 \\
\hline 07S02W24CCBD01 & 5,610 & $10-17-75$ & Ts & 100 & 6.0 & $\mathbf{P}$ & 80 & H & $09-27-91$ \\
\hline 07S01W03CAB 01 & 5,260 & $07-24-65$ & Ts & 138 & 6.0 & o & - & $\mathbf{U}$ & 09-16-91 \\
\hline 07S01W06BBAC01 & 5,155 & $-\quad-59$ & Ts & 72 & 6.0 & - & -- & $\mathbf{H}$ & - \\
\hline 07S01 W07DBCD01 & 5,300 & $10-06-81$ & Ts & 204 & 6.0 & 0 & -- & $\mathbf{H}$ & $09-25-91$ \\
\hline 07SO1W12DBBC01 & 5,285 & -. & Ts & -- & 6.0 & - & -- & $\mathbf{H}$ & -- \\
\hline 07S01 W18DDAC01 & 5,260 & $06-18-84$ & QaI & 71 & 6.0 & $\mathbf{P}$ & .51 & $\mathbf{H}$ & $09-24-91$ \\
\hline 07S01W20CBAA01 & 5,240 & $-\quad-50$ & Qal & 68 & 6.0 & - & -- & H & $09-21-92$ \\
\hline 07S01 W23ABBA01 & 5,335 & $08-2.5-61$ & Ts & 175 & 6.0 & O & -- & U & 09-16-91 \\
\hline 07S01W26AAAA01 & 5,375 & $10-29-70$ & Ts & 122 & 6.0 & O & -. & $\mathbf{P}$ & $10-29-70$ \\
\hline 07S01W34AAC 01 & 5,415 & $05-13-64$ & Ts & 225 & 6.0 & o & -- & $\mathbf{U}$ & $09-25-91$ \\
\hline 08SO1W25BBC 01 & 5,450 & $12-17-82$ & Qal & 80 & 6.0 & $\mathbf{O}$ & -- & $\mathbf{H}$ & $09-17-91$ \\
\hline 08S01W34DDDB01 & 5,745 & $09-18-88$ & Ts & 250 & 6.0 & $\mathbf{P}$ & 110 & $\mathbf{N}$ & $09-17-91$ \\
\hline 08S0 1E03CABB01 & 5,865 & $07-18-77$ & Ts & 213 & 6.0 & $\mathbf{S}$ & 201 & $\mathbf{H}$ & 09-13-91 \\
\hline 08S01E27DBAD01 & 5,890 & $12-03-81$ & Ts & 140 & 6.0 & $\mathbf{s}$ & 57 & H & 09-13-91 \\
\hline 09S01W01DBBA01 & 5,500 & $02-16-82$ & Ts & 255 & 12.0 & $\mathbf{P}$ & 100 & $\mathrm{U}$ & $09-17-91$ \\
\hline 09S01W12DCAD01 & 5,525 & $08-11-65$ & Qal & 47 & 6.0 & $\mathbf{O}$ & -- & $\mathbf{R}$ & $09-14-91$ \\
\hline 09S01E07CBAC01 & 5,675 & $06-28-89$ & Ts & 239 & 6.0 & 0 & - & $\mathbf{H}$ & $09-17-91$ \\
\hline 09S01E27CACD01 & 6,320 & $10-22-87$ & Ts & 220 & 6.0 & o & -- & $\mathbf{H}$ & $09-26-91$ \\
\hline 09S01E31BCAB01 & 5,700 & $10-01-80$ & QTKe & 138 & 6.0 & $\mathbf{P}$ & 90 & $\mathbf{U}$ & $09-18-91$ \\
\hline 10S01E06BBBD01 & 5,640 & $08-08-66$ & Qal & 70 & 6.0 & o & - & $\mathbf{U}$ & $09-14-91$ \\
\hline 10S01E06BBCC 01 & 5,660 & $08-03-66$ & Qal & 59 & 6.0 & $\mathbf{O}$ & - & $\mathbf{U}$ & $09-14-91$ \\
\hline 10S01E15DDAD01 & 6,435 & 08-25-59 & Ts & 105 & 6.0 & $\mathbf{P}$ & 76 & $\mathbf{H}$ & $09-25-91$ \\
\hline 10S01E21CCDDOI & 5,805 & $08-20-82$ & Ts & 400 & 6.0 & $\mathbf{P}$ & 240 & $\mathbf{U}$ & $09-26-91$ \\
\hline $11 \mathrm{~S} 01 \mathrm{E} 03 \mathrm{CACB} 01$ & 5,870 & $08-26-66$ & Qal & 97 & 6.0 & - & -- & $\mathbf{U}$ & $09-14-91$ \\
\hline 11S01E03CCCB01 & 5,895 & $11-23-83$ & Qal & 107 & 6.0 & o & -- & H & $09-16-91$ \\
\hline 11S01E10DACD01 & 5,875 & -- & Qal & 73 & 6.0 & - & - & $\mathbf{U}$ & $09-16-91$ \\
\hline 11S01E14BABD01 & 5,925 & $10-05-82$ & Ts & 185 & 6.0 & $\mathbf{P}$ & 165 & $\mathbf{P}$ & $09-16-91$ \\
\hline $11 \mathrm{S02E30BDCD01}$ & 6,135 & $04-21-81$ & Qal & 140 & 6.0 & $\mathbf{0}$ & $\cdots$ & $\mathbf{U}$ & $09-25-91$ \\
\hline 12S02E03ABDB01 & 6,205 & $05-31-91$ & Qal & 54 & 6.0 & 0 & -- & $\mathbf{H}$ & $09-25-91$ \\
\hline 12S02E34DAD 01 & 6,885 & $07-25-90$ & Pzls & 560 & 8.0 & $\mathbf{P}$ & 520 & $\mathbf{H}$ & - \\
\hline \multicolumn{10}{|c|}{ Mission Valley } \\
\hline 23N22W26BDCC 01 & 2,963 & $10-18-84$ & Qal & 80 & 6.0 & $\mathbf{P}$ & 40 & U & $10-08-91$ \\
\hline $23 \mathrm{~N} 21 \mathrm{~W} 19 \mathrm{CBC} \quad 01$ & 3,680 & $-\quad-73$ & Yms & 225 & -. & - & -- & $\mathbf{U}$ & $10-06-88$ \\
\hline 23N21W23BCAA01 & 3,435 & 03-10-74 & Yms & 300 & 6.6 & $\mathbf{P}$ & 180 & $\mathbf{u}$ & 08-01-91 \\
\hline 23N21W25DAAA01 & 3,060 & $04-16-73$ & Qg & 138 & 6.0 & - & - & $\mathbf{H}$ & $07-24-91$ \\
\hline 23N21 W34AAAA01 & 3,326 & $02-02-59$ & Qg & 342 & 6.0 & o & - & $\mathbf{H}$ & $07-24-91$ \\
\hline
\end{tabular}


Table 1. Records of selected wells by basin, Northern Rocky Mountains intermontane basins, Montana (Continued)

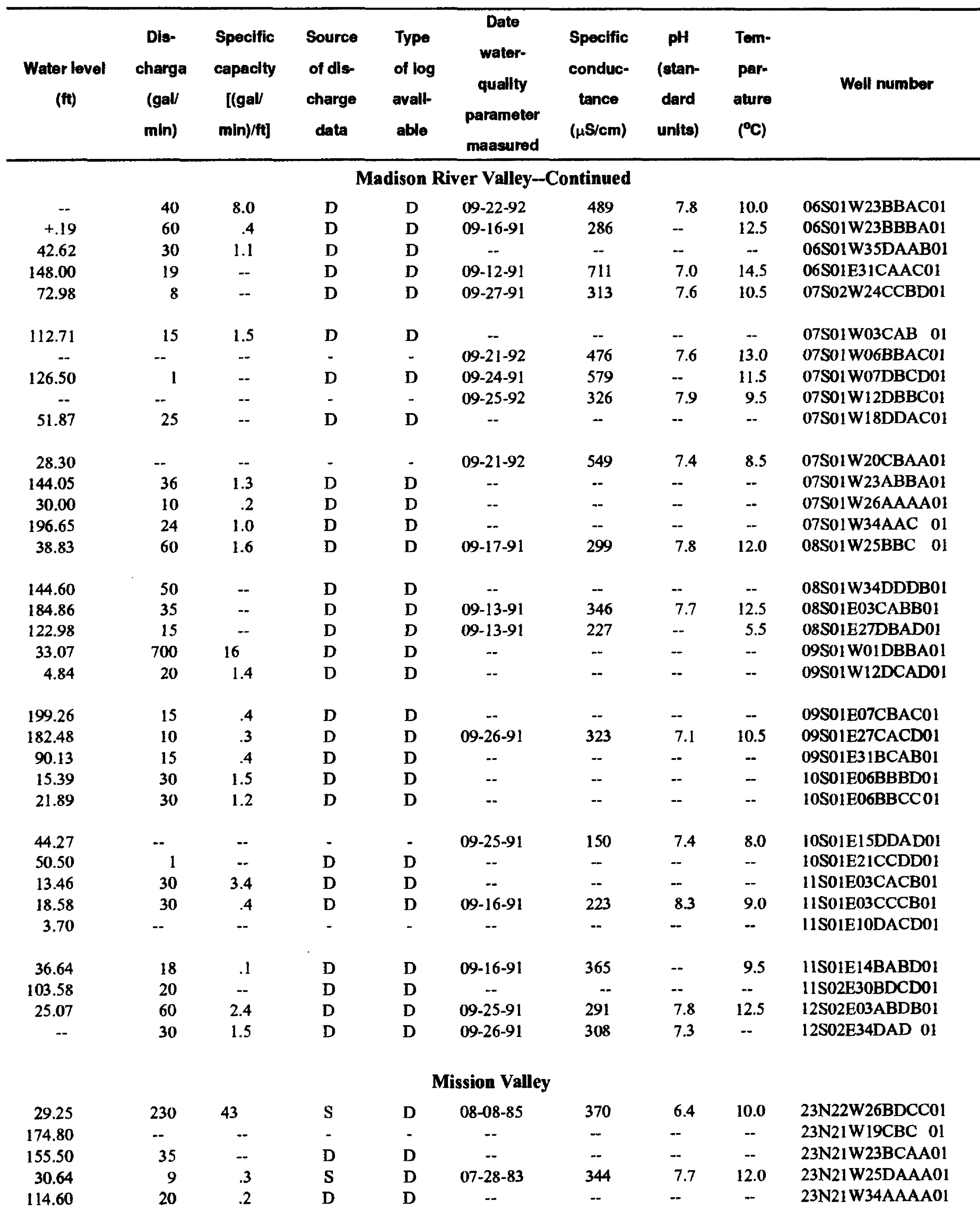


Table 1. Records of selected wells by basin, Northern Rocky Mountains intermontane basins, Montana (Continued)

\begin{tabular}{|c|c|c|c|c|c|c|c|c|c|}
\hline Well number & $\begin{array}{l}\text { Altitude } \\
\text { of land } \\
\text { surface } \\
\text { (ft) }\end{array}$ & $\begin{array}{l}\text { Date well } \\
\text { constructed }\end{array}$ & $\begin{array}{c}\text { Geologic } \\
\text { unit }\end{array}$ & $\begin{array}{l}\text { Depth } \\
\text { of } \\
\text { well } \\
\text { (ft) }\end{array}$ & $\begin{array}{c}\text { Diam- } \\
\text { oter } \\
\text { of } \\
\text { casing } \\
\text { (in.) }\end{array}$ & $\begin{array}{c}\text { Type } \\
\text { of } \\
\text { finlsh }\end{array}$ & $\begin{array}{c}\text { Top of } \\
\text { open } \\
\text { Inter- } \\
\text { vel } \\
\text { (ft) }\end{array}$ & $\begin{array}{c}\text { Primary } \\
\text { use of } \\
\text { water }\end{array}$ & $\begin{array}{l}\text { Date wator } \\
\text { lovel } \\
\text { measured }\end{array}$ \\
\hline \multicolumn{10}{|c|}{ Mission Valley-Continued } \\
\hline 22N22W04ABA 01 & 3,035 & -- & Qg & 440 & -- & - & -- & $\mathbf{U}$ & $08-08-91$ \\
\hline $22 \mathrm{~N} 22 \mathrm{~W} 26 \mathrm{DDDD} 01$ & 2,650 & $10-19-84$ & Qal & 50 & 6.0 & $\mathbf{P}$ & 20 & $\mathbf{U}$ & 08-08-91 \\
\hline 22N21W28DDDC01 & 2,936 & -. & Qg & - & -- & - & -- & $\mathbf{U}$ & $10-08-91$ \\
\hline 22N21W28DDDD01 & 2,935 & .. & Yms & 600 & -- & - & -- & $\mathrm{U}$ & $10-08-91$ \\
\hline 22N20W23DAD 01 & 3,220 & $-\quad-49$ & $\mathrm{Qg}$ & 500 & 6.0 & - & -- & $\mathbf{U}$ & $08-02-91$ \\
\hline $21 \mathrm{~N} 22 \mathrm{~W} 36 \mathrm{BDCC} 01$ & 2,615 & $10-27-84$ & Qal & 103 & 6.0 & $\mathbf{P}$ & 37 & $\mathbf{U}$ & 08-08-91 \\
\hline $21 \mathrm{~N} 22 \mathrm{~W} 36 \mathrm{BDCC} 02$ & 2,615 & $10-28-84$ & Qal & 99 & 6.0 & $\mathbf{P}$ & 80 & $\mathbf{U}$ & 08-08-91 \\
\hline $21 \mathrm{~N} 21 \mathrm{~W} 35 \mathrm{CCC} 01$ & 3,120 & $-\quad-53$ & $\mathrm{Qg}$ & 600 & 6.0 & - & -- & $\mathbf{H}$ & $08-01-91$ \\
\hline $21 \mathrm{~N} 21 \mathrm{~W} 36 \mathrm{CBA} 01$ & 3,080 & $-\quad-14$ & Qg & 420 & -- & - & -- & $\mathrm{U}$ & $08-01-91$ \\
\hline $21 \mathrm{~N} 20 \mathrm{~W} 14 \mathrm{ACB} 01$ & 3,045 & $-\quad-10$ & $\mathrm{Qg}$ & 12 & 44.0 & o & -- & $\mathbf{S}$ & 08-01-91 \\
\hline $21 \mathrm{~N} 20 \mathrm{~W} 24 \mathrm{CAAA} 02$ & 3,070 & -74 & Qg & 290 & 8.0 & - & $\cdots$ & $\mathrm{U}$ & 08-01-91 \\
\hline $21 \mathrm{~N} 20 \mathrm{~W} 31 \mathrm{AABB} 01$ & 3,060 & - & Qg & $\cdots$ & -- & - & -- & $\mathbf{U}$ & 08-01-91 \\
\hline 20N20W26CCBD0 1 & 3,050 & $-\quad-67$ & Qg & 200 & 6.0 & - & -- & $\mathbf{U}$ & $07-13-90$ \\
\hline 20N19W04DCBA01 & 3,425 & -. & Qg & 525 & -- & - & -- & $\mathbf{U}$ & $07-30-91$ \\
\hline $19 \mathrm{~N} 2$ I W 17BCBB 01 & 2,640 & -- & Qal & - & -. & - & -- & $\mathbf{U}$ & $08-01-91$ \\
\hline 19N20W06AAA 01 & 2,920 & --74 & Qg & 18 & 30.0 & - & -- & I & $07-30-91$ \\
\hline 19N20W35AAA 01 & 2,805 & $-\quad-67$ & $\mathrm{Qg}$ & 54 & 6.0 & o & -- & $\mathbf{H}$ & $07-30-91$ \\
\hline 19N19W20BBAA01 & 2,883 & - & Qg & 90 & 6.0 & $\cdot$ & - & $\mathrm{U}$ & 07-30-91 \\
\hline 18N21W05ADCB01 & 2,550 & 09-26-84 & Qal & 320 & 6.0 & $\mathbf{P}$ & 175 & $\mathbf{U}$ & $08-01-91$ \\
\hline $18 \mathrm{~N} 21 \mathrm{~W} 21 \mathrm{BCBB} 01$ & 2,580 & $08-30-84$ & Qal & 160 & 6.0 & $\mathbf{P}$ & 110 & $\mathbf{U}$ & $08-02-91$ \\
\hline 18N20W03BAAB01 & 2,740 & $05-08-79$ & $\mathrm{Qg}$ & 47 & 6.0 & o & -- & $\mathrm{U}$ & 08-01-91 \\
\hline 18N20W14DBDC01 & 2,895 & $--70^{2}$ & $\mathrm{Qg}$ & 30 & 48.0 & - & -- & I & $08-01-91$ \\
\hline 18N20W16ABBB 01 & 2,800 & $04-12-85$ & $\mathrm{Qg}$ & 100 & 6.0 & $\mathbf{s}$ & 95 & $\mathbf{H}$ & $08-06-91$ \\
\hline $18 \mathrm{~N} 19 \mathrm{~W} 10 \mathrm{BCCC} 01$ & 3,300 & $03-01-71$ & $\mathrm{Qg}$ & -- & -- & $\mathrm{O}$ & -- & $\mathrm{U}$ & $08-01-91$ \\
\hline $18 \mathrm{~N} 19 \mathrm{~W} 28 \mathrm{CCDB} 01$ & 3,360 & $08-28-84$ & Qal & 147 & 6.0 & $\mathbf{S}$ & 142 & $\mathbf{U}$ & $07-12-91$ \\
\hline 18N19W30DCBB01 & 3,143 & $04-16-75$ & $\mathrm{Qg}$ & 160 & 6.0 & o & -- & $\mathrm{U}$ & $08-01-91$ \\
\hline \multicolumn{10}{|c|}{ Missoula Valley } \\
\hline $17 \mathrm{~N} 24 \mathrm{~W} 36 \mathrm{DDDA0} 1$ & 3,625 & $06-05-78$ & Qal & 38 & 6.0 & $\mathbf{P}$ & 18 & $\mathbf{H}$ & $07-13-91$ \\
\hline 16N23W17DDAA01 & 3,390 & $07-17-90$ & Qal & 39 & 6.0 & $\mathbf{P}$ & 33 & $\mathbf{H}$ & 07-13-91 \\
\hline $16 \mathrm{~N} 23 \mathrm{~W} 21 \mathrm{BCBC} 01$ & 3,420 & $09-07-83$ & Yms & 250 & 6.0 & $\mathbf{P}$ & 100 & $\mathbf{H}$ & $07-14-91$ \\
\hline 16N23W27BBCC01 & 3,270 & $10-29-87$ & Qal & 40 & 6.0 & $\mathbf{O}$ & -- & $\mathbf{H}$ & 07-13-91 \\
\hline 15N23W0IADAA01 & 3,300 & $06-06-78$ & Ts & 100 & 6.0 & $\mathbf{P}$ & 25 & $\mathbf{H}$ & $07-15-91$ \\
\hline 15N23W01BDCB01 & 3,145 & $07-13-81$ & Qal & 66 & 6.0 & $\mathbf{P}$ & 48 & $\mathbf{H}$ & $07-14-91$ \\
\hline $15 \mathrm{~N} 23 \mathrm{~W} 12 \mathrm{ABCA0} 1$ & 3,120 & $07-20-83$ & $\mathrm{Qg}$ & 61 & 6.0 & $\mathbf{P}$ & 45 & $\mathbf{H}$ & $07-15-91$ \\
\hline $15 \mathrm{~N} 23 \mathrm{~W} 12 \mathrm{ABDD} 01$ & 3,100 & $12-22-67$ & $\mathrm{Qg}$ & 61 & 5.0 & o & -- & $\mathbf{H}$ & $08-02-91$ \\
\hline 15N23W12ACCA01 & 3,215 & $-\quad-79$ & Yms & 130 & 6.0 & $P$ & 45 & $\mathbf{H}$ & $07-15-91$ \\
\hline 15N22W07DCAB01 & 3,075 & $05-17-84$ & Ts & 80 & 6.0 & $\mathbf{P}$ & 53 & $\mathbf{H}$ & $07-15-91$ \\
\hline
\end{tabular}

21870 
Table 1. Records of selected wells by basin, Northern Rocky Mountains intermontane basins, Montana (Continued)

\begin{tabular}{|c|c|c|c|c|c|c|c|c|c|}
\hline $\begin{array}{c}\text { Water level } \\
\text { (fi) }\end{array}$ & $\begin{array}{c}\text { Dis- } \\
\text { charge } \\
\text { (gail/ } \\
\text { min) }\end{array}$ & $\begin{array}{c}\text { Specific } \\
\text { capacity } \\
{[(g a l /} \\
\text { min)/ft] }\end{array}$ & $\begin{array}{l}\text { Source } \\
\text { of dis- } \\
\text { charge } \\
\text { data }\end{array}$ & $\begin{array}{l}\text { Type } \\
\text { of log } \\
\text { avall- } \\
\text { able }\end{array}$ & $\begin{array}{c}\text { Date } \\
\text { water- } \\
\text { quallty } \\
\text { parameter } \\
\text { measured }\end{array}$ & $\begin{array}{c}\text { Speclfic } \\
\text { conduc- } \\
\text { tance } \\
(\mu \mathrm{S} / \mathrm{cm})\end{array}$ & $\begin{array}{c}\text { pH } \\
\text { (stan- } \\
\text { dard } \\
\text { units) }\end{array}$ & $\begin{array}{l}\text { Tem- } \\
\text { per- } \\
\text { ature } \\
\left({ }^{\circ} \mathrm{C}\right)\end{array}$ & Well number \\
\hline \multicolumn{10}{|c|}{ Mission Valley--Continued } \\
\hline 3.18 & -- & -- & - & - & $08-07-75$ & 605 & - & 11.0 & 22N22W04ABA 01 \\
\hline 18.76 & 75 & 81 & $\mathbf{S}$ & D & 08-09-85 & 465 & 7.3 & 9.5 & $22 \mathrm{~N} 22 \mathrm{~W} 26 \mathrm{DDDD} 01$ \\
\hline 8.10 & -- & -- & - & - & -- & -- & -- & -- & 22N21W28DDDC01 \\
\hline 169.32 & -- & -- & - & - & -- & - & -- & -- & 22N21W28DDDD01 \\
\hline 202.23 & -- & -- & - & - & -- & - & -- & -- & 22N20W23DAD 01 \\
\hline 39.36 & -- & -- & - & D & -- & -- & -. & -- & $21 \mathrm{~N} 22 \mathrm{~W} 36 \mathrm{BDCC} 01$ \\
\hline 37.55 & 25 & 6.4 & $\mathbf{S}$ & D & -- & -- & -- & -- & $21 \mathrm{~N} 22 \mathrm{~W} 36 \mathrm{BDCC0}$ \\
\hline 175.85 & -- & - & - & - & -- & -- & -- & -. & $21 \mathrm{~N} 21 \mathrm{~W} 35 \mathrm{CCC} 01$ \\
\hline 71.28 & .. & -- & - & - & $07-29-69$ & 380 & - & 16.0 & $21 \mathrm{~N} 21 \mathrm{~W} 36 \mathrm{CBA} \quad 01$ \\
\hline 7.86 & 290 & -. & $\mathbf{s}$ & - & $07-18-74$ & 400 & -- & 14.5 & $21 \mathrm{~N} 20 \mathrm{~W} 14 \mathrm{ACB} \quad 01$ \\
\hline 49.39 & -- & -- & - & - & $08-26-74$ & 260 & -- & 12.0 & $21 \mathrm{~N} 20 \mathrm{~W} 24 \mathrm{CAAA0} 2$ \\
\hline 59.53 & -- & -- & - & - & -- & -- & -- & - & $21 \mathrm{~N} 20 \mathrm{~W} 31 \mathrm{AABB} 01$ \\
\hline 157.74 & -- & -- & - & - & -- & -- & -- & -- & 20N20W26CCBD01 \\
\hline 75.44 & -- & -- & - & - & -- & -- & -- & -- & 20N19W04DCBA01 \\
\hline 49.91 & -- & .- & - & - & -- & -- & -- & $\ldots$ & $19 \mathrm{~N} 21 \mathrm{~W} 17 \mathrm{BCBB} 01$ \\
\hline 7.62 & 600 & -- & - & - & $09-10-75$ & 220 & 7.5 & 12.5 & 19N20W06AAA 01 \\
\hline 43.41 & $-\cdot$ & -- & - & - & -- & -- & -- & -- & 19N20W35AAA 01 \\
\hline 42.67 & -- & -- & - & - & -- & -- & -- & -- & 19N19W20BBAA01 \\
\hline+7.23 & 65 & -- & $\mathbf{S}$ & D & $10-13-84$ & 860 & 7.9 & 13.0 & 18N21W05ADCB01 \\
\hline+11.55 & -- & -- & - & D & $10-13-84$ & 385 & 8.6 & 10.0 & 18N21W21BCBB01 \\
\hline 19.66 & 25 & 8.3 & D & D & -- & .. & -- & .. & 18N20W03BAAB01 \\
\hline 14.15 & 500 & -- & $\mathbf{R}$ & - & $06-03-83$ & 246 & 7.4 & 9.5 & 18N20W14DBDC01 \\
\hline 57.48 & -- & -- & - & D & -- & -- & -- & -- & $18 \mathrm{~N} 20 \mathrm{~W} 16 \mathrm{ABBB} 01$ \\
\hline 291.30 & 5 & 1.7 & D & D & -- & -- & -- & -. & $18 \mathrm{~N} 19 \mathrm{~W} 10 \mathrm{BCCC} 01$ \\
\hline 101.70 & 40 & 26 & $\mathbf{S}$ & D & $10-14-84$ & 182 & 8.8 & 8.0 & $18 \mathrm{~N} 19 \mathrm{~W} 28 \mathrm{CCDB0} 1$ \\
\hline 18.92 & -- & -- & - & D & -- & - & -- & - & 18N19W30DCBB01 \\
\hline \multicolumn{10}{|c|}{ Missoula Valley } \\
\hline 15.76 & 12 & 1.5 & D & D & $07-13-91$ & 158 & 6.7 & 6.5 & 17N24W36DDDA01 \\
\hline 18.54 & 5 & .3 & D & D & -- & -. & -- & -- & 16N23W17DDAA01 \\
\hline 29.45 & 5 & .4 & D & D & $07-14-91$ & 219 & 6.8 & 8.5 & $16 \mathrm{~N} 23 \mathrm{~W} 21 \mathrm{BCBC} 01$ \\
\hline 10.05 & 20 & 2.0 & D & D & 07-13-91 & 99 & 6.5 & 6.5 & $16 \mathrm{~N} 23 \mathrm{~W} 27 \mathrm{BBCC01}$ \\
\hline 45.99 & -- & -- & $\cdot$ & $\mathbf{D}$ & -- & -- & - & -- & 15N23W01 ADAA01 \\
\hline 39.90 & 20 & 2.9 & D & D & $07-14-91$ & 86 & 5.7 & 9.0 & $15 \mathrm{~N} 23 \mathrm{~W} 01 \mathrm{BDCB} 01$ \\
\hline 49.02 & 10 & 2.0 & D & D & $07-15-91$ & 101 & 6.5 & 8.5 & $15 \mathrm{~N} 23 \mathrm{~W} 12 \mathrm{ABCA0} 1$ \\
\hline 34.75 & 25 & 6.3 & D & D & -- & -- & -- & - & 15N23W12ABDD01 \\
\hline 29.60 & 10 & .1 & D & $\mathbf{D}$ & -- & -- & - & -- & $15 \mathrm{~N} 23 \mathrm{~W} 12 \mathrm{ACCA01}$ \\
\hline 25.19 & 12 & 1.1 & D & D & $07-15-91$ & 515 & 7.0 & 12.0 & 15N22W07DCAB01 \\
\hline
\end{tabular}


Table 1. Records of selected wells by basin, Northern Rocky Mountains intermontane basins, Montana (Continued)

\begin{tabular}{|c|c|c|c|c|c|c|c|c|c|}
\hline Woll number & $\begin{array}{c}\text { Aititude } \\
\text { of land } \\
\text { surface } \\
\text { (ft) }\end{array}$ & $\begin{array}{l}\text { Date well } \\
\text { constructed }\end{array}$ & $\begin{array}{c}\text { Geologic } \\
\text { unit }\end{array}$ & $\begin{array}{l}\text { Depth } \\
\text { of } \\
\text { well } \\
\text { (ft) }\end{array}$ & $\begin{array}{c}\text { Dlam- } \\
\text { oler } \\
\text { of } \\
\text { caeing } \\
\text { (In.) }\end{array}$ & $\begin{array}{l}\text { Type } \\
\text { of } \\
\text { finlah }\end{array}$ & $\begin{array}{c}\text { Top of } \\
\text { open } \\
\text { Inter- } \\
\text { val } \\
\text { (ft) }\end{array}$ & $\begin{array}{c}\text { Primary } \\
\text { use of } \\
\text { water }\end{array}$ & $\begin{array}{l}\text { Date water } \\
\text { level } \\
\text { measured }\end{array}$ \\
\hline \multicolumn{10}{|c|}{ Missoula Valley-Continued } \\
\hline $15 \mathrm{~N} 22 \mathrm{~W} 11 \mathrm{CACC} 1$ & 3,380 & $03-07-88$ & Ts & 140 & 6.0 & $\mathbf{P}$ & 80 & $\mathbf{H}$ & 07-16-91 \\
\hline 15N22W 14DCBA01 & 3,140 & $03-21-89$ & QTsu & 119 & 6.0 & $\mathbf{P}$ & 90 & $\mathbf{H}$ & $07-16-91$ \\
\hline $15 \mathrm{~N} 22 \mathrm{~W} 14 \mathrm{DCBB} 01$ & 3,140 & $03-24-70$ & $\mathrm{Qg}$ & 80 & 6.0 & o & - & $\mathbf{H}$ & $07-16-91$ \\
\hline 15N22W25ADBC01 & 3,020 & $12-18-72$ & Qal & 115 & 6.0 & o & -- & $\mathbf{H}$ & $07-25-91$ \\
\hline $15 \mathrm{~N} 22 \mathrm{~W} 26 \mathrm{AACB} 01$ & 3,012 & $06-23-60$ & Qal & 121 & 10.0 & $\mathbf{P}$ & 113 & $\mathbf{I}$ & $10-04-62$ \\
\hline 15N22W28BADC01 & 2,999 & $05-20-67$ & Qg & 138 & 6.0 & - & -- & $\mathbf{H}$ & $07-15-91$ \\
\hline $15 N 22 W 33 B A C 01$ & 3,000 & -- & Qal & 150 & -- & - & -- & $\mathbf{N}$ & $07-15-91$ \\
\hline $15 \mathrm{~N} 22 \mathrm{~W} 33 \mathrm{BAC} 02$ & 3,000 & 02-08-79 & Qal & 80 & 8.0 & o & -- & 1 & $07-15-91$ \\
\hline 15N22W33BDDD01 & 3,085 & $06-26-79$ & QTsu & 260 & 6.0 & $\mathbf{P}$ & 120 & H & $07-17-91$ \\
\hline $15 \mathrm{~N} 22 \mathrm{~W} 33 \mathrm{DBCC} 01$ & 3,078 & $06-21-79$ & Ts & 420 & 6.0 & $\mathbf{P}$ & 280 & $\mathbf{I}$ & $07-15-91$ \\
\hline 15N21W17CCCC01 & 3,429 & 07-16-79 & Ts & 2,401 & 6.0 & $\mathbf{P}$ & 87 & $\mathbf{U}$ & 07-23-91 \\
\hline 15N21W17DBCD01 & 3,520 & 09-19-73 & QTsu & 71 & 6.0 & o & -- & $\mathbf{H}$ & $07-23-91$ \\
\hline 15N21W20DDDC01 & 3,195 & $11-30-84$ & Ts & 320 & 6.0 & $\mathbf{P}$ & 230 & $\mathbf{S}$ & $07-16-91$ \\
\hline 15N21W25BBBA01 & 3,515 & $03-11-88$ & Yms & 500 & 6.0 & 0 & - & $\mathbf{H}$ & $07-24-91$ \\
\hline $15 \mathrm{~N} 21 \mathrm{~W} 25 \mathrm{CBBC} 01$ & 3,350 & $06-12-86$ & Ts & 260 & 6.0 & $\mathbf{P}$ & 105 & $\mathbf{H}$ & $07-23-91$ \\
\hline $15 \mathrm{~N} 21 \mathrm{~W} 25 \mathrm{CCBA0} 01$ & 3,245 & 07-01-82 & Ts & 180 & 6.0 & $\mathbf{P}$ & 100 & $\mathbf{H}$ & $07-24-91$ \\
\hline 15N21W28DCCB01 & 3,030 & $03-30-73$ & Qal & 183 & 6.0 & $\mathbf{P}$ & 179 & $\mathbf{H}$ & $07-16-91$ \\
\hline $15 \mathrm{~N} 21 \mathrm{~W} 29 \mathrm{ADBC0}$ & 3,020 & $05-23-91$ & Qal & 186 & 8.0 & 0 & - & $\mathbf{H}$ & $07-16-91$ \\
\hline 15N21W30BBAC01 & 3,020 & 05-05-80 & Qal & 66 & 6.0 & $\mathbf{P}$ & 40 & $\mathbf{H}$ & $07-17-91$ \\
\hline 15N21W30DDBD01 & 3,023 & $04-18-89$ & Qal & 188 & 6.0 & $\mathrm{O}$ & - & $\mathbf{H}$ & $07-17-91$ \\
\hline 14N22W08BBDA01 & 3,050 & $06-23-88$ & Ts & 141 & 6.0 & o & -- & $\mathbf{H}$ & $07-14-91$ \\
\hline 14N22W08BDDA01 & 3,035 & $08-16-84$ & Ts & 190 & 6.0 & $\mathrm{O}$ & -- & $\mathbf{H}$ & $07-14-91$ \\
\hline 14N21W01DDBB01 & 3,180 & $-\quad-89$ & Ts & 60 & 6.0 & 0 & -- & $\mathrm{U}$ & $07-12-91$ \\
\hline 14N2IW11ABAB01 & 3,058 & - & Qal & 24 & 2.0 & - & -- & $\mathrm{U}$ & $07-10-91$ \\
\hline $14 \mathrm{~N} 21 \mathrm{~W} 13 \mathrm{BBAA} 01$ & 3,055 & $06-24-76$ & Qal & 163 & 6.0 & 0 & -- & $\mathbf{H}$ & $07-11-91$ \\
\hline 14N21 W13CACC01 & 3,065 & $06-28-74$ & Qal & 176 & 6.0 & $\mathbf{P}$ & 166 & $\mathbf{N}$ & $07-10-91$ \\
\hline 14N21W13DCDA01 & 3,068 & $08-07-87$ & Qal & 169 & 18.0 & $\mathbf{s}$ & 144 & $\mathbf{N}$ & $08-07-87$ \\
\hline 14N21 W13DDCA01 & 3,068 & -- & Qal & 190 & - & - & -- & $\mathbf{U}$ & $07-11-91$ \\
\hline 14N21W13DDCC01 & 3,068 & $04-20-87$ & Qal & 193 & 18.0 & $\mathbf{s}$ & 150 & $\mathbf{N}$ & $04-20-87$ \\
\hline 14N21W24BAAC01 & 3,062 & $04-10-57$ & Qal & 174 & 16.0 & $\mathbf{P}$ & 147 & $\mathbf{N}$ & $04-10-57$ \\
\hline 14N21 W24BAAC02 & 3,062 & $05-21-57$ & Qal & 174 & 16.0 & $\mathbf{P}$ & 148 & $\mathbf{N}$ & $05-21-57$ \\
\hline 14N21W24DBA 01 & 3,062 & $-\quad-58$ & Qal & 33 & 36.0 & 0 & -- & $\mathbf{U}$ & $07-14-81$ \\
\hline 14N21 W25ABCC01 & 3,055 & --71 & Qal & 23 & 6.0 & - & -- & $\mathbf{U}$ & $07-10-91$ \\
\hline $14 \mathrm{~N} 21 \mathrm{~W} 25 \mathrm{BCAA01}$ & 3,051 & -- & Qal & 115 & 4.5 & - & -- & $\mathbf{U}$ & $07-10-91$ \\
\hline 14N21W25BCAC01 & 3,050 & $12-05-66$ & Qal & 51 & 6.0 & - & - & $\mathbf{U}$ & $07-10-91$ \\
\hline 14N21W25CADD01 & 3,055 & -- & Qal & 30 & 6.0 & - & -- & $\mathrm{U}$ & $08-25-82$ \\
\hline 14N2IW25CADD02 & 3,055 & -- & Qal & 125 & 6.0 & - & -- & $\mathrm{u}$ & $07-10-91$ \\
\hline 14N21W25CCDA01 & 3,053 & $03-23-66$ & Qal & 129 & 6.0 & $\mathbf{S}$ & 128 & $\mathrm{U}$ & $07-10-91$ \\
\hline 14N21W25CCDA02 & 3,053 & $08-18-66$ & Qal & 160 & 18.0 & $\mathbf{S}$ & 113 & $\mathbf{N}$ & $08-18-66$ \\
\hline 14N20W04ADBD01 & 3,380 & $09-14-76$ & Qal & 52 & 6.0 & $\mathbf{P}$ & 45 & $\mathbf{H}$ & $08-02-91$ \\
\hline
\end{tabular}


Table 1. Records of selected wells by basin, Northern Rocky Mountains intermontane basins, Montana (Continued)

\begin{tabular}{|c|c|c|c|c|c|c|c|c|c|}
\hline $\begin{array}{c}\text { Water level } \\
\text { (ft) }\end{array}$ & $\begin{array}{c}\text { Dis- } \\
\text { charge } \\
\text { (gal/ } \\
\text { min) }\end{array}$ & $\begin{array}{c}\text { Specific } \\
\text { capacity } \\
\text { [(gal/ } \\
\text { min)/ft] }\end{array}$ & $\begin{array}{l}\text { Source } \\
\text { of dis- } \\
\text { charge } \\
\text { date }\end{array}$ & $\begin{array}{l}\text { Typs } \\
\text { of log } \\
\text { avali- } \\
\text { able }\end{array}$ & $\begin{array}{c}\text { Date } \\
\text { wator- } \\
\text { quality } \\
\text { parameter } \\
\text { measured }\end{array}$ & $\begin{array}{c}\text { Speclfic } \\
\text { concuc- } \\
\text { tence } \\
(\mu \mathrm{S} / \mathrm{cm})\end{array}$ & $\begin{array}{c}\text { pH } \\
\text { (sten- } \\
\text { dard } \\
\text { unlte) }\end{array}$ & $\begin{array}{l}\text { Tem- } \\
\text { per- } \\
\text { ature } \\
\left(^{\circ} \mathrm{C}\right)\end{array}$ & Well number \\
\hline
\end{tabular}

\begin{tabular}{|c|c|c|c|c|c|c|c|c|c|}
\hline \multicolumn{10}{|c|}{ Missoula Valley-Continued } \\
\hline 51.90 & -- & $\cdots$ & - & D & $07-|6-9|$ & 993 & 7.8 & 10.5 & 15N22W $11 C A C C 01$ \\
\hline 59.16 & 35 & 2.5 & D & D & $07-16-91$ & 287 & 7.0 & 8.5 & $15 \mathrm{~N} 22 \mathrm{~W} 14 \mathrm{DCBA01}$ \\
\hline 56.91 & 9 & 6.1 & $\mathbf{S}$ & D & $07-16-91$ & 279 & 7.0 & 8.5 & $15 \mathrm{~N} 22 \mathrm{~W} 14 \mathrm{DCBB} 01$ \\
\hline 16.50 & 75 & 9.4 & D & D & $07-25-91$ & 324 & 7.5 & 12.0 & $15 \mathrm{~N} 22 \mathrm{~W} 25 \mathrm{ADBC} 01$ \\
\hline 3.10 & 300 & 5.5 & $\mathbf{D}$ & D & $07-17-91$ & 287 & 7.6 & 11.5 & $15 \mathrm{~N} 22 \mathrm{~W} 26 \mathrm{AACB} 01$ \\
\hline 4.79 & 15 & .6 & D & D & $07-15-91$ & 269 & 6.9 & 9.5 & 15N22W 28BADC01 \\
\hline 42.74 & -- & -- & - & - & -- & -- & - & - & 15N22W33BAC 01 \\
\hline 41.48 & 90 & 9.0 & $\mathrm{D}$ & $\mathrm{D}$ & -- & -- & -- & - & $15 \mathrm{~N} 22 \mathrm{~W} 33 \mathrm{BAC} 02$ \\
\hline 120.10 & 30 & -- & $\mathrm{D}$ & $\mathbf{D}$ & $07-15-91$ & 750 & 7.7 & 12.0 & $15 \mathrm{~N} 22 \mathrm{~W} 33 \mathrm{BDDD} 01$ \\
\hline 91.68 & 20 & -- & $\mathrm{D}$ & D & $07-15-91$ & 456 & 6.8 & 10.0 & $15 \mathrm{~N} 22 \mathrm{~W} 33 \mathrm{DBCC} 01$ \\
\hline 165.03 & 5 & -- & D & D & -- & -- & -- & - & $15 \mathrm{~N} 21 \mathrm{~W} 17 \mathrm{CCCCO}$ \\
\hline 57.19 & 20 & 4.0 & $\mathbf{D}$ & D & $07-23-91$ & 426 & 7.7 & 10.0 & 15N21 W17DBCD01 \\
\hline 176.50 & 1 & .1 & $\mathbf{D}$ & $\mathbf{D}$ & -. & -- & -- & -. & 15N21 W20DDDC01 \\
\hline 14.57 & 7 & -- & $\mathrm{D}$ & D & $07-24-91$ & 351 & 7.6 & 12.0 & 15N21 W25BBBA01 \\
\hline 125.28 & 2 & .1 & D & $\mathbf{D}$ & -- & -- & -- & $\cdots$ & $15 \mathrm{~N} 21 \mathrm{~W} 25 \mathrm{CBBC} 01$ \\
\hline 65.36 & 6 & .5 & D & D & $07-24-91$ & 258 & 7.8 & 10.0 & 15N21W25CCBA01 \\
\hline 10.84 & 50 & 60 & D & D & -- & - & -- & -- & 15N21W28DCCB01 \\
\hline 7.48 & 50 & 1.0 & D & D & -- & -- & -- & -- & $15 \mathrm{~N} 21 \mathrm{~W} 29 \mathrm{ADBC} 01$ \\
\hline 15.80 & 12 & 14 & $\mathbf{S}$ & D & $07-17-91$ & 300 & 7.0 & 11.5 & 15N21W30BBAC01 \\
\hline 11.16 & 9 & 4.2 & $\mathbf{S}$ & $\mathrm{D}$ & $07-17-91$ & 307 & 8.0 & 12.0 & $15 \mathrm{~N} 21 \mathrm{~W} 30 \mathrm{DDBD} 01$ \\
\hline 108.45 & 30 & 3.0 & D & $\mathbf{D}$ & $07-14-91$ & 302 & 7.1 & 10.0 & 14N22W08BBDA01 \\
\hline 55.48 & 20 & 5.0 & D & D & $07-14-91$ & 318 & 7.0 & 10.5 & 14N22W08BDDA01 \\
\hline 32.80 & -- & -- & - & - & -- & -- & -- & -- & 14N21W01DDBB01 \\
\hline 16.13 & -- & -- & - & - & -- & -- & -- &.- & $14 \mathrm{~N} 21 \mathrm{~W} 11 \mathrm{ABAB} 01$ \\
\hline 15.97 & 99 & 2.4 & $\mathbf{D}$ & $\mathbf{D}$ & -- & -- & -- & -- & 14N21W13BBAA01 \\
\hline 24.58 & 150 & -- & D & D & -- & -- & -- & -- & $14 \mathrm{~N} 21 \mathrm{~W} 13 \mathrm{CACC} 1$ \\
\hline 27,67 & 2,000 & 66 & D & $\mathbf{D}$ & -- & -- & -- & -- & 14N21 W13DCDA01 \\
\hline 33,22 & -- & -- & - & - & -- & -- & -- & -- & 14N21 W13DDCA01 \\
\hline 27.08 & 2,300 & 61 & $\mathrm{D}$ & $\mathrm{D}$ & $07-11-91$ & 388 & 7.7 & 10.0 & 14N21W13DDCC01 \\
\hline 22.83 & 1,500 & 51 & D & $\mathrm{D}$ & $07-10-91$ & 1,130 & 7.6 & 16.0 & 14N21W24BAAC0I \\
\hline *22 & 1,500 & 58 & D & $\mathrm{D}$ & -- & - & -- & -- & 14N21W24BAAC02 \\
\hline 19.81 & -- & -- & - & - & - & - & -- & -- & $14 \mathrm{~N} 21 \mathrm{~W} 24 \mathrm{DBA} \quad 01$ \\
\hline 12.10 & -- & -- & - & - & -- & -- & $\cdots$ & -- & $14 \mathrm{~N} 21 \mathrm{~W} 25 \mathrm{ABCC} 01$ \\
\hline 15.78 & -. & -- & - & - & -. & - & -- & - & 14N21W25BCAA01 \\
\hline 10.95 & -- & $\cdots$ & - & $\cdot$ & -- & -- & - & - & $14 \mathrm{~N} 21 \mathrm{~W} 25 \mathrm{BCAC0} 1$ \\
\hline 10.87 & -- & -- & - & - & -- & - & -- & -- & 14N21 W25CADDO 1 \\
\hline 10.34 & -- & - & - & - & -- & -- & - & -- & 14N21W25CADD02 \\
\hline 21.79 & 23 & 1.2 & D & D & $-\cdot$ & -. & -. & -- & 14N21W25CCDA01 \\
\hline 8.40 & 2,000 & 95 & $\mathrm{D}$ & $\mathbf{D}$ & $07-10-91$ & 353 & 7.3 & 11.0 & $14 \mathrm{~N} 21 \mathrm{~W} 25 \mathrm{CCDA02}$ \\
\hline 7.95 & 40 & 1.3 & D & $\mathrm{D}$ & -- & -- & -- & -- & 14N20W04ADBD01 \\
\hline
\end{tabular}


Table 1. Records of selected wells by basin, Northern Rocky Mountains intermontane basins, Montana (Continued)

\begin{tabular}{|c|c|c|c|c|c|c|c|c|c|}
\hline Well number & $\begin{array}{l}\text { Altitude } \\
\text { of land } \\
\text { surface } \\
\text { (ft) }\end{array}$ & $\begin{array}{c}\text { Dato well } \\
\text { constructed }\end{array}$ & $\begin{array}{c}\text { Geologic } \\
\text { unit }\end{array}$ & $\begin{array}{l}\text { Depth } \\
\text { of well } \\
\text { (ft) }\end{array}$ & $\begin{array}{c}\text { Dlam- } \\
\text { oter } \\
\text { of } \\
\text { casing } \\
\text { (In.) }\end{array}$ & $\begin{array}{l}\text { Type } \\
\text { of } \\
\text { finish }\end{array}$ & $\begin{array}{l}\text { Top of } \\
\text { open } \\
\text { Inter- } \\
\text { val } \\
\text { (ft) }\end{array}$ & $\begin{array}{l}\text { Primary } \\
\text { uee of } \\
\text { water }\end{array}$ & $\begin{array}{c}\text { Date water } \\
\text { level } \\
\text { measured }\end{array}$ \\
\hline \multicolumn{10}{|c|}{ Missoula Valley-Continued } \\
\hline 14N20W08DDCC01 & 3,380 & $09-17.85$ & Ts & 80 & 6.0 & $\mathbf{P}$ & 45 & $\mathbf{H}$ & $07-12-91$ \\
\hline 14N20W24ADBC01 & 3,520 & $01-16-91$ & Ts & 140 & 6.0 & $\mathbf{P}$ & 60 & $\mathbf{U}$ & $07-24-91$ \\
\hline 14N20W24ADBD01 & 3,540 & $09-24-79$ & Ts & 2,907 & 6.0 & $\mathbf{P}$ & 645 & $\mathbf{I}$ & $07-26-91$ \\
\hline 14N20W24ADBD02 & 3,540 & $06-01-71$ & Qal & 85 & 6.0 & 0 & - & $\mathbf{H}$ & $07-25-91$ \\
\hline 14N20W24ADCB01 & 3,520 & $01-15-91$ & Ts & 120 & 6.0 & $\mathbf{P}$ & 50 & $\mathbf{U}$ & $07-24-91$ \\
\hline 14N20W28DBDD01 & 3,199 & $12-10-74$ & Ts & 372 & 6.0 & $\mathbf{P}$ & 120 & $\mathbf{N}$ & $07-13-91$ \\
\hline 14N20W28DCCC01 & 3,182 & $05-13-76$ & Qg & 77 & 6.0 & $\mathrm{O}$ & -- & $\mathbf{H}$ & $07-11-91$ \\
\hline 14N20W29DCCA01 & 3,196 & $04-22-81$ & Yms & 185 & 6.0 & $\mathbf{P}$ & 177 & $\mathbf{H}$ & $07-12-91$ \\
\hline 14N20W32DCCA01 & 3,076 & $09-13-78$ & Qal & 124 & 6.0 & o & - & $\mathbf{H}$ & $07-13-91$ \\
\hline 14N20W35BACB01 & 3,230 & $10-30-68$ & QTsu & 344 & 16.0 & $\mathbf{P}$ & 177 & $\mathbf{P}$ & $07-12-91$ \\
\hline 14N19W15BABD01 & 4,000 & $09 .-84$ & Yms & 100 & 6.0 & $\mathrm{x}$ & 31 & $\mathbf{H}$ & $08-01-91$ \\
\hline 14N19W36BBCC01 & 3,665 & $01-25-79$ & Qal & 100 & 6.0 & $\mathbf{P}$ & 60 & $\mathbf{H}$ & $08-02-91$ \\
\hline 13N21W01DCAD01 & 3,250 & $09-06-78$ & Yms & 427 & 6.0 & $\mathbf{P}$ & 197 & $\mathbf{H}$ & 07-13-91 \\
\hline 13N21W11 AADC01 & 3,960 & $06-26-76$ & Yms & 400 & 6.6 & $\mathbf{P}$ & 290 & $\mathbf{H}$ & $06-26-76$ \\
\hline 13 N20W08CBB 01 & 3,082 & -. & Qal & 100 & -- & - & - & $\mathbf{H}$ & $07-11-91$ \\
\hline |3N19W12BBBB01 & 3,640 & $05-19-66$ & Yms & 1,145 & 6.0 & $\mathrm{X}$ & 333 & $\mathbf{H}$ & $08-02-91$ \\
\hline 13N19W14BAAA01 & 3,380 & 07-08-64 & Qal & 127 & 12.0 & $\mathbf{P}$ & 70 & $\mathbf{U}$ & $07-12-91$ \\
\hline 13N19W16CBBC01 & 3,187 & $07-30-69$ & Qal & 110 & 16.0 & $\mathbf{P}$ & 85 & $\mathbf{P}$ & $07-12-91$ \\
\hline 13N19W24ABCA01 & 3,260 & $04-27-64$ & Qal & 108 & 12.0 & $\mathbf{P}$ & 80 & $\mathbf{P}$ & $07-12-91$ \\
\hline 13N19W27ACBC01 & 3,218 & $10-24-90$ & Qal & 200 & 8.0 & $\mathbf{P}$ & 110 & $\mathbf{U}$ & $07-26-91$ \\
\hline 13N19W27CCAD01 & 3,210 & $06-17-69$ & Qal & 137 & 16.0 & $\mathbf{P}$ & 90 & $\mathbf{P}$ & $07-12-91$ \\
\hline 13N19W30ADBA01 & 3,165 & $07-10-72$ & Qal & 125 & 16.0 & $\mathbf{P}$ & 92 & $\mathbf{P}$ & $07-12-91$ \\
\hline \multicolumn{10}{|c|}{ Plains Valley } \\
\hline $21 \mathrm{~N} 26 \mathrm{~W} 25 \mathrm{DCDCO} 1$ & 2,970 & $10-11-84$ & Yms & 510 & 6.0 & $\mathbf{P}$ & 380 & $\mathbf{H}$ & 09-06-91 \\
\hline 20N27W01CBCD01 & 2,475 & $04-17-71$ & Qg & 97 & 6.0 & - & - & $\mathbf{H}$ & $09-05-91$ \\
\hline 20N27W03ABDB01 & 2,448 & $09-11-73$ & Yms & 120 & 6.0 & $\mathrm{X}$ & 95 & $\mathbf{H}$ & $09-04-91$ \\
\hline 20N27W10AAAB0I & 2,600 & $12-14-78$ & Yms & 250 & 6.0 & $\mathrm{X}$ & 25 & $\mathbf{H}$ & $09-04-91$ \\
\hline $20 \mathrm{~N} 27 \mathrm{~W} 10 \mathrm{ABAD} 01$ & 2,450 & - & Yms & 80 & -- & - & -- & $\mathbf{H}$ & $09-04-91$ \\
\hline 20N27W 13DDCD01 & 2,605 & $07-22-89$ & $\mathrm{Qg}$ & 203 & 6.0 & $\mathrm{O}$ & -- & $\mathbf{H}$ & $09-04-91$ \\
\hline $20 \mathrm{~N} 27 \mathrm{~W} 24 \mathrm{BDBC} 01$ & 2,645 & $05-22-86$ & $\mathrm{Qg}$ & 31 & 6.0 & $\mathbf{O}$ & -- & $\mathbf{H}$ & $09-04-91$ \\
\hline 20N26W03DCDA01 & 2,605 & $10-17-89$ & Yms & 320 & 6.0 & $\mathbf{P}$ & 134 & $\mathbf{H}$ & $09-06-91$ \\
\hline 20N26W08BACD01 & 2,660 & $08-\quad-80$ & Qg & 250 & 6.0 & $\mathbf{P}$ & 80 & H & 09-04-91 \\
\hline $20 \mathrm{~N} 26 \mathrm{~W} 09 \mathrm{CCBD} 01$ & 2,470 & $06-12-74$ & Qal & 160 & 6.0 & $\mathbf{P}$ & 66 & $\mathbf{H}$ & $09-03-91$ \\
\hline 20N26W14ACCB01 & 2,680 & $06-06-87$ & Yms & 275 & 6.0 & $\mathrm{X}$ & 259 & H & $09-05-91$ \\
\hline 20N26W15ACAB01 & 2,475 & $04-\quad-68$ & Qal & 40 & 12.0 & $\mathbf{P}$ & 30 & $\mathbf{I}$ & $09-05-91$ \\
\hline 20N26W15BAAD01 & 2,470 & 06-07-82 & Qal & 189 & 8.0 & $\mathrm{o}$ & - & $\mathbf{I}$ & $09-05-91$ \\
\hline 20N26W 16BADA0I & 2,460 & $04--67$ & Qal & 97 & 12.0 & $\mathbf{P}$ & 76 & I & $09-03-91$ \\
\hline 20N26W23CCBA01 & 2,468 & $09--69$ & Qal & 66 & 12.0 & $\mathbf{P}$ & 14 & 1 & $09-06-91$ \\
\hline
\end{tabular}


Table 1. Records of selected wells by basin, Northern Rocky Mountains intermontane basins, Montana (Continued)

\begin{tabular}{|c|c|c|c|c|c|c|c|c|c|}
\hline $\begin{array}{c}\text { Water levei } \\
\text { (ft) }\end{array}$ & $\begin{array}{c}\text { Dis- } \\
\text { charge } \\
\text { (gal/ } \\
\text { min) }\end{array}$ & $\begin{array}{c}\text { Specific } \\
\text { capacity } \\
\text { [(gal/ } \\
\text { mln)/ft] }\end{array}$ & $\begin{array}{c}\text { Source } \\
\text { of dis- } \\
\text { charge } \\
\text { data }\end{array}$ & $\begin{array}{l}\text { Type } \\
\text { of log } \\
\text { avail- } \\
\text { able }\end{array}$ & $\begin{array}{l}\text { Date } \\
\text { water- } \\
\text { quality } \\
\text { parameter } \\
\text { measured }\end{array}$ & $\begin{array}{c}\text { Specific } \\
\text { conduc- } \\
\text { tance } \\
(\mu S / \mathrm{cm})\end{array}$ & $\begin{array}{c}\text { pH } \\
\text { (stan- } \\
\text { dard } \\
\text { unita) }\end{array}$ & $\begin{array}{l}\text { Tom- } \\
\text { per- } \\
\text { ature } \\
\left({ }^{\circ} \mathrm{C}\right)\end{array}$ & Well number \\
\hline
\end{tabular}

\section{Missoula Valley-Continued}

\begin{tabular}{|c|c|c|c|c|c|c|c|c|c|}
\hline 14.76 & 5 & .4 & D & D & - & -- & -- & -- & 14N20W08DDCC01 \\
\hline 33.68 & 10 & .5 & D & D & -- & -- & -. & -- & 14N20W24ADBC01 \\
\hline 84.55 & 13 & .1 & D & D & $07-26-91$ & 874 & 7.8 & 16.0 & 14N20W24ADBD01 \\
\hline .08 & 8 & .2 & D & D & $07-25-91$ & 188 & 7.1 & 10.0 & 14N20W24ADBD02 \\
\hline 54.19 & 8 & .7 & D & D & -- & -- & -- & -- & 14N20W24ADCB01 \\
\hline *95 & 40 & .2 & $\mathrm{D}$ & D & $07-12-91$ & 212 & 7.3 & 13.0 & 14N20W28DBDD01 \\
\hline 63.81 & 2 & 1.0 & $\mathrm{~S}$ & D & $07-11-91$ & 384 & 7.3 & 11.5 & $14 \mathrm{~N} 20 \mathrm{~W} 28 \mathrm{DCCC} 01$ \\
\hline 116.68 & 15 & 1.3 & D & D & $07-12-91$ & 404 & 6.7 & 12.0 & 14N20W29DCCA01 \\
\hline+.75 & 100 & 11 & D & D & $07-13-91$ & 252 & 7.2 & 10.0 & 14N20W32DCCA01 \\
\hline 111.90 & 500 & 83 & D & D & - & -- & -- & -- & 14N20W35BACB01 \\
\hline 21.07 & 10 & .2 & D & D & -- & -. & - & -. & 14N19W15BABD01 \\
\hline 35.66 & 15 & .6 & $\mathrm{D}$ & D & -- & -- & -- & -- & 14N19W36BBCC01 \\
\hline 190.79 & 10 & .1 & D & D & 07-13-91 & 362 & 7.4 & 15.5 & 13N21 W01DCAD01 \\
\hline$* 260$ & 3 & .2 & $\mathrm{D}$ & D & $07-11-91$ & 425 & 7.1 & 12.0 & 13N21W11AADC01 \\
\hline 11.22 & 6 & 5.6 & $\mathbf{S}$ & - & $07-11-91$ & 300 & 7.3 & 10.5 & $13 \mathrm{~N} 20 \mathrm{~W} 08 \mathrm{CBB} \quad 01$ \\
\hline *282 & 6 &.- & D & D & - & -- & -. & .. & 13N19W12BBBB01 \\
\hline 43.05 & 800 & 13 & D & D & -- & -- & -- & -- & |3N19W|4BAAA01 \\
\hline 45.32 & 1,000 & 290 & D & D & -- & -- & -- & -- & 13N19W16CBBC01 \\
\hline 62.92 & 1,200 & $-\cdot$ & D & D & -- & -- & -- & -- & 13N19W24ABCA01 \\
\hline 73.98 & 350 & 64 & $\mathrm{D}$ & D & -- & -- & -. & -- & $13 \mathrm{~N} 19 \mathrm{~W} 27 \mathrm{ACBC} 01$ \\
\hline 65.07 & 1,000 & 180 & D & D & -- & -- & -- & -- & 13N19W27CCAD01 \\
\hline 33.30 & 1,500 & 1,500 & D & D & -- & -- & -- & -- & 13N19W30ADBA01 \\
\hline \multicolumn{10}{|c|}{ Plains Valley } \\
\hline 90.00 & 6 & $-\cdots$ & D & D & 09-05-91 & 346 & 8.0 & 12.0 & $21 \mathrm{~N} 26 \mathrm{~W} 25 \mathrm{DCDC} 01$ \\
\hline 66.48 & -- & -. & - & D & $09-05-91$ & 139 & 6.9 & 10.5 & 20N27W01CBCD01 \\
\hline 41.14 & 7 & 7.0 & $\mathrm{D}$ & D & $09-04-91$ & 446 & 7.6 & 12.0 & 20N27W03ABDB01 \\
\hline 124.64 & 30 & .4 & D & D & 09-04-91 & 580 & 7.5 & 9.5 & 20N27W10AAABOI \\
\hline 15.86 & 11 & 2.1 & $\mathbf{S}$ & - & $09-04-91$ & 198 & 7.6 & 9.0 & 20N27W10ABAD01 \\
\hline 171.53 & 20 & 2.0 & D & $\mathbf{D}$ & $09-04-91$ & 104 & 7.2 & 9.0 & $20 \mathrm{~N} 27 \mathrm{~W} 13 \mathrm{DDCD} 01$ \\
\hline 16.20 & 20 & 2.5 & D & D & $09-04-91$ & 56 & 7.0 & 10.0 & 20N27W24BDBC01 \\
\hline *207 & 30 & 1.0 & D & D & -- & -- & -- & -- & 20N26W03DCDA01 \\
\hline 101.33 & 30 & .3 & D & D & 09-04-91 & 198 & 6.9 & 13.5 & 20N26W08BACD01 \\
\hline 53.20 & 10 & .3 & D & $\mathrm{D}$ & 09-03-91 & 245 & 7.5 & 11.5 & 20N26W09CCBD01 \\
\hline 94.31 & 10 & .1 & $\mathrm{D}$ & D & $09-05-91$ & 342 & 7.2 & 13.0 & $20 \mathrm{~N} 26 \mathrm{~W} 14 \mathrm{ACCB} 01$ \\
\hline 15.31 & 250 & 17 & $\mathrm{D}$ & D & 09-05-91 & 379 & 6.7 & 12.5 & $20 \mathrm{~N} 26 \mathrm{~W} 15 \mathrm{ACAB} 01$ \\
\hline$* 17$ & 200 & 1.4 & D & D & $09-05-91$ & 168 & 7.0 & 12.5 & 20N26W15BAAD01 \\
\hline 3.77 & - & - & - & D & -- & -- & -. & -- & 20N26W16BADA01 \\
\hline 10.24 & 100 & 2.4 & $\mathrm{D}$ & D & -- & -- & -- & -. & 20N26W23CCBA01 \\
\hline
\end{tabular}


Table 1. Records of selected wells by basin, Northern Rocky Mountains intermontane basins, Montana (Continued)

\begin{tabular}{|c|c|c|c|c|c|c|c|c|c|}
\hline Well number & $\begin{array}{l}\text { Altitude } \\
\text { of lend } \\
\text { surface } \\
\text { (ft) }\end{array}$ & $\begin{array}{l}\text { Date well } \\
\text { constructed }\end{array}$ & $\begin{array}{c}\text { Geologic } \\
\text { unit }\end{array}$ & $\begin{array}{l}\text { Depth } \\
\text { of well } \\
\text { (ft) }\end{array}$ & $\begin{array}{c}\text { Dlam- } \\
\text { eter } \\
\text { of } \\
\text { casing } \\
\text { (In.) }\end{array}$ & $\begin{array}{c}\text { Type } \\
\text { of } \\
\text { finlsh }\end{array}$ & $\begin{array}{l}\text { Top of } \\
\text { open } \\
\text { Inter- } \\
\text { val } \\
\text { (ft) }\end{array}$ & $\begin{array}{l}\text { Primary } \\
\text { use of } \\
\text { water }\end{array}$ & $\begin{array}{c}\text { Date wate } \\
\text { lovel } \\
\text { meacured }\end{array}$ \\
\hline \multicolumn{10}{|c|}{ Plains Valley-Continued } \\
\hline 20N26W24DDC 01 & 2,835 & -- & Qg & 45 & -- & - & -- & $\mathbf{H}$ & 09-05-91 \\
\hline $20 \mathrm{~N} 26 \mathrm{~W} 27 \mathrm{DBBC} 01$ & 2,463 & $08-02-75$ & Qal & 45 & 16.0 & $\mathbf{o}$ & -. & $\mathrm{U}$ & 09-03-91 \\
\hline $20 \mathrm{~N} 26 \mathrm{~W} 27 \mathrm{DDBC} 01$ & 2,465 & $11-10-65$ & Qal & 38 & 48.0 & $\mathbf{P}$ & 28 & $\mathbf{P}$ & 09-03-91 \\
\hline 19N26W01CAB 01 & 2,540 & 08-01-84 & Yms & 235 & 6.0 & $\mathbf{P}$ & 210 & $\mathbf{H}$ & 09-05-91 \\
\hline 19N26W03ACBC01 & 2,580 & $02-05-81$ & Qg & 163 & 6.0 & o & - & $\mathbf{S}$ & 09-04-91 \\
\hline 19N26W03BDAC01 & 2,603 & $03-03-81$ & Yms & 260 & 6.0 & $\mathbf{x}$ & 137 & $\mathbf{H}$ & 09-05-91 \\
\hline \multicolumn{10}{|c|}{ Red Rock Valley } \\
\hline 10S10W29DCCC01 & 5,630 & 04-05-76 & Pzls & 84 & 6.0 & $\mathbf{P}$ & 68 & $\mathbf{H}$ & $10-07-91$ \\
\hline $11 \mathrm{~S} 10 \mathrm{~W} 26 \mathrm{BBCC} 01$ & 5,790 & $01-13-70$ & Ts & 198 & 6.0 & $\mathbf{P}$ & 139 & $\mathbf{P}$ & $10-07-91$ \\
\hline 11S10W27ADAC01 & 5,835 & $10-19-69$ & Ts & 202 & 6.0 & $\mathbf{P}$ & 158 & $\mathbf{P}$ & $10-07-91$ \\
\hline 1IS10W34CCAC01 & 6,120 & $09-12-69$ & Pzls & 410 & 6.0 & $\mathbf{P}$ & 384 & $\mathbf{U}$ & $09-12-69$ \\
\hline 11S08W 12CCBA01 & 6,850 & $10-20-85$ & Ts & 100 & 6.0 & $\mathbf{P}$ & 90 & $\mathbf{H}$ & $10-14-91$ \\
\hline 11S08W22ABBD01 & 6,650 & $06-29-78$ & Ts & 85 & 6.0 & $\mathbf{P}$ & 78 & $\mathbf{H}$ & $10-14-91$ \\
\hline 11S08W32ACAC01 & 6,895 & - & Ts & -- & 5.0 & - & -- & $\mathbf{S}$ & $10-14-91$ \\
\hline 12S10W22CADA0I & 6,365 & $11-06-85$ & Ts & 780 & 10.0 & $\mathbf{P}$ & 645 & $\mathbf{S}$ & $11-06-85$ \\
\hline 12S08W03CBBD01 & 6,670 & $06-29-66$ & Ts & 265 & 6.0 & $\mathbf{p}$ & 250 & $\mathbf{s}$ & $10-14-91$ \\
\hline 12S08W31ABD 01 & 6,565 & $11-09-71$ & Ts & 528 & 6.0 & $\mathbf{P}$ & 515 & $\mathbf{s}$ & $11-09-71$ \\
\hline 12S08W32DAAA01 & 6,320 & 03-02-68 & Ts & 240 & 6.0 & $\mathbf{P}$ & 215 & $\mathbf{s}$ & $10-08-91$ \\
\hline 12S08W34^AAC01 & 6,265 & -- & Qal & 45 & -. & - & - & $\mathbf{H}$ & 08-05-92 \\
\hline 13S09W01BCCA01 & 6,035 & $11-01-78$ & Ts & 97 & 6.0 & $\mathbf{P}$ & 86 & $\mathbf{H}$ & $10-09-91$ \\
\hline 13S09W04DCDC01 & 6,005 & $06-26-75$ & Ts & 121 & 6.0 & $\mathbf{p}$ & 90 & $\mathbf{H}$ & $10-09-91$ \\
\hline 13S09W17CBAA01 & 6,120 & $08-26-80$ & Ts & 119 & 6.0 & $\mathbf{P}$ & 110 & $\mathbf{S}$ & $10-08-91$ \\
\hline 13S09W21DDAD01 & 6,065 & -- & Qal & 54 & 6.0 & - & -- & $\mathbf{H}$ & $10-09-91$ \\
\hline 13S09W22DDDC01 & 6,057 & -- & Qal & 57 & -- & - & -- & $\mathrm{U}$ & $10-09-91$ \\
\hline 13S09W24BDBB01 & 6,075 & $-.92^{3}$ & Qal & 48 & 20.5 & - & -- & H & $10-09-91$ \\
\hline 13S09W34BDA 01 & 6,160 & $06-04-62$ & Ts & 146 & 4.0 & $\mathrm{o}$ & -- & $\mathbf{S}$ & $10-09-91$ \\
\hline 13S08W 19CDBD01 & 6,135 & $-\quad-80$ & QTKe & 78 & 5.0 & - & -- & $\mathbf{H}$ & $10-10-91$ \\
\hline 13S08W30BCD 01 & 6,115 & $02-05-88$ & Qal & 60 & 6.0 & $\mathbf{P}$ & 53 & $\mathbf{s}$ & $10-10-91$ \\
\hline 14S09W13ADC 01 & 6,380 & $06-22-89$ & Ts & 236 & 6.0 & $\mathbf{P}$ & 225 & $\mathbf{H}$ & $10-09-91$ \\
\hline 14S08W04CCDA01 & 6,237 & $09-17-66$ & Ts & 192 & 6.0 & $\mathbf{P}$ & 180 & $\mathbf{H}$ & $10-09-91$ \\
\hline 14S08W05ACB 01 & 6,222 & $05-04-90$ & Ts & 200 & 6.0 & O & -- & $\mathbf{H}$ & $10-08-91$ \\
\hline
\end{tabular}

$\begin{array}{llll}\text { 25N18W13CDDC01 } & 3,220 & 06-11-87 & \text { Qg } \\ \text { 25N1 8W14DBB 01 } & 3,078 & 05-06-89 & \text { Qal } \\ \text { 25N18W35ADDA01 } & 3,100 & 11-01-89 & \text { Qg } \\ \text { 25N18W35DBCB01 } & 3,105 & 11-03-89 & \text { Qg } \\ \text { 24N17W07BCAA01 } & 3,118 & 11-08-82 & \text { Qal }\end{array}$

Swan Valley

${ }^{3} 1892$ 
Table 1. Records of selected wells by basin, Northern Rocky Mountains intermontane basins, Montana (Continued)

\begin{tabular}{|c|c|c|c|c|c|c|c|c|c|}
\hline $\begin{array}{c}\text { Water lovel } \\
\text { (ft) }\end{array}$ & $\begin{array}{c}\text { Dis- } \\
\text { charge } \\
\text { (gall } \\
\text { min) }\end{array}$ & $\begin{array}{c}\text { Specific } \\
\text { capaclty } \\
\text { [(gai/ } \\
\text { min)/ft] }\end{array}$ & $\begin{array}{c}\text { Source } \\
\text { of dis- } \\
\text { charge } \\
\text { data }\end{array}$ & $\begin{array}{l}\text { Type } \\
\text { of log } \\
\text { avail- } \\
\text { able }\end{array}$ & $\begin{array}{c}\text { Date } \\
\text { water- } \\
\text { quallty } \\
\text { parameler } \\
\text { measured }\end{array}$ & $\begin{array}{l}\text { Specific } \\
\text { conduc- } \\
\text { tance } \\
(\mu \mathrm{S} / \mathrm{cm})\end{array}$ & $\begin{array}{c}\text { PH } \\
\text { (stan- } \\
\text { dard } \\
\text { units) }\end{array}$ & $\begin{array}{l}\text { Tem. } \\
\text { per- } \\
\text { sture } \\
\left({ }^{\circ} \mathrm{C}\right)\end{array}$ & Woli number \\
\hline \multicolumn{10}{|c|}{ Plains Valley-Continued } \\
\hline 19.63 & 5 & 6.8 & $\mathbf{S}$ & - & 09-05-91 & 69 & 6.9 & 10.0 & 20N26W24DDC 01 \\
\hline 15.00 & 500 & 38 & D & D & -- & -- & -- & -- & $20 \mathrm{~N} 26 \mathrm{~W} 27 \mathrm{DBBC} 01$ \\
\hline 19.58 & 600 & 100 & D & D & 09-03-91 & 88 & 7.3 & 13.5 & 20N26W27DDBC0I \\
\hline 67.18 & 6 & .7 & D & D & $09-03-91$ & 296 & 6.7 & 14.0 & 19N26W01CAB 01 \\
\hline 129.47 & 15 & 5.0 & D & $\mathbf{D}$ & $09-04-91$ & 75 & 7.2 & 9.0 & 19N26W03ACBC01 \\
\hline 147.88 & 1 & -- & D & $\mathbf{D}$ & $09-04-91$ & 168 & 7.9 & 12.0 & 19N26W03BDAC01 \\
\hline \multicolumn{10}{|c|}{ Red Rock Valley } \\
\hline 53.88 & 10 & 5.0 & D & D & $10-07-91$ & 1,000 & - & 10.5 & 10S10W29DCCC01 \\
\hline 86.30 & 60 & 13 & D & $\mathbf{D}$ & - & -- & - & - & 11S10W26BBCC01 \\
\hline 126.98 & 40 & 2.7 & D & D & -- & -- & - & -- & 11S10W27ADAC01 \\
\hline$* 387$ & 15 & -- & D & $\mathbf{D}$ & -- & -- & - & -- & 11S10W34CCACO1 \\
\hline 12.27 & 20 & 2.9 & $\mathbf{D}$ & D & -- & $\cdots$ & -- & $\cdots$ & 11S08W12CCBA01 \\
\hline 25.43 & 6 & -- & D & D & -- & - & -- & -- & 11S08W22ABBD01 \\
\hline 60.20 & $\cdots$ & -- & - & - & -- & -- & -- & -- & 11S08W32ACAC01 \\
\hline$* 511$ & - & -- & - & $\mathbf{D}$ & - & -- & -- & -. & 12S10W22CADA01 \\
\hline 179.31 & 30 & 3.0 & D & D & -- & -- & -- & - & 12S 08W03CBBD01 \\
\hline *475 & 10 & .4 & $\mathbf{D}$ & D & - & -- & -- & -. & $12 \mathrm{~S} 08 \mathrm{~W} 31 \mathrm{ABD} 01$ \\
\hline 38.06 & 23 & .9 & $\mathbf{D}$ & D & - & -- & -- & -- & 12S08W32DAAA01 \\
\hline 10.80 & $\sim$ & -- & - & - & 08-05-92 & 613 & 7.5 & 8.5 & 12S08W34AAAC01 \\
\hline 40.11 & 20 & 4.0 & D & D & $10-09-91$ & 426 & 7.6 & 9.0 & 13S09W01BCCA01 \\
\hline 73.35 & 25 & 1.7 & D & D & $10-09-91$ & 1,500 & 7.1 & 9.0 & 13S09W04DCDC01 \\
\hline 107.26 & 15 & -- & $\mathrm{D}$ & D & - & $\cdots$ & $-\cdot$ & $\cdots$ & 13S09W17CBAA01 \\
\hline 27.30 & -- & -- & - & - & -- & -- & -- & -. & 13S09W21DDAD01 \\
\hline 6.42 & -- & -- & - & - & -- & -- & -- & -- & 13S09W22DDDC01 \\
\hline 14.34 & 30 & -- & D & D & $10-09-91$ & 492 & 7.5 & 12.0 & 13S09W24BDBB0I \\
\hline 84.15 & 16 & 1.5 & D & D & $10-09-91$ & 657 & 7.8 & 8.0 & 13S09W34BDA 01 \\
\hline 55.35 & - & -- & - & - & -- & - & -- & -- & 13S08W19CDBD01 \\
\hline 52.23 & 20 & 2.9 & D & D & $10-10-91$ & 502 & 7.3 & 12.5 & 13S08W30BCD 01 \\
\hline 201.68 & 22 & -- & D & D & $10-09-91$ & 633 & -- & 10.0 & 14S09W13ADC 01 \\
\hline 145.03 & 50 & 3.3 & D & D & -- & -- & - & - & 14S08W04CCDA01 \\
\hline 133.60 & 20 & 2.0 & D & - & $10-08-91$ & 587 & 7.5 & 10.5 & 14S08W05ACB 01 \\
\hline \multicolumn{10}{|c|}{ Swan Valley } \\
\hline 83.63 & 15 & .1 & D & D & $09-25-91$ & 456 & -- & 9.0 & $25 \mathrm{~N} 18 \mathrm{~W} 13 \mathrm{CDDCO} 1$ \\
\hline 9.07 & 25 & 6.3 & D & D & $09-25-91$ & 378 & -- & 7.0 & 25N18W14DBB 01 \\
\hline 12.76 & 100 & 1.6 & D & D & -- & -- & -- & $\cdots$ & 25N18W35ADDA01 \\
\hline 23.31 & 100 & 1.5 & D & D & - & - & -- & $\cdots$ & 25N18W35DBCB01 \\
\hline .60 & 12 & .4 & $\mathbf{S}$ & D & $09-27-91$ & 348 & -- & 8.0 & 24N17W07BCAA01 \\
\hline
\end{tabular}


Table 1. Records of selected wells by basin. Northern Rocky Mountains intermontane basins, Montana (Continued)

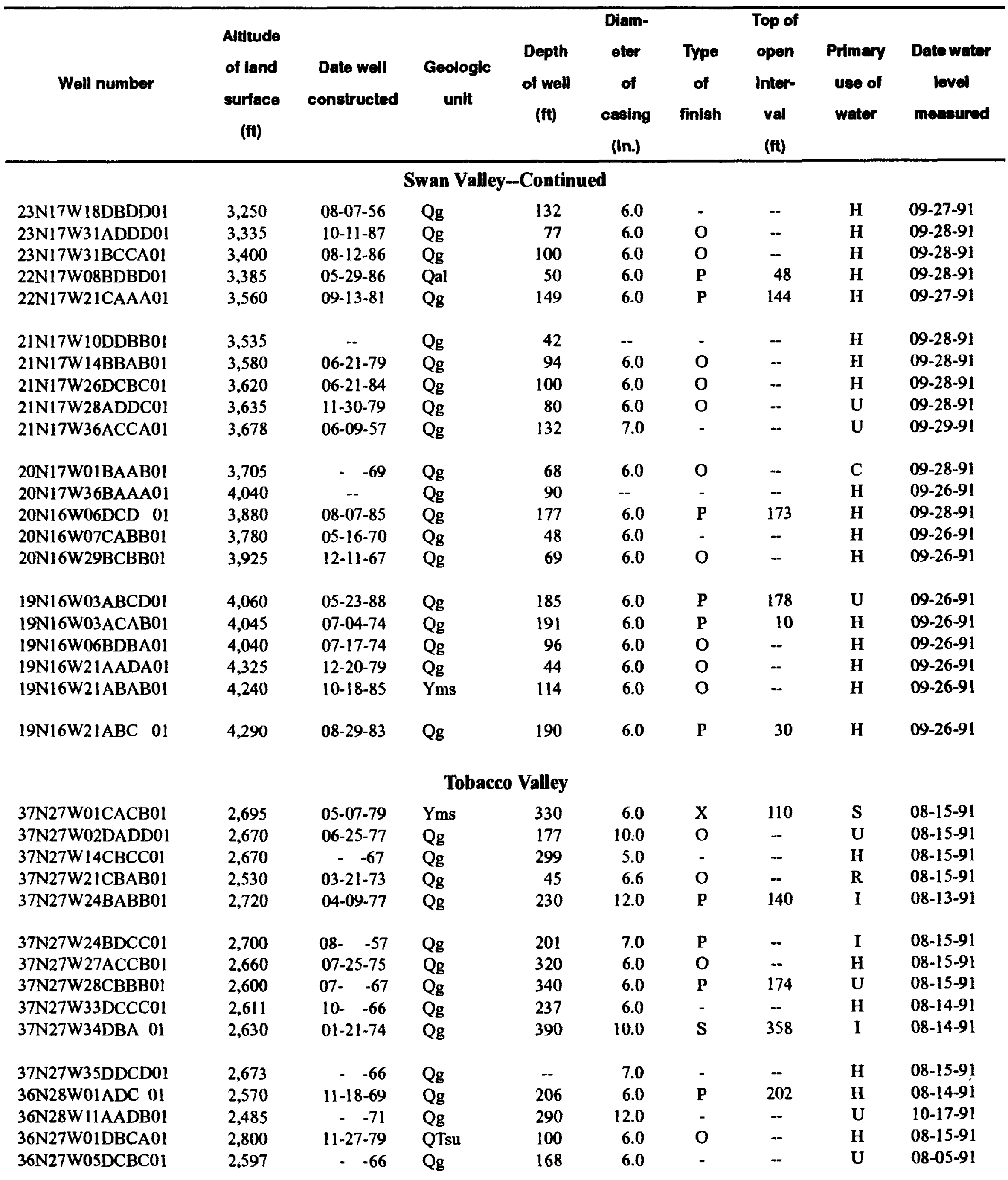


Table 1. Records of selected wells by basin, Northern Rocky Mountains intermontane basins, Montana (Continued)

\begin{tabular}{|c|c|c|c|c|c|c|c|c|c|}
\hline $\begin{array}{c}\text { Water level } \\
\text { (ft) }\end{array}$ & $\begin{array}{c}\text { Dis- } \\
\text { charge } \\
\text { (gal/ } \\
\text { min) }\end{array}$ & $\begin{array}{c}\text { Specific } \\
\text { capacity } \\
\text { [(gal/ } \\
\text { min)/ft] }\end{array}$ & $\begin{array}{c}\text { Source } \\
\text { of dis- } \\
\text { charge } \\
\text { data }\end{array}$ & $\begin{array}{l}\text { Type } \\
\text { of log } \\
\text { avall- } \\
\text { able }\end{array}$ & $\begin{array}{c}\text { Date } \\
\text { water- } \\
\text { quality } \\
\text { parameter } \\
\text { measured }\end{array}$ & $\begin{array}{c}\text { Spechic } \\
\text { conduc- } \\
\text { tance } \\
(\mu S / \mathrm{cm})\end{array}$ & $\begin{array}{c}\text { pH } \\
\text { (stan- } \\
\text { dard } \\
\text { units) }\end{array}$ & $\begin{array}{l}\text { Tem- } \\
\text { per- } \\
\text { ature } \\
\left({ }^{\circ} \mathrm{C}\right)\end{array}$ & Well number \\
\hline
\end{tabular}

\begin{tabular}{|c|c|c|c|c|c|c|c|c|c|}
\hline \multicolumn{10}{|c|}{ Swan Valley-Continued } \\
\hline+14.09 & 100 & -. & 0 & - & $09-27-91$ & 418 & -- & 7.0 & 23N17W 18DBDD01 \\
\hline 2.50 & 15 & 8.7 & $\mathbf{S}$ & D & $09-28-91$ & 366 & -- & 7.5 & 23N17W31ADDD01 \\
\hline 19.42 & 10 & -- & $\mathrm{D}$ & D & $\infty 9-28-91$ & 200 & -- & 6.5 & 23N17W31BCCA01 \\
\hline+4.95 & 12 & 1.2 & $\mathbf{S}$ & D & $09-28-91$ & 278 & -- & 6.5 & 22N17W08BDBD01 \\
\hline 132.21 & 12 & 4.0 & $\mathbf{D}$ & $\mathbf{D}$ & $09-27-91$ & 376 & $\sim$ & 7.5 & 22N17W21CAAA01 \\
\hline 15.12 & 50 & -. & D & - & $09-28-91$ & 259 & -- & 7.5 & 21N17W10DDBB01 \\
\hline 15.74 & 7 & 2.1 & $\mathbf{S}$ & D & $09-28-91$ & 368 & -- & 7.5 & $21 \mathrm{~N} 17 \mathrm{~W} 14 \mathrm{BBAB} 01$ \\
\hline 13.68 & 10 & .4 & $\mathbf{S}$ & D & $09-28-91$ & 153 & -- & 7.5 & $21 \mathrm{~N} 17 \mathrm{~W} 26 \mathrm{DCBC} 01$ \\
\hline 8.97 & 9 & .2 & D & D & -- & -- & - & -. & 21N17W28ADDC01 \\
\hline 56.72 & 14 & -- & $\mathbf{D}$ & $\mathbf{D}$ & -- & -- & -- & -- & 21N17W36ACCA01 \\
\hline 25.85 & 15 & 2.0 & $\mathbf{s}$ & - & $09-28-91$ & 323 & -- & 8.0 & 20N17W01BAAB01 \\
\hline 45.50 & 9 & .3 & $\mathrm{~s}$ & - & $09-26-91$ & 429 & -- & 7.0 & 20N17W36BAAA01 \\
\hline 59.43 & 15 & -- & D & D & $09-26-91$ & 450 & -- & 8.5 & 20N16W06DCD 01 \\
\hline 24.66 & - & -- & - & - & $09-26-91$ & 397 & - & 7.0 & 20N16W07CABB01 \\
\hline 18.22 & 3 & .8 & $\mathbf{S}$ & - & $09-26-91$ & 501 & - & 8.5 & 20N16W29BCBB01 \\
\hline+1.91 & 5 & .2 & $\mathrm{D}$ & $\mathrm{D}$ & -- & -- & -- & -- & 19N16W03ABCD01 \\
\hline 9.47 & 50 & -- & D & $\mathbf{D}$ & -- & -- & -- & -- & 19N16W03ACAB01 \\
\hline 12.39 & 20 & .6 & D & D & - & - & -- & -- & 19N16W06BDBA01 \\
\hline 14.10 & 10 & .4 & $\mathrm{D}$ & D & $09-26-91$ & 628 & -- & 7.0 & 19N16W21AADA01 \\
\hline 41.45 & 20 & 1.2 & $\mathrm{D}$ & $\mathbf{D}$ & $09-26-91$ & 288 & -- & 7.5 & $19 \mathrm{~N} 16 \mathrm{~W} 21 \mathrm{ABAB} 01$ \\
\hline *149 & $<1$ & -- & $\mathrm{D}$ & $\mathbf{D}$ & $09-26-91$ & 199 & - & 7.5 & $19 \mathrm{~N} 16 \mathrm{~W} 21 \mathrm{ABC} 01$ \\
\hline \multicolumn{10}{|c|}{ Tobacco Valley } \\
\hline+5.00 & 30 & .1 & D & D & $08-15-91$ & 401 & - & 11.0 & 37N27W01CACB01 \\
\hline 52.79 & -- & - & - & D & -- & -- & -- & -- & 37N27W02DADD01 \\
\hline 71.93 & -- & -- & - & D & $08-15-91$ & 280 & -- & 11.0 & $37 \mathrm{~N} 27 \mathrm{~W} 14 \mathrm{CBCC} 01$ \\
\hline 9.57 & -- & -- & - & D & $06-25-73$ & -- & - & 15.0 & $37 \mathrm{~N} 27 \mathrm{~W} 21 \mathrm{CBAB} 01$ \\
\hline 110.99 & 340 & 9.4 & $\mathbf{S}$ & D & -- & - & -- & -- & $37 \mathrm{~N} 27 \mathrm{~W} 24 \mathrm{BABB} 01$ \\
\hline 119.07 & -- & -- & - & - & -- & -- & -- & -. & $37 \mathrm{~N} 27 \mathrm{~W} 24 \mathrm{BDCCO}$ \\
\hline 138.48 & 60 & .6 & D & $\mathrm{D}$ & -- & -- & -- & -- & $37 \mathrm{~N} 27 \mathrm{~W} 27 \mathrm{ACCB} 01$ \\
\hline 90.05 & -- & -- & - & - & -- & -- & $\cdots$ & -- & $37 \mathrm{~N} 27 \mathrm{~W} 28 \mathrm{CBBB01}$ \\
\hline 61.11 & 9 & .3 & $\mathbf{s}$ & D & 08-14-91 & 513 & -- & 13.0 & 37N27W33DCCC01 \\
\hline 91.78 & 50 & -- & $\mathrm{D}$ & $\mathrm{D}$ & -- & $\cdots$ & - & - & 37N27W34DBA 0I \\
\hline 132.22 & -- & -- & - & - & 08-15-91 & 363 & -. & 10.5 & 37N27W35DDCD01 \\
\hline 104.01 & 15 & .4 & D & D & $08-14-91$ & 505 & -- & 12.5 & 36N28W01ADC 01 \\
\hline 40.36 & -- & -- & - & - & -- & -- & -- & $\omega$ & $36 \mathrm{~N} 28 \mathrm{~W} 11 \mathrm{AADB} 01$ \\
\hline 74.06 & 30 & -- & D & D & - & -- & -- & -- & 36N27W01DBCA01 \\
\hline 35.12 & -- & -- & - & - & $08-15-91$ & 817 & - & 11.5 & $36 \mathrm{~N} 27 \mathrm{~W} 05 \mathrm{DCBC} 01$ \\
\hline
\end{tabular}


Table 1. Records of selected wells by basin, Northem Rocky Mountains intermontane basins, Montana (Continued)

\begin{tabular}{|c|c|c|c|c|c|c|c|c|c|}
\hline Woll number & $\begin{array}{l}\text { Altitude } \\
\text { of land } \\
\text { surface } \\
\text { (fi) }\end{array}$ & $\begin{array}{l}\text { Date well } \\
\text { constructed }\end{array}$ & $\begin{array}{c}\text { Geologic } \\
\text { unit }\end{array}$ & $\begin{array}{l}\text { Depth } \\
\text { of well } \\
\text { (ft) }\end{array}$ & $\begin{array}{c}\text { Diam- } \\
\text { oter } \\
\text { of } \\
\text { caolng } \\
\text { (In.) } \\
\end{array}$ & $\begin{array}{l}\text { Type } \\
\text { of } \\
\text { finish }\end{array}$ & $\begin{array}{l}\text { Top of } \\
\text { open } \\
\text { inter. } \\
\text { val } \\
\text { (ft) }\end{array}$ & $\begin{array}{l}\text { Primary } \\
\text { use of } \\
\text { water }\end{array}$ & $\begin{array}{c}\text { Date water } \\
\text { level } \\
\text { measured }\end{array}$ \\
\hline \multicolumn{10}{|c|}{ Tobacco Valley-Continued } \\
\hline 36N27W14DCCB01 & 2,570 & $07-10-61$ & Qal & 88 & 14.0 & $\mathbf{s}$ & 78 & $\mathbf{u}$ & $08-15-91$ \\
\hline $36 \mathrm{~N} 27 \mathrm{~W} 23 \mathrm{AAC}$ 0I & 2,570 & $08-01-72$ & Qal & 20 & 84.0 & $\mathbf{H}$ & -. & $\mathbf{P}$ & $08-15-91$ \\
\hline 36N27W23DDDD01 & 2,720 & -. & QTsu & 91 & 6.0 & - & -- & $\mathrm{U}$ & $08-12-91$ \\
\hline 36N26W22CBAA01 & 2,978 & $04-25-86$ & QTsu & 125 & 6.0 & o & -- & $\mathrm{H}$ & 08-12-91 \\
\hline 36N26W22DBBC01 & 3,020 & $08-27-74$ & QTsu & 80 & 6.0 & $\mathbf{P}$ & - & $\mathbf{H}$ & $08-12-91$ \\
\hline 36N25W05CCBA01 & 3,340 & -- & QTsu & -- & 6.0 & - & -- & $\mathbf{H}$ & $08-10-91$ \\
\hline 35N27W05CACD01 & 3,295 & 09-17-77 & Yms & 126 & -- & 0 & -- & $\mathbf{H}$ & 08-13-91 \\
\hline 35N27W05CBA 01 & 3,260 & 03-26-78 & QTsu & 145 & 6.0 & o & -- & $\mathbf{H}$ & 08-13-91 \\
\hline 35N27W06DAAD01 & 3,355 & $04-03-75$ & QTsu & 75 & 6.0 & $\mathbf{P}$ & -- & $\mathbf{H}$ & -- \\
\hline 35N26W08ABAA01 & 2,856 & $12-14-81$ & QTsu & 180 & 6.0 & $\mathbf{O}$ & -- & $\mathbf{H}$ & $08-11-91$ \\
\hline 35N26W08ADAC01 & 2,788 & $10-16-72$ & QTsu & 248 & 6.0 & $\mathbf{P}$ & 244 & $\mathbf{H}$ & $08-11-91$ \\
\hline 35N26W12ACBB01 & 2,965 & -- & Qal & 26 & -- & - & - & H & $08-11-91$ \\
\hline 35N26W 14BDDC01 & 2,864 & $10-27-80$ & QTsu & 42 & 6.0 & $\mathbf{O}$ & -- & $\mathbf{H}$ & $08-10-91$ \\
\hline 35N26W14CABD01 & 2,858 & $07-28-85$ & QTsu & 60 & 6.0 & O & - & $\mathbf{H}$ & $08-11-91$ \\
\hline 35N26W27CCAB0I & 3,038 & $08-11-88$ & Yms & 320 & 6.0 & $\mathrm{O}$ & -- & $\mathbf{H}$ & $08-10-91$ \\
\hline 35N26W27CCAD01 & 3,048 & $07-25-89$ & QTsu & 168 & 6.0 & 0 & -- & H & $08-11-91$ \\
\hline 35N25W32ADCB01 & 3,195 & $12-21-73$ & QTsu & 290 & 6.0 & $\mathbf{P}$ & 240 & H & 08-10-91 \\
\hline 34N26W04ACDB01 & 3,325 & $08-01-89$ & Yms & 235 & 6.0 & $\mathbf{P}$ & 178 & $\mathbf{H}$ & 08-09-91 \\
\hline 34N26W25DDAD01 & 3,280 & $08-29-79$ & QTsu & 111 & 6.0 & O & - & $\mathbf{H}$ & $08-08-91$ \\
\hline 34N25W07ACDB01 & 3,060 & $10-\quad-53$ & Yms & 199 & 6.0 & 0 & -- & $\mathbf{P}$ & $08-08-91$ \\
\hline 34N25W16ABCB01 & 3,122 & $07-21-86$ & QTsu & 120 & 6.0 & o & -- & $\mathbf{H}$ & $08-07-91$ \\
\hline 34N25W30BD 01 & 3,220 & $10-24-79$ & QTsu & 140 & 6.0 & o & -- & HI & 08-08-91 \\
\hline 34N25W30CAAA01 & 3,220 & $09-03-86$ & QTsu & 385 & 6.0 & o & -- & $\mathbf{H}$ & $08-08-91$ \\
\hline 33N26W14ACCB01 & 3,428 & $12-17-74$ & QTsu & 198 & 6.0 & 0 & -- & $\mathbf{H}$ & 08-09-91 \\
\hline 33N26W30ABAB01 & 3,880 & $05-12-80$ & QTsu & 120 & 6.0 & o & -- & $\mathbf{H}$ & $08-08-91$ \\
\hline \multicolumn{10}{|c|}{ Townsend Valley } \\
\hline 10N01W02CABB01 & 3,860 & $05-30-81$ & Ts & 103 & 6.6 & $\mathbf{P}$ & 83 & $\mathbf{H}$ & $04-24-91$ \\
\hline IONO1W02CAC 01 & 3,930 & $04-08-82$ & Ts & 275 & 6.6 & $\mathbf{P}$ & 215 & $\mathrm{H}$ & 04-23-91 \\
\hline 10N01W23CCADO1 & 3,835 & -- & Qal & 62 & - & - & - & $\mathbf{H}$ & $05-13-91$ \\
\hline 10N01E14BACA01 & 4,165 & $09-13-69$ & Ts & 321 & 6.6 & $\mathbf{P}$ & 280 & $\mathbf{H}$ & $04-23-91$ \\
\hline 10N01E22CBD 01 & 3,995 & $10-09-73$ & Ts & 380 & 16.0 & $\mathbf{P}$ & 160 & I & $04-25-91$ \\
\hline 10NO1E28АDCB01 & 3,935 & -- & Ts & 222 & - & - & -- & 1 & $04-25-91$ \\
\hline 10N01E28CABB01 & 3,925 & $10-06-74$ & Ts & 300 & 16.0 & $\mathbf{P}$ & 120 & I & $04-25-91$ \\
\hline 09N01E28AAA 01 & 3,830 & $10-28-81$ & Ts & 60 & 6.6 & $\mathbf{P}$ & 30 & $\mathbf{H}$ & 04-23-91 \\
\hline 09N02E16CCCD01 & 4,085 & -. & Ts & 100 & 6.0 & - & -- & H & 04-24-91 \\
\hline 09N02E16CDAD01 & 4,235 & $05-19-76$ & Yms & 340 & 6.6 & $\mathbf{x}$ & 340 & $\mathbf{H}$ & $04-24-91$ \\
\hline
\end{tabular}


Table 1. Records of selected wells by basin, Northern Rocky Mountains intermontane basins, Montana (Continued)

\begin{tabular}{|c|c|c|c|c|c|c|c|c|c|}
\hline $\begin{array}{c}\text { Water level } \\
\text { (fi) }\end{array}$ & $\begin{array}{c}\text { Dis- } \\
\text { charge } \\
\text { (gal/ } \\
\text { min) }\end{array}$ & $\begin{array}{c}\text { Specific } \\
\text { capacity } \\
\text { [(gal/ } \\
\text { min)/ft] }\end{array}$ & $\begin{array}{c}\text { Source } \\
\text { of dls- } \\
\text { charge } \\
\text { data }\end{array}$ & $\begin{array}{l}\text { Type } \\
\text { of log } \\
\text { avali- } \\
\text { able }\end{array}$ & $\begin{array}{l}\text { Date } \\
\text { water- } \\
\text { quallty } \\
\text { parameter } \\
\text { measured }\end{array}$ & $\begin{array}{c}\text { Specific } \\
\text { conduc- } \\
\text { tance } \\
(\mu \mathrm{S} / \mathrm{cm})\end{array}$ & $\begin{array}{l}\text { pH } \\
\text { (stan- } \\
\text { dard } \\
\text { Units) }\end{array}$ & $\begin{array}{l}\text { Tom- } \\
\text { por- } \\
\text { ature } \\
\left({ }^{\circ} \mathrm{C}\right)\end{array}$ & Well number \\
\hline
\end{tabular}

\begin{tabular}{|c|c|c|c|c|c|c|c|c|c|}
\hline \multicolumn{10}{|c|}{ Tobacco Valley_Continued } \\
\hline 5.95 & -- & -- & - & D & - & -- & - & -- & $36 \mathrm{~N} 27 \mathrm{~W} 14 \mathrm{DCCB} 01$ \\
\hline 7.07 & -- & -- & - & - & -- & -- & -- & -- & 36N27W23AAC 01 \\
\hline 83.19 & -. & -- & - & - & -- & -- & -. & -- & 36N27W23DDDD01 \\
\hline Flowing & 60 & -- & D & D & -- & -- & -- & -. & $36 \mathrm{~N} 26 \mathrm{~W} 22 \mathrm{CBAA01}$ \\
\hline 21.53 & 4 & .1 & D & D & -- & -- & -- & -- & $36 \mathrm{~N} 26 \mathrm{~W} 22 \mathrm{DBBC} 01$ \\
\hline 360.92 & -- & -- & - & - & - & -- & - & -- & $36 \mathrm{~N} 25 \mathrm{~W} 05 \mathrm{CCBA01}$ \\
\hline 11.89 & -- & -. & - & D & -- & -- & - & -- & $35 \mathrm{~N} 27 \mathrm{~W} 05 \mathrm{CACD} 01$ \\
\hline Flowing & .. & -- & - & D & -- & -- & -- & -- & 35N27W05CBA 01 \\
\hline-- & 30 & 1.0 & D & D & -- & -- & -- & -- & 35N27W06DAAD0 I \\
\hline 97.95 & 10 & .2 & D & $\mathbf{D}$ & -- & -- & - & -- & 35N26W08ABAA01 \\
\hline 32.87 & 10 & .1 & D & $\mathbf{D}$ & - & -- & -- & -- & 35N26W08ADAC01 \\
\hline 13.23 & -- & -. & - & - & -- & -- & -- & -- & $35 \mathrm{~N} 26 \mathrm{~W} 12 \mathrm{ACBB} 01$ \\
\hline 10.81 & 25 & 1.0 & D & $D$ & -- & -- & -- & -- & $35 \mathrm{~N} 26 \mathrm{~W} 14 \mathrm{BDDC01}$ \\
\hline 3.00 & 25 & .4 & $\mathrm{D}$ & D & - & - & $\ldots$ & -. & $35 \mathrm{~N} 26 \mathrm{~W} 14 \mathrm{CABDO1}$ \\
\hline 132.42 & 15 & .1 & D & $\mathbf{D}$ & -- & -- & -- & -. & $35 \mathrm{~N} 26 \mathrm{~W} 27 \mathrm{CCABO1}$ \\
\hline 144.68 & 15 & -- & D & D & -. & -- & - & .. & 35N26W27CCAD01 \\
\hline 242.12 & 20 & 1.8 & D & $\mathrm{D}$ & -- & -- & - & -. & $35 \mathrm{~N} 25 \mathrm{~W} 32 \mathrm{ADCB} 0 \mathrm{I}$ \\
\hline 56.48 & 17 & .2 & D & D & -- & -- & -- & - & 34N26W04ACDB01 \\
\hline 53.20 & 7 & .2 & D & D & -. & -- & -- & -- & 34N26W25DDAD01 \\
\hline 82.97 & 14 & .3 & D & $\mathbf{D}$ & - & -- & -- & -- & 34N25W07ACDB01 \\
\hline 30.83 & 20 & .3 & D & D & -- & -- & -- & -- & $34 \mathrm{~N} 25 \mathrm{~W} 16 \mathrm{ABCB} 01$ \\
\hline 126.57 & 3 & .8 & D & D & -- & -- & - & -- & 34N25W30BD 01 \\
\hline 132.36 & 20 & .5 & D & $\mathbf{D}$ & -- & -- & -- & -- & 34N25W30CAAA01 \\
\hline 41.04 & 70 & .6 & D & $\mathbf{D}$ & -- & -- & -- & -- & $33 \mathrm{~N} 26 \mathrm{~W} 14 \mathrm{ACCB} 01$ \\
\hline 33.90 & 5 & .1 & D & $\mathbf{D}$ & -- & -- & -- & -- & $33 \mathrm{~N} 26 \mathrm{~W} 30 \mathrm{ABAB} 01$ \\
\hline \multicolumn{10}{|c|}{ Townsend Valley } \\
\hline 69.40 & 8 & 8.2 & $\mathbf{s}$ & $D$ & $04-24-91$ & 586 & 7.4 & 10.5 & 10N01W02CABBOI \\
\hline 149.50 & 30 & .3 & D & D & $04-23-91$ & 644 & 7.5 & 11.5 & 10N01W02CAC 01 \\
\hline 34.75 & 11 & -- & $\mathbf{s}$ & - & $05-13-91$ & 1,620 & 7.7 & 10.5 & 10N01W23CCAD01 \\
\hline 314.42 & 4 & $\cdots$ & $\mathbf{s}$ & $\mathbf{D}$ & 04-23-91 & 601 & 7.5 & 10.5 & 10NOIE14BACA01 \\
\hline 150.71 & 2,400 & 120 & D & $\mathrm{D}$ & - & -- & -- & $\cdots$ & 10NO1E22CBD 01 \\
\hline$* 96$ & -- & $\because$ & - & $D$ & -- & -. & - & - & 10N01E28ADCB01 \\
\hline 121.83 & 1,600 & 34 & D & $\mathrm{D}$ & -. & -- & -- & -. & 10N01E28CABB01 \\
\hline 9.90 & 12 & 3.6 & $\mathbf{s}$ & $\mathrm{D}$ & $04-23-91$ & 1,320 & 7.2 & 9.5 & 09N01E28AAA 01 \\
\hline 68.14 & 9 & - & $s$ & - & 04-24-91 & 469 & 8.1 & 4.0 & 09N02E16CCCD01 \\
\hline 94.99 & 6 & .4 & D & $\mathrm{D}$ & -- & - & -- & -- & 09N02E16CDAD01 \\
\hline
\end{tabular}


Table 1. Records of selected wells by basin, Northern Rocky Mountains intermontane basins, Montana (Continued)

\begin{tabular}{|c|c|c|c|c|c|c|c|c|c|}
\hline Well number & $\begin{array}{l}\text { Altitude } \\
\text { of land } \\
\text { surface } \\
\text { (ft) }\end{array}$ & $\begin{array}{l}\text { Date well } \\
\text { constructed }\end{array}$ & $\begin{array}{c}\text { Goologle } \\
\text { unlt }\end{array}$ & $\begin{array}{l}\text { Depth } \\
\text { of weil } \\
\text { (ft) }\end{array}$ & $\begin{array}{c}\text { Diam- } \\
\text { eter } \\
\text { of } \\
\text { casing } \\
\text { (in.) }\end{array}$ & $\begin{array}{c}\text { Type } \\
\text { of } \\
\text { finlsh }\end{array}$ & $\begin{array}{c}\text { Top of } \\
\text { open } \\
\text { Inter- } \\
\text { val } \\
\text { (ft) }\end{array}$ & $\begin{array}{l}\text { Primary } \\
\text { uae of } \\
\text { water }\end{array}$ & $\begin{array}{c}\text { Date wate } \\
\text { lovel } \\
\text { measure }\end{array}$ \\
\hline \multicolumn{10}{|c|}{ Townsend Valley-Continued } \\
\hline 09N02E34DAAA01 & 4,205 & $10-12-84$ & Qal & 182 & 6.0 & $\mathbf{P}$ & 117 & $\mathbf{H}$ & $05-14-91$ \\
\hline 08N02E14BBBC01 & 4,085 & $06-01-88$ & Ts & 140 & 6.0 & o & -- & $\mathbf{H}$ & $05-13-91$ \\
\hline 08N02E23CBBA01 & 3,975 & $11-27-73$ & Ts & 240 & 6.0 & $\mathbf{P}$ & 40 & $\mathbf{U}$ & $05-14-91$ \\
\hline 08N02E35BCDD01 & 3,925 & $06-28-76$ & Ts & 95 & 6.0 & $\mathbf{P}$ & 77 & $\mathbf{H}$ & $05-15-91$ \\
\hline 07N01E03CDDD01 & 3,975 & $02-25-87$ & Ts & 221 & 6.0 & $\mathbf{P}$ & 211 & $\mathbf{H}$ & $05-30-91$ \\
\hline 07N01E03CDDD02 & 3,978 & $01-05-78$ & Ts & 170 & 6.0 & o & -- & $\mathbf{U}$ & $05-30-91$ \\
\hline 07N02E03ADD 01 & 3,895 & $01-22-74$ & Ts & 75 & 6.0 & $\mathbf{P}$ & 70 & $\mathbf{H}$ & $05-15-91$ \\
\hline 07N02E15CBAB01 & 3,880 & $09-18-86$ & Qal & 99 & 6.0 & o & - & $\mathbf{H}$ & $05-14-91$ \\
\hline 07N02E29CABC0I & 3,819 & $12-04-68$ & Qal & 32 & 6.0 & $\mathbf{P}$ & 28 & $\mathbf{H}$ & -- \\
\hline 07N02E31ABAA01 & 3,820 & $05-14-70$ & Qal & 93 & 12.0 & $\mathbf{s}$ & 55 & $\mathbf{P}$ & -- \\
\hline 07N02E31ADDA01 & 3,825 & $08-17-60$ & Qal & 60 & 13.0 & $\mathbf{S}$ & 37 & $\mathbf{P}$ & -- \\
\hline 07N02E31DBBA01 & 3,820 & $10-20-58$ & Qal & 50 & 12.0 & $\mathbf{s}$ & 30 & $\mathbf{P}$ & $05-29-91$ \\
\hline 07N02E34ABBD01 & 4,065 & $04-16-82$ & Ts & 200 & 6.0 & G & 180 & $\mathbf{H}$ & $05-15-91$ \\
\hline 07N03E07BBBC01 & 4,175 & $01-13-78$ & Ts & 145 & 6.0 & $\mathbf{P}$ & 105 & $\mathbf{H}$ & $05-15-91$ \\
\hline O7N03E25DABB01 & 4,720 & $01-20-80$ & Ts & 305 & 6.0 & $\mathbf{P}$ & 180 & $\mathbf{H}$ & $05-30-91$ \\
\hline 06N01E32DBB $\Lambda 01$ & 4,475 & $08-21-78$ & Ts & 235 & 6.0 & o & -. & $\mathbf{H}$ & $05-31-91$ \\
\hline 06N02E09BACD01 & 3,875 & --20 & Ts & 110 & 4.0 & - & -- & $\mathbf{H}$ & $10-21-92$ \\
\hline 06N02E23 АBAB01 & 4,060 & $08-29-74$ & Ts & 400 & 6.0 & $\mathbf{P}$ & 180 & I & -- \\
\hline 06N02E23DBCA01 & 4,060 & $02-16-85$ & Ts & 400 & 16.0 & $\mathbf{P}$ & 140 & I & $05-29-91$ \\
\hline 05N01E27BBCC01 & 4,150 & $02-28-73$ & Ts & 305 & 16.0 & $\mathbf{P}$ & 100 & I & -- \\
\hline 05N01E27BDA 01 & 4,115 & $03--72$ & Ts & 354 & 16.0 & $\mathbf{P}$ & 50 & $\mathbf{I}$ & $05-01-91$ \\
\hline 05N01E27CCBB01 & 4,143 & $03-17-62$ & Ts & 215 & 16.0 & $\mathbf{F}$ & 120 & I & $09-26-91$ \\
\hline 05N01E27CDAA01 & 4,110 & $11-28-77$ & Ts & 302 & 16.0 & $\mathbf{P}$ & 70 & I & $05-01-91$ \\
\hline 05N01E28BCAA01 & 4,235 & 09-09-78 & Ts & 340 & 16.0 & $\mathbf{P}$ & 152 & I & $05-17-91$ \\
\hline 05N01E33BACD01 & 4,177 & $04-15-67$ & Ts & 252 & 20.0 & $\mathbf{F}$ & 152 & I & $04-30-91$ \\
\hline 05N01E34CCCC01 & 4,135 & $04-04-77$ & Ts & 325 & 16.0 & $\mathbf{F}$ & 90 & I & $04-30-91$ \\
\hline 05N01E34DCCD01 & 4,095 & -- & Ts & 108 & -- & - & -- & I & $04-30-91$ \\
\hline 05N02E03 ACDC01 & 3,905 & $06-25-82$ & Qal & 58 & 6.0 & O & -- & $\mathbf{S}$ & $10-19-92$ \\
\hline 05N02E05BDCB01 & 3,894 & -- & Qal & 30 & -- & - & -- & $\mathbf{H}$ & - \\
\hline 05N02E10ADDD01 & 3,923 & $06-22-89$ & Qal & 63 & 6.0 & O & - & $\mathbf{H}$ & $05-29-91$ \\
\hline 05N02E22CBBA01 & 3,915 & $03-20-81$ & Ts & 40 & 6.0 & o & -- & $\mathbf{H}$ & $10-20-92$ \\
\hline 05N02E23DBCA01 & 3,930 & $04-28-82$ & Qal & 56 & 6.0 & $\mathrm{O}$ & -- & $\mathbf{H}$ & $10-19-92$ \\
\hline 05N02E31DDCB01 & 3,985 & $11-21-80$ & Ts & 35 & 6.0 & $\mathbf{P}$ & 63 & $\mathrm{U}$ & -91 \\
\hline 05N02E33DACA01 & 4,021 & -- & Ts & 148 & -. & - & -- & $\mathbf{H}$ & -- \\
\hline 05N02E34AADD01 & 3,982 & $04-30-77$ & Ts & 226 & 16.0 & $\mathbf{F}$ & 85 & $\mathbf{U}$ & $05-02-91$ \\
\hline 04N01E02BBCC01 & 4,075 & $05-28-66$ & Ts & 191 & 16.0 & $\mathbf{F}$ & 100 & I & $04-30-91$ \\
\hline 04N0 1E02CCBB01 & 4,090 & 03-29-74 & Ts & 275 & 16.0 & $\mathbf{P}$ & 117 & I & 04-30-91 \\
\hline 04N01E02CCCB01 & 4,090 & $10-21-64$ & Ts & 250 & 20.0 & $\mathbf{F}$ & 97 & I & $04-30-91$ \\
\hline O4N01E03BABA0I & 4,115 & $02-27-73$ & Ts & 220 & 6.0 & o & - & $\mathrm{U}$ & $04-30-91$ \\
\hline 04N01E09ADB 01 & 4,175 & $03-12-76$ & Ts & 349 & 16.0 & $\mathbf{P}$ & 150 & $\mathbf{I}$ & $05-01-91$ \\
\hline
\end{tabular}


Table 1. Records of selected wells by basin, Northern Rocky Mountains intermontane basins, Montana (Continued)

\begin{tabular}{|c|c|c|c|c|c|c|c|c|c|}
\hline $\begin{array}{l}\text { Water lovol } \\
\text { (ft) }\end{array}$ & $\begin{array}{c}\text { Dls- } \\
\text { charge } \\
\text { (gall } \\
\text { min) }\end{array}$ & $\begin{array}{c}\text { Specific } \\
\text { capacity } \\
\text { [(gal/ } \\
\text { min)/ft] }\end{array}$ & $\begin{array}{l}\text { Source } \\
\text { of dis- } \\
\text { charge } \\
\text { data }\end{array}$ & $\begin{array}{l}\text { Type } \\
\text { of log } \\
\text { avall- } \\
\text { able }\end{array}$ & $\begin{array}{c}\text { Dale } \\
\text { water- } \\
\text { quality } \\
\text { parameter } \\
\text { moasured }\end{array}$ & $\begin{array}{c}\text { Specific } \\
\text { conduc- } \\
\text { tance } \\
(\mu S / \mathrm{cm})\end{array}$ & $\begin{array}{l}\text { pH } \\
\text { (otan- } \\
\text { dard } \\
\text { units) }\end{array}$ & $\begin{array}{l}\text { Tem- } \\
\text { por- } \\
\text { ature } \\
\left({ }^{\circ} \mathrm{C}\right)\end{array}$ & Well number \\
\hline \multicolumn{10}{|c|}{ Townsend Valley-Continued } \\
\hline 141.16 & 15 & .9 & D & D & $05-14-91$ & 563 & 7.9 & 9.0 & 09N02E34DAAA01 \\
\hline 94.45 & 4 & 1.3 & $\mathbf{S}$ & D & $05-13-91$ & 476 & 7.8 & 9.5 & 08N02E14BBBC01 \\
\hline 9.73 & 800 & 6.5 & D & D & .- & .. & -- & -- & 08N02E23CBBA01 \\
\hline 42.45 & 20 & .4 & D & D & -- & -- & -- & -- & 08N02E35BCDD01 \\
\hline 163.28 & 12 & .2 & D & $\mathbf{D}$ & $05-30-91$ & 492 & 7.8 & 10.5 & 07N01E03CDDD01 \\
\hline 164.67 & 5 & -. & D & D & -- & -- & -- & -- & 07N01E03CDDD02 \\
\hline 60.59 & 36 & 5.1 & D & - & -- & -- & -- & -- & 07N02E03ADDA01 \\
\hline 88.69 & 6 & .6 & D & D & $05-14-91$ & 613 & 7.6 & 10.5 & 07N02E15CBAB01 \\
\hline- & 30 & 5.0 & D & D & $10-21-92$ & 687 & 7.5 & 11.5 & 07N02E29CABC01 \\
\hline- & 440 & 9.6 & $\mathbf{D}$ & $\mathbf{D}$ & - & -- & -- & -- & 07N02E31ABAA01 \\
\hline - & 650 & 130 & D & D & -- & -- & .. & -- & 07NO2E31ADDA01 \\
\hline 7.77 & 600 & 38 & D & D & -- & -- & -- & -- & 07N02E31DBBA01 \\
\hline 185.80 & 20 & -- & D & D & $05-15-91$ & 476 & 7.8 & 11.5 & 07N02E34ABBD01 \\
\hline 87.13 & 10 & .2 & D & D & $05-15-91$ & 396 & 8.0 & 10.5 & 07N03E07BBBC01 \\
\hline 150.66 & 8 & -- & $\mathbf{D}$ & D & $05-30-91$ & 588 & 7.3 & 10.5 & 07N03E25DABB01 \\
\hline 23.85 & 10 & .1 & D & D & - & -- & -- & -- & 06N01E32DBBA01 \\
\hline 17.30 & -. & -- & - & - & $10-21-92$ & 478 & 7.7 & 11.0 & 06N02E09BACD01 \\
\hline-- & 1,000 & -- & D & D & -- & -- & -- & -- & 06N02E23ABAB01 \\
\hline 132.69 & -- & -- & - & D & -- & -- & -- & -- & 06N02E23DBCA01 \\
\hline-- & 1,200 & 21 & D & D & $08-09-91$ & 330 & 7.8 & 9.5 & 05N01E27BBCC01 \\
\hline 58.15 & 1,500 & 15 & D & D & -- & -- & -- & -- & 05N01E27BDA 01 \\
\hline 89.94 & 1,300 & 19 & D & D & -- & -- & -- & -. & 05N01E27CCBB01 \\
\hline 59.91 & 2,000 & 30 & D & D & -- & -- & -- & -- & 05N01E27CDAA01 \\
\hline 169.30 & 1,600 & 57 & D & D & -- & -- & -- & -- & 05N01E28BCAA01 \\
\hline 129.61 & 1,000 & 18 & D & D & - & -- & -- & -- & 05N01E33BACD01 \\
\hline 94.02 & 1,300 & 20 & D & D & -- & -- & -- & -- & 05N01E34CCCC01 \\
\hline 56.75 & -- & -- & - & - & -- & -- & -- & -- & 05N01E34DCCD01 \\
\hline 30.28 & 52 & -- & D & D & $10-19-92$ & 849 & 7.2 & 12.0 & 05N02E03ACDC01 \\
\hline-- & -- & -- & - & - & $10-21-92$ & 597 & 7.6 & 10.0 & 05N02E05BDCB01 \\
\hline 44.03 & 15 & 1.5 & D & D & -- & -- & -- & -- & 05N02E10ADDD01 \\
\hline 11.07 & 22 & 2.4 & D & $\mathbf{D}$ & $10-20-92$ & 1,020 & 7.4 & 10.0 & 05N02E22CBBA01 \\
\hline 41.85 & 6 & 4.2 & $\mathbf{s}$ & D & $10-19-92$ & 571 & 7.5 & 13.0 & 05N02E23DBCA01 \\
\hline$*+40$ & 60 & -- & D & D & 08-13-91 & 695 & 7.6 & 13.0 & 05N02E31DDCB01 \\
\hline-- & -- & -. & - & $\mathbf{D}$ & $10-20-92$ & 698 & 7.8 & 11.5 & 05N02E33DACA01 \\
\hline 65.15 & 1,500 & 38 & $\mathrm{D}$ & D & -- & -- & - & -- & 05N02E34AADD01 \\
\hline 38.98 & 2,000 & 26 & D & D & -- & -- & -- & -- & 04N01E02BBCC01 \\
\hline 56.58 & 150 & 1.7 & D & D & -- & -- & -- & -- & 04N01E02CCBB01 \\
\hline 59.25 & 2,200 & 27 & D & D & -- & -- & -- & -- & 04N01E02CCCB01 \\
\hline 79.80 & 50 & 10 & D & D & -- & -- & -- & -- & 04N01E03BABA01 \\
\hline 136.75 & 2,000 & 34 & $\mathrm{D}$ & D & -- & -- & -- & -. & 04N01E09ADB 01 \\
\hline
\end{tabular}


Table 1. Pecords of selected wells by basin, Northern Rocky Mountains intermontane basins, Montana (Continued)

\begin{tabular}{|c|c|c|c|c|c|c|c|c|c|}
\hline Well number & $\begin{array}{l}\text { Altitude } \\
\text { of lend } \\
\text { surface } \\
\text { (ft) }\end{array}$ & $\begin{array}{l}\text { Date well } \\
\text { constructed }\end{array}$ & $\begin{array}{c}\text { Geologic } \\
\text { unit }\end{array}$ & $\begin{array}{l}\text { Dopth } \\
\text { of well } \\
\text { (ft) }\end{array}$ & $\begin{array}{c}\text { Diam- } \\
\text { oler } \\
\text { of } \\
\text { casing } \\
\text { (in.) }\end{array}$ & $\begin{array}{l}\text { Type } \\
\text { of } \\
\text { finish }\end{array}$ & $\begin{array}{c}\text { Top of } \\
\text { open } \\
\text { inter- } \\
\text { val } \\
\text { (ft) }\end{array}$ & $\begin{array}{c}\text { Primary } \\
\text { uee of } \\
\text { water }\end{array}$ & $\begin{array}{c}\text { Date water } \\
\text { level } \\
\text { measured }\end{array}$ \\
\hline \multicolumn{10}{|c|}{ Townsend Valley-Continued } \\
\hline 04N01E10BBCB01 & 4,155 & $02-\quad-58$ & Ts & 447 & 7.0 & $\mathrm{o}$ & -- & $\mathbf{U}$ & $04-30-91$ \\
\hline 04N01E10BCCC01 & 4,150 & $12-08-58$ & Ts & 186 & 12.0 & $\mathbf{s}$ & 90 & I & $04-30-91$ \\
\hline 04N01E11BCCB01 & 4,095 & $08-08-64$ & Ts & 404 & 16.0 & $\mathbf{P}$ & 94 & I & $05-01-91$ \\
\hline 04N01E1ICBDD01 & 4,095 & 04- -77 & Ts & 202 & 16.0 & - & -- & I & $05-01-91$ \\
\hline 04N01E13BCBC01 & 4,085 & $02-09-77$ & Ts & 240 & 16.0 & $\mathbf{P}$ & 65 & $\mathbf{I}$ & $05-01-91$ \\
\hline 04N01E15BCBB01 & 4,185 & $05-29-67$ & Ts & 348 & 16.0 & $\mathbf{F}$ & 208 & I & 09-08-92 \\
\hline 04N01E15CDBA01 & 4,175 & $11-14-72$ & Ts & 342 & 16.0 & $\mathbf{F}$ & 212 & I & $05-01-91$ \\
\hline 04N01E19DDDD01 & 4,343 & 03-15-62 & Ts & 276 & 6.0 & $\mathbf{P}$ & 137 & $\mathbf{s}$ & $05-30-91$ \\
\hline 04N01E23BAC 01 & 4,127 & $06-27-64$ & Ts & 332 & 16.0 & $\mathbf{F}$ & 140 & I & $05-01-91$ \\
\hline 04N01E30DDC 01 & 4,315 & $04-15-63$ & Ts & 135 & 6.0 & $\mathbf{P}$ & 50 & $\mathbf{s}$ & $05-30-91$ \\
\hline \multicolumn{10}{|c|}{ Upper Blackfoot River Valley } \\
\hline 15N08W04CDAA01 & 5,360 & $11-07-63$ & Qg & 66 & 6.0 & $\mathbf{o}$ & - & $\mathbf{P}$ & $08-22-91$ \\
\hline 15N08W13ABCD01 & 5,030 & $05-18-86$ & Qal & 100 & 4.0 & $\mathbf{P}$ & 70 & $\mathbf{H}$ & $08-22-91$ \\
\hline $15 \mathrm{~N} 07 \mathrm{~W} 16 \mathrm{CCC} \quad 01$ & 5,139 & $08-06-70$ & Qg & 98 & 6.0 & $\mathbf{P}$ & 35 & $\mathbf{U}$ & $08-22-91$ \\
\hline 15N07W24AACD01 & 5,155 & -- & $\mathrm{Qg}$ & 46 & 9.0 & - & -- & $\mathbf{U}$ & $08-22-91$ \\
\hline $15 \mathrm{~N} 07 \mathrm{~W} 28 \mathrm{ABCB} 01$ & 5,022 & - & $\mathrm{Qg}$ & 121 & 6.0 & - & -- & $\mathbf{U}$ & $08-22-91$ \\
\hline 14N09W02CADD01 & 4,772 & 07-03-78 & QTsu & 110 & 6.0 & 0 & -- & $\mathbf{H}$ & $09-12-91$ \\
\hline 14N09W17ACBD01 & 4,725 & $07-21-89$ & Yms & 310 & 4.0 & $\mathbf{P}$ & 270 & $\mathbf{H}$ & $09-12-91$ \\
\hline 14N09W17ACDD01 & 4,580 & $06-25-88$ & Yms & 130 & 6.0 & $\mathbf{P}$ & 90 & $\mathbf{H}$ & $09-12-91$ \\
\hline 14N09W24DDD 01 & 4,549 & $-\quad-69$ & Qal & 48 & 6.0 & - & -- & $\mathbf{P}$ & $09-12-91$ \\
\hline 14N09W28ADDC01 & 4,465 & $08-01-91$ & QTsu & 55 & 6.0 & $\mathbf{O}$ & -- & $\mathbf{H}$ & $09-12-91$ \\
\hline 14N08W22BBAD01 & 4,658 & -- & Qal & 20 & 2.0 & - & -- & $\mathbf{H}$ & $09-12-91$ \\
\hline 14N07W05CAC 01 & 4,821 & 07-07-70 & Qal & 76 & 6.0 & $\mathbf{P}$ & 61 & $\mathrm{U}$ & 09-05-91 \\
\hline 14N07W05CDA 01 & 4,817 & 08-06-70 & Qal & 160 & 6.0 & $\mathbf{P}$ & 40 & $\mathbf{U}$ & $08-05-91$ \\
\hline 14N07W05CDD 02 & 4,814 & 08-06-70 & Qal & 200 & 6.0 & $\mathbf{P}$ & 60 & $\mathbf{U}$ & $09-05-91$ \\
\hline 13N08W06ADAB01 & 4,800 & $09-15-88$ & QTKe & 200 & 6.0 & $\mathbf{P}$ & 180 & $\mathbf{H}$ & $09-12-91$ \\
\hline \multicolumn{10}{|c|}{ Upper Clark Fork Valley } \\
\hline 11N12W31AACB01 & 3,980 & $08-11-70$ & Qal & 55 & 6.0 & $\mathbf{P}$ & 49 & $\mathbf{H}$ & $09-13-91$ \\
\hline 11N12W31CDBC01 & 4,020 & $07-20-75$ & Ts & 170 & 6.0 & $\mathbf{P}$ & 90 & $\mathbf{H}$ & $09-13-91$ \\
\hline 10N13W12BDDA01 & 4,120 & 05-23-80 & Ts & 137 & 6.0 & - & - & $\mathbf{H}$ & $09-13-91$ \\
\hline 10N13W22BDDA01 & 4,290 & $06-11-75$ & Ts & 85 & 6.0 & o & -- & $\mathbf{U}$ & $09-13-91$ \\
\hline 10N13W29DDAA01 & 4,360 & $04-27-73$ & Qal & 45 & 6.0 & $\mathbf{P}$ & 38 & $\mathbf{H}$ & $09-13-91$ \\
\hline 10N13W34DAAC01 & 4,245 & $-\quad-52$ & Qal & 50 & 6.0 & - & -- & $\mathbf{H}$ & $09-13-91$ \\
\hline 10N12W04AAAB01 & 4,120 & $09-11-78$ & Mzsh & 30 & 6.0 & 0 & - & $\mathbf{H}$ & $09-13-91$ \\
\hline 10N12W09CADA01 & 4,000 & $-\quad-44$ & Qal & 80 & 6.0 & - & - & $\mathbf{H}$ & $09-13-91$ \\
\hline 10N12W09CCCD01 & 4,020 & $07-03-74$ & Ts & 157 & 6.0 & 0 & - & $\mathbf{s}$ & $09-13-91$ \\
\hline 10N12W17BABB01 & 4,120 & $09-17-65$ & Mzsh & 227 & 6.0 & $\mathbf{P}$ & 145 & I & $09-13-91$ \\
\hline
\end{tabular}


Table 1. Records of selected wells by basin, Northern Rocky Mountains intermontane basins, Montana (Continued)

\begin{tabular}{|c|c|c|c|c|c|c|c|c|c|}
\hline $\begin{array}{c}\text { Water level } \\
\text { (fi) }\end{array}$ & $\begin{array}{c}\text { Dis- } \\
\text { charge } \\
\text { (gall } \\
\text { min) }\end{array}$ & $\begin{array}{c}\text { Specific } \\
\text { cepacity } \\
\text { [(gal/ } \\
\text { min)/ft] }\end{array}$ & $\begin{array}{c}\text { Source } \\
\text { of dis- } \\
\text { charge } \\
\text { data }\end{array}$ & $\begin{array}{l}\text { Type } \\
\text { of log } \\
\text { avali- } \\
\text { abla }\end{array}$ & $\begin{array}{c}\text { Date } \\
\text { water- } \\
\text { quallty } \\
\text { parameter } \\
\text { measured }\end{array}$ & $\begin{array}{c}\text { Specific } \\
\text { conduc- } \\
\text { tence } \\
(\mu S / \mathrm{cm})\end{array}$ & $\begin{array}{c}\text { pH } \\
\text { (sten- } \\
\text { dard } \\
\text { units) }\end{array}$ & $\begin{array}{l}\text { Tern- } \\
\text { per- } \\
\text { ature } \\
\left({ }^{\circ} \mathrm{C}\right)\end{array}$ & Well number \\
\hline
\end{tabular}

$\begin{array}{rrrl}117.35 & 5 & .2 & \text { D } \\ 113.45 & 1,500 & 39 & \text { D } \\ 65.30 & 2,400 & 12 & \text { D } \\ 57.08 & -. & -. & - \\ 40.17 & 1,400 & 13 & \text { D } \\ & & & \\ 144.52 & 2,000 & 20 & \text { D } \\ 135.52 & 2,000 & 22 & \text { D } \\ 107.47 & 8 & .1 & \text { D } \\ 78.14 & 3,400 & 42 & \text { D } \\ 32.03 & 6 & .4 & \text { D }\end{array}$

Townsend Valley-Continued

$\begin{array}{rccc}6.04 & 25 & 2.1 & \mathrm{D} \\ 75.48 & 20 & -- & \mathrm{D} \\ 26.39 & -- & -- & \\ 6.15 & -- & -- & -- \\ 5.93 & -- & & \\ & & & \\ 42.83 & 30 & 3.0 & \\ 175.60 & 15 & 3.0 & \mathrm{D} \\ 40.66 & 5 & .1 & \mathrm{D} \\ 6.06 & -- & -- & \\ 5.76 & 10 & 1.3 & \mathrm{D} \\ & & & - \\ 8.69 & -- & -- & - \\ 23.89 & -- & -- & - \\ 19.93 & -- & -- & - \\ 15.74 & -- & -- & -3\end{array}$

\section{Upper Blackfoot River Valley}

\begin{tabular}{|c|c|c|c|c|c|}
\hline D & $\cdots$ & - & - & $\cdots$ & 15N08W04CDAA01 \\
\hline D & -- & -- & -- & -- & 15N08W13ABCDO 1 \\
\hline - & -- & -- & -- & -- & $15 \mathrm{~N} 07 \mathrm{~W} 16 \mathrm{CCC}$ \\
\hline - & .. & -- & -- & -- & 15N07W24AACD01 \\
\hline - & -- & -- & - & -- & $15 \mathrm{~N} 07 \mathrm{~W} 28 \mathrm{ABCB}$ \\
\hline D & -- & - & -- & -- & 14N09W02CADI \\
\hline D & -- & -- & - & -- & 14N09W17ACBD \\
\hline D & - & -- & -- & -- & 14N09W17ACDD \\
\hline - & $10-07-74$ & -- & -- & 13.0 & 14N09W24DDD \\
\hline D & - & -- & -- & -. & 14N09W28ADDC \\
\hline- & -- & -- & $\cdots$ & -- & 14N08W22BBAD \\
\hline - & - & -- & -- & -- & 14N07W05CAC 0 \\
\hline - & -- & -- & -- & -. & 14N07W05CDA 0 \\
\hline - & -- & -- & -- & -- & 14N07W05CDD \\
\hline D & -- & -- & -- & -- & 13N08W06ADAB \\
\hline
\end{tabular}

\section{Upper Clark Fork Valley}

$\begin{array}{rccccccccc}39.29 & 40 & 2.7 & \text { D } & \text { D } & 10-29-85 & 740 & 7.2 & 10.0 & \text { IIN12W31AACB01 } \\ 50.87 & 10 & .7 & \text { D } & \text { D } & 06-10-87 & 2,190 & 7.0 & 10.0 & \text { IIN12W31CDBC01 } \\ 51.71 & 15 & .9 & \text { D } & \text { D } & 10-30-85 & 1,800 & 7.4 & 8.5 & \text { 10N13W12BDDA01 } \\ 46.34 & 20 & .8 & \text { D } & \text { D } & -- & -- & - & -- & \text { 10N13W22BDDA01 } \\ 4.69 & 15 & -- & \text { D } & \text { D } & 09-26-85 & 178 & 6.9 & 9.0 & \text { 10N13W29DDAA01 } \\ & & & & & & & & & \\ 4.15 & -- & -- & - & - & 09-26-85 & 595 & 7.3 & 8.0 & \text { 10N13W34DAAC01 } \\ 14.13 & 10 & -- & \text { D } & \text { D } & 10-28-85 & 603 & 7.4 & 8.5 & \text { 10N12W04AAAB01 } \\ 6.97 & 15 & -- & \text { O } & \text { D } & 10-30-85 & 611 & 7.3 & 9.5 & \text { 10N12W09CADA01 } \\ 29.74 & 15 & .2 & \text { D } & \text { D } & 09-24-85 & 732 & 7.3 & 9.5 & \text { 10N12W09CCD01 } \\ 102.99 & 70 & 10 & \text { D } & \text { D } & 10-04-85 & 1,540 & 7.0 & 9.0 & \text { 10N12W17BABB01 }\end{array}$


Table 1. Records of selected wells by basin, Northern Rocky Mountains intermontane basins, Montana (Continued)

\begin{tabular}{|c|c|c|c|c|c|c|c|c|c|}
\hline Well number & $\begin{array}{l}\text { Alttude } \\
\text { of land } \\
\text { surface } \\
\text { (ft) }\end{array}$ & $\begin{array}{l}\text { Date well } \\
\text { constructed }\end{array}$ & $\begin{array}{c}\text { Geologlc } \\
\text { unit }\end{array}$ & $\begin{array}{c}\text { Depth } \\
\text { of well } \\
\text { (tt) }\end{array}$ & $\begin{array}{c}\text { Diam- } \\
\text { oter } \\
\text { of } \\
\text { casing } \\
\text { (ln.) }\end{array}$ & $\begin{array}{l}\text { Type } \\
\text { of } \\
\text { finish }\end{array}$ & $\begin{array}{l}\text { Top of } \\
\text { open } \\
\text { Inter- } \\
\text { val } \\
\text { (ft) }\end{array}$ & $\begin{array}{l}\text { Primary } \\
\text { use of } \\
\text { water }\end{array}$ & $\begin{array}{c}\text { Date water } \\
\text { level } \\
\text { measured }\end{array}$ \\
\hline \multicolumn{10}{|c|}{ Upper Clark Fork Valley-Continued } \\
\hline 10N12W30BABD01 & 4,120 & $09-06-72$ & Ts & 36 & 6.0 & $\mathbf{P}$ & 30 & $\mathbf{H}$ & $09-13-91$ \\
\hline 10NIIW20CACA0I & 4,134 & -- & QTsu & 70 & 6.0 & - & -- & $\mathbf{H}$ & $09-13-91$ \\
\hline 10N1IW20CADA01 & 4,134 & -- & QTsu & 225 & 6.0 & - & -- & $\mathbf{H}$ & $09-13-91$ \\
\hline 10N1IW25CCDD01 & 4,190 & -. & Ts & -- & 6.0 & - & -- & $\mathbf{H}$ & $09-13-91$ \\
\hline 10N11W36BCAD01 & 4,220 & $07-12-68$ & Ts & 100 & 6.0 & $\mathbf{P}$ & 47 & $\mathbf{P}$ & $09-13-91$ \\
\hline I0N10W19DCCC01 & 4,380 & $02-10-76$ & Mzsh & 40 & 6.0 & $\mathbf{P}$ & 30 & $\mathbf{H}$ & $09-13-91$ \\
\hline I0N10W31BABA02 & 4,200 & $06-22-83$ & Qal & 63 & 6.6 & $\mathbf{P}$ & 44 & $\mathbf{P}$ & $09-13-91$ \\
\hline 09N13W02CBBC01 & 4,250 & $09-30-71$ & Qal & 35 & 6.0 & $\mathbf{P}$ & 30 & H & $09-13-91$ \\
\hline 09N13W10DCCC01 & 4,330 & $12-\quad-68$ & Qal & 23 & 6.0 & $\mathbf{O}$ & -- & $\mathbf{H}$ & $09-13-91$ \\
\hline 09N13W22BCDA01 & 4,440 & $07-05-74$ & Qal & 38 & 6.0 & $\mathrm{O}$ & -- & $\mathbf{H}$ & $09-13-91$ \\
\hline 09N11W01BCAC01 & 4,365 & $05-29-80$ & Ts & 81 & 6.0 & 0 & -- & $\mathbf{H}$ & $09-13-91$ \\
\hline 09N10W04BDBC01 & 4,330 & $06-20-72$ & Mzsh & 93 & 6.0 & $\mathbf{P}$ & 45 & $\mathbf{H}$ & $09-12-91$ \\
\hline 09N10W 10CDBD01 & 4,290 & $03-21-75$ & Mzsh & 118 & 6.0 & $\mathbf{P}$ & 78 & $\mathbf{H}$ & $09-12-91$ \\
\hline 09N10W 10CDCC01 & 4,275 & $06-20-73$ & Mzsh & 50 & 6.0 & $\mathbf{P}$ & 31 & $\mathbf{S}$ & $09-12-91$ \\
\hline 09N10W15AABB01 & 4,560 & $01-28-77$ & Mzsh & 280 & 6.0 & $\mathbf{P}$ & 240 & $\mathbf{H}$ & $09-12-91$ \\
\hline 09N10W23ACBD01 & 4,335 & $08-11-75$ & QTKe & 100 & 6.0 & $\mathbf{P}$ & 80 & $\mathbf{H}$ & $09-12-91$ \\
\hline 09N10W24BBCA01 & 4,370 & $04-09-74$ & QTKe & 85 & 6.0 & $\mathbf{P}$ & 47 & $\mathbf{P}$ & $09-12-91$ \\
\hline 09N09W28CABD01 & 4,570 & $06-07-77$ & Mzsh & 220 & 6.0 & $\mathbf{P}$ & 160 & $\mathbf{H}$ & $09-12-91$ \\
\hline 09N09W28CCDD01 & 4,380 & $06-05-86$ & Qal & 17 & 2.0 & $\mathbf{s}$ & 7 & $\mathbf{U}$ & $09-12-91$ \\
\hline 09N09W32BACA01 & 4,419 & $11-30-78$ & Ts & 57 & 6.0 & $\mathbf{P}$ & 30 & $\mathbf{S}$ & $09-12-91$ \\
\hline 09N09W33CDBC01 & 4,460 & $12-11-78$ & Ts & 127 & 6.0 & $\mathbf{P}$ & 80 & $\mathbf{s}$ & $09-12-91$ \\
\hline 08NIOW23DBAD01 & 5,080 & $11-09-84$ & Ts & 115 & 6.0 & $\mathrm{O}$ & -- & $\mathbf{s}$ & $09-12-91$ \\
\hline 08N09W11DBA 01 & 4,630 & $06-\quad-76$ & QTKe & 300 & 14.0 & $\mathbf{P}$ & 40 & $\mathrm{U}$ & $09-12-91$ \\
\hline 08N09W14DADA01 & 4,600 & $08-03-72$ & Ts & 74 & 6.0 & $\mathbf{P}$ & 55 & $\mathbf{S}$ & $09-12-91$ \\
\hline 08N09W20CAAB01 & 4,562 & $02-20-79$ & Ts & 117 & 6.0 & $\mathbf{P}$ & 60 & $\mathbf{s}$ & $09-12-91$ \\
\hline 08N09W2IDDAB01 & 4,505 & $07-21-77$ & Ts & 77 & 6.0 & $\mathbf{P}$ & 70 & H & $09-12-91$ \\
\hline 08N09W25DCBB0I & 4,742 & $06-01-77$ & Qal & 29 & 6.0 & $\mathbf{P}$ & 22 & $\mathbf{s}$ & $09-12-91$ \\
\hline 08N09W27BDDD01 & 4,565 & $08-12-82$ & Ts & 95 & 6.0 & $\mathbf{P}$ & 70 & $\mathbf{U}$ & $08-16-91$ \\
\hline 08N09W32AADD01 & 4,490 & $06-10-86$ & Qal & 9 & 2.0 & $\mathbf{S}$ & 9 & $\mathbf{U}$ & $09-12-91$ \\
\hline 08N09W32AADD02 & 4,490 & $06-\quad-87$ & Ts & 59 & 2.0 & $\mathbf{S}$ & 49 & $\mathbf{U}$ & $09-12-91$ \\
\hline 07N10W03ABBD01 & 5,018 & $-\quad-56$ & Ts & 220 & 30.0 & $\mathbf{G}$ & 170 & H & $09-12-91$ \\
\hline 07N10W15DBCB01 & 5,055 & $09--61$ & Ts & 75 & 6.0 & - & - & $\mathbf{U}$ & $09-12-91$ \\
\hline 07N09W03CABBOI & 4,610 & $09-12-75$ & Ts & 150 & 6.0 & $\mathbf{P}$ & 132 & $\mathbf{H}$ & $09-12-91$ \\
\hline 07N09W04DDCA01 & 4,535 & $-\quad-79$ & QTsu & 115 & 6.0 & - & - & $\mathbf{H}$ & $09-13-91$ \\
\hline 07N09W08ADD 01 & 4,551 & $-\quad-57$ & Qal & 13 & 36.0 & $\mathrm{O}$ & - & $\mathbf{U}$ & $09-18-91$ \\
\hline 07N09W1ICDBBOI & 4,740 & 04-20-79 & Ts & 30 & 6.0 & $\mathbf{P}$ & 23 & $\mathbf{S}$ & $09-12-91$ \\
\hline 07N09W16AADA01 & 4,585 & $05-30-75$ & Ts & 89 & 6.6 & $\mathbf{P}$ & 82 & $\mathbf{H}$ & $09-12-91$ \\
\hline 07N09W1 6AADB01 & 4,580 & -- & Ts & 105 & 6.0 & - & -- & $\mathbf{H}$ & $09-12-91$ \\
\hline 07N09W20CADD01 & 4,620 & $02-\quad-52$ & Ts & 180 & 8.0 & $\mathbf{P}$ & 83 & $\mathbf{N}$ & $09-12-91$ \\
\hline 07N09W27CCDD01 & 4,760 & $02-05-80$ & Ts & 246 & 6.0 & $\mathbf{P}$ & 220 & $\mathbf{s}$ & $09-12-91$ \\
\hline
\end{tabular}


Table 1. Records of selected wells by basin, Northern Rocky Mountains intermontane basins, Montana (Continued)

\begin{tabular}{|c|c|c|c|c|c|c|c|c|c|}
\hline $\begin{array}{l}\text { Water lovel } \\
\text { (ft) }\end{array}$ & $\begin{array}{l}\text { Dis } \\
\text { charge } \\
\text { (gall } \\
\text { min) }\end{array}$ & $\begin{array}{l}\text { Specific } \\
\text { capacity } \\
\text { [(gal/ } \\
\text { min)/ft] }\end{array}$ & $\begin{array}{l}\text { Source } \\
\text { of dis- } \\
\text { charge } \\
\text { data }\end{array}$ & $\begin{array}{l}\text { Type } \\
\text { cf log } \\
\text { avall- } \\
\text { able }\end{array}$ & $\begin{array}{c}\text { Date } \\
\text { water- } \\
\text { quallty } \\
\text { parameter } \\
\text { measured }\end{array}$ & $\begin{array}{l}\text { Specific } \\
\text { conduc- } \\
\text { tanca } \\
(\mu S / c m)\end{array}$ & $\begin{array}{l}\text { pH } \\
\text { (stan- } \\
\text { dard } \\
\text { Units) }\end{array}$ & $\begin{array}{l}\text { Tem- } \\
\text { per- } \\
\text { ature } \\
\left({ }^{\circ} \mathrm{C}\right)\end{array}$ & Woll number \\
\hline \multicolumn{10}{|c|}{ Upper Clark Fork Valley-Continued } \\
\hline 11.36 & 30 & 1.7 & D & D & $10-30-85$ & 820 & 7.2 & 9.5 & 10N12W30BABD01 \\
\hline 9.30 & -- & -- & - & - & $04-29-87$ & 359 & 8.0 & 7.0 & 10N11W20CACA01 \\
\hline 10.00 & -- & -- & - & - & $04-29-87$ & 342 & 8.6 & 8.5 & 10N11W20CADA01 \\
\hline 13.70 & -- & -- & - & - & $11-05-85$ & 615 & 8.8 & 8.0 & 10N11W25CCDD01 \\
\hline 16.47 & 12 & .2 & D & $\mathbf{D}$ & $10-16-85$ & 739 & 8.1 & 11.0 & 10N11W36BCAD01 \\
\hline 24.88 & 30 & -- & D & D & $11-06-85$ & 810 & 7.2 & 9.0 & 10N10W19DCCC01 \\
\hline 5.78 & 37 & 4.9 & D & D & $10-09-86$ & 610 & 7.4 & 12.0 & 10N10W31BABA02 \\
\hline 12.92 & 15 & -- & D & D & $10-04-85$ & 409 & 7.5 & 10.5 & 09N13W02CBBC01 \\
\hline 8.45 & 50 & 3.9 & D & D & $09-26-85$ & 400 & 7.4 & 12.5 & 09N13W10DCCCOI \\
\hline 10.68 & 25 & 1.7 & D & $\mathrm{D}$ & $09-26-85$ & 403 & 7.4 & 9.5 & 09N13W22BCDA01 \\
\hline 30.27 & 22 & 1.3 & $\mathbf{D}$ & $\mathrm{D}$ & $11-06-85$ & 710 & 7.5 & 8.5 & 09NIIW01BCAC01 \\
\hline 31.60 & 15 & 1.5 & D & $\mathbf{D}$ & $07-17-85$ & 655 & 7.6 & 9.0 & 09N10W04BDBC01 \\
\hline 19.68 & 10 & .1 & D & D & $07-17-85$ & 1,100 & 7.6 & 10.0 & 09N10W10CDBD01 \\
\hline 5.81 & 10 & .3 & D & D & -- & -- & -- & -. & 09N10W10CDCC01 \\
\hline 220.20 & 30 & .5 & D & $\mathrm{D}$ & $05-28-87$ & 1,120 & 8.1 & 12.0 & 09N10W15AABB01 \\
\hline 7.76 & 50 & 2.5 & $\mathrm{D}$ & D & -. & -. & -. & -- & 09N10W23ACBD01 \\
\hline 32.50 & 20 & .4 & D & D & $11-05-85$ & 665 & 7.3 & 9.0 & 09N10W24BBCA01 \\
\hline 67.32 & -- & -. & - & D & $05-06-87$ & 755 & 7.2 & 12.0 & 09N09W28CABD01 \\
\hline 4.90 & -. & -- & - & $\mathrm{D}$ & -- & -- & -- & -. & 09N09W28CCDD01 \\
\hline 10.40 & 20 & .5 & D & D & 09-05-85 & 629 & 7.3 & 8.0 & 09N09W32BACA01 \\
\hline 27.29 & 20 & .2 & D & D & $10-24-85$ & 621 & 7.4 & 7.0 & 09N09W33CDBC01 \\
\hline 25.29 & 30 & .4 & D & D & -- & -- & -- & -- & 08N10W23DBAD01 \\
\hline 20.37 & 750 & 13 & D & D & -- & -- & -- & -- & 08N09W11DBA 01 \\
\hline 30.15 & 15 & .3 & D & D & $08-07-83$ & 760 & 7.4 & 8.5 & 08N09W14DADA01 \\
\hline 44.66 & 20 & .3 & D & D & - & $\cdots$ & - & -- & 08N09W20CAAB01 \\
\hline 17.47 & 40 & -- & $\mathrm{D}$ & D & $05-05-87$ & 380 & 6.7 & 10.0 & 08N09W21DDAB01 \\
\hline 5.10 & 20 & -. & D & D & $07-31-85$ & 420 & 7.3 & 7.5 & 08N09W25DCBB01 \\
\hline 24.81 & 15 & -- & D & D & - & -- & - & -- & 08N09W27BDDD01 \\
\hline 3.01 & -- & -- & - & D & - & $\cdots$ & -- & .- & 08N09W32AADD01 \\
\hline 3.18 & -- & -- & - & - & $07-14-87$ & 330 & 7.2 & 12.0 & 08N09W32AADD02 \\
\hline 29.60 & 200 & 2.1 & D & D & $10-23-85$ & 421 & 7.6 & 7.5 & 07N10W03ABBD01 \\
\hline 16.62 & -- & -- & - & D & -- & -- & -- & -- & 07N10W15DBCB01 \\
\hline 69.85 & 14 & -. & D & D & $08-16-85$ & 238 & 7.2 & 10.5 & $07 \mathrm{~N} 09 \mathrm{~W} 03 \mathrm{CABB} 01$ \\
\hline 22.50 & -- & -- & - & - & 04-29-87 & 438 & 6.3 & 12.0 & 07N09W04DDCA01 \\
\hline 9.59 & -- & -- & - & - & -- & -. & -- & -- & 07N09W08ADD 01 \\
\hline 18.67 & 25 & -- & D & D & $10-23-85$ & 1,050 & 7.1 & 9.0 & 07N09W11CDBB01 \\
\hline 19.40 & 20 & -- & D & D & $04-28-87$ & 247 & 6.8 & 12.0 & 07N09W16AADA01 \\
\hline 16.97 & -. & -- & - & - & $04-27-87$ & 254 & 6.7 & 12.0 & 07N09W16AADB01 \\
\hline 37.98 & 120 & 13 & D & D & $08-16-85$ & 555 & 7.4 & 10.5 & 07N09W20CADD01 \\
\hline 56.17 & 5 & .. & D & $\mathbf{D}$ & -. & -- & -- & -- & 07N09W27CCDD01 \\
\hline
\end{tabular}


Table 1. Records of selected wells by basin, Northern Rocky Mountains intermontane basins, Montana (Continued)

\begin{tabular}{|c|c|c|c|c|c|c|c|c|c|}
\hline Well number & $\begin{array}{l}\text { Altitude } \\
\text { of land } \\
\text { surfece } \\
\text { (ft) }\end{array}$ & $\begin{array}{l}\text { Date well } \\
\text { constructed }\end{array}$ & $\begin{array}{c}\text { Goologle } \\
\text { unit }\end{array}$ & $\begin{array}{c}\text { Depth } \\
\text { of woll } \\
\text { (ft) }\end{array}$ & $\begin{array}{l}\text { Dlam- } \\
\text { eter } \\
\text { of } \\
\text { casing } \\
\text { (in.) }\end{array}$ & $\begin{array}{l}\text { Type } \\
\text { of } \\
\text { finieh }\end{array}$ & $\begin{array}{l}\text { Top of } \\
\text { open } \\
\text { Inter- } \\
\text { val } \\
\text { (tt) }\end{array}$ & $\begin{array}{c}\text { Primary } \\
\text { uee of } \\
\text { weter }\end{array}$ & $\begin{array}{c}\text { Date water } \\
\text { lovel } \\
\text { meacured }\end{array}$ \\
\hline \multicolumn{10}{|c|}{ Upper Clerk Fork Valley_Continued } \\
\hline 07N09W31CCAD01 & 4,747 & $10-01-81$ & Ts & 205 & 8.0 & $\mathbf{P}$ & 160 & $\mathbf{U}$ & $09-12-91$ \\
\hline onN09W31CCBB01 & 4,760 & $08-05-71$ & Ts & 150 & 6.0 & $\mathbf{P}$ & 144 & $\mathbf{H}$ & $09-12-91$ \\
\hline $06 \mathrm{~N} 10 \mathrm{~W} 13 \mathrm{BCBB} 01$ & 4,820 & $10-27-72$ & Ts & 127 & 6.0 & $\mathbf{P}$ & 120 & $\mathbf{H}$ & $09-12-91$ \\
\hline 06N10W23DCDD01 & 4,900 & $11-05-85$ & Ts & 112 & 6.0 & $\mathbf{P}$ & 58 & $\mathbf{H}$ & $09-13-91$ \\
\hline $06 \mathrm{~N} 10 \mathrm{~W} 27 \mathrm{CCCCO}$ & 5,005 & -- & Ts & 89 & 6.0 & - & - & $\mathbf{U}$ & $09-13-91$ \\
\hline 06N10W28BBBB01 & 5,105 & $09-28-73$ & QTsu & 80 & 6.0 & $\mathbf{P}$ & 75 & $\mathbf{H}$ & $09-12-91$ \\
\hline 06N09W04DDAC01 & 4,650 & $-\quad-84$ & QTsu & 70 & -. & - & - & $\mathbf{H}$ & $10-17-91$ \\
\hline 06N09W05DDAA01 & 4,665 & .- & QTsu & 80 & -- & - & -- & H & 09-12-91 \\
\hline 06N09W07BCCC01 & 4,770 & $08-01-77$ & Ts & 436 & 6.0 & $\mathbf{P}$ & 230 & $\mathbf{U}$ & $09-12-91$ \\
\hline 06N09W09DADD01 & 4,690 & $07-24-85$ & Ts & 80 & 6.0 & $\mathbf{P}$ & 70 & $\mathbf{H}$ & $10-17-91$ \\
\hline 06N09W10BBA 01 & 4,720 & $04-17-79$ & Ts & 135 & 6.0 & $\mathbf{P}$ & 113 & $\mathbf{H}$ & $09-12-91$ \\
\hline $06 \mathrm{~N} 09 \mathrm{~W} 21 \mathrm{CDBC01}$ & 4,785 & -60 & Ts & 150 & 6.0 & - & - & - & $09-18-91$ \\
\hline 06N09W29DAAD01 & 4,725 & $02-13-87$ & Qal & 60 & 6.6 & $\mathbf{P}$ & 52 & $\mathbf{s}$ & $09-13-91$ \\
\hline 05N10W03BBBB01 & 5,033 & $08-24-73$ & Ts & 149 & 6.0 & $\mathbf{P}$ & 142 & $\mathbf{H}$ & $09-13-91$ \\
\hline $05 \mathrm{~N} 10 \mathrm{~W} 10 \mathrm{CCBC} 01$ & 4,965 & $08-31-73$ & Ts & 115 & 6.0 & $\mathbf{P}$ & 106 & $\mathbf{S}$ & $09-13-91$ \\
\hline 05N10W20DCCA01 & 5,003 & -- & Qal & 50 & 6.0 & - & - & $\mathbf{H}$ & $09-13-91$ \\
\hline 05N09W05CDBB01 & 4,780 & $12-01-78$ & Qal & 31 & 6.0 & $\mathbf{P}$ & 24 & $\mathbf{H}$ & $09-13-91$ \\
\hline 05N09W05CDDD0I & 4,845 & $-\quad-84$ & QTsu & 115 & 6.0 & - & -- & $\mathbf{H}$ & $09-13-91$ \\
\hline 05N09W17BCCA01 & 4,830 & --31 & QTsu & 139 & 6.0 & - & -- & $\mathbf{H}$ & $09-13-91$ \\
\hline 05N09W17CACA01 & 4,870 & $07-06-76$ & Ts & 115 & 6.0 & $\mathbf{P}$ & 109 & $\mathbf{S}$ & $09-13-91$ \\
\hline 04NIIW0IBCCD01 & 5,190 & -- & Qal & 72 & 8.0 & - & - & $\mathrm{u}$ & $09-13-91$ \\
\hline 04N11W01CAA 01 & 5,167 & $09-25-73$ & Qal & 115 & 6.0 & $\mathbf{P}$ & 60 & $\mathrm{U}$ & $09-13-91$ \\
\hline 04N11W08ADBB01 & 5,790 & $05-19-81$ & Ts & 120 & 6.0 & $\mathrm{O}$ & - & $\mathrm{C}$ & $10-17-91$ \\
\hline 04N10W05AACCOI & 5,047 & 06- -37 & Qal & 34 & 6.0 & - & -- & $\mathbf{U}$ & $09-13-91$ \\
\hline 04N10W05AACCO2 & 5,045 & $06-\quad-37$ & Qal & 98 & 6.0 & - & - & $\mathrm{U}$ & $09-13-91$ \\
\hline 04N10W06BADD01 & 5,105 & $10--77$ & Qal & 77 & 6.0 & $\mathbf{P}$ & 70 & $\mathrm{U}$ & $09-13-91$ \\
\hline 04NIOW1ODADA02 & 4,950 & $10-20-88$ & Qal & 44 & 6.0 & $\mathbf{P}$ & 38 & $\mathbf{H}$ & $10-17-9 \mid$ \\
\hline 04N10WIODC $\quad 02$ & 4,978 & -60 & Qal & 20 & 4.0 & 0 & - & $\mathbf{F}$ & $10-03-91$ \\
\hline 04NIOWIICBAC01 & 4,940 & $10-27-78$ & Qal & 169 & 6.0 & $\mathrm{O}$ & - & $\mathbf{H}$ & $10-17-91$ \\
\hline \multicolumn{10}{|c|}{ Upper Ruby Valley } \\
\hline 07S04W31DCB 01 & 5,475 & $04-07-72$ & Ts & 117 & 6.0 & 0 & - & $\mathbf{H}$ & $06-24-91$ \\
\hline 08S04W06ABBA01 & 5,510 & $09-06-74$ & Ts & 95 & 6.0 & $\mathbf{P}$ & 85 & $\mathrm{U}$ & $06-24-91$ \\
\hline 08504W34BCC 01 & 5,715 & $07-30-76$ & Ts & 55 & 6.0 & $\mathbf{P}$ & 41 & $\mathbf{H}$ & $06-23-91$ \\
\hline 09S05W I 2CCDD01 & 6,209 & -23 & Ts & 250 & 6.0 & - & - & $\mathbf{s}$ & 06-24-91 \\
\hline 09S04W01DCDD01 & 5,860 & $07-15-89$ & Ts & 113 & 6.0 & $\mathbf{P}$ & 106 & $\mathbf{H}$ & $06-23-91$ \\
\hline 09S04W01DCDD02 & 5,860 & -.59 & Ts & 52 & 6.0 & - & .. & $\mathbf{U}$ & $06-23-91$ \\
\hline 09S04W11BAAD01 & 5,810 & 05-03-78 & Ts & 51 & 6.0 & $\mathbf{P}$ & 44 & $\mathbf{H}$ & 06-23-91 \\
\hline
\end{tabular}


Table 1. Records of selected wells by basin, Northern Rocky Mountains intermontane basins, Montana (Continued)

\begin{tabular}{|c|c|c|c|c|c|c|c|c|c|}
\hline $\begin{array}{c}\text { Water level } \\
\text { (ft) }\end{array}$ & $\begin{array}{c}\text { Dis- } \\
\text { charge } \\
\text { (gal/ } \\
\text { min) }\end{array}$ & $\begin{array}{c}\text { Specific } \\
\text { capacity } \\
\text { [(gal/ } \\
\text { min }) / f t]\end{array}$ & $\begin{array}{c}\text { Source } \\
\text { of dis- } \\
\text { charge } \\
\text { data }\end{array}$ & $\begin{array}{l}\text { Type } \\
\text { of log } \\
\text { avail- } \\
\text { able }\end{array}$ & $\begin{array}{c}\text { Dato } \\
\text { water- } \\
\text { quality } \\
\text { parameter } \\
\text { moaeured }\end{array}$ & $\begin{array}{l}\text { Specific } \\
\text { conduc- } \\
\text { tance } \\
(\mu S / c m)\end{array}$ & $\begin{array}{l}\text { pH } \\
\text { (stan- } \\
\text { dard } \\
\text { unita) }\end{array}$ & $\begin{array}{l}\text { Tom- } \\
\text { per- } \\
\text { ature } \\
\left({ }^{\circ} \mathrm{C}\right)\end{array}$ & Well number \\
\hline
\end{tabular}

\begin{tabular}{|c|c|c|c|c|c|c|c|c|c|}
\hline 104.76 & 80 & 2.7 & $\mathrm{D}$ & D & -- & -. & -- & -- & 07N09W31CCAD01 \\
\hline 107.94 & 15 & -- & $\mathrm{D}$ & D & $10-23-85$ & 354 & 7.8 & 10.0 & OTN09W31CCBB01 \\
\hline 107.66 & 15 & -- & D & D & $08-15-85$ & 420 & 7.1 & 9.5 & $06 \mathrm{~N} 10 \mathrm{~W} 13 \mathrm{BCBB} 01$ \\
\hline 86.17 & 18 & -- & $\mathrm{D}$ & D & $10-25-85$ & 480 & 7.6 & 9.0 & $06 \mathrm{~N} 10 \mathrm{~W} 23 \mathrm{DCDD} 01$ \\
\hline 48.76 & -- & -- & - & - & -- & -- & -- & - & $06 \mathrm{~N} 10 \mathrm{~W} 27 \mathrm{CCCC} 01$ \\
\hline 58.34 & 10 & -. & D & D & - & -. & -- & .. & 06N10W28BBBB01 \\
\hline 18.25 & -- & -- & - & - & $05-29-87$ & 282 & 7.3 & 12.5 & 06N09W04DDAC01 \\
\hline 12.62 & .. & -. & - & - & $04-22-87$ & 261 & 7.2 & 12.0 & 06N09W05DDAA01 \\
\hline 47.48 & 2,400 & 17 & D & D & -- & -- & ـ- & -- & 06N09W07BCCC01 \\
\hline 41.93 & 10 & -- & $\mathrm{D}$ & D & $04-23-87$ & 277 & 7.0 & 15.0 & 06N09W09DADD01 \\
\hline 87.83 & 20 & -. & $\mathrm{D}$ & D & $08-07-85$ & 383 & 7.2 & 9.5 & 06N09W10BBA 01 \\
\hline 92.54 & - & -- & - & - & -- & -- & -- & -- & 06N09W21 CDBC01 \\
\hline 13.15 & 15 & .8 & $\mathrm{D}$ & $\mathrm{D}$ & $04-20-87$ & 406 & 6.3 & 10.0 & 06N09W29DAAD01 \\
\hline 105.08 & 15 & .6 & D & D & 09-04-85 & 510 & 7.2 & 10.5 & 05N10W03BBBB01 \\
\hline 73.60 & 15 & -- & $\mathrm{D}$ & D & -- & -. & -. & -- & $05 \mathrm{~N} 10 \mathrm{~W} 10 \mathrm{CCBC} 01$ \\
\hline 20.34 & -. & -. & - & - & $10-24-85$ & 388 & 7.9 & 8.5 & 05N10W20DCCA01 \\
\hline 15.86 & 15 & - & D & D & $07-25-85$ & 418 & 7.3 & 10.0 & 05N09W05CDBB01 \\
\hline 76.95 & -- & -- & - & - & $07-25-85$ & 327 & 7.2 & 10.5 & 05N09W05CDDD01 \\
\hline 45.13 & -- & .. & - & - & $04-20-87$ & 308 & 7.2 & 11.5 & 05N09W17BCCA01 \\
\hline 91.76 & 13 & -- & D & D & 08-20-85 & 395 & 7.1 & 10.0 & 05N09W17CACA01 \\
\hline 30.52 & -- & -- & - & - & -- & -- & -- & -- & 04N11W01BCCD01 \\
\hline 35.71 & -- & -- & - & D & -. & -. & -- & -- & 04N11W01CAAA01 \\
\hline 28.83 & -- & -. & - & D & $08-21-85$ & 104 & 6.9 & 7.0 & 04N11W08ADBB01 \\
\hline 29.53 & -. & -- & - & - & -- & -- & -- & -- & 04N10W05AACC01 \\
\hline 28.11 & -- & -- & - & - & -- & -. & - & -. & 04N10W05AACC02 \\
\hline 65.68 & -- & -- & - & D & -- & -- & -- & -- & 04N10W06BADD01 \\
\hline 6.16 & 12 & -- & D & D & -- & -- & -. & $\cdots$ & 04N10W10DADA02 \\
\hline 4.69 & -- & -- & - & - & -- & -- & -- & -- & 04N10W10DC $\quad 02$ \\
\hline 4.21 & 50 & 3.1 & $\mathbf{D}$ & $\mathrm{D}$ & $08-21-85$ & 232 & 7.2 & 10.0 & 04N10W11CBAC01 \\
\hline \multicolumn{10}{|c|}{ Upper Ruby Valley } \\
\hline 40.47 & 12 & .9 & $\mathrm{D}$ & D & $06-24-91$ & 779 & 7.7 & 12.0 & 07S04W31DCB 01 \\
\hline 64.32 & 20 & 4.0 & D & D & -- & -- & -- & -. & 08S04W06ABBA01 \\
\hline 6.18 & 45 & -- & $\mathrm{D}$ & $\mathbf{D}$ & $06-23-91$ & 502 & 7.8 & 7.5 & 08S04W34BCC 01 \\
\hline 192.65 & -- & -- & - & - & -- & - & -- & -- & 09S05W12CCDD01 \\
\hline 38.89 & 16 & -- & D & $\mathbf{D}$ & -- & -- & -- & -- & 09S04W01DCDD01 \\
\hline 30.96 & - & -- & - & - & -- & $\ddot{s e r}$ & - & - & 09S04W01DCDD02 \\
\hline 7.96 & 18 & 1.0 & $\mathrm{D}$ & $\mathrm{D}$ & $06-23-91$ & 595 & 7.5 & 13.0 & 09S04W11BAAD01 \\
\hline
\end{tabular}


Table 1. Records of selected wells by basin, Northern Rocky Mountains intermontane basins, Montana (Continued)

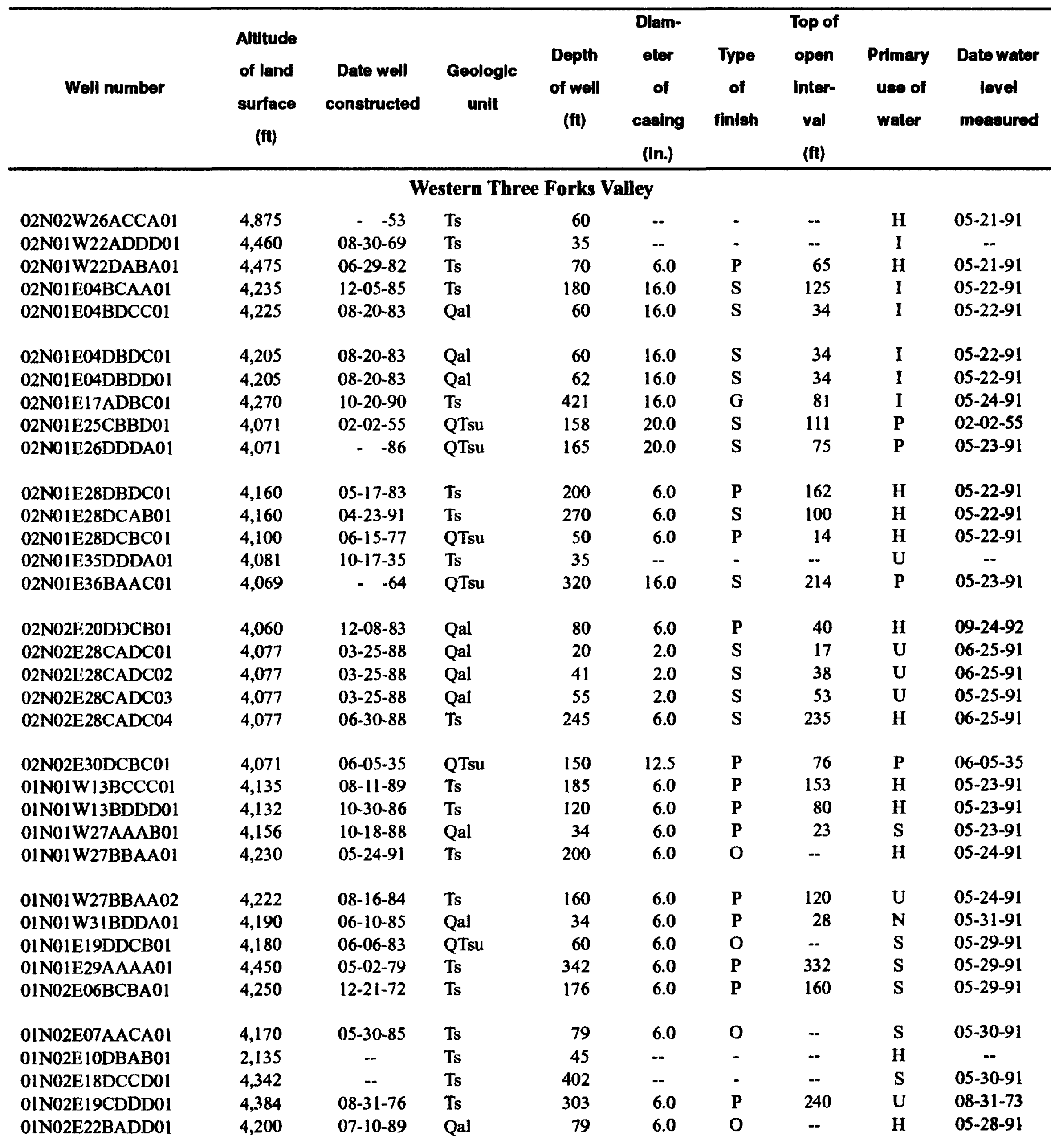


Table 1. Records of selected wells by basin, Northern Rocky Mountains intermontane basins, Montana (Continued)

\begin{tabular}{|c|c|c|c|c|c|c|c|c|c|}
\hline $\begin{array}{c}\text { Water level } \\
\text { (ft) }\end{array}$ & $\begin{array}{c}\text { Dis- } \\
\text { charge } \\
\text { (gal/ } \\
\text { min) }\end{array}$ & $\begin{array}{c}\text { Specific } \\
\text { capecity } \\
\text { [(gal/ } \\
\text { min)/ft] }\end{array}$ & $\begin{array}{c}\text { Source } \\
\text { of dle- } \\
\text { charge } \\
\text { dala }\end{array}$ & $\begin{array}{l}\text { Type } \\
\text { of log } \\
\text { avall- } \\
\text { ablo }\end{array}$ & $\begin{array}{c}\text { Date } \\
\text { water- } \\
\text { quality } \\
\text { paramelor } \\
\text { measured }\end{array}$ & $\begin{array}{l}\text { Speclfic } \\
\text { conduc- } \\
\text { tanca } \\
(\mu S / c m)\end{array}$ & $\begin{array}{l}\text { pH } \\
\text { (stan- } \\
\text { dard } \\
\text { units) }\end{array}$ & $\begin{array}{l}\text { Tom- } \\
\text { por- } \\
\text { ature } \\
\left.\text { ( }{ }^{\circ} \mathrm{C}\right)\end{array}$ & Wall number \\
\hline \multicolumn{10}{|c|}{ Western Three Forks Valley } \\
\hline+.95 & 12 & .6 & $\mathbf{S}$ & - & $05-21-91$ & 1,420 & 7.3 & 10.0 & 02N02W26ACCA01 \\
\hline-- & 100 & -- & $\mathbf{S}$ & $\mathbf{D}$ & $05-21-91$ & 928 & 7.8 & 10.0 & 02N01W22ADDD01 \\
\hline 6.37 & 20 & -- & $\mathbf{D}$ & $\mathbf{D}$ & $05-21-91$ & 1,300 & 7.1 & 10.0 & 02N01W22DABA01 \\
\hline 10.85 & 1,200 & 16 & $\mathbf{D}$ & D & -- & -- & -- & - & 02N01E04BCAA01 \\
\hline 14.05 & 360 & 10 & D & D & -- & -- & -- & - & 02N01 E04BDCC 01 \\
\hline 12.36 & 450 & 12 & $\mathbf{D}$ & $\mathbf{D}$ & - & -- & - & - & 02N0IE04DBDC01 \\
\hline 11.94 & 280 & 7.6 & D & $\mathbf{D}$ & -- & -- & - & - & 02N01E04DBDD01 \\
\hline 42.97 & 1,200 & 5.9 & D & $\mathrm{D}$ & -- & -- & -- & - & 02N01E17ADBC01 \\
\hline 30.00 & 260 & 3.6 & $\mathrm{D}$ & $\mathbf{D}$ & $05-23-91$ & 851 & 7.5 & 10.5 & 02N01E25CBBD01 \\
\hline 12.05 & $<1$ & -- & - & $\mathbf{D}$ & $05-23-91$ & 755 & 7.3 & 9.5 & 02N01E26DDDA01 \\
\hline 68.01 & 12 & .1 & D & $\mathbf{D}$ & $05-22-91$ & 1,340 & 7.8 & 11.0 & 02N01E28DBDC01 \\
\hline 71.91 & 3 & - & $\mathrm{D}$ & $\mathbf{D}$ & -- & -- & -- & $\cdots$ & 02N01E28DCAB01 \\
\hline 13.73 & 60 & 30 & D & $\mathbf{D}$ & $05-22-91$ & 460 & 7.4 & 8.0 & 02N01E28DCBC01 \\
\hline-- & -- & -- & $\mathbf{S}$ & $D$ & -- & -- & -- & - & 02N01E35DDDA01 \\
\hline 22.78 & 200 & 1.2 & $\mathbf{R}$ & $\mathbf{D}$ & - & -- & - & - & 02N01E36BAAC01 \\
\hline 8.46 & 20 & .3 & D & $\mathbf{D}$ & $09-24-92$ & 469 & 7.8 & 8.5 & 02N02E20DDCB01 \\
\hline 3.19 & -- & - & - & - & -- & -- & -- & $-\infty$ & 02N02E28CADCO1 \\
\hline 3.35 & -- & $\cdots$ & - & - & -- & -- & -- & - & 02N02E28CADCO2 \\
\hline 3.80 & - & -- & - & - & - & -- & -- & - & 02N02E28CADC03 \\
\hline 3.32 & 99 & -- & $\mathbf{D}$ & $\mathbf{D}$ & $06-25-91$ & 602 & 7.7 & 9.5 & 02N02E28CADC04 \\
\hline$* 18$ & $\cdots$ & -- & - & $\mathbf{D}$ & - & - & $\ldots$ & - & 02N02E30DCBC01 \\
\hline 26.28 & 12 & .1 & $D$ & $\mathbf{D}$ & $05-23-91$ & 1,590 & 8.1 & 11.0 & 01N01 W13BCCC01 \\
\hline 8.73 & 15 & .4 & D & D & $05-23-91$ & 922 & 8.3 & 10.0 & $01 N 01$ WI3BDDD01 \\
\hline 5.00 & 20 & 3.3 & D & $\mathrm{D}$ & $05-23-91$ & 880 & 7.3 & 9.5 & 01N0I W27AAAB01 \\
\hline 78.00 & 25 & .4 & $\mathbf{D}$ & D & $05-24-91$ & 1,010 & 7.0 & 12.0 & 01N0I W27BBAAOI \\
\hline 71.87 & 30 & .8 & D & D & -- & -- & -- & -- & 01N0I W27BBAA02 \\
\hline 15.60 & 45 & -- & D & D & $05-31-91$ & 510 & 7.7 & 16.0 & 01N01 W31BDDA01 \\
\hline 19.86 & 25 & 1.0 & $\mathbf{D}$ & $\mathbf{D}$ & $05-29-91$ & 562 & 7.8 & 10.5 & 01N01E19DDCB01 \\
\hline 301.77 & 10 & .3 & D & D & $05-29-91$ & 1,470 & 7.2 & 13.0 & 01N01E29AAAA01 \\
\hline 83.29 & 25 & 2.5 & $\mathrm{D}$ & D & $05-29-91$ & 782 & 7.8 & 10.0 & 01N02E06BCBA01 \\
\hline 56.42 & 26 & -- & D & D & -- & -- & - & - & 01N02E07AACA01 \\
\hline -- & -- & -- & - & - & $09-23-92$ & 1,180 & 7.3 & 11.0 & 01N02E 10DBAB01 \\
\hline 189.70 & 20 & -- & $\mathbf{S}$ & - & $05-30-91$ & 1,460 & 7.4 & 13.0 & 01N02E18DCCD01 \\
\hline$* 220$ & 30 & .8 & $\mathbf{D}$ & $\mathbf{D}$ & -- & - & - & - & 01N02E19CDDD01 \\
\hline 47.02 & 40 & 1.3 & $\mathbf{D}$ & $\mathbf{D}$ & $05-28-91$ & 736 & 7.6 & 12.5 & 01N02E22BADD01 \\
\hline
\end{tabular}


Table 1. Records of selected wells by basin, Northern Rocky Mountains intermontane basins, Montana (Continued)

\begin{tabular}{|c|c|c|c|c|c|c|c|c|c|}
\hline Woll number & $\begin{array}{c}\text { Altitude } \\
\text { of land } \\
\text { surface } \\
\text { (ft) }\end{array}$ & $\begin{array}{c}\text { Date well } \\
\text { constructed }\end{array}$ & $\begin{array}{c}\text { Geologlc } \\
\text { unlt }\end{array}$ & $\begin{array}{c}\text { Depth } \\
\text { of well } \\
\text { (ft) }\end{array}$ & $\begin{array}{l}\text { Diam- } \\
\text { oter } \\
\text { of } \\
\text { cesing } \\
\text { (In.) }\end{array}$ & $\begin{array}{l}\text { Type } \\
\text { of } \\
\text { finish }\end{array}$ & $\begin{array}{l}\text { Top of } \\
\text { open } \\
\text { Intor- } \\
\text { val } \\
\text { (ft) }\end{array}$ & $\begin{array}{l}\text { Primery } \\
\text { use of } \\
\text { water }\end{array}$ & $\begin{array}{c}\text { Date water } \\
\text { level } \\
\text { meseured }\end{array}$ \\
\hline \multicolumn{10}{|c|}{ Western Three Forks Valley-Continued } \\
\hline 01N02E22CDCC01 & 4,190 & $11-30-72$ & Ts & 90 & 6.0 & 0 & - & $\mathbf{H}$ & -- \\
\hline 01N02E30DCAC01 & 4,404 & $04-10-74$ & Ts & 320 & 6.0 & $\mathbf{P}$ & 300 & $\mathbf{U}$ & $05-30-91$ \\
\hline 01S01W34DCDD01 & 4,745 & $12-04-90$ & QTsu & 60 & 6.0 & $\mathbf{x}$ & 50 & $\mathbf{P}$ & $05-30-91$ \\
\hline 01S01E02BBAB01 & 4,521 & -- & Ts & 24 & 2.4 & 0 & - & $\mathbf{U}$ & $05-29-91$ \\
\hline 01S01E05BBCD01 & 4,281 & -- & Qal & 66 & 6.0 & - & -- & $\mathbf{H}$ & $05-29-91$ \\
\hline 01S01E21BBBB01 & 4,667 & $03-01-77$ & KAm & 467 & 6.0 & $\mathbf{P}$ & 425 & $\mathbf{s}$ & 03-01-77 \\
\hline 01S01E22DBAA01 & 4,715 & $03-01-60$ & Ts & 350 & 6.0 & 0 & - & $\mathbf{H}$ & $08-28-92$ \\
\hline 01S02E03DCCC02 & 4,332 & $06-20-74$ & Ts & 404 & 16.0 & $\mathbf{F}$ & 180 & I & $05-28-91$ \\
\hline 01S02E10ABBB01 & 4,317 & 09-14-78 & Ts & 450 & 16.0 & G & 79 & 1 & $05-15-91$ \\
\hline 01S02E22BCBC01 & 4,339 & $11-11-66$ & Ts & 140 & 6.6 & $\mathbf{P}$ & 83 & $\mathbf{H}$ & $05-28-91$ \\
\hline 01S02E22CCDA01 & 4,480 & $07-02-78$ & Ts & 638 & 16.0 & $\mathbf{F}$ & 312 & $\mathbf{s}$ & $05-15-91$ \\
\hline 01S02E23BCBC01 & 4,595 & -- & Ts & -- & -- & - & -- & $\mathbf{U}$ & $05-15-91$ \\
\hline 02S02W01AAAA01 & 4,916 & $03-27-86$ & QTsu & 75 & 6.0 & $\mathbf{P}$ & 1 & I & $05-31-91$ \\
\hline 02S02W11BDBB01 & 5,048 & $12-02-73$ & KAm & 60 & 6.0 & $\mathbf{P}$ & 54 & $\mathbf{H}$ & $05-31-91$ \\
\hline 02S02W13ADBA01 & 5,023 & $08-29-78$ & QTsu & 107 & 6.0 & $\mathbf{P}$ & 100 & $\mathbf{H}$ & $06-08-91$ \\
\hline $02 \mathrm{~S} 02 \mathrm{~W} 18 \mathrm{DBCC} 01$ & 5,530 & -- & KAm & 141 & -- & - & - & $\mathbf{H}$ & $06-08-91$ \\
\hline 02S02W18DCAD01 & 5,450 & $05-11-78$ & Qal & 50 & 6.6 & $\mathbf{P}$ & 43 & H & $06-08-91$ \\
\hline 02S01E01AACB01 & 4,450 & $12-27-66$ & Ts & 137 & 6.0 & $\mathrm{x}$ & 129 & U & $06-07-91$ \\
\hline 02S01E02BCCB01 & 5,110 & $04-19-66$ & Ts & 630 & 6.0 & $\mathbf{P}$ & 586 & $\mathbf{S}$ & $04-19-66$ \\
\hline 02S02E05BDBB01 & 4,335 & $05-31-85$ & Qal & 60 & 6.0 & o & -- & H & $09-23-92$ \\
\hline 02S02E06BCCC01 & 4,385 & $12-14-65$ & QTsu & 74 & 6.0 & - & 74 & $\mathbf{s}$ & $06-07-91$ \\
\hline 02S02E18CDCD01 & 4,392 & $01-23-70$ & QTsu & 63 & 6.0 & o & -. & $\mathbf{U}$ & $06-07-91$ \\
\hline 02S03E19ACBC01 & 4,705 & $06-10-78$ & Ts & 45 & 6.0 & $\mathbf{P}$ & 25 & $\mathbf{H}$ & $05-28-91$ \\
\hline 02S03E19BDAB01 & 4,725 & 05-14-79 & Ts & 80 & 6.0 & $\mathbf{F}$ & 60 & $\mathbf{S}$ & $05-28-91$ \\
\hline 03S01W23ABBB01 & 4,865 & $07-07-87$ & QTsu & 70 & 6.6 & $\mathbf{P}$ & 60 & $\mathbf{H}$ & $06-07-91$ \\
\hline
\end{tabular}


Table 1. Records of selected wells by basin, Northern Rocky Mountains intermontane basins, Montana (Continued)

\begin{tabular}{|c|c|c|c|c|c|c|c|c|c|}
\hline $\begin{array}{l}\text { Water level } \\
\text { (ft) }\end{array}$ & $\begin{array}{l}\text { Dis- } \\
\text { charge } \\
\text { (gall } \\
\text { min) }\end{array}$ & $\begin{array}{c}\text { Specific } \\
\text { capacity } \\
\text { [(gal/ } \\
\text { min)/ft] }\end{array}$ & $\begin{array}{c}\text { Source } \\
\text { of dls- } \\
\text { charge } \\
\text { data }\end{array}$ & $\begin{array}{l}\text { Type } \\
\text { of log } \\
\text { avall- } \\
\text { able }\end{array}$ & $\begin{array}{c}\text { Date } \\
\text { water- } \\
\text { quality } \\
\text { parameler } \\
\text { measured }\end{array}$ & $\begin{array}{c}\text { Spectfic } \\
\text { conduc- } \\
\text { tance } \\
(\mu \mathrm{S} / \mathrm{cm})\end{array}$ & $\begin{array}{l}\text { pH } \\
\text { (stan- } \\
\text { dard } \\
\text { units) }\end{array}$ & $\begin{array}{l}\text { Tem- } \\
\text { per- } \\
\text { ature } \\
\left({ }^{\circ} \mathrm{C}\right)\end{array}$ & Well number \\
\hline \multicolumn{10}{|c|}{ Western Three Forks Valley-Continued } \\
\hline -- & 40 & 8.0 & D & D & $09-23-92$ & 628 & 7.5 & 13.5 & 01N02E22CDCCO1 \\
\hline 213.67 & 20 & .4 & D & D & - & -- & .. & .- & 01N02E30DCAC01 \\
\hline 11.24 & 22 & 7.3 & D & D & $05-30-91$ & 622 & 7.6 & 10.0 & 01S01W34DCDD01 \\
\hline 19.42 & -. & - & - & - & - & - & -- & -- & 01S01E02BBAB01 \\
\hline 31.05 & 8 & 1.2 & $\mathbf{S}$ & - & $05-29-91$ & 510 & 7.4 & 11.5 & 01S01E05BBCD01 \\
\hline$* 275$ & 15 & .6 & D & D & $05-30-91$ & 273 & 7.6 & 16.0 & 01S01E21BBBB01 \\
\hline 306.53 & -- & -- & - & D & -- & -- & -- & -- & 01S01E22DBAA01 \\
\hline 181.02 & 900 & 9.5 & D & D & $05-28-91$ & 1,490 & 7.4 & 16.5 & 01S02E03DCCC02 \\
\hline 62.33 & -- & -. & - & D & -- & $-\cdot$ & -- & -- & 01S02E10ABBB01 \\
\hline 68.02 & 20 & .3 & D & D & $05-28-91$ & 1,340 & 7.4 & 14.5 & 01S02E22BCBC01 \\
\hline 238.85 & -- & .. & - & $\mathbf{D}$ & -- & -. & -- & -- & 01S02E22CCDA01 \\
\hline 256.14 & .. & -- & - & - & -- & -. & -- & -- & 01S02E23BCBC01 \\
\hline 6.60 & 80 & 4.2 & D & D & $05-31-91$ & 280 & 7.2 & 8.0 & 02S02W01AAAA01 \\
\hline *9 & 30 & -- & D & D & $05-31-91$ & 297 & 6.6 & 8.5 & $02 \mathrm{~S} 02 \mathrm{~W} 11 \mathrm{BDBB} 01$ \\
\hline 62.04 & 10 & 5.0 & $\mathbf{S}$ & $\mathbf{D}$ & $06-08-91$ & 430 & 7.6 & 10.0 & 02S02W13ADBA01 \\
\hline 59.13 & 10 & .6 & $\mathbf{s}$ & - & $06-08-91$ & 377 & 7.2 & 11.0 & 02S02W $18 \mathrm{DBCC} 01$ \\
\hline 10.36 & 6 & 1.3 & $\mathbf{S}$ & D & $06-08-91$ & 266 & 7.2 & 8.5 & $02 S 02 W 18 D C A D 01$ \\
\hline 96.85 & 40 & 1.0 & D & D & $06-07-91$ & 336 & 7.5 & 17.5 & 02S01E01AACB01 \\
\hline$* 588$ & 10 & .2 & D & D & $06-07-91$ & 417 & 7.6 & 18.5 & 02S01E02BCCB01 \\
\hline 23.21 & 60 & 2.1 & $\mathbf{D}$ & $\mathbf{D}$ & $09-23-92$ & 362 & 7.7 & 13.0 & 02S02E05BDBB01 \\
\hline 64.37 & 15 & -- & D & D & -- & -- & -- & -- & 02S02E06BCCC01 \\
\hline 37.47 & 35 & 1.6 & $\mathbf{D}$ & $\mathbf{D}$ & $06-07-91$ & 398 & 7.7 & 10.5 & 02S02E18CDCD01 \\
\hline 20.08 & 7 & -- & $\mathbf{s}$ & D & $05-28-91$ & 989 & 7.8 & 8.5 & 02S03E19ACBC01 \\
\hline 40.26 & 9 & 1.4 & $\mathbf{S}$ & $\mathbf{D}$ & $05-28-91$ & 489 & 7.6 & 10.5 & 02S03E19BDAB01 \\
\hline 44.60 & 20 & 2.2 & D & D & $06-07-91$ & 747 & 7.3 & 11.5 & 03S01W23ABBB01 \\
\hline
\end{tabular}



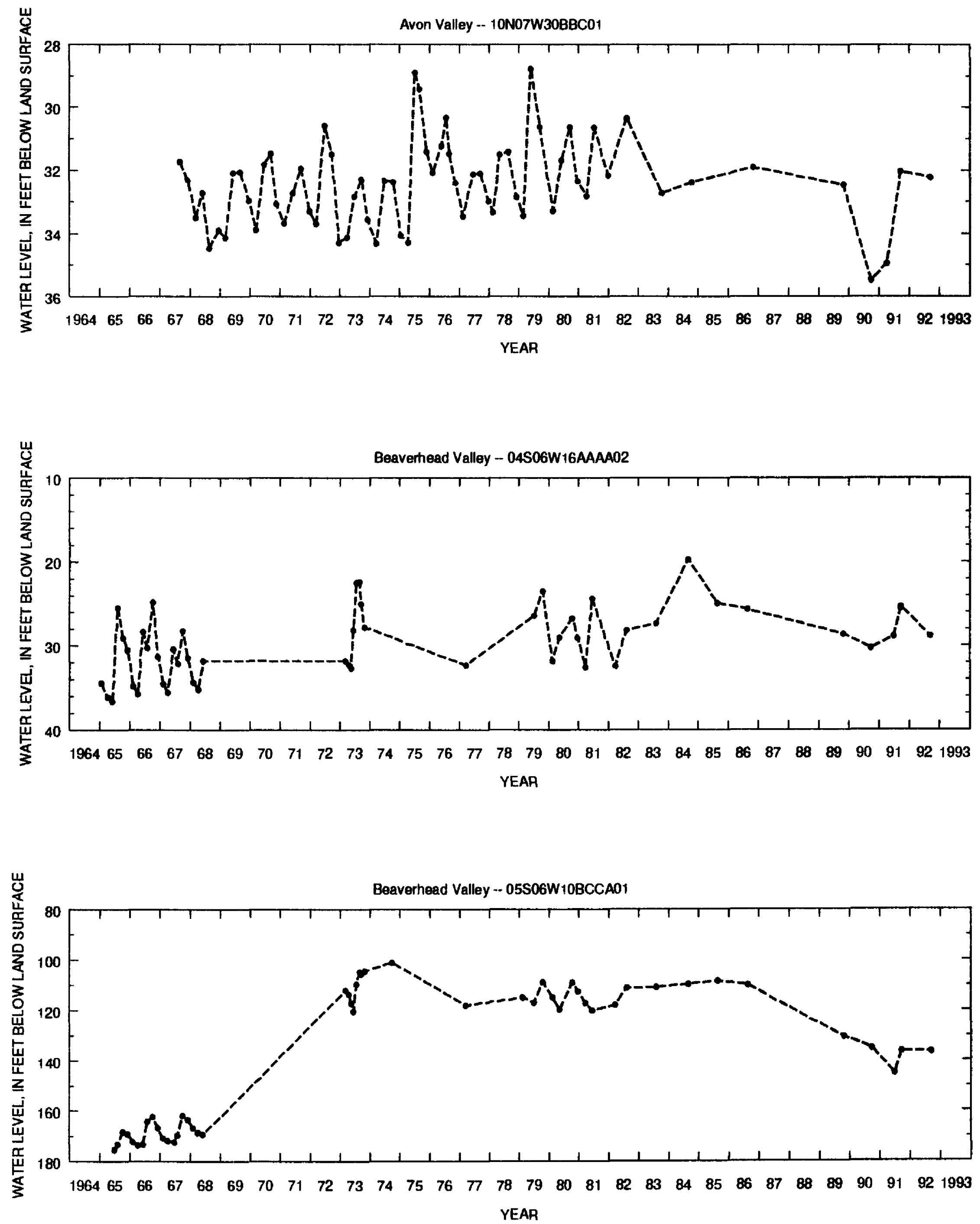

Figure 2. Hydrographs for selected wells, Northern Rocky Mountains intermontane basins, Montana. 

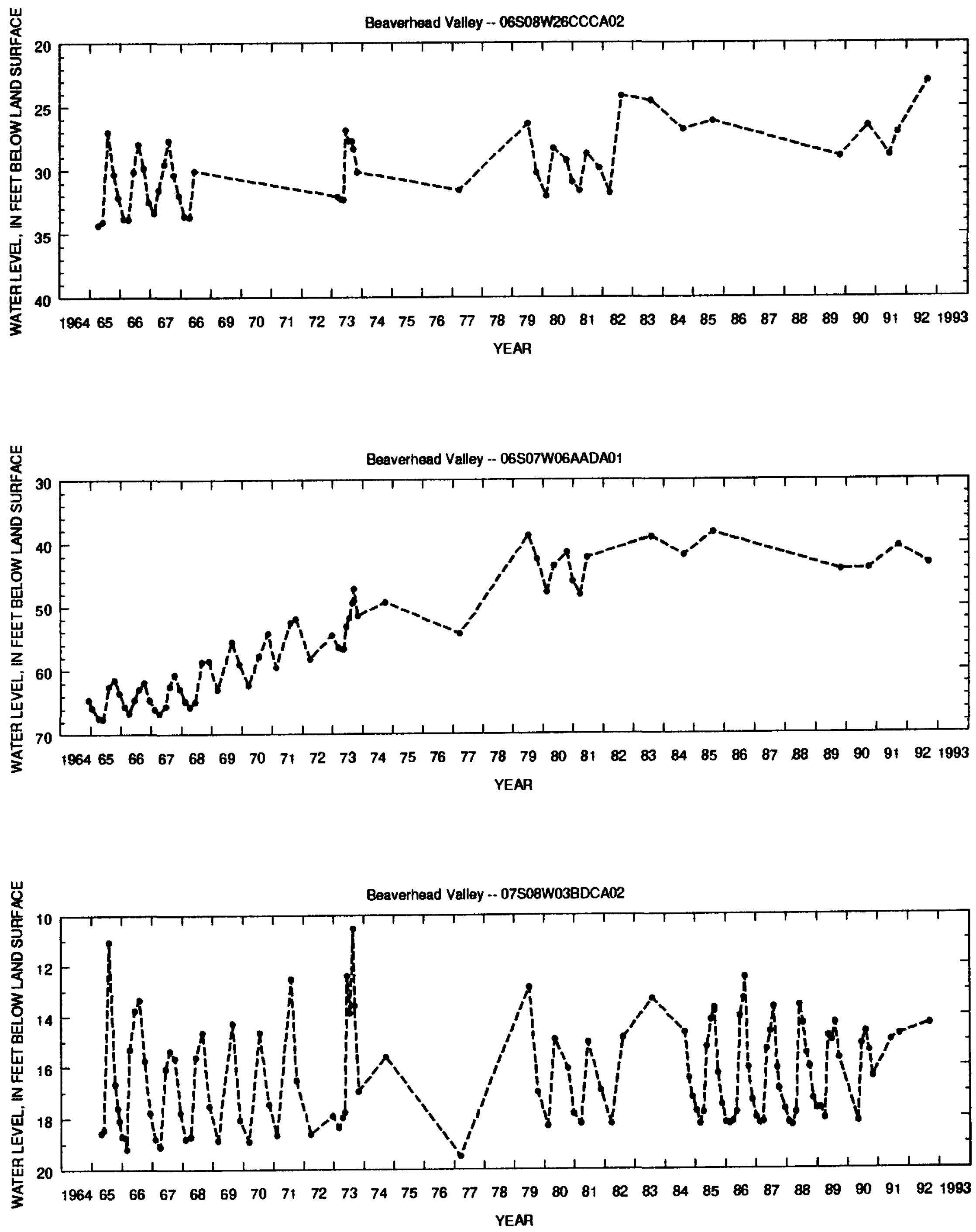

Figure 2. Hydrographs for selected wells, Northern Rocky Mountains intermontane basins, Montana (Continued). 

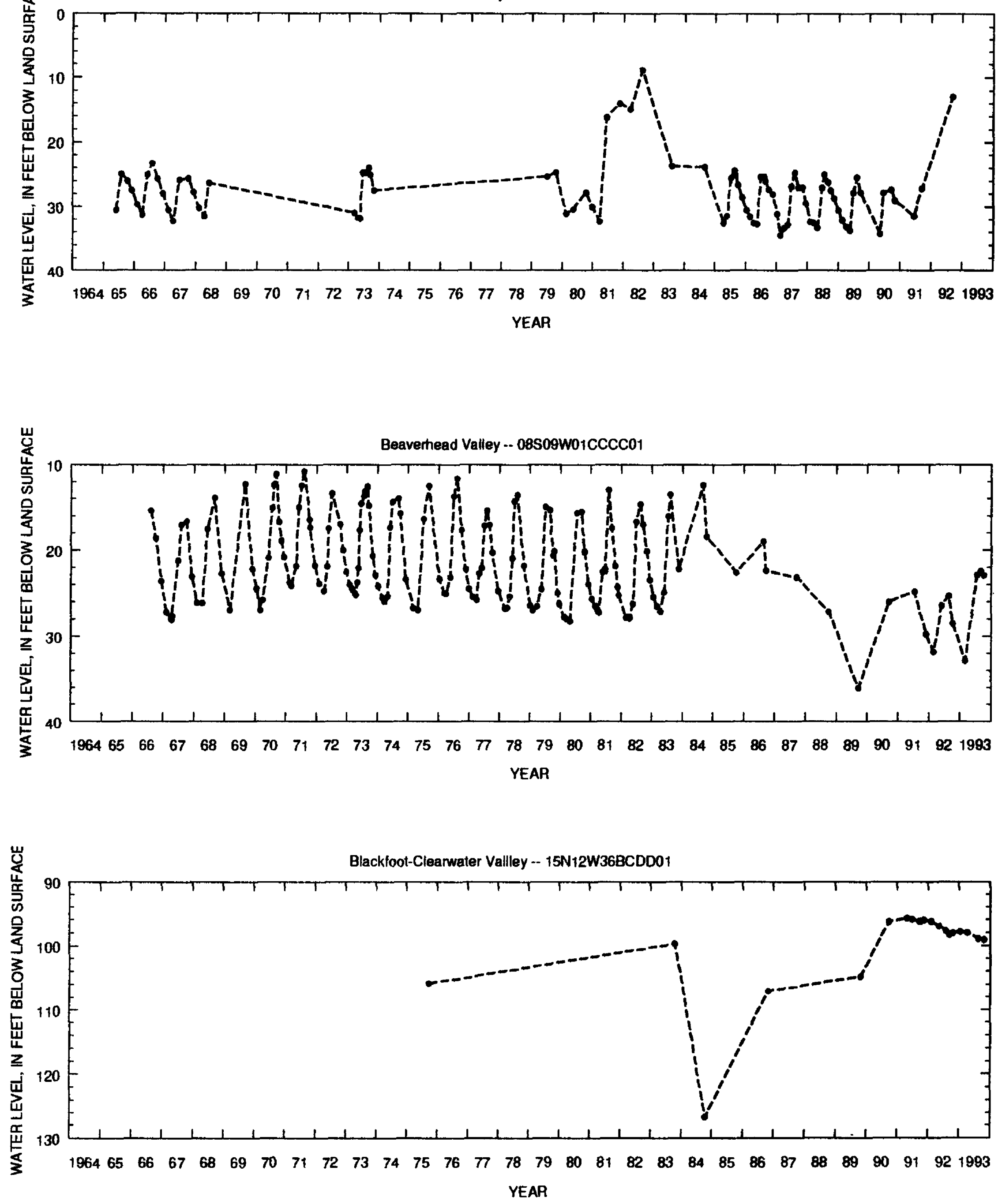

Figure 2. Hydrographs for selected wells, Northern Rocky Mountains intermontane basins, Montana (Continued). 

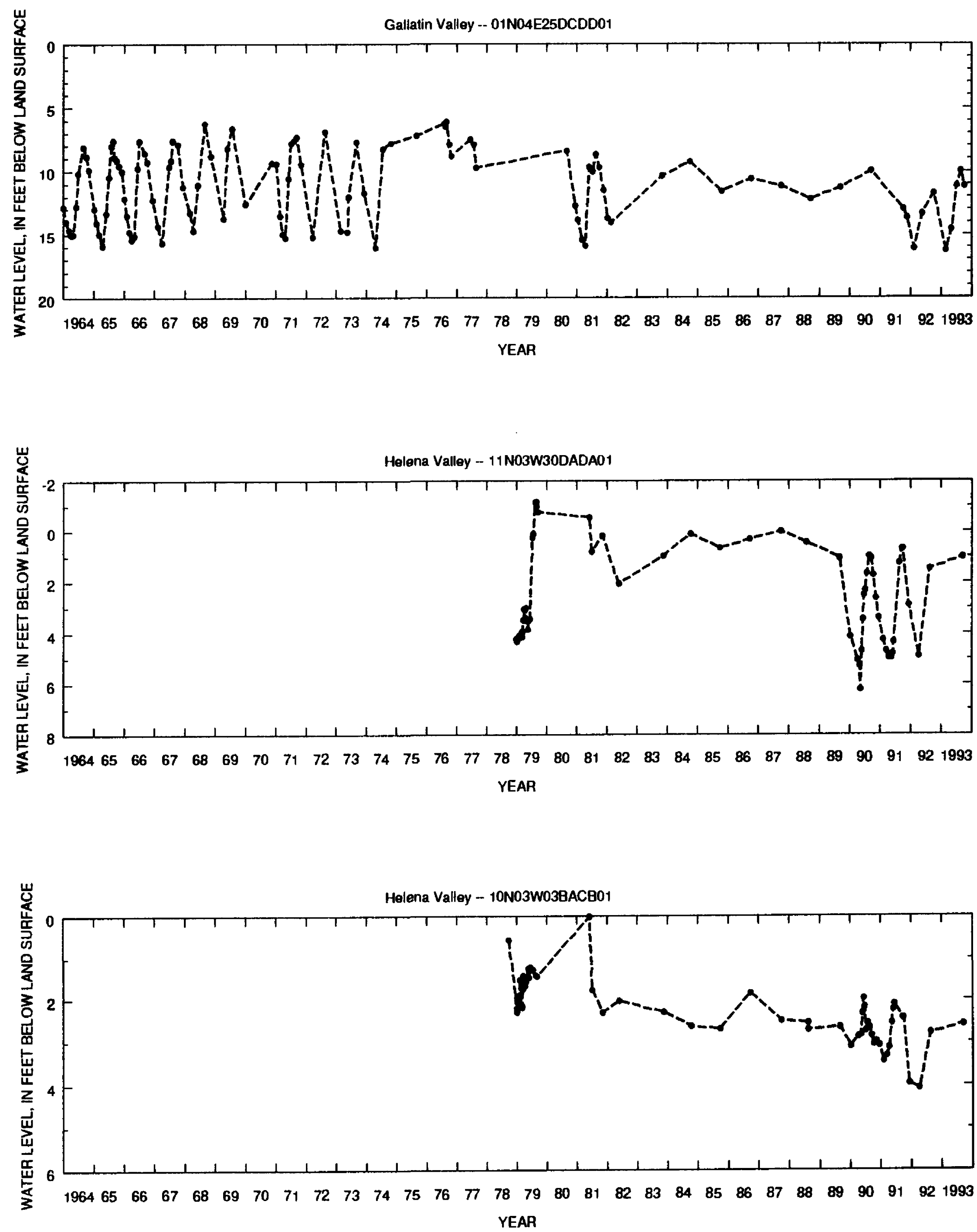

Figure 2. Hydrographs for selected wells, Northern Rocky Mountains intermontane basins, Montana (Continued). 

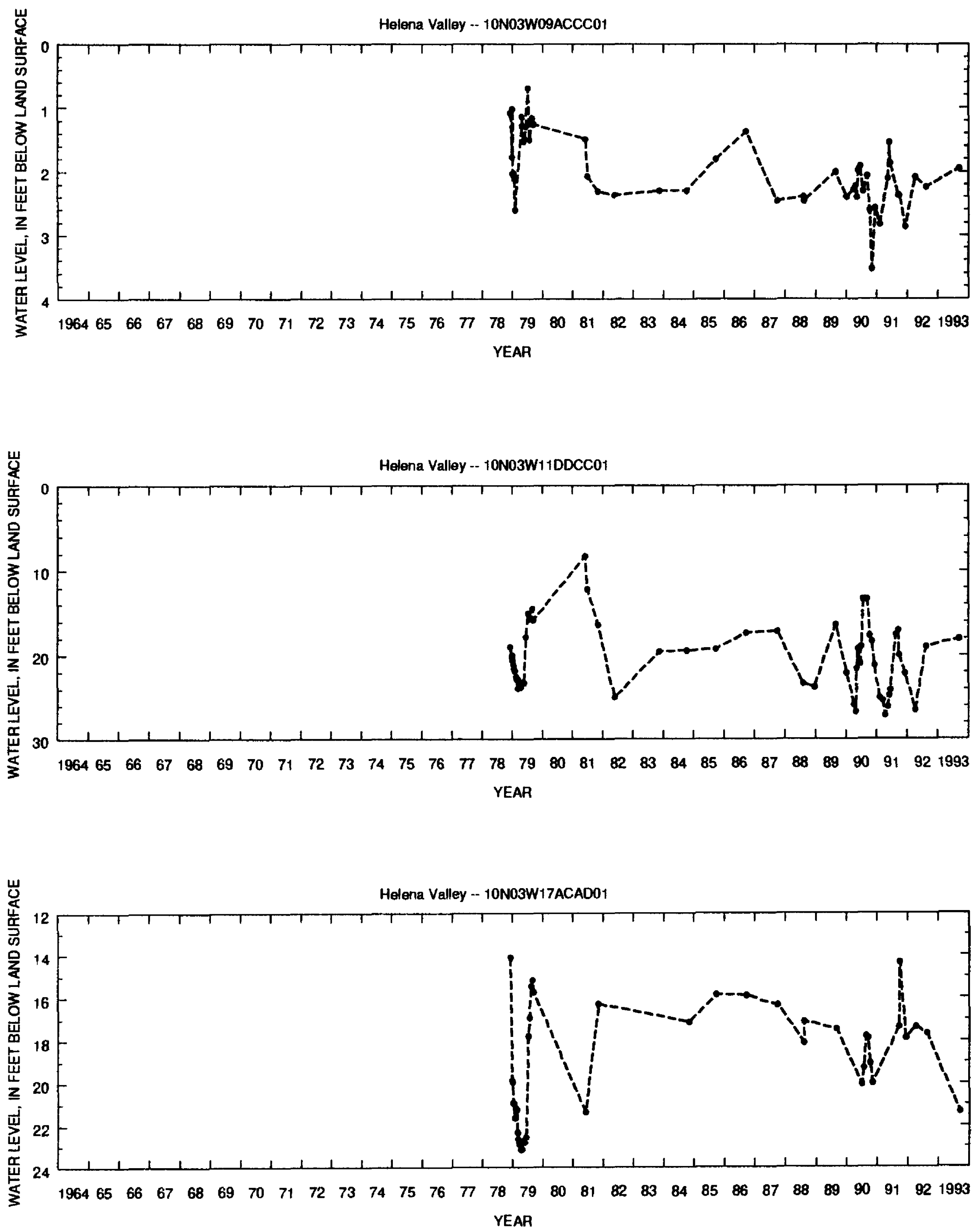

Figure 2. Hydrographs for selected wells, Northern Rocky Mountains intermontane basins, Montana (Continued). 

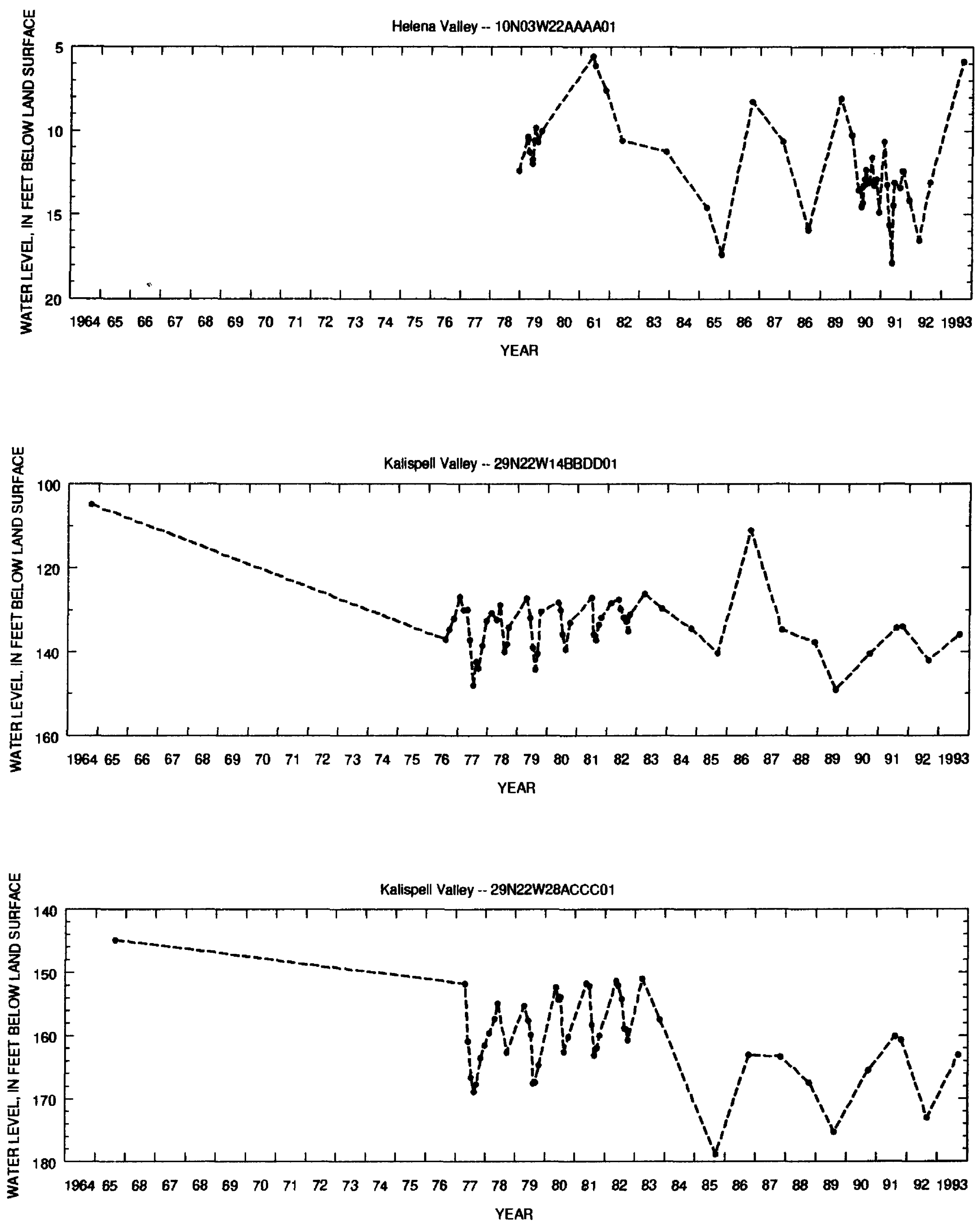

Figure 2. Hydrographs for selected wells, Northern Rocky Mountains intermontane basins, Montana (Continued). 

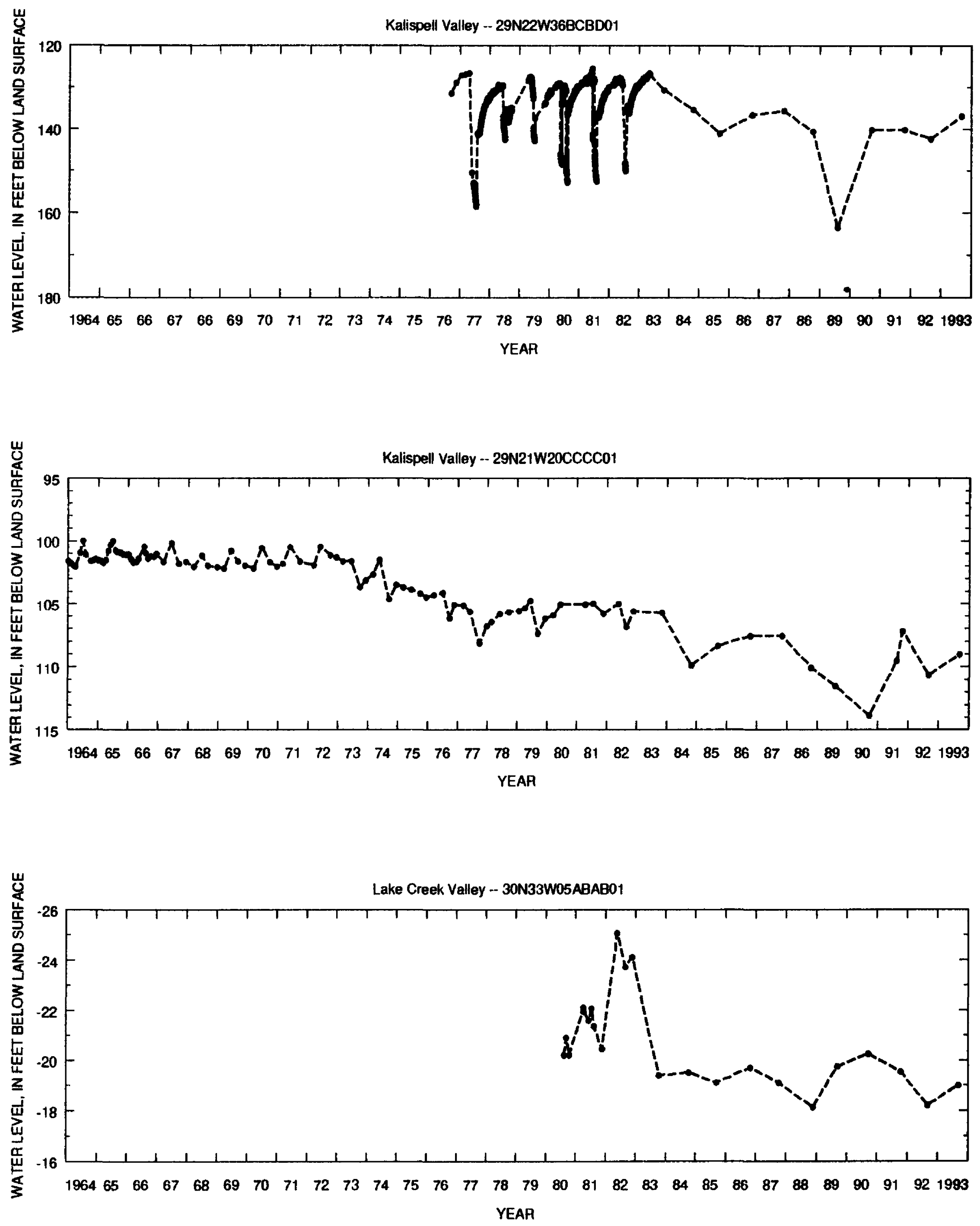

Figure 2. Hydrographs for selected wells, Northern Rocky Mountains intermontane basins, Montana (Continued). 

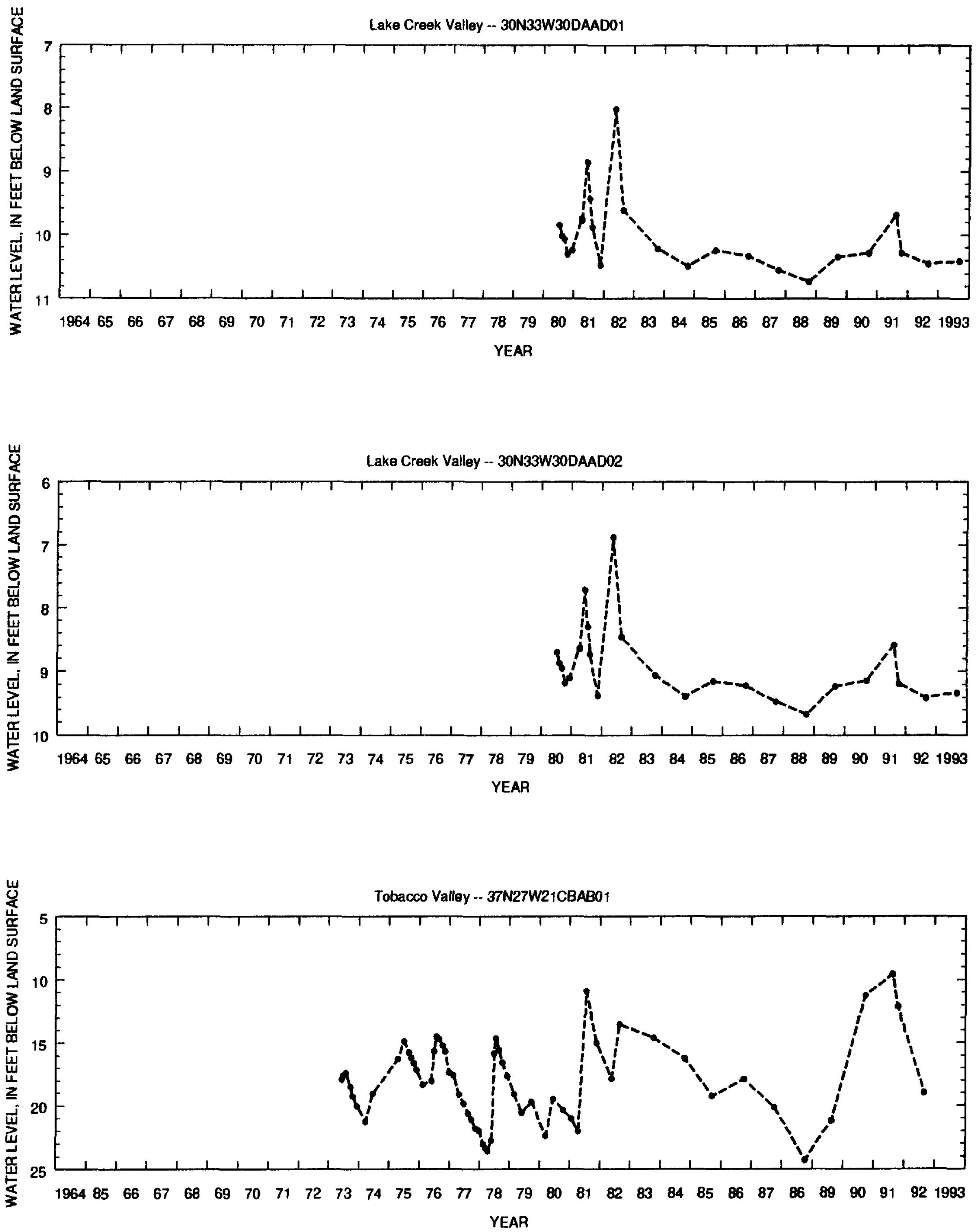

Figure 2. Hydrographs for selected wells, Northern Rocky Mountains intermontane basins, Montana (Continued). 

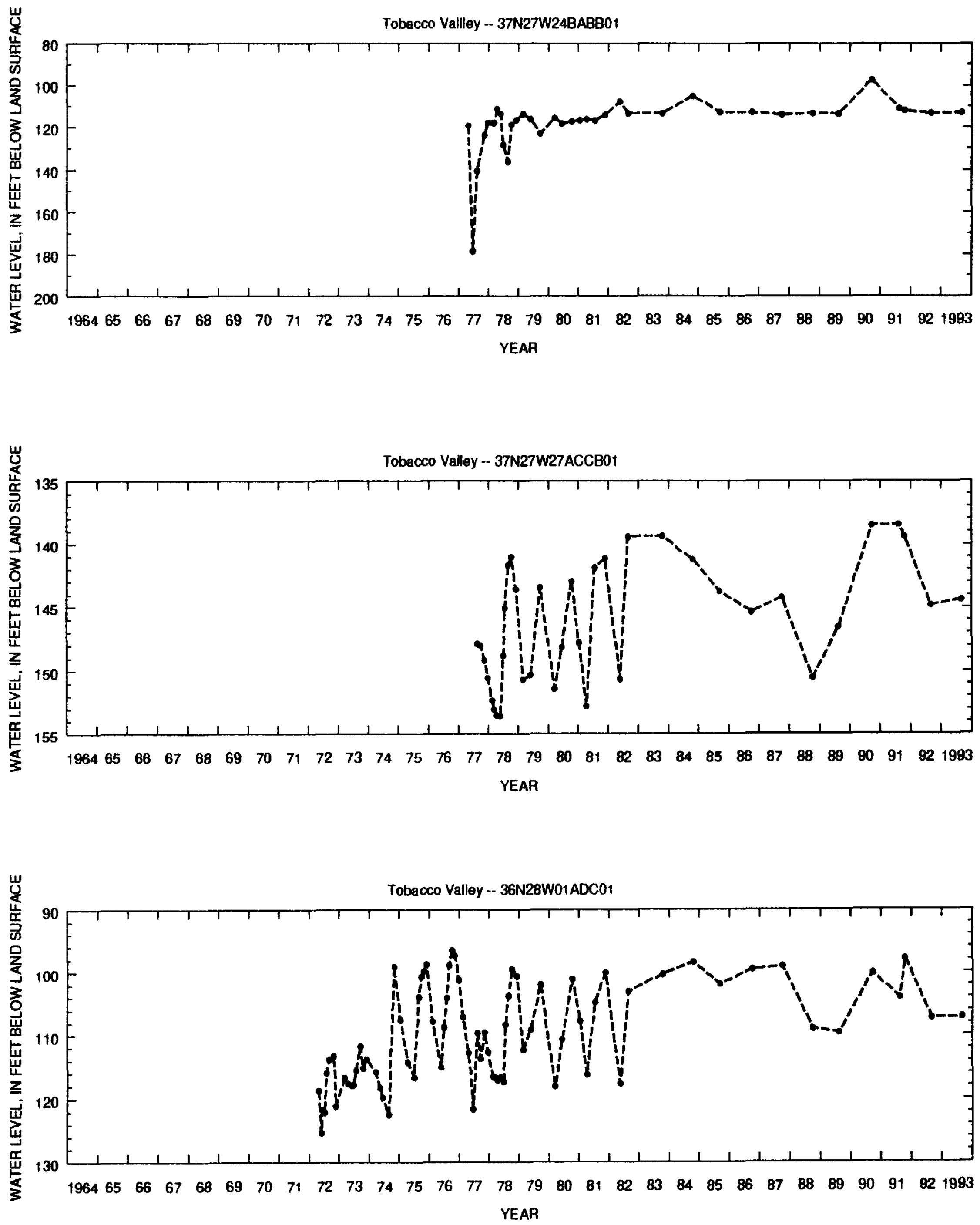

Figure 2. Hydrographs for selected wells, Northern Rocky Mountains intermontane basins, Montana (Continued). 

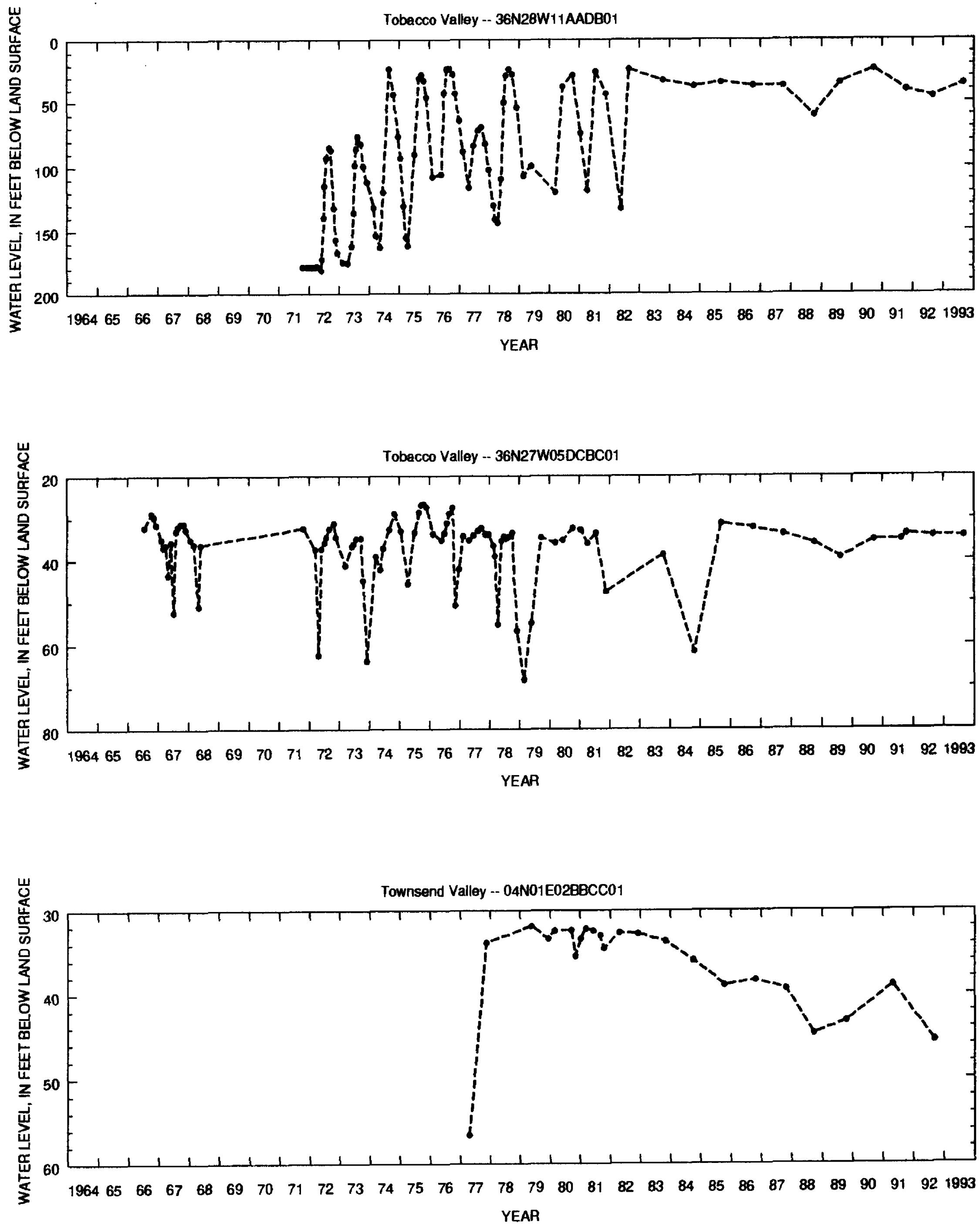

Figure 2. Hydrographs for selected wells, Northern Rocky Mountains intermontane basins, Montana (Continued). 

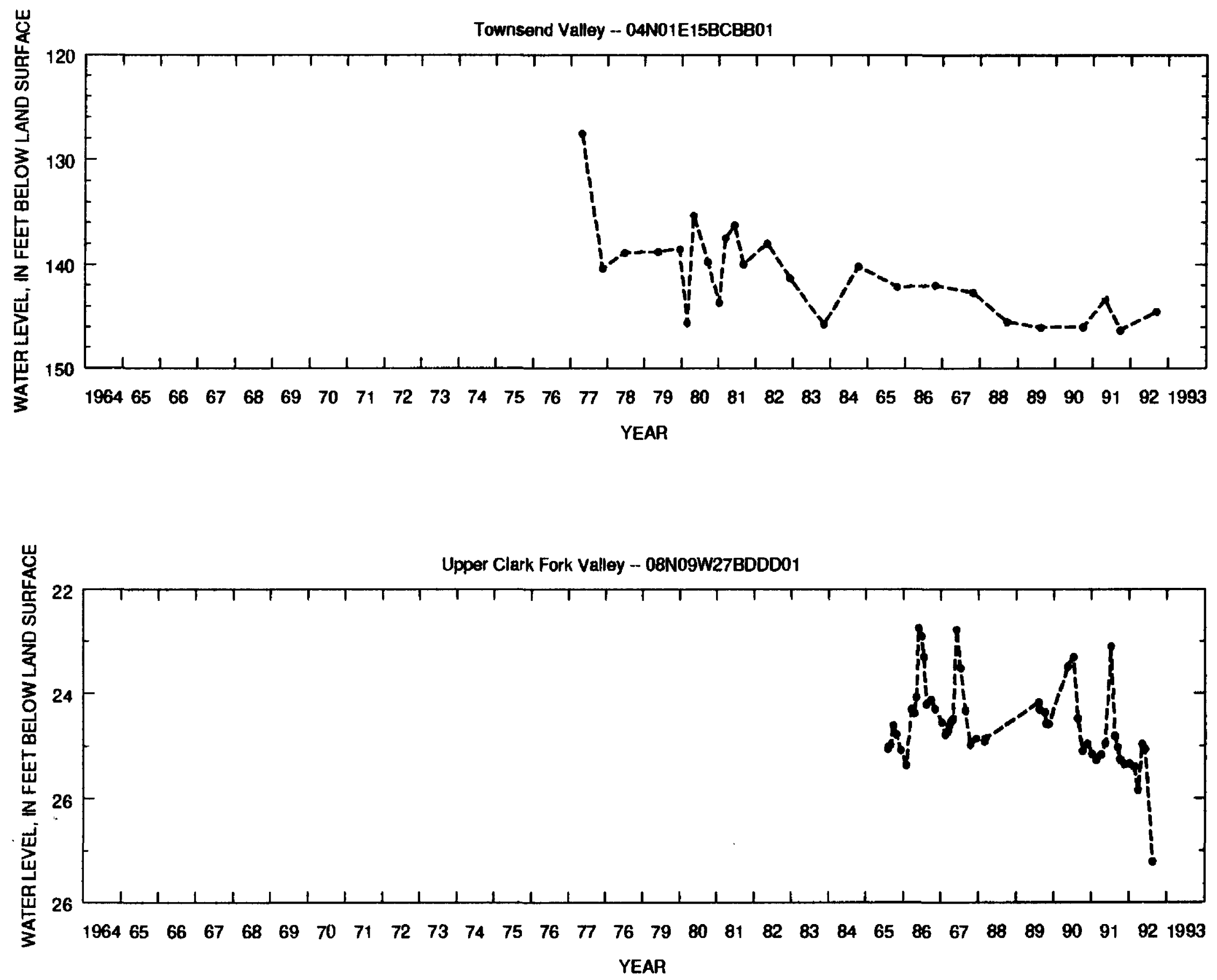

Figure 2. Hydrographs for selected wells, Northern Rocky Mountains intermontane basins, Montana (Continued). 